\title{
Investigation of the heterologous expression of the voltage activated potassium channel Kv1.7
}

\author{
Dissertation \\ zur Erlangung des Doktorgrades \\ der Mathematisch-Naturwissenschaftlichen Fakultäten \\ der Georg-August-Universität zu Göttingen
}

vorgelegt von

Rocio Karin Finol Urdaneta

aus Maracaibo/Venezuela

Göttingen 2004 
D7

Referent:

Prof. Dr. F.-W. Schürmann

Korreferent:

Prof. Dr. M. Hörner

Tag der mündlichen Prüfung: 29.04.04 
I. ABBREVIATIONS

1. INTRODUCTION

1.1 Cloning of Kv channels 1

1.2 Structure of Kv channels 3

1.3 Gating and kinetics of Kv channels 4

1.4. Physiological role of $\mathrm{Kv}$ channels 9

1.4.1 Hypoxia 9

$\begin{array}{ll}1.5 \text { Objective } & 10\end{array}$

2. MATERIALS AND METHODS 12

2.1 Molecular Biology Materials $\quad 12$

2.1.1 Equipment 12

2.1.2 Consumables 13

2.1.2.1 Kits 14

2.1.3 Chemicals 15

2.1.4 Oligonucleotides 16

2.1.5 Standards for DNA and RNA 16

2.1.6 Buffers and solutions $\quad 17$

2.1.7 Culture media 18

2.1.8 Plasmids 19

2.1.9 Enzymes and proteins $\quad 19$

2.1.10 Biological material 19

2.2 Cloning Methods 21

2.2.1 Tissue extraction 22

2.2.2 RNA extraction 22

2.2.3 Reverse transcription-polymerase chain reaction, RT-PCR 22

2.2.3.1 Reverse transcription "RT" 23

2.2.3.1.1 Standard protocol for Oligo(dT) $12-18$ or GSP 24

2.2.3.1.2 Standard protocol for random hexamers 24

2.2.3.1.3 RT protocol for GC rich targets 25

2.2.3.2 Polymerase chain reaction $\quad 26$

2.2.3.2.1 Taq polymerase $\quad 26$

2.2.3.2.2 Pfx polymerase $\quad 27$

2.2.3.2.3 BD advantage 2 polymerase 28

2.2.4 One step reverse transcription and PCR 28

2.2.5 Rapid amplification of cDNA ends "RACE" 29

2.2.6 Gel verification of the PCR products 30

2.2.7 Vector ligation 30 
2.2.8 Transformation 30

2.2.9 Bacterial culture 31

2.2.10 Plasmid isolation 31

2.2.11 Plasmid preparation 31

2.2.11.1 Restriction enzyme digestion 31

2.2.11.2 Fill in 32

2.2.11.3 Shrimp Alkaline Phosphatase "SAP" dephosphorylation 32

2.2.12 Sub-cloning into the oocyte expression vector pSGem 33

2.2.13 DNA sequencing 33

2.2.14 In-vitro transcription 33

2.2.15 Phenol/chloroform extraction $\quad 34$

2.2.16 Precipitation 34

2.2.17 RNA gel electrophoresis $\quad 35$

2.3 Cloning of kcna7 (mKv1.7) 35

2.3.1 Fragment F2-25 35

2.3.2 Fragment $82233 \quad 36$

2.3.3 Fragment EST 36

2.3.4 Amplification of kcna7 5' end 37

2.3.5 mKv1.7-T0 38

2.3.6 mKv1.7- $\Delta \mathrm{T} 1$

2.4 Cloning of KCNA7 (hKv1.7) 38

$\begin{array}{ll}2.5 \text { Promoter Analysis } & 40\end{array}$

$\begin{array}{ll}\text { 2.6 Protein Sequence Analysis } & 40\end{array}$

2.7 Electrophysiology Methods $\quad 41$

2.7.1 Oocyte extraction 41

2.7.2 Microinjection $\quad 42$

2.7.3 Two-electrode voltage clamp (TEVC) 43

2.7.4 Patch clamp 44

2.8 Instrumentation $\quad 45$

2.8.1 Mechanical set-up and electrical recordings 45

2.8.2 Micropipettes and electrodes 46

2.9 Stimulation Protocols and Data Analysis $\quad 47$

2.9.1 IV-protocol $\quad 47$

2.9.2 Tail protocol 48

2.9.3 Repetitive stimulation protocols 48

2.9.3.1 Continuous pulse protocol $\quad 48$

2.9.3.2 Cumulative inactivation protocol 48

2.9.4 Double pulse protocols $\quad 48$

2.9.4.1 Steady state inactivation $\quad 48$

2.9.4.2 Recovery from inactivation $\quad 49$

2.10 Leakage and Capacitive Current Correction 49

$\begin{array}{ll}2.11 \text { Data Analysis } & 50\end{array}$ 
2.11.2 Reverse potential $\quad 50$

2.11.3 Equilibrium potentials $\quad 50$

2.11.4 Calculation of the $\mathrm{IC}_{50} \quad 50$

2.11.5 Fits $\quad 50$

2.12 Statistics

3. RESULTS 53

3.1 Cloning of kcna7 53

$\begin{array}{ll}3.2 \text { Cloning of KCNA7 } & 60\end{array}$

$\begin{array}{ll}3.3 \text { Promoter analysis } & 63\end{array}$

3.4 Protein analysis $\quad 64$

3.5 Functional characterization of Kv1.7 channels 69

$\begin{array}{ll}3.5 .1 \mathrm{mKv} 1.7 & 69\end{array}$

$\begin{array}{ll}3.5 .1 .1 \text { lonic selectivity } & 73\end{array}$

$\begin{array}{ll}\text { 3.5.1.2 Influence of the extracellular ions } & 74\end{array}$

3.5.1.3 Activation of mKv1.7 channels $\quad 76$

3.5.1.4 Inactivation of mKv1.7 channels $\quad 79$

3.5.1.4.1 Effect of extracellular potassium on the inactivation 82

3.5.1.4.2 Steady state inactivation 84

3.5.1.4.2.1 Effect of extracellular potassium on the steady state inactivation 86

3.5.1.5 Cumulative inactivation of mKv1.7 channels 86

3.5.1.5.1 Influence of extracellular potassium on the cumulative inactivation 88

3.5.1.6. Recovery from inactivation of mKv1.7 channels 89

$\begin{array}{ll}3.5 .2 \mathrm{hKv} 1.7 & 91\end{array}$

3.5.2.1 Activation of hKv1.7 channels 93

3.5.2.2 Inactivation of hKv1.7 channels 94

3.5.2.3 Cumulative inactivation of hKv1.7 channels 95

3.5.2.4. Recovery from inactivation of hKv1.7 channels 95

3.6 Pharmacology of Kv1.7 channels 96

$\begin{array}{ll}3.6 .1 \text { Sensitivity to TEA } & 97\end{array}$

$\begin{array}{ll}\text { 3.6.2 Zinc } & 99\end{array}$

3.6.3 $\square$ M Conotoxin RIIIK 100

3.6.4 Conkunitzin-S 101

3.6.5 Redox sensitivity 102

4. DISCUSSION 105

4.1 Cloning of Kv1.7 Channels 105 
4.5 Functional Properties of Kv1.7 channels

4.6 Pharmacology of Kv1.7 Channels

4.6.2 Zinc block of Kv1.7 currents

4.7 Comparison of the results obtained in this work to the available data 


\section{ABBREVIATIONS}

\begin{tabular}{|c|c|}
\hline A & adenine \\
\hline$A$ & Ampere \\
\hline$\AA$ & Angstrom $\left(10^{-10} \mathrm{~m}\right)$ \\
\hline aa & amino acid \\
\hline bp & base pair \\
\hline BSA & bovine serum albumina \\
\hline${ }^{\circ} \mathrm{C}$ & degree Celsius \\
\hline C & cytosine \\
\hline $\mathrm{Ca}^{2+}$ & calcium ion \\
\hline$\left[\mathrm{Ca}^{2+}\right]_{\mathrm{cyt}}$ & cytosolic calcium concentration \\
\hline $\mathrm{CHO}$ & chinese hamster ovary \\
\hline $\mathrm{Cl}^{-}$ & chloride ion \\
\hline cDNA & complementary desoxyribonucleic acid \\
\hline cRNA & complementary RNA \\
\hline cos & green african monkey \\
\hline $\mathrm{C}-\mathrm{COOH}$-terminus & carboxy terminus of a protein \\
\hline DEPC & diethylpyrocarbonate \\
\hline DMSO & dimethylsulfoxide \\
\hline DNA & desoxyribonucleic acid \\
\hline DNase & desoxyribo nuclease \\
\hline dNTPs & desoxynucleoside triphosphate \\
\hline DTDP & dithiodipyridine \\
\hline DTT & dithiothreitol \\
\hline et al. & et alii \\
\hline \multirow[t]{2}{*}{ EGTA } & Ethylengycol-bis( $\square$-aminoethylether) \\
\hline & N,N,N',N'-Tetra acetic acid \\
\hline $\mathrm{EtOH}$ & ethanol \\
\hline G & guanine \\
\hline$\square G^{\circ}$ & Gibbs free energy \\
\hline G/V & conductance voltage relationship \\
\hline GSP & gene specific primer \\
\hline
\end{tabular}




\begin{tabular}{|c|c|}
\hline $\mathrm{h}$ & hour(s) \\
\hline HEK & human embryonic kidney \\
\hline Hepes & $\mathrm{N}-2-H y d r o x y e t h y l p i p e r a z i n e-\mathrm{N}$ '-2-ethane- \\
\hline & sulfonic acid \\
\hline $\mathrm{H}_{2} \mathrm{O}$ & water \\
\hline $\mathrm{Hz}$ & hertz \\
\hline 1 & inosine \\
\hline $\mathrm{IC}_{50}$ & half maximal inhibitory concentration \\
\hline$I_{K}$ & Potassium current \\
\hline$I_{\max }$ & maximal current \\
\hline IPTG & Isopropyl- b-D-thiogalactopyranoside \\
\hline $\mathrm{I}_{\mathrm{rem}}$ & remaining current \\
\hline IV & current voltage relationship \\
\hline $\mathrm{K}^{+}$ & potassium ion \\
\hline$\left[K^{+}\right]_{i}$ & intracellular $\mathrm{K}^{+}$concentration \\
\hline$\left[\mathrm{K}^{+}\right]_{\mathrm{o}}$ & extracellular $\mathrm{K}^{+}$concentration \\
\hline $\mathrm{K}_{\mathrm{v}}$ & voltage gated potassium channel \\
\hline $\mathrm{kb}$ & kilo base pair \\
\hline $\mathrm{kDa}$ & kilo dalton \\
\hline 1 & liter \\
\hline LB & Luria Broth \\
\hline$\square$ & micro $\left(10^{-6}\right)$ \\
\hline $\mathrm{m}$ & milli $\left(10^{-3}\right)$ \\
\hline M & molarity \\
\hline $\min$ & minute(s) \\
\hline mRNA & messenger RNA \\
\hline $\mathrm{n}$ & nano $\left(10^{-9}\right)$ \\
\hline $\mathrm{n}$ & number of independent experiments \\
\hline $\mathrm{Na}^{+}$ & sodium ion \\
\hline NFR & normal frog Ringer \\
\hline $\mathrm{nt}$ & nucleotide \\
\hline $\mathrm{N}$-, $\mathrm{NH}_{2}$-terminus & amino terminus of a protein \\
\hline OD & optic density \\
\hline ORF & open reading frame \\
\hline
\end{tabular}




\begin{tabular}{|c|c|}
\hline$\square$ & Ohm \\
\hline $\mathrm{p}$ & pico $\left(10^{-12}\right)$ \\
\hline \multirow[t]{2}{*}{$\mathrm{pH}$} & negative of the logarithm of the proton ion \\
\hline & concentration \\
\hline $\mathrm{P}_{\mathrm{o}} / \mathrm{P}_{\mathrm{o}} \max$ & relative open probability \\
\hline$P_{\text {open }}$ & open probability \\
\hline PAGE & polyacrylamide gel electrophoresis \\
\hline PCR & polymerase chain reaction \\
\hline Pfu & Pyrococcus furiosus \\
\hline RACE & rapid amplification of cDNA ends \\
\hline RBL & rat basofilic leukemic cell \\
\hline $\mathrm{RE}$ & endoplasmic reticulum \\
\hline RNA & riobonucleic acid \\
\hline RNase & ribonuclease \\
\hline RT-PCR & reverse trancriptase-PCR \\
\hline \multirow[t]{2}{*}{$\mathrm{RT}_{75}$} & time required to reach $75 \%$ of the maximal \\
\hline & current \\
\hline rpm & revolutions per minute \\
\hline sec & seconds \\
\hline SNPs & single nucleotide polymorfisms \\
\hline Taq & Thermus aquaticus \\
\hline $\operatorname{tau}_{\text {Inac }}$ & inactivation time constant \\
\hline $\operatorname{tau}_{\mathrm{Cl}}$ & cumulative inactivation time constant \\
\hline TEA & tetraethyl ammonium \\
\hline $\mathrm{TEA}_{\circ}$ & extracellular TEA \\
\hline TEA $_{i}$ & intracellular TEA \\
\hline TEVC & two-electrode voltage clamp \\
\hline TFs & transcription factor binding site sequences \\
\hline TRIS & tris(hydroxymethyl)aminomethane \\
\hline $\operatorname{tau}$ Inac & inactivation time constant \\
\hline$[\mathrm{T}]$ & toxin concentration \\
\hline V & potential in volts \\
\hline$V_{h}$ & holding potential \\
\hline
\end{tabular}


$\mathrm{V}_{1 / 2}$

wt

$X$. laevis

$X$-Ringer

$[\mathrm{X}]_{\mathrm{i}}[\mathrm{X}]_{\mathrm{o}}$

4-AP half activation potential

wild type

Xenopus laevis

Ringer solution with ion $\mathrm{X}$

intracellular and extracellular concentration of the ion of species $X$

4-aminopyridine 


\section{INTRODUCTION}

Ion channels are specialized membrane spanning proteins that permeate ions at a high rate when they are in the open conformation. Those proteins are known to contain an aqueous ion-selective pore region that creates an expedite pass through the plasma membrane. Each channel is specialized to conduct a particular set of ion species (cation or anion specific channels) or a specific single ion type to which is highly selective $\left(\mathrm{K}^{+}, \mathrm{Na}^{+}, \mathrm{Ca}^{2+}, \mathrm{Cl}^{-}\right)$. Ion channels use different mechanisms to open and close the pore (gating) in response to biological stimuli including binding of a ligand; mechanical deformation of the plasma membrane; and changes in the transmembrane potential or voltage (Hille, 2001; Armstrong \& Hille, 1998). The group of voltage activated ion channels includes a wide variety of members and the attempts to systematize its classification had focused in their ionic selectivity. Therefore, channels that are activated by voltage and selective for potassium ions are called $\mathrm{K}_{\mathrm{V}}$ channels; likewise those selective for sodium and calcium are $\mathrm{Nav}$ and Cav channels, respectively.

Ions tend to diffuse down their concentration gradient in order to equalize the concentration through solutions. Biological membranes are selectively permeable and offer a barrier to the free diffusion of ions generating an electrical potential difference (membrane potential $E_{m}$ ) established between the solution at both sides of the membrane. Voltage gated ion channels constitute very important proteins because $\mathrm{Kv}$ channel opening leads to an increase in the permeability for a certain ion that further generates the electrical signals in the cells. For instance, the opening of $\mathrm{Na} v$ channels induces a rapid increase of the membrane permeability for $\mathrm{Na}^{+}$, determining the rising phase of the action potential process. Cav channels have a major role in muscle contraction and in the activation of responses mediated by $\mathrm{Ca}^{2+}$ as second messenger that are crucial for nervous signaling. The voltage gated $\mathrm{K}^{+}$ channels are indispensable for the electrical excitability of nerve and muscle fibers because they are responsible of the repolarization of the cell membrane after the initiation of an action potential. Voltage gated $\mathrm{K}^{+}$channels are known to modulate synaptic transmission and secretion from endocrine cells, such as insulin from pancreatic islet cells (MacDonalds et al., 2001); dysfunction of Kv channels is associated to multiple pathophysiologycal conditions. Hence, $\mathrm{K}^{+}$channels have 
multiple and important functions in the signaling at the cellular level, thus much interest have been focused in the study of ion channels for many years. The cloning of the Shaker channel from the fruit fly Drosophila melanogaster (Tempel et al., 1987; Kamb et al., 1987; Pongs et al., 1988) represented a huge step in the study of Kv channels since it allowed the investigation of the proteins in isolation from other cellular events. Furthermore, once the different $\mathrm{Kv}$ channel genes were cloned, the recombinant proteins have been used for structural studies that include NMR and crystallographic determination of the tridimensional configuration of the ion channel proteins (Antz et al., 1997; Schott et al., 1998; Perozo et al., 1998; Morais-cabral et al., 1998; among others).

\subsection{Cloning of Kv channels}

The molecular cloning of ion channels has significantly contributed to the molecular understanding of the functioning of $\mathrm{K}^{+}$channels, thus several mammalian channels have been cloned and their biophysical properties characterized. The homology between the core "transmembrane" protein sequences of the $\square$-subunits, have served for the classification into the different subfamilies. Several cloning strategies had been used in the identification and study of ion channels. They involve Phage library screening, PCR, RT-PCR, protein-protein interaction cloning strategies, and in silico screening. Thus, a wide range of methods that have cleared up the way in the understanding of the structure and function of the ion channels, among other proteins.

In 1988 K. B. Mullis introduced the polymerase chain reaction (PCR), as a novel method of generating large amounts of a specific DNA fragment starting with very small amount of source DNA (Saiki et al., 1988). PCR represents an elegant reaction that doubles the quantity of a target DNA sequence with each repetition of a thermal cycle (amplification). The DNA polymerase from a heat resistant bacterium, Thermus aquaticus, is commonly used due to its stability, resistance to degradation at high temperatures, and fidelity in the synthesis of the product DNA. The "amplification" takes place by priming double-stranded (target) DNA with short single stranded DNA primers. The primers are complementary to the DNA target and 
anneal with the denatured target sequences for the polymerase to start the synthesis. The reaction can continue through numerous cycles of denaturation, annealing and synthesis until the nucleotide bases, primers or the enzyme are depleted. PCR has revolutionized modern biology and has widespread applications in the areas of forensics, diagnostics, and gene expression analysis.

In Drosophila four family members of voltage activated $\mathrm{K}^{+}$channels, designated Shaker, Shaw, Shal and Shab have been found to result from alternative splicing of a single gene (Kamb et al., 1988; Pongs et al., 1988; Schwarz et al., 1988). Each of them has particular biophysical characteristics that have served for the further classification of the Kv channel family in other species. Accordingly, mammalian homologues for all four Drosophila families (Kv1, Kv2, Kv3 and Kv4 respectively) have been cloned from individual genes and their biophysical properties characterized. Subsequent work has revealed a range of closely related genes that parallels the diversity of $\mathrm{K}^{+}$channels known in vivo, and to date over 80 different cDNAs corresponding to separate genes have been discovered.

\subsection{Structure of Kv channels}

All voltage dependent potassium channels are tetrameric assemblies of a basic $\square$-subunit (Isacoff et al., 1990; MacKinnon et al., 1991). The $\square$-subunits of Kv channels share a basic structural motif of six hydrophobic domains (S1-S6, Fig.1A), with a pore lining region, or "P-region", between transmembrane domains S5 and S6 that is responsible for the ion selectivity of the channel. S1 to S4 contain the "voltage sensor" and are responsible for the control of the open probability of the channel. The fourth hydrophobic segment, S4, contains a peculiar sequence of 5 to 8 positively charged amino acids (mainly arginine) at every third position. From experimental evidence the positively charged S4 have been involved in sensing the membrane potential difference (Sigworth, 1994; Bezanilla, 2000). The S4 and the P-region are the most highly conserved sequences among all voltage gated potassium channels. Recent crystallographic data obtained from the bacterial voltage gated potassium channel KvAP compared to the pore lining segments of the proton gated channel protein KcsA from the procaryote Streptomyces lividans revealed high similarities 
between both structures (Jiang et a., 2003; Doyle et al., 1998). The region that confers high selectivity to potassium, the selectivity filter, consists of a water filled multi-ion pore that has up to 7 ion binding sites spanning from the cavity to the extracellular mouth of the channel, where potassium ions are stabilized by a carbonyl oxygen backbone on the selectivity filter (Zhou et al., 2001). In 2003 an unexpected transmembrane pattern for the voltage sensor was obtained. In the bacterial KvAP channel the S4 helix, along with the second part of S3, forms an $\square$-helical hairpin that constitutes a paddle-like structure that extends out from the channel core into the membrane's fluid interior. The paddle is hydrophobic and it is not embedded in the protein core, but loose in the membrane with a flexible connection to the rest of the channel (Jiang et al., 2003).

The four $\square$-subunits of biologically relevant $\mathrm{K}^{+}$channels have been shown to constitute the pore of the channel. Homomultimeric channels are formed when identical $\square$-subunits are assembled together. Furthermore different $\square$-subunits belonging to the same subfamily can assemble and generate a heteromultimeric protein that usually has particular characteristics. Therefore, heteromeric Kv channels contribute to the big diversity of potassium currents identified in vivo materializing its molecular correlates (Ruppersberg et al., 1990; Wang et al., 1993). Additionally, accessory subunits ( $\square$-subunits) can associate to the tetrameric $\mathrm{K}^{+}$channels without participating from the pore structure but modulating the electrophysiological properties of them (Rettig et al., 1994).

\subsection{Gating and Kinetics of Kv channels}

The permeation through $\mathrm{K}^{+}$channels is regulated by the gating mechanism, which is important in determining the signaling behavior of the channel. For Kv channels, a positive change in the transmembrane voltage generates a conformational rearrangement that leads to the opening of the channel. As a consequence, the permeation of ions is evidenced by a fast rise in the current in a process called "activation" (Fig 2A). It has been suggested that $\mathrm{S4}$, physically linked to the outer helices, could open the pore by pulling the outer helices away from the central pore axis, causing the inner helices to follow (Jiang et al., 2003). 

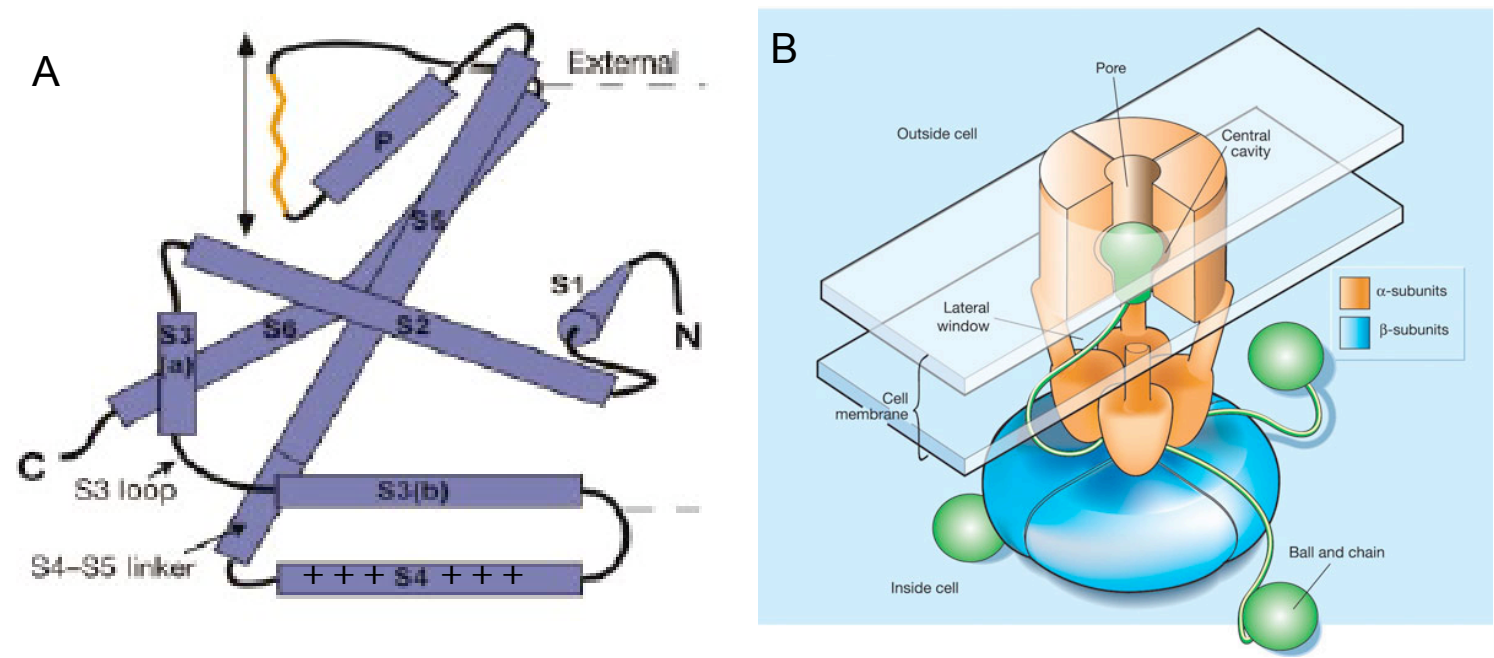

Figure 1. A, Schematic diagram of the KvAP subunit topology is shown with the selectivity filter and an arrow to indicate the ion pathway. The limit between the S4-S5 linker and S5 is indicated by a black line (from Jiang et al., 2003).

$\mathrm{B}$, Voltage-dependent potassium channel and the ball and chain model of channel inactivation. The channel is composed of four alpha-subunits (orange), which span the cell membrane (front subunit not shown), and four intracellular beta-subunits (blue). The $\square$-subunits have three components, the uppermost being the pore-forming domain. The "ball and chain" domains related to $\mathrm{N}$-inactivation are shown in green (from Aldrich, 2001).

Studies of Starace and Bezanilla propose a model similar to the one described for transporters where the outermost S4 position acts as a narrow "gate" separating the internal and external solutions (Starace \& Bezanilla, 2004). Nevertheless, at least other two mechanisms through which $S 4$ triggers pore opening by moving within, from inside to outside, the electric field have been proposed (Blaustein \& Miller, 2004).

The return of the membrane potential to the resting level generates a further conformational rearrangement that results in channel closing or deactivation. If after activation the depolarization is maintained, the channel will close despite the sustained activating stimulus, this type of closure is called inactivation (Fig $2 \mathrm{~A}$ ). $\mathrm{K}^{+}$ channels can display two modes of inactivation, fast and slow. Fast inactivation describes the rapid decay of currents in response to depolarization (millisecond range). It is due to the intracellular occlusion of the pore by a stretch of about 20 amino acids on the N-terminal region, known as the "ball" domain, tethered by "the chain" to S1 (Fig.1B, Fig.2A upper panel, and Fig.2B). Channels that inactivate 
A.

Activation

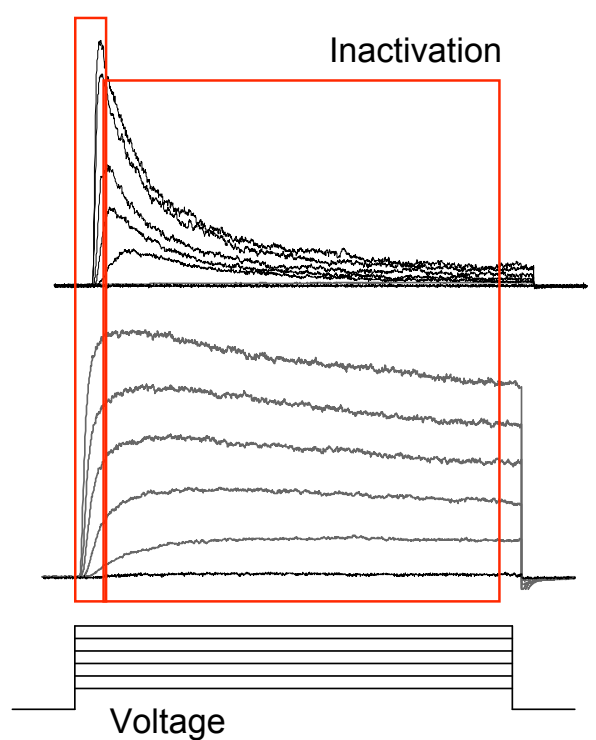

B.

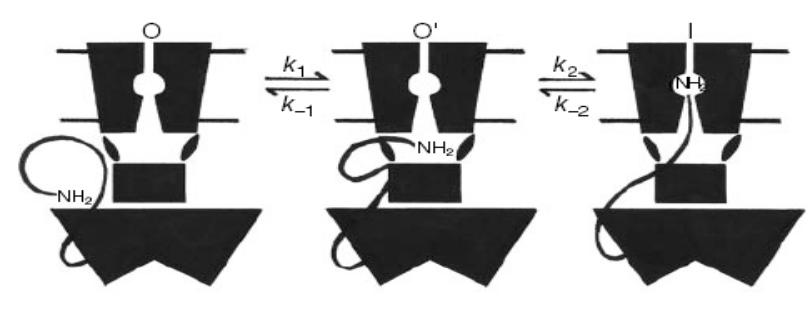

C.

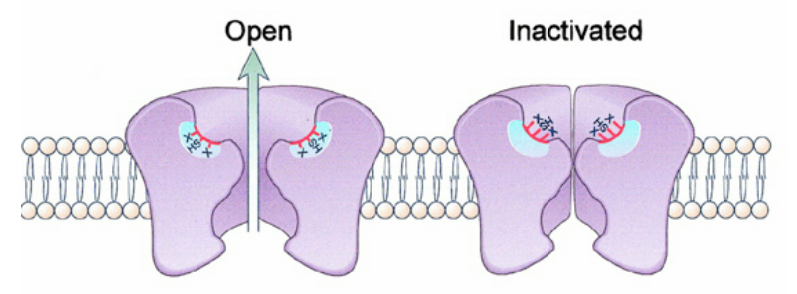

Figure 2. Operation of $\mathrm{Kv}$ channels. A. Activation and inactivation of $\mathrm{Kv}$ channels in response to membrane depolarization. Upper panel fast inactivating $\mathrm{Kv}$ channel. Lower panel slow inactivating $\mathrm{Kv}$ channel. $\mathrm{B}$. N-type inactivation, open $\mathrm{K}^{+}$channel with three different configurations of the $\mathrm{N}$-terminal inactivation gate shown attached to the $\mathrm{\square}$-subunit. For clarity, one inactivation gate is shown instead of four. $\mathrm{O}$, open channel with its $\mathrm{N}$-terminus before docking; O', open channel with its N-terminus bound to the hydrophilic protein surface; I, open channel with its $\mathrm{N}$-terminus entering the cavity (blocking the channel) (from Zhou et al., 2001).

$\mathrm{B}$, Slow C-type inactivation. Residues located near the external mouth of the channel pore control inactivation (closure of the channel) during depolarization. It involves cooperative effects of all $4 \mathrm{C}$-subunits and may involve region S6.

rapidly are recognized as "A-type". The first 20 amino acids on the $\mathrm{N}$-terminus of the प-subunit in Shaker-type channels constitute the inactivation gate that is located on the intracellular side of the membrane. The essential requirements that enable the $\mathrm{N}$-terminus to act as the "gate" include that the first approximately 10 amino acids should be predominantly hydrophobic (hydrophobic region) and the next 10 hydrophilic should contain an excess positive charge (hydrophilic region) (MurrelLagnado \& Aldrich, 1993). Several evidences support the idea that the inactivation gate binds to the pore. First, N-type inactivation can only occur after the voltagedependent gate opens, as if the opening of the pore would expose the "ball-receptor" to the gate (Zagotta \& Aldrich, 1990). Second, inactivation is produced by the binding of only one inactivation particle to the receptor within pore opening, even though each $\square$-subunit from homotetrameric $\mathrm{K}^{+}$channels has one inactivation particle $(4 \mathrm{x}-\mathrm{N}$ termini, Fig.1B) (MacKinnon et al., 1993; Gomez-Laguna \& Aldrich, 1995). Third, high concentrations of extracellular $\mathrm{K}^{+}$reduce inactivation, as if $\mathrm{K}^{+}$ions traversing the pore 
push the gate from its intracellular site (Demo \& Yellen, 1991). And fourth, the Ninactivation features resemble closely the action of intracellularly applied pore blockers like quaternary amines such as TEA (Armstrong, 1971). Furthermore, quaternary amines compete with the gate to inhibit $\mathrm{K}^{+}$current (Choi et al., 1991).

Slow inactivation was first observed on $\mathrm{N}$-terminal domain deletion mutant Shaker channels that had impaired fast inactivation. Slow inactivation occurs when channels are depolarized for seconds or minutes. It is also called core-associated or C-type inactivation, and is kinetically distinct from fast inactivation. The term "C-type inactivation" originated with the observation that Shaker splice variants with different carboxy-terminal regions (including S6) showed different rates of slow inactivation (Hoshi et al., 1991). C-type inactivation involves different structural elements that $\mathrm{N}$ type since it is not due to the direct block of the ion pathway by a inactivation particle, but it involves changes in the conformation of the channel outer vestibule (Yellen et al., 1998) (Fig. 2A lower panel, Fig 2C). In fact, C-type involves a reorientation of the residues in the pore region (Liu, 1996; Cha \& Bezanilla, 1997) that results in the interruption of the ion conduction by squeezing the pore shut along the P-region, closing the access between the deep pore and the extracellular solution (Yellen et al., 1994; Liu, 1996). This closure is believed to occur near a $\mathrm{K}^{+}$-selective binding site, because slow inactivation appears to require the prior evacuation of a $\mathrm{K}^{+}$ion from the pore to proceed ("foot in the door effect", Baukrowitz \& Yellen, 1996).

Furthermore, another type of inactivation has been described for Shaker channel mutants and Kv2.1 among other Kv channels. The term "P-type" inactivation was introduced by the group of De Biasi while describing the effect of a point mutation in the pore region (V369K) of Kv2.1 channels (De Biasi et al., 1993). This mutation resulted in a relatively fast inactivation of the ionic current but with different characteristics than $\mathrm{N}$ - and $\mathrm{C}$-type inactivation. P-type inactivation, like $\mathrm{N}$ - and $\mathrm{C}$ types of inactivation, is characterized by short bursts terminated by rapid entry into the inactivated state but unlike C-type, in which external tetraethyl ammonium (TEA) produces a simple blockade that slows inactivation and reduces currents, in P-type inactivated channels external TEA and $\mathrm{K}^{+}$generate an increase in the current, by enhancing channel availability and recovery from inactivation (De Biasi et al., 1993). 
According to Loots and Isakoff (1998), P-type inactivation corresponds to a first conformational rearrangement of the $\mathrm{Kv}$ channel protein that closes the permeation pathway, and only after that C-type inactivation can proceed via a second rearrangement that shifts the voltage dependence of the gating charge movement and yields a stabilized closed conformation. Thus, both forms of inactivation involve a single external gate that is closed in P-type; and the further rearrangement of S4 stabilizes the closed state. In turn, the N-type inactivation can trap channels in the activated state by two means: directly by preventing deactivation for as long as the $\mathrm{N}$ terminal ball occupies the internal mouth of the channel as shown by Bezanilla. or indirectly by accelerating the entry into the C-type inactivated conformation (Bezanilla et al., 1991). The former process most likely stabilizes the activated state of an "extruded" conformation of S4 that could explained the time dependence but voltage independence of the onset of inactivation, as well as the voltage dependence of the recovery from inactivation. Finally, the finding that channels undergo P-type inactivation at voltages of low open probability indicates that it is the transmembrane disposition of S4, rather than the state of the activation gate, what determines the conformation of the external inactivation gate (Baukrowitz \& Yellen, 1995; Loots \& Isacoff, 1998).

After inactivation channels must recover in order to readily activate again, a process recognized as "recovery from inactivation". The time course of the recovery from inactivation depends on the type of inactivation that preceeded. It has been described that during $\mathrm{N}$-type inactivation the inactivation particle, "the ball", gets trapped in the cavity of the $\mathrm{Kv}$ channel, therefore the intracellular gate must reopen to release it. This phenomenon is equivalent to the open channel block described for TEA. The results of whole-cell patch clamp recordings have shown that there is a linear increase in recovery rate with increasing $\left[\mathrm{K}^{+}\right]_{0}$, hense extracellular potassium modulates the recovery from C-type inactivation (De Biasi et al., 1993). There are indications that a low-affinity $\mathrm{K}^{+}$binding site involved in the recovery from inactivation must exist in the extracellular mouth of the channel. Furthermore, recovery from inactivation is voltage dependent with an increase in the rate of recovery upon hyperpolarization. The present model of a mechanistic interpretation of this process involves a $\mathrm{K}^{+}$ion bound to the extracellular part of the permeation pathway that destabilizes the inactivated state and increases the rate of recovery from $\mathrm{C}$-type 
inactivation. The modulation of C-type inactivation thereby provides a mechanism for autoregulation of $\mathrm{K}^{+}$channel activity (Levy \& Deutsch, 1996a and b). The ability of $\mathrm{K}^{+}$ to regulate its own conductance can play an important role in the modulation of all physiological Kv functions.

\subsection{Physiological role of Kv channels}

The type of inactivation used for each channel to regulate the ionic responses is fundamental in the establishment of the electrical characteristics of the cells, i.e. neurons, myocites or secretory cells, since these kinetics determine the "switch" to several cellular processes. For example, $\mathrm{K}^{+}$currents $\left(\mathrm{I}_{\mathrm{K}^{+}}\right)$play critical roles in determining cardiac electrical activities. Besides stabilizing resting potential, $I_{K_{+}}$in cardiac cells also plays an important role in modulating cellular excitability and regulating membrane repolarization. Therefore, $\mathrm{I}_{\mathrm{K}_{+}}$is an important determinant of action potential initiation. An important cardiac $\mathrm{K}^{+}$current is the transient outward $\mathrm{K}^{+}$ current $\left(I_{\text {to }}\right)$, which is known to be critical for initiating cardiac repolarization in the early phase of action potentials. In the absence of precise molecular correlates, currents are defined by its kinetic properties and pharmacological profile. Hence, $I_{t o}$ is a fast transient outward $\mathrm{K}^{+}$current that is rapidly activated and inactivated, and blocked by millimolar concentrations of 4-aminopyridine (4-AP) although the criterion of 4-AP sensitivity is not exclusive (Carmeliet, 1999).

\subsubsection{Hypoxia}

A number of studies have determined the properties, of channels formed by recombinant $\mathrm{Kv}$ channel subunits expressed in heterologous systems. For example, expression of Kv2.1 or coexpression of Kv2.1 with Kv9.3 (electrically silent $\square$-subunit) in COS cells causes hyperpolarization (Patel et al., 1997). Hypoxia decreases the $I_{k+}$ generated by either Kv2.1 or Kv2.1/Kv9.3 channels transfected in COS or mouse L cells. Because the $\mathrm{Kv}$ channels contain phosphorylation sites, these effects on $I_{K+}$ may be related to phosphorylation of the channel protein. Hypoxia is known to significantly decrease $\mathrm{I}_{\mathrm{K}_{+}}$in COS cells transiently transfected with $\mathrm{Kv} 3.1 \mathrm{~b}$ channels 
(Osipenko et al., 2000); PC12 cells or Xenopus oocytes transfected with Kv1.2 (Conforti et al., 2000); and HEK293 cells cotransfected with Kv4.2 and Kv1.2 (PerezGarcia et al., 1999). The common pathways in the transduction of hypoxic signals from the sensor to appropriate gene expression involve phosphorylation and/or redox modification of responsive transcription factors that include HIF-1 and p53 among many others that upon binding to the promoter enhance or suppress a number of hypoxia responsive genes (Bunn \& Poyton, 1996). Thus, promoters can be seen as the central processing unit of gene transcription, which by definition comprises the 5'end of the transcribed sequence.

\subsection{Objective}

$\mathrm{Kv} 1.7$ channels are the last cloned members of the Kv1 family of voltage activated potassium channels. From previous work it is known that the murine and human Kv1.7 channels are encoded by the kcna7/KCNA7 genes located at chromosomes 7 and 19, respectively. Transcripts of the kcna7 gene are reported present in several tissues including strong expression in the heart and skeletal muscle (Kalman et al., 1998). Likewise, Northern blots on human tissue revealed that the highest expression of KCNA7 is in skeletal muscle and heart (Kashuba et al., 2001). However, the literature about the biophysical and pharmacological characteristics of the murine Kv1.7 channels is scarce and controversial while there is no information available on the functional properties of the ortholog channel from human.

The objective of the present study was the cloning and functional characterization of the murine and human orthologs of the Kv1.7 channels. For this purpose the following experimental approaches were pursued:

PCR and PCR related techniques were used to clone the kcna7 and KCNA7 genes. Analysis of the DNA sequences was directed to the identification of important putative determinants of the regulation of these genes at the transcriptional and postranscriptional level. 
Functional characterization was done by means of expression of homomeric Kv1.7 channels on the Xenopus laevis oocyte heterologous system. The further biophysical characterization was performed by two electrode voltage clamp and patch clamp techniques.

Pharmacological characterization, including sensitivity to conotoxins was determined to establish functional mechanisms related to Kv1.7 channels in heterologous expression systems and more importantly in the physiology of voltage gated Kv1.7 channels in vivo.

The findings of the present work demonstrate the functional properties of the mammalian voltage activated $\mathrm{K}^{+}$channels $\mathrm{mKv1.7}$ and $\mathrm{hKv} 1.7$ from skeletal and cardiac muscle from mouse and human. The observations of this study suggest that the homomeric Kv channels might be potential molecular components of relevant pathophysiological processes. 


\section{MATERIALS AND METHODS}

\subsection{Molecular Biology Materials}

\subsubsection{Equipment}

- Balances:

Sartorius analytical balance Master Pro LA 230A, Sartorius, Göttingen, Germany

Sartorius precision balance Master Pro LA 2200S, Sartorius, Göttingen, Germany

- Bio-photometer, Eppendorf, Köln, Germany

- Centrifuges and mixers:

MiniShaker MS2, Migge Laborbedarf, Heidelberg, Germany

Thermomixer Eppendorf Comfort, Eppendorf, Köln, Germany

Microfuge: Roth, Karlsruhe, Germany

Centrifuge 5415D, Eppendorf, Köln, Germany

Centrifuge 5804R, Eppendorf, Köln, Germany

Superfuge RC5B Sorvall-Kendro, Hamburg, Germany

- Concentrator Speed-Vac 5301, Eppendorf, Köln, Germany

- Gel documentation system: Image Master TM VDS, Pharmacia Biotech/Fuji film thermal imagine system, Amersham-Pharmacia, Freiburg, Germany

- Incubators and water baths:

Microbiological Incubator B6120, Kendro, Hamburg, Germany

Shaking Incubator GFL 3032, Merck, Hannover, Germany

Bath-thermostat with integral bath DC 10-P5, Roth, Karlsruhe, Germany

Water bath thermostat Hecht Assistant, Schütt, Göttingen, Germany

- LaminAir HBB 2448 Laminar flow chamber, Heraeus-Kendro, Hamburg, Germany 
- Osmometer: VAPRO 5520, Wescor Inc., Logan, USA.

- Pipettes and pipettors:

Eppendorf Multipette Plus, Merck, Hannover, Germany

Eppendorf Reference 0.5-10 $\mu \mathrm{l}$, Merck, Hannover, Germany

10, 20, 200, $1000 \mu$ l Pipettes, Gilson, Bad Camberg, Germany

- Refrigerators and freezers:

Refrigerator KGT 3946 Premium, Liebherr Horst Plag, Schwalmstadt-Treysa, Germany

Ultra-freezer U570-85, New Brunswick Scientific, Nürtingen, Germany

- Thermocycler T3, Biometra, Göttingen, Germany

- Transilluminator CHROMA 52U, Laboratory instruments Vetter, Wiesloch, Germany

\subsubsection{Consumables}

Disposable syringes, 2, 5, 10, $30 \mathrm{ml}$

13 and $50 \mathrm{ml}$ Falcon tubes, BD Labware, Heidelberg, Germany

Glass capillaries:

Injection 3,5“ Drummond, Drummond, USA

TEVC Borosilicate Filament, Hilgenberg, Germany

Patch Clamp Kimax-51, Kimble products, USA

Pasteur pipettes short size (150 mm), WU Mainz, Germany

Pipette tips: 10, 20,100, and $1000 \mu$, Molecular Bioproducts, San Diego, USA Disposable Petri dishes:

$35 \times 10$ Nunclon $^{T M}$. Nalgene Nunc International, Denmark

30x15 Falcon, BD Labware, Heidelberg, Germany

35x10 Falcon, BD Labware, Heidelberg, Germany 
Reaction tubes

$0.5 \mathrm{ml}$ PCR tubes, Biozym, Germany

$1.5 \mathrm{ml}$ Eppendorf tubes, Eppendorf, Germanz

$1.5 \mathrm{ml}$ sterile tubes, Biozym, Germany

RTV Silicon, GE Bayer Silicones, Germany

Thermal Super VDS film, Amersham Biosciences, Freiburg, Germany

\subsubsection{Kits}

- RNA isolation:

FastTrack® 2.0 mRNA Isolation Kit , Invitrogen, Karlsruhe, Germany

TRIzo|® LS Reagent, Invitrogen, Karlsruhe, Germany

RNAeasy mini Kit, Qiagen, Hilden, Germany

- DNA cleanup and isolation:

QIAquick PCR Purification Kit, Qiagen, Hilden, Germany

QIAEX II Gel Extraction Kit, Qiagen, Hilden, Germany

QIAquick Nucleotide Removal Kit, Qiagen, Hilden, Germany

Gel isolation: QiaEx II Extraction Kit, Qiagen Hilden, Germany

- Plasmid DNA isolation

Wizard ®Plus Minipreps DNA Purification System, Promega Mannheim, Germany

NucleoSpin, Macherey-Nagel, Dueren, Germany

Qiagen plasmid midi Kits, Qiagen, Hilden, Germany

- Others

First strand synthesis: SuperScript II Gibco BRL

Advantage cDNA PCR Kit, Clontech, BD Biosciences, Heidelberg, Germany

QuickChange Mutagenesis Kit, Stratagene, Amsterdam, The Netherlands

Superscript III OneStep RT-PCR, Invitrogen, Karlsruhe, Germany

RACE: Marathon cDNA Amplification Kit, BD Clontech, Heidelberg, Germany 


\subsubsection{Chemicals}

All chemicals were of analytical grade, and were purchased from Sigma-Aldrich, (Munich, Germany); and Merck (Darmstadt, Germany).

Ammonium chloride, Merck

Ampicilline, Roche

Barium chloride, Sigma

Calcium chloride, Merck

Calcium nitrate, Sigma

Collagenase, Worthington Biochemical Corporation

Diethylpyrocarbonate (DEPC), Sigma

Dimethylsulfoxide (DMSO), Sigma

dNTPmix, Promega

Dithiothreitol (DTT), Sigma

Dithiodipyridine (DTDP), Sigma

LE Seakem agarose, Biozym

Lithium chloride, Sigma

Magnesium sulfate, Merck

Penicilline-streptomicine (10000:10000), Gibco

Phenol (pH 7.49-7.79), Roth

Potassium chloride, Merck

Random hexamers, Boehringer

Rubidium chloride, Sigma

Sodium acetate, Sigma

Sodium chloride, Merck

Sodium dodecyl sulfate (SDS), Sigma

Tetracycline, Roche

Tetraethylammonium chloride, Sigma

Tris $\mathrm{HCl}$, Sigma

Zinc chloride, Sigma

3-(N-Morpholino)propanesulfonic acid, 4-Morpholinepropanesulfonic acid (MOPS), Sigma

4-(2-Hydroxyethyl)piperazine-1-ethanesulfonic acid (HEPES), Sigma 
Isopropyl- b-D-thiogalactopyranoside (IPTG), Roche

5-bromo-4-chloro-3-indolyl-beta-D-galactopyranoside (X-Gal), Roche

Zinacef: Sodium cefuroxim, Hoesch

\subsubsection{Oligonucleotides}

All oligonucleotides (primers) were purchased from Metabion $\mathrm{GmbH}$, PlaneggMartinsried, Germany.

\begin{tabular}{cl}
\hline Primer & $5^{\prime}$ \\
\hline AP1 & CCA TCC TAA TAC GAC TCA CTA TAG GGC \\
P14 & TGG CAG TGT CTC GAG GCA AAA \\
P20 & TCC GAA TTC GTC ATC CTG GTC TCC A \\
P21 & ATT GGG CCC TCA CAC CTC AGT CAC CAT GTG \\
P23 & TCT GGC TGC TCT TCG AAT TTC CTG AGA \\
P27 & CCC TAG GAA TCT GTA CCC GCC ACC ATG \\
P38 & AGA ATG GAT CGT TGA AGG GCT GTC \\
P50 & ATT CGA AGA GCA GCC AGA GC \\
P99 & GCC ACA CGT CCG TTC ACC GGT C \\
P100 & GTG CGC GCC ATG GAG CCA CGG T \\
P106 & GCG GCC GCT CGG GCG GGA CCG CGCA GCC CTC GTC CTC CCG \\
& CAG CCG CGC CAG \\
P142 & ACC GCG CAG CCC TCG TCC TCC C \\
P186 & CCG GAC TTT CCA TCC TAT TTT TAC CCG \\
P222 & TCC CCG CTC CGC TGA ATG G \\
P223 & GTA GAC GGC GCT GGA AAA GAG GAC \\
P224 & CAC GTC GGT TCG CGG GT \\
P225 & CGG CCT GAG AGC TCT CGG GA \\
P270 & CTG GCA CGC CTG CGC GAG \\
P271 & GGG TGG ATT TCC AGG CAT TTG G \\
P272 & TTC CAG CGC CGT CTA CTT TGC CG \\
P273 & CTG CAG ACC TCA ACT GTT CCT CAC ACT TC \\
P289 & CTA CAA GGG AAA GCT CAA GAG ATC \\
\hline
\end{tabular}

\subsubsection{Standards for DNA and RNA}

Gene Ruler 100bp DNA Ladder, MBI Fermentas, St. Leon-Rot, Germany

Gene Ruler 1Kbp DNA Ladder, MBI Fermentas, St. Leon-Rot, Germany

RNA Ladder, low range, MBI Fermentas, St. Leon-Rot, Germany

RNA Ladder, high rage, MBI Fermentas, St. Leon-Rot, Germany 


\subsubsection{Buffers and solutions}

All solutions and buffers were prepared with bidistilled water.

\section{Oocyte solutions}

\begin{tabular}{|c|c|}
\hline \multicolumn{2}{|c|}{ Barth medium } \\
\hline $88 \mathrm{mM}$ & $\mathrm{NaCl}$ \\
\hline $1 \mathrm{mM}$ & $\mathrm{KCl}$ \\
\hline $7.5 \mathrm{mM}$ & Tris- $\mathrm{HCl}$ \\
\hline $2.4 \mathrm{mM}$ & $\mathrm{NaHCO}_{3}$ \\
\hline $0.82 \mathrm{mM}$ & $\mathrm{MgSO}_{4}$ \\
\hline $0.33 \mathrm{mM}$ & $\mathrm{Ca}\left(\mathrm{NO}_{3}\right)_{2}$ \\
\hline $0.41 \mathrm{mM}$ & $\mathrm{CaCl}_{2}$ \\
\hline osmolarity & $230-240$ mosmols \\
\hline
\end{tabular}

Ringer solutions

\begin{tabular}{cl}
\hline \multicolumn{2}{c}{ Normal Frog Ringer } \\
\multicolumn{2}{c}{ "NFR" } \\
\hline $115 \mathrm{mM}$ & $\mathrm{NaCl}$ \\
$2.5 \mathrm{mM}$ & $\mathrm{KCl}$ \\
$10 \mathrm{mM}$ & $\mathrm{Hepes}$ \\
$1.8 \mathrm{mM}$ & $\mathrm{CaCl}_{2}$ \\
\hline $\mathrm{pH} 7.2$ adjusted with $\mathrm{NaOH}$ \\
\hline
\end{tabular}

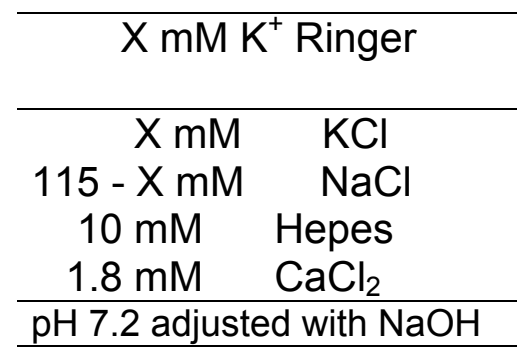

\begin{tabular}{|c|c|c|}
\hline \multicolumn{3}{|c|}{$\mathrm{X}^{+}$Ringer } \\
\hline 117.5 & & $\mathrm{XCl}$ \\
\hline 10 & $\mathrm{mM}$ & Hepes \\
\hline 1,8 & $\mathrm{mM}$ & $\mathrm{CaCl}_{2}$ \\
\hline \multicolumn{3}{|c|}{$\begin{array}{l}\text { pH } 7.2 \text { adjusted with } \\
\text { TrisOH }\end{array}$} \\
\hline
\end{tabular}

- Ampicilline solution: $20 \mathrm{mg} / \mathrm{ml}$ Ampicilline sodium salt in $\mathrm{H}_{2} \mathrm{O}$

- DEPC- $\mathrm{H}_{2} \mathrm{O} 0.1 \%$ DEPC in $\mathrm{H}_{2} \mathrm{O}$ : incubated overnight and autoclaved

- IPTG: $0.1 \mathrm{M}$ IPTG in $\mathrm{H}_{2} \mathrm{O}$

- X-Gal $30 \mathrm{mg} / \mathrm{ml} \mathrm{X-Gal} \mathrm{in} \mathrm{DMSO}$ 


\begin{tabular}{ll}
\hline \multicolumn{1}{c}{$5 \times$ TBE } & \multicolumn{1}{c}{ Loading buffer } \\
\hline $54 \mathrm{~g}$ Tris Base & $40 \%$ Glycerin \\
$27.5 \mathrm{~g}$ Boric Acid & $0.25 \%$ Bromphenol Blue \\
$20 \mathrm{ml} 0.5 \mathrm{M}$ EDTA & In $\mathrm{H}_{2} \mathrm{O}$ \\
\hline $\mathrm{pH} 8.0$ & \\
\hline
\end{tabular}

RNA electrophoresis buffers

\begin{tabular}{|c|c|}
\hline \multicolumn{2}{|c|}{ RNA running buffer } \\
\hline $1 x$ & MOPS \\
\hline $0.63 \mathrm{M}$ & Formaldehyde \\
\hline \multicolumn{2}{|c|}{ RNA sample buffer } \\
\hline $\begin{array}{l}750 \mu \mathrm{l} \\
150 \mu \mathrm{l} \\
240 \mu \mathrm{l} \\
400 \mathrm{mg} \\
500 \mathrm{\mu l} \\
5 \quad \mathrm{mg} \\
5 \mathrm{mg} \\
\text { RPA III }\end{array}$ & $\begin{array}{l}\text { Formamide (deionized) } \\
\text { 10x MOPS } \\
\text { Formaldehyde }(37 \%) \\
\text { Sucrose } \\
\text { Formamide } \\
\text { Bromphenol blue } \\
\text { Xylencyanol ff } \\
\text { (Ambion) }\end{array}$ \\
\hline
\end{tabular}

\begin{tabular}{ll}
\hline \multicolumn{3}{c}{ Sample elution buffer } \\
\hline $0,5 \mathrm{M}$ & $\mathrm{NH}_{4}$-Acetate \\
$1 \mathrm{mM}$ & EDTA \\
$0.2 \%$ & SDS \\
\hline
\end{tabular}

\begin{tabular}{cl}
\hline & \\
& Gel loading buffer \\
\hline $95 \%$ & Formamide \\
$0.025 \%$ & Xylencyanol \\
$0.025 \%$ & Bromphenol blue \\
$18 \quad \mathrm{mM}$ & EDTA \\
$0.025 \%$ & SDS \\
\hline
\end{tabular}

\subsubsection{Culture media}

\begin{tabular}{cc}
\hline LB Medium & Agar plates \\
\hline $10 \mathrm{~g}$ Trypton & $10 \mathrm{~g}$ Trypton \\
$5 \mathrm{~g} \mathrm{Yeast}$ & $5 \mathrm{~g} \mathrm{Yeast}$ \\
$5 \mathrm{~g} \mathrm{NaCl}$ & $5 \mathrm{~g} \mathrm{NaCl}$ \\
& $14 \mathrm{~g} \mathrm{Bacto-agarose}$ \\
$\mathrm{H}_{2} \mathrm{O}$ to $1000 \mathrm{ml}$ & $\mathrm{H}_{2} \mathrm{O}$ to $1000 \mathrm{ml}$ \\
\hline autoclaved & autoclaved \\
\hline Antibiotics were added when the mixture was at $50^{\circ} \mathrm{C}$.
\end{tabular}




\subsubsection{Plasmids}

p-Bluescript SK(+), Stratagene, Amsterdam, The Netherlands

pS-GEM: derived from pGEMHE vector (3022bp); Liman et al., Neuron, 1992, 9:86171). Kindly provided by Dr. Michael Hollmann, Bochum, Germany

pGem T-easy, Promega, Mannheim, Germany

\subsubsection{Enzymes and proteins}

Advantage 2 Polymerase Mix, BD Biosciences Clontech

DNA Polymerase I (Klenow) large fragment, Promega, Mannheim, Germany

Pfx-Polymerase, Life technologies

RNasin $40 \mathrm{U} / \mu \mathrm{l}$ Promega

Rnase $\mathrm{H}$, Invitrogen

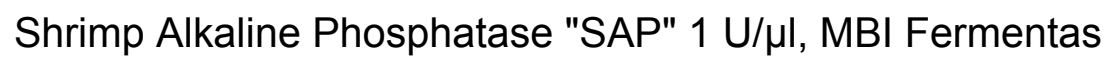

Superscript II and III RNase $\mathrm{H}$, Invitrogen

T4 DNA Ligase, New England Biolabs

T7 RNA Polymerase, Stratagene

Taq-Polymerase, Qiagen

Restriction enzymes/buffers were purchased from New England Biolabs, Amersham, MBI Fermentas, and Gibco.

\subsubsection{Biological material}

\section{RNA}

$\mathrm{BD}^{\mathrm{TM}}$ Premium Total mouse heart RNA (Clontech)

BD $^{\text {TM }}$ Premium Total human heart RNA (Clontech) 


\section{Conotoxins}

Conus peptides were kindly provided by Dr. Baldomero Olivera, Department of Biology, University of Utah, Salt Lake City, USA.

\section{Bacteria}

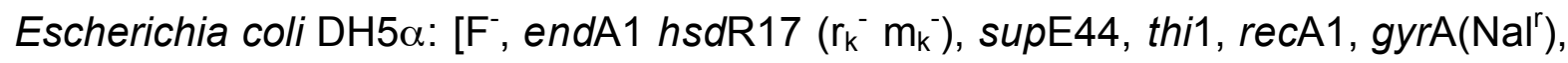
relA1, $\square($ lacZYA-argF)U169, $\square 80-d$ lacZ $\square \mathrm{M} 15]$.

Escherichia coli XL1-Blue MRF': $\square($ mcrA)183 $\square($ mcrCB-hsdSMR-mrr)173 endA1 supE44 thi-1 recA1 gyrA96 relA1 lac [F' proAB lacl ${ }^{q} \mathrm{Z} \square \mathrm{M} 15$ Tn10 $\left(\right.$ Tet $\left.\left.^{\mathrm{r}}\right)\right]^{\mathrm{c}}$

\section{Animals}

NMRL wild type Mus musculus specimens (tissues kindly provided by Mrs. Barbara Scheufler from the Department of Molecular Biology of Neuronal Signals (MaxPlanck- Institut für Experimentelle Medizin, Göttingen, Germany).

Xenopus laevis African frogs, Nasco, USA. 


\subsection{Cloning Methods}

All methods were performed following standard procedures according to Sambrook et al. 1989 (Sambrook et al., 1989). When commercial kits where used the protocol suggested by the manufacturer was carefully followed. The cloning strategy for most of the clones followed the sequence described in figure 3 .

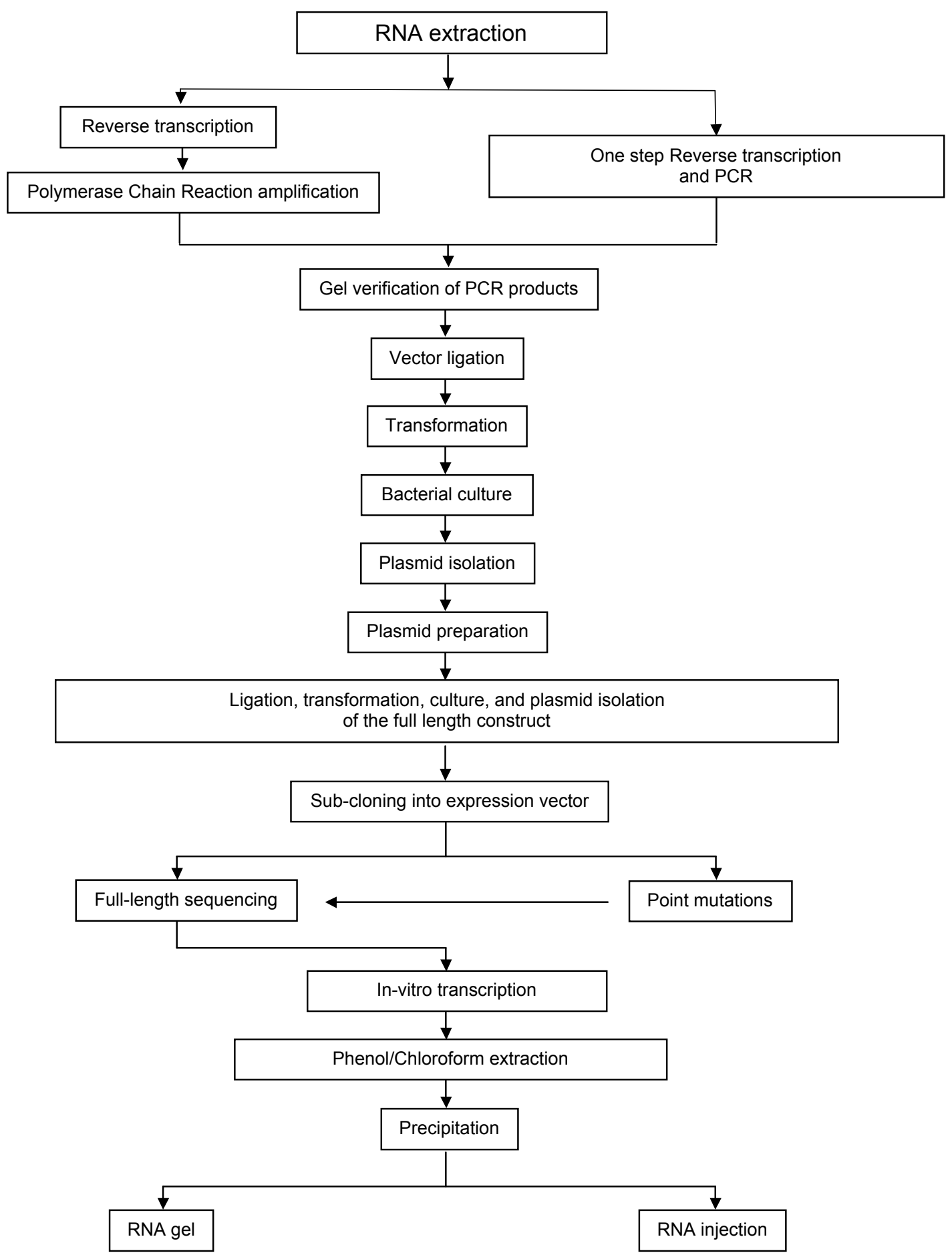

Figure 3. Standard cloning sequence scheme 
All cloning strategies including sequence analysis, primer design, restriction mapping, sequence alignments, and protein hydrophobicity plots were assisted by online analysis with the Laser gene software (DNAstar, Madison, USA).

\subsubsection{Tissue extraction}

Specimens of the species mus musculus were sacrificed in the Department of Molecular Biology of Neuronal Signals (Max-Planck-Institut für Experimentelle Medizin, Göttingen, Germany), by Mrs. Barbara Scheufler. Tissue samples from skeletal muscle, heart muscle and brain were surgically isolated followed by quick freezing in liquid nitrogen in order to avoid degradation. Samples were ground to a powder, adding more liquid nitrogen as necessary in order to prevent thawing and stored at $-80^{\circ} \mathrm{C}$ until further processing.

\subsubsection{RNA extraction}

The mouse tissues from which RNA was extracted were: skeletal muscle (rectus femoris muscle), heart muscle and total brain. Mice were healthy adults of NMRI strain that were born in captivity and kept under control conditions until sacrifice.

The extraction of total RNA was performed with the Qiagen RNAeasy Kit or with the Invitrogen Trizol reagent, both according to the instruction of the manufacturer.

PolyA ${ }^{+}-R N A$ was isolated using the FasTrack mRNA isolation kit from Invitrogen following the manufacturer instructions.

\subsubsection{Reverse transcription-Polymerase chain reaction, RT-PCR}

The technique consists of two parts: synthesis of cDNA from RNA by reverse transcription (RT) and amplification of a specific cDNA by Polymerase chain reaction 
(PCR). RT-PCR combines cDNA synthesis from RNA templates with PCR in order to provide a rapid, sensitive method for analyzing gene expression. The template for RT-PCR can be total RNA or poly $(A)+$ selected RNA. The RT reaction can be primed with random primers, oligo(dT), or a gene-specific primer (GSP) using a reverse transcriptase. RT-PCR can be carried out either in two-step or one-step formats. In two-step RT-PCR, each step should be performed under optimal conditions. The cDNA synthesis is performed first in RT buffer and one tenth of the reaction is removed and used for the PCR. High sensitivity (getting enough product from small samples) and high specificity (selective amplification of only the desired product) are the hallmarks of successful PCR.

\subsubsection{Reverse transcription "RT"}

RT was performed using Superscript II and III (Gibco BRL), using random

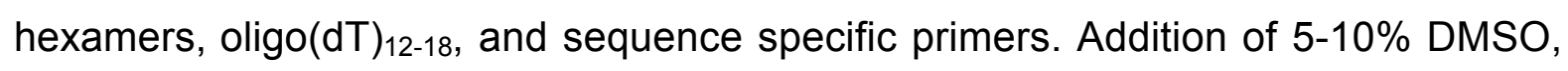
up to $10 \%$ glycerol, $1-2 \%$ formamide or combinations of these was assayed taking into account that the use of co-solvents lowers the optimal annealing temperatures of the primers (e.g. $5.5-6^{\circ} \mathrm{C}$ in $10 \%$ DMSO).

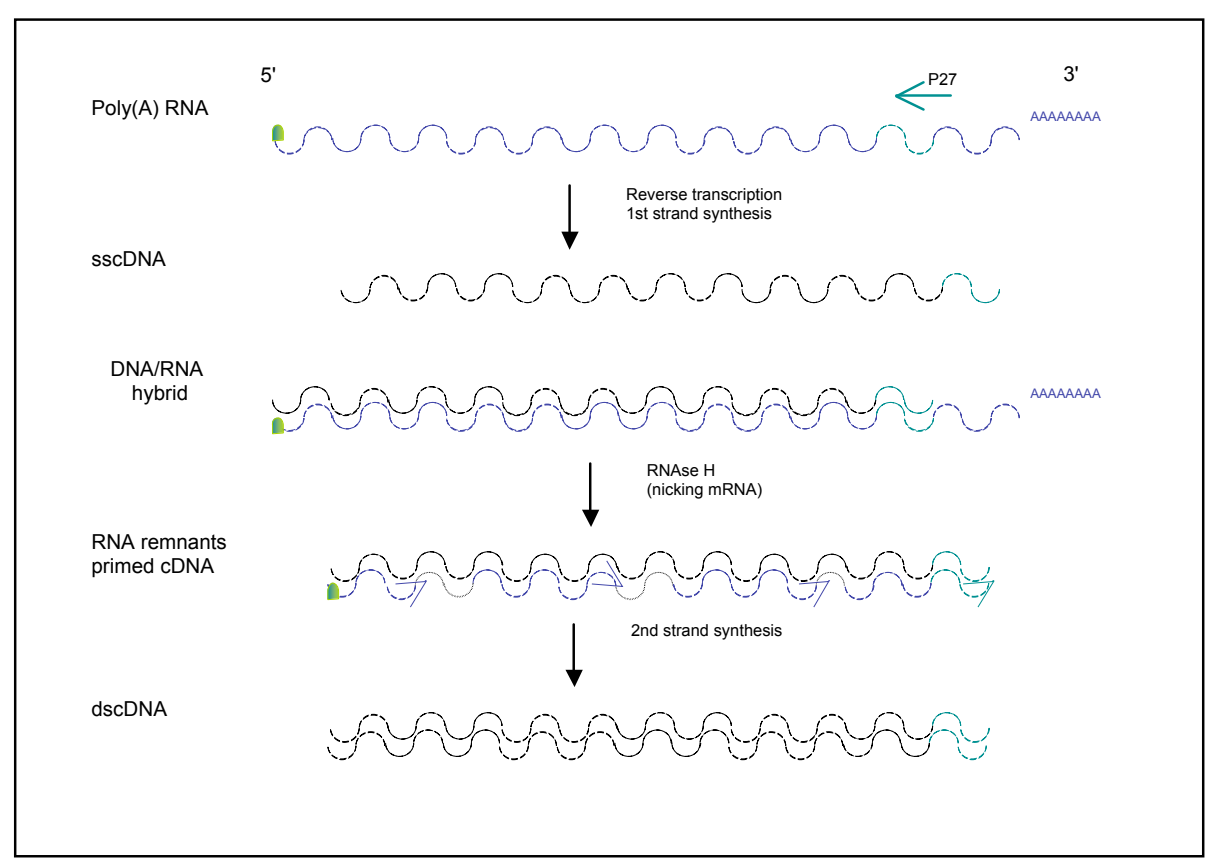

Figure 4. Reverse transcription scheme 


\subsection{Standard protocol for Oligo(dT) $)_{12-18}$ or GSP}

1-5 $\square \mathrm{g}$ total RNA

$1 \square \mathrm{l} \quad$ Oligo(dT) $12-18(0.5 \square \mathrm{g} / \mathrm{ml})$ or $2.0 \square \mathrm{M}$ GSP

1 I dNTPs (10 mM)

Sterile DEPC treated $\mathrm{H}_{2} \mathrm{O}$ to a final volume of $10 \mathrm{II}$

Incubate at $65^{\circ} \mathrm{C}$ for $5 \mathrm{~min}$

Transfer to ice for $1 \mathrm{~min}$

Add:

$2 \square \quad$ Reverse transcriptase buffer

4 ㄴ $25 \mathrm{mM} \mathrm{MgCl}_{2}$

$2 \mathrm{II} \quad 0.1 \mathrm{M} \mathrm{DTT}$

1 I I RNaseOUT recombinant RNase inhibitor

Mix carefully

Incubate at $42^{\circ} \mathrm{C}$ for $2 \mathrm{~min}$

Add 1 [I Reverse transcriptase (Superscript II, $50 \mathrm{U} / \square$ )

Mix carefully and incubate $50 \mathrm{~min}$ at $42^{\circ} \mathrm{C}$

Terminate the reaction by heating at $70^{\circ} \mathrm{C}$ for $15 \mathrm{~min}$.

Add $1 \mathrm{II}$ RNase $\mathrm{H}(2 \mathrm{U} / \square)$ and incubate at $37^{\circ} \mathrm{C}$ for $20 \mathrm{~min}$.

\subsection{Standard protocol for random hexamers}

$\begin{aligned} 1-5 \square \mathrm{g} & \text { total RNA } \\ 0.5 \square & \text { random hexamers }(50 \mathrm{ng}) \\ 1 \square & \text { dNTPs }(10 \mathrm{mM})\end{aligned}$

Sterile DEPC treated $\mathrm{H}_{2} \mathrm{O}$ to a final volume of $10 \square \mathrm{I}$

Incubate at $65^{\circ} \mathrm{C}$ for $5 \mathrm{~min}$

Transfer to ice for $1 \mathrm{~min}$ 
Add:

2 ㄴ Reverse transcriptase buffer

$4 \square \quad 25 \mathrm{mM} \mathrm{MgCl}_{2}$

2 ( $\quad 0.1 \mathrm{M} \mathrm{DTT}$

1 I RNaseOUT recombinant RNase inhibitor

Mix carefully

Incubate at $25^{\circ} \mathrm{C}$ for $2 \mathrm{~min}$

Add 1 II Reverse transcriptase (Superscript II, 50 U/ $\square$ )

Mix carefully and incubate at $25^{\circ} \mathrm{C}$ for $10 \mathrm{~min}$

Mix carefully and Incubate at $42^{\circ} \mathrm{C}$ for $50 \mathrm{~min}$

Terminate the reaction by heating at $70^{\circ} \mathrm{C}$ for $15 \mathrm{~min}$.

Add $1 \mathrm{I}$ RNase $\mathrm{H}(2 \mathrm{U} / \square)$ and incubate at $37^{\circ} \mathrm{C}$ for $20 \mathrm{~min}$

\subsection{RT protocol for GC rich targets}

1-5 $\square \mathrm{g}$ total RNA

$1 \square \mathrm{l} \quad$ Oligo(dT) $)_{12-18}(0.5 \square \mathrm{g} / \mathrm{ml})$ or $2.0 \square \mathrm{M}$ GSP

1 l $\quad$ dNTPs $(10 \mathrm{mM})$

Sterile DEPC treated $\mathrm{H}_{2} \mathrm{O}$ to a final volume of $25 \square \mathrm{I}$

Incubate at $65^{\circ} \mathrm{C}$ for $5 \mathrm{~min}$. Keep at $50^{\circ} \mathrm{C}$

Add:

4 Sterile DEPC treated $\mathrm{H}_{2} \mathrm{O}$

$5 \square \quad$ Reverse transcriptase buffer

10 ㄴ $25 \mathrm{mM} \mathrm{MgCl}_{2}$

$5 \square \quad 0.1 \mathrm{M} \mathrm{DTT}$

1 [ RNaseOUT recombinant RNase inhibitor

Warm to $50^{\circ} \mathrm{C}$.

Mix carefully both mixtures

Add $1 \square$ reverse transcriptase (Superscript II, $50 \mathrm{U} / \square$ ) 
Mix carefully and incubate at $50^{\circ} \mathrm{C}$ for $50 \mathrm{~min}$

Terminate the reaction by heating at $70^{\circ} \mathrm{C}$ for $15 \mathrm{~min}$.

Add $1 \mathrm{IINase} \mathrm{H}(2 \mathrm{U} / \square)$ and incubate at $37^{\circ} \mathrm{C}$ for $20 \mathrm{~min}$.

Alternatively, additives including glycerol and DMSO were added to first-strand synthesis reactions to help destabilize nucleic acid duplexes and melt RNA secondary structure. Up to $20 \%$ glycerol or up to $10 \%$ DMSO can be used without affecting SUPERSCRIPT II RT or M-MLV reverse transcriptase activity according to Gerard (Gerard, 1994). AMV RT will also tolerate up to $20 \%$ glycerol without loss of activity. For maximum RT-PCR sensitivity in SUPERSCRIPT II RT reactions, we added $10 \%$ glycerol and incubate at $45^{\circ} \mathrm{C}$. If one tenth of the RT reaction is added to the PCR, the final concentration of glycerol in the amplification reaction is $0.4 \%$, which will not inhibit PCR.

\subsubsection{Polymerase Chain Reaction}

PCR reactions were performed according to instructions for each Polymerase used.

\subsection{Taq Polymerase}

Reaction mixture:

$5 \mu \mathrm{l}$ 10x QIAGEN Taq PCR buffer*

$1 \mu \mathrm{l}$ dNTP mix (10 mM each)

$0.1-0.5 \mu \mathrm{M}$ sense primer

$0.1-0.5 \mu \mathrm{M}$ antisense primer

$0.5 \mu \mathrm{l}$ Taq DNA Polymerase (2.5 units/reaction)

Sterile DEPC distilled water

10 pg - $200 \mathrm{ng}$ Template DNA (aliquot from the reverse transcriptase reaction; $\leq 1 \mu \mathrm{g}$ cDNA/reaction)

Total volume $50 \mu \mathrm{l}$

* $\mathrm{Mg}^{2+}$ concentration was adjusted with $25 \mathrm{mM} \mathrm{MgCl}_{2}$ provided.

Additives: Q-solution 
Typical Qiagen Taq PCR cycling program was:

Initial denaturation: $3 \min 94^{\circ} \mathrm{C}$

3-step cycling

Denaturation: $0.5-1 \min 94^{\circ} \mathrm{C}$

Annealing: $0.5-1 \mathrm{~min} 50-68^{\circ} \mathrm{C}$; approximately $5^{\circ} \mathrm{C}$ below $\mathrm{Tm}$ of primers

Extension: $1 \mathrm{~min} 72^{\circ} \mathrm{C}$ For PCR products longer than $1 \mathrm{~Kb}$, increase the extension time by approximately 1 min per kb DNA.

Number of cycles: $25-35$.

Final extension: $10 \min 72^{\circ} \mathrm{C}$

\subsection{Pfx Polymerase}

Reaction mixture:

$5 \mu \mathrm{l} \quad$ 10X Pfx Amplification buffer*

$1 \mu \mathrm{l} \quad \mathrm{dNTP} \operatorname{mix}(10 \mathrm{mM}$ each)

$1 \mu \mathrm{l} \quad 50 \mathrm{mM} \mathrm{MgSO}_{4}$

$0.1-0.5 \mu \mathrm{M}$ sense primer

$0.1-0.5 \mu \mathrm{M}$ antisense primer

$1 \mu \mathrm{l} \quad$ Platinum ${ }^{\circledR}$ Pfx DNA Polymerase (2.5 units)

Sterile DEPC distilled water

10 pg - $200 \mathrm{ng}$ Template DNA (aliquot from the reverse transcriptase

reaction; $\leq 1 \mu \mathrm{g}$ cDNA/reaction)

Total volume $50 \mu \mathrm{l}$

* $\mathrm{Mg}^{2+}$ concentration was adjusted with $25 \mathrm{mM} \mathrm{MgSO}_{4}$ provided.

Typical Clontech Pfx Polymerase PCR cycling program was:

Initial denaturation: $2 \min 94^{\circ} \mathrm{C}$

3-step cycling

Denaturation: $15 \sec 94^{\circ} \mathrm{C}$

Annealing: $0.5-1 \mathrm{~min} 55^{\circ} \mathrm{C}$; approximately $5^{\circ} \mathrm{C}$ below $\mathrm{Tm}$ of primers

Extension: $1 \mathrm{~min} 68^{\circ} \mathrm{C}$. For PCR products longer than $1 \mathrm{~kb}$ increase the extension time by approximately 1 min per kb DNA Number of cycles: 25-35 
Final extension: $10 \min 68^{\circ} \mathrm{C}$

\subsection{BD Advantage 2 Polymerase}

Reaction mixture:

$5 \mu \mathrm{l}$ 10X BD Advantage 2 PCR buffer

$1 \mu \mathrm{l} \quad \mathrm{dNTP} \operatorname{mix}(10 \mathrm{mM}$ each)

$0.1-0.5 \mu \mathrm{M}$ sense primer

$0.1-0.5 \mu \mathrm{M}$ antisense primer

$1 \mu \mathrm{l}$ 50X BD Advantage 2 Polymerase Mix

Sterile DEPC distilled water

10 pg - $200 \mathrm{ng}$ Template DNA (aliquot from the reverse transcriptase reaction; $\leq 1 \mu \mathrm{g}$ cDNA/reaction).

Total volume $50 \mu \mathrm{l}$

Typical BD Advantage 2 Polymerase Mix PCR cycling program was:

Initial denaturation: $1 \mathrm{~min} 95^{\circ} \mathrm{C}$

3-step cycling

Denaturation: $15 \sec 95^{\circ} \mathrm{C}$

Annealing: $0.5-1 \min 55^{\circ} \mathrm{C}$; approximately $5^{\circ} \mathrm{C}$ below $\mathrm{Tm}$ of primers

Extension: $1 \min 68^{\circ} \mathrm{C}$. For PCR products longer than $1 \mathrm{~kb}$, increase the extension time by approximately 1 min per kb DNA.

Number of cycles: 25-35.

Final extension: $10 \min 68^{\circ} \mathrm{C}$

\subsubsection{One step Reverse transcription and PCR}

Superscript III OneStep RT-PCR (Invitrogen) system was used according to the directions of the manufacturer. 


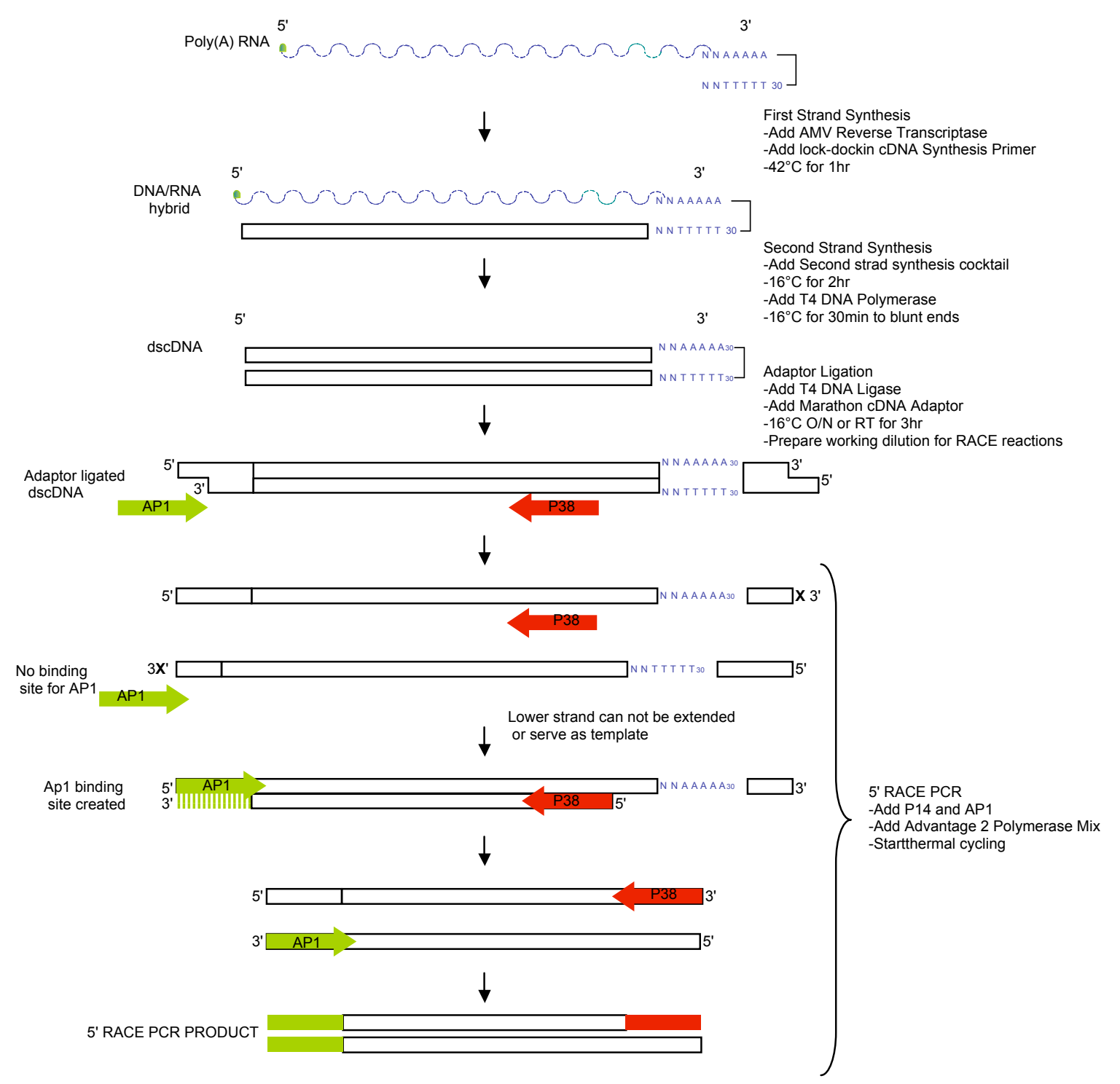

Figure 5. 5' RACE: First strand CDNA is synthesized using the gene-specific primer P38. Second-strand cDNA is synthesized according to Gubler and Hoffman (1983). cDNA is then amplified by PCR using primers P38 and AP1. For maximum sensitivity, the CDNA is further amplified by a second PCR using nested, specific primers P14 and AP1 (modified after Marathon cDNA Amplification kit User Manual, CLONTECH).

\subsubsection{Rapid amplification of cDNA ends "RACE"}

RACE PCR is used when information about DNA/protein sequence is limited. It enables the amplification of $3^{\prime}$ or $5^{\prime}$ ends of cDNAs generating fragments of cDNA with only one specific primer each and one adaptor primer. In order to obtain the Nterminus of the mouse kcna7 gene the 5' Marathon cDNA Amplification Kit (BD Clontech) was used according to the manufacturer. The specific strategy is summarized in Fig. 5. 


\subsubsection{Gel verification of the PCR products}

Nucleic acids were routinely separated by size on agarose slab gels via submarine gel electrophoresis. DNA has an inherent electrical charge that can be exploited to separate different size fragments on a horizontal gel within an electrical field. The mesh produced by the agarose gel provides the scaffold such that larger particles, though having a larger electrical charge, are impeded from progressing along the electrical field more than smaller particles that more freely pass through the meshwork. The resultant gel shows a ladder-like pattern in sample lanes when stained with ethidium bromide. Molecular size markers are run simultaneously in a marker lane so that estimated sizes can be calculated for each distinct rung on the sample ladder. An aliquot from all PCR reactions was run in an agarose gel $(0.7-1 \%$ depending on the product size) in order to verify the effectiveness of the amplification.

\subsubsection{Vector Ligation}

Once each fragment was amplified it was ligated into a cloning vector. For that purpose the vector had to be digested with the appropriate restriction enzyme(s) and cleaned with the QIAquick Nucleotide Removal Kit (Qiagen). The standard ligation enzyme used was T4 DNA Ligase (New England Biolabs). In a sterile PCR tube $1 \mu$ l $10 \times$ T4 DNA Ligase buffer, $1 \mu \mathrm{l}$ T4 DNA Ligase and the DNA solutions containing the fragments to be ligated were added to a final volume of $10 \mu \mathrm{l}$. The ligation reactions were incubated at $16^{\circ} \mathrm{C}$ overnight.

When pGem T-easy vector system was used, Taq Polymerase PCR products could be ligated directly with the ligase and specifications provided by the manufacturer.

\subsubsection{Transformation}

The bacterial strains used customarily were competent $\mathrm{DH} 5 \square$ and $\mathrm{XL} 1$-Blue depending on the vector used for each construct. The transformation protocol consisted in adding an aliquot of the DNA construct to a 200 l competent bacteria, 
incubate in ice for $30 \mathrm{~min}$, transfer to $42^{\circ} \mathrm{C}$ for 1 minute and incubated on ice for 3 more minutes.

\subsubsection{Bacterial culture}

The transformation mixture was plated into LB-agar plates containing the corresponding selection antibiotics and incubated at $37^{\circ} \mathrm{C}$ overnight.

When pBluescript or T-easy vector constructs were transformed onto XL1Blue bacteria the blue-white test was performed according to the standard procedure. Once grown in solid media, about 10 to 12 isolated colonies (when possible) were peaked and inoculated into $5 \mathrm{ml}$ LB medium supplemented with the selection antibiotic, and further incubated at $37^{\circ} \mathrm{C}$ overnight.

\subsubsection{Plasmid isolation}

The plasmid constructs were isolated from the fluid bacterial cultures using, either the Wizard Plus Minipreps DNA Purification System (Promega), or the Nucleospin plasmid isolation kit (Macherey-Nagel) according to the instructions. Identity of the fragments was tested through restriction digestion and visualized by agarose gel electrophoresis as described before.

\subsubsection{Plasmid preparation}

Positive constructs were further prepared for ligation into the full-length clone construct. Thus, positive constructs were restriction digested to generate the right ends, and cleaned.

\subsubsection{Restriction enzyme digestion}

In general, 0.25-2 \g DNA was digested in a total volume of $20 \square$, where $2 \square$ restriction enzyme buffer and 0.5-2 $\square$ I restriction enzyme were added. Some enzymes require supplementation with bovine serum albumin (BSA) to a final 
concentration of $100 \square \mathrm{g} / \mathrm{ml}$. Reactions were incubated at the optimal temperature for the corresponding enzyme for about 2-6 hours.

\subsubsection{Fill in}

In some cases a filling step using the DNA Polymerase I Large (Klenow) fragment from Promega was used to fill 5 ' protruding ends hence rendering blunt ends. The fill in protocol was performed according to the product instructions. In brief 1-4 $\mu \mathrm{g}$ of the digested DNA were mixed with $1 \mu \mathrm{l}$ Klenow $10 \mathrm{x}$ buffer, $40 \mu \mathrm{M}$ of each dNTP, $20 \mu \mathrm{g} / \mathrm{ml}$ acetylated BSA and 1 unit of Klenow Fragment per microgram of DNA, in a final volume of $20 \mu \mathrm{l}$. The reaction was incubated at room temperature for 10 minutes and stopped by heating the mixture at $75^{\circ} \mathrm{C}$ for 10 minutes.

When one enzyme ligation or blunt end ligation were performed an extra SAP dephosphorylation process was made.

\subsubsection{Shrimp Alkaline Phosphatase "SAP" dephosphorylation}

Dephosphorylation of DNA ends serves to prevent vector self re-ligation as well as avoiding self ligation of cohesive ends of long DNA fragments.

17 DNA solution

2 마 10x buffer

1 II SAP

Incubate at $37^{\circ} \mathrm{C}$ for 1 hour, Inactivate at $65^{\circ} \mathrm{C}$ for $15 \mathrm{~min}$

Stop reactions by heating at $65^{\circ} \mathrm{C}$ for $15 \mathrm{~min}$ or at $80^{\circ} \mathrm{C}$ for $20 \mathrm{~min}$ (if restriction endonuclease cannot be inactivated at $65^{\circ} \mathrm{C}$ ).

The corresponding ligation, transformation, culture, and plasmid isolation were performed as described for the partial fragments. 


\subsubsection{Sub-cloning into the oocyte expression vector pSGem}

The full-length clone construct was digested and ligated into the expression vector pSGem (generously provided by Dr. Michael Hollmann, Bochum, Germany). The poly-linker in this vector is flanked by the Xenopus globin 5' and 3' untranslated regions (UTRs), which has been reported to result in enhanced protein expression after injection of in vitro transcribed cRNA in the Xenopus laevis oocyte expression system (Liman et al., 1992).

\subsubsection{DNA Sequencing}

All constructs were sequenced using the dideoxy chain termination method with dye terminators on an Applied Biosystems 373 DNA sequencer (Applied Biosystems, Weiterstadt, Germany). Sequencing was performed by Mr. Fritz Benseler from the Department of Molecular Neurobiology, Max-Planck-Institut für Experimentelle Medizin, Göttingen, Germany.

\subsubsection{In-vitro transcription}

pSGem clones were linearized overnight with Nhe I. The linearization solution ran in agarose gels and the band corresponding to the linearized construct cut and extracted from the gel with the QIAEX II Gel Extraction Kit (Qiagen) according to the directions of the kit. The transcription was performed with the T7 Polymerase (Stratagene) according to:

$\begin{array}{ll}13 \text { linearized DNA } \\ 5 \square & \text { ATP (10 mM) Amersham } \\ 5 \square & \text { CTP (10 mM) Amersham } \\ 5 \square & \text { UTP (10 mM) Amersham } \\ 2.5 \square & \text { GTP (10 mM) Amersham } \\ 5 \square & \text { CAP (5 mM) Amersham }\end{array}$


10 미 $\quad 5$ buffer

$2.5 \square \quad$ RNasin (100 u) Promega

0.4 TI T7 RNA Polymerase

1.6 T7 RNA Polymerase dilution buffer

Final volume $50 \square$ l overall

Incubate at $37^{\circ} \mathrm{C}$ for 1 hour.

Add $5 \square$ I DNase in the tube.

Incubate at $37^{\circ} \mathrm{C}$ for $15 \mathrm{~min}$.

\subsubsection{Phenol/chloroform extraction}

Complete the volume of the transcription to 100 I with DEPC water

Add:

$100 \square$ phenol (1 volume)

100 chloroform (1 volume)

Vortex

Centrifuge at $13.200 \mathrm{rpm}$ for $3 \mathrm{~min}$

Separate the upper phase in a new tube (RNA tube)

Add $100 \square$ l of DEPC in the first tube (for 2nd extraction),

Vortex

Centrifuge at $13.200 \mathrm{rpm}$ for $3 \mathrm{~min}$

Separate the upper phase in the RNA tube

Add 200 l chloroform, vortex

Centrifuge at $13.200 \mathrm{rpm}$ for $3 \mathrm{~min}$

Separate the upper phase in a new RNA tube

\subsubsection{Precipitation}

Add:

20 I $\quad \operatorname{NaAC}(1 / 10$ volume $)$

600 EtOH absolute (3 volumes) 
Incubate at $-20^{\circ} \mathrm{C}$ for $12 \mathrm{~h}$

Centrifuge $30 \mathrm{~min}$ at $13.200 \mathrm{rpm}$

Wash with:

$500 \mathrm{ll} \quad 70 \% \mathrm{EtOH}$ (cold)

Centrifuge at $13.200 \mathrm{rpm}, 3 \mathrm{~min}$

Dry the pellet (SpeedVac, Eppendorf)

Re-suspend the pellet in $10 \square$ l sterile DEPC treated $\mathrm{H}_{2} \mathrm{O}$

\subsubsection{RNA gel electrophoresis}

In order to verify the size and quality of the cRNA an aliquot of the resuspended cRNA was ran in a RNA gel. Briefly, $0.45 \mathrm{~g}$ RNA agarose were dissolved in $34 \mathrm{ml}$ sterile DEPC treated $\mathrm{H}_{2} \mathrm{O}$ by heating the solution at $65^{\circ} \mathrm{C}$ (under the hood), then $4 \mathrm{ml} 10 \times$ MOPS and $7 \mathrm{ml}$ formaldehyde were added to the agarose solution and the gel was led for casting for at least 1 hour at room temperature.

\subsection{Cloning of kcna7 (mKv1.7)}

Three fragments were generated to clone mkcna7. Each fragment was processed independently and ligated together according to the scheme in figure 4 .

\subsubsection{Fragment F2-25}

Reverse transcription followed by Polymerase chain reaction: RT-PCR was used to amplify mouse kcna7 cDNA. RT from different mouse tissues was performed. Accordingly, skeletal muscle, heart muscle and total brain tissues were used for total

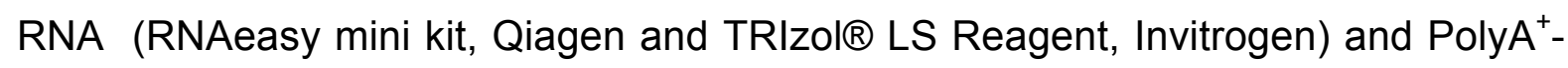
RNA extraction (FastTrack® 2.0 mRNA Isolation kit, Invitrogen). Self prepared RNAs as well as $\mathrm{BD}^{\mathrm{TM}}$ Premium Total mouse heart RNA (Clontech) were used as template for the first strand synthesis with Superscript II and III reverse transcriptases (Invitrogen). Synthesis was performed at standard conditions $\left(42^{\circ} \mathrm{C}\right)$ and at high 
temperature $\left(50^{\circ} \mathrm{C}\right)$. Gene-specific RT with primer P27 (P27-RT) was implemented after which a nested PCR with primers P23-P27 (for the first round of amplification), and P20-P21 (for the second amplification cycle) were performed. Such attempt offered good results in the amplification of a $\sim 1 \mathrm{Kbp}$ fragment from all three tissues assayed.

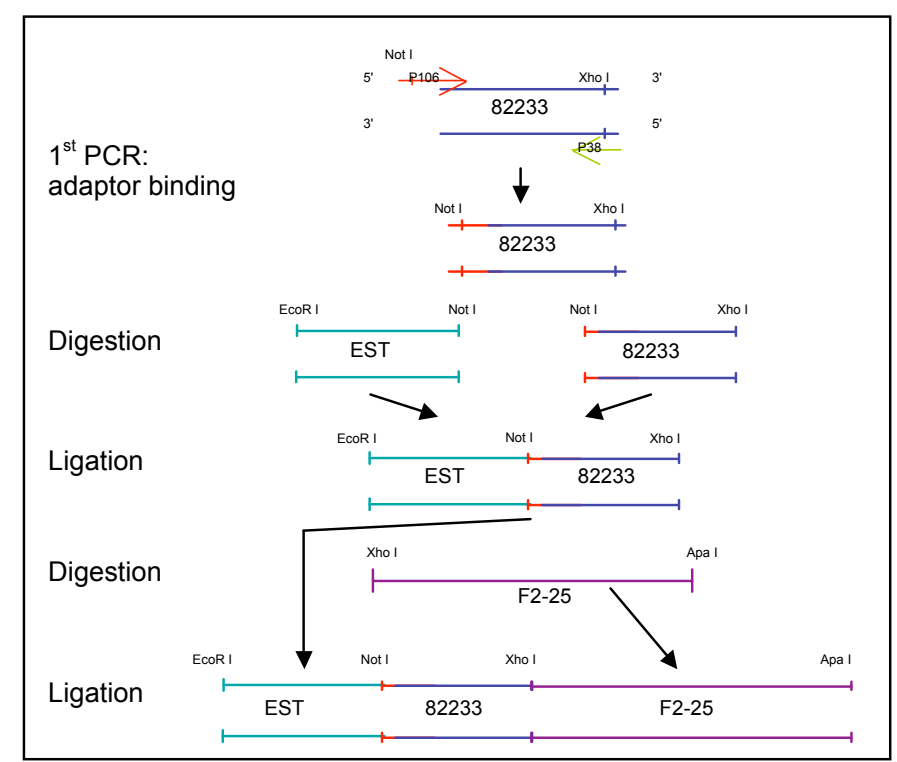

Figure 6. Adaptor ligation and full length cloning

\subsubsection{Fragment 82233}

5' RACE-PCR and high temperature/DMSO were designed and performed. The RACE principle and strategy followed with adaptor primer AP1 and P14 are described in the Fig. 5. From the RACE $\left(50^{\circ} \mathrm{C}, 10 \%\right.$ DMSO) products $164 \mathrm{bp}$ fragment named "82233" was isolated and further processed to incorporate in the full-length construct.

\subsubsection{Fragment EST}

EST corresponding to the $5^{\prime}$ part of the kcna7 gene (available in the public databases; RZPD IMAGp998P111081Q3) was bought. Even with the EST fragment a stretch of $18 \mathrm{bp}$ was still missing so the ligation scheme represented in Fig. 7 was performed. An adaptor primer P106 to the fragment 82233 was bound by PCR and 
therefore the missing piece including a restriction site for the ligation was introduced. The EST and the adaptor-82233 fragments were digested and ligated together.

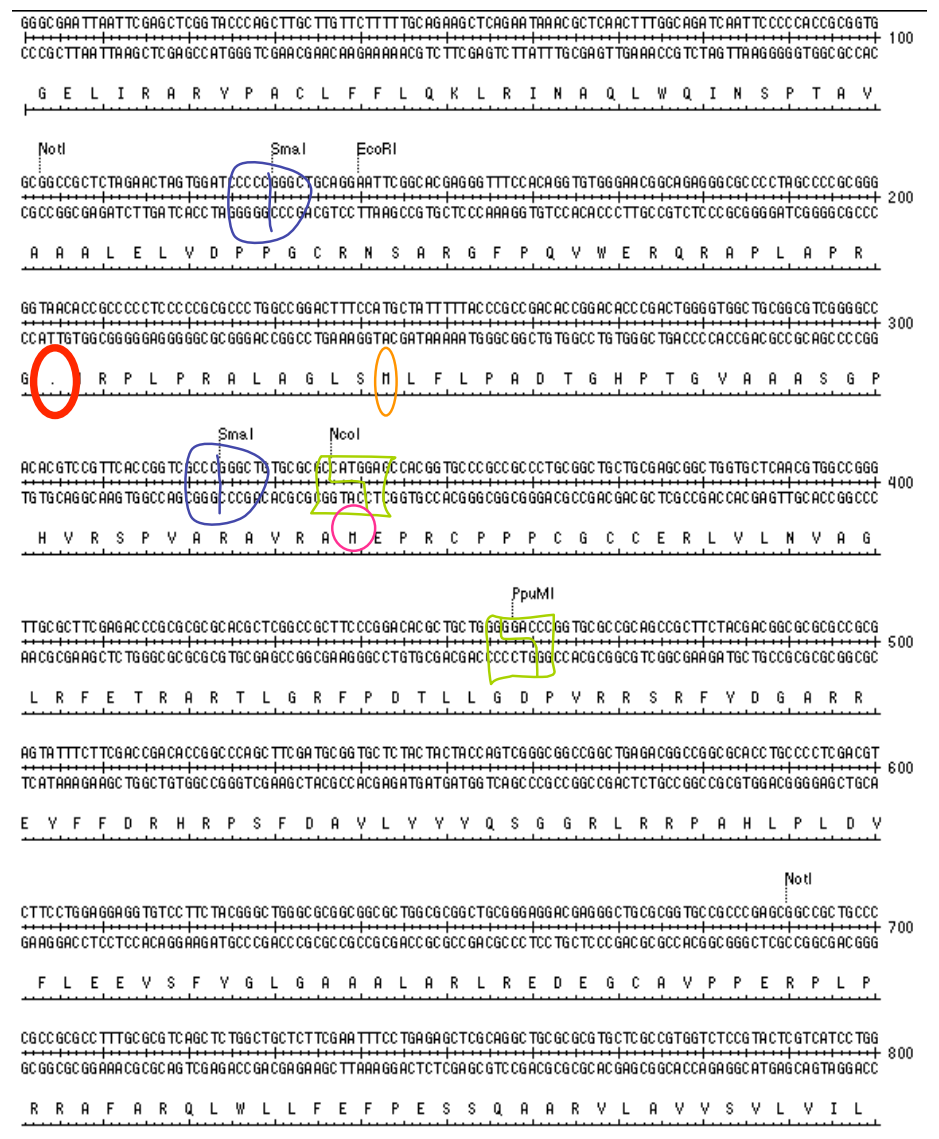

Figure 7. Restriction map of the first 800 nucleotides of mKv1.7. The restriction sites used are marked in blue for the mKv1.7-T0 construct and in green for the mKv1.7ロT1 clone. The red circle is around the stop signal in frame with the putative open reading frames. The ORF we propose is depicted within an orange ellipse. The ORF reported by Bardien et al., 2002 is inside the fuchsia circle.

\subsubsection{Amplification of kcna7 5' end}

One step RT-PCR (Advantage RT-PCR kit, Invitrogen) was used with primers P99-P38 to amplify a fragment of 670 bp. $2 \square \mathrm{M} \mathrm{MgSO}_{4}$ and 10\% DMSO were added to the reaction mixture. Accordingly, primers P186-P142 were used to amplify a fragment of $\sim 450 \mathrm{bp}$ after supplementation of the RT-PCR reaction mix with 2-2.4 $\square \mathrm{M}$ $\mathrm{MgSO}_{4}$ and $10 \%$ DMSO. Finally a 450bp fragment was amplified with primers P289-

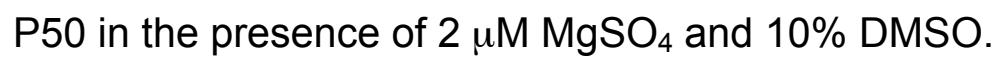




\subsection{5 mKv1.7-TO}

A N-terminal mutant lacking the first 32 amino acids of the mKv1.7 was generated by a restriction enzyme deletion (Sma I sites) over the full-length clone mKv1.7 (blue marks, Fig. 5). As a result a fragment of 194 (that contained the first start codon "ATG" in position 242-244) was deleted producing a protein with the ORF reported in the literature as the start of translation site. This mutant will be referred in this report as mKv1.7-T0.

\subsection{6 mKv1.7- $\Delta \mathrm{T} 1$}

In order to verify the importance of the tetramerization domain we generated the mKv1.7- $\mathrm{TT} 1$ construct consisting of the mKv1.7 with a fragment of 114 nucleotides, (38 amino acids, from aa 34 to 72 ) deleted from the predicted T1 domain. For that mutant the full-length construct was double digested with Nco I and Ppu M I (green marks, Fig. 5), the remaining ends were filled in with DNA Polymerase I (Klenow) large fragment (Promega) that rendered blunt ends. Finally, a blunt end ligation with ligase T4 was performed.

\subsection{Cloning of KCNA7 (hKv1.7)}

One step RT-PCR (Advantage RT-PCR kit, Invitrogen) was performed using human heart total RNA (Clontech) as template. The primers were designed according to the sequence published by Kashuba et al. (2001) under the accession number AJ310479. The cloning strategy consisted in the individual amplification of four fragments corresponding to hfrag1, hfrag-2, hfrag3, and hfrag4 (Fig. 6). hfrag 3 and 4 were successfully amplified with primers P222-P223 and P272-273 respectively using the standard conditions suggested by the manufacturer. hfrag 2 was amplified using primers P270-P271 only upon the supplementation of the reaction mix with $\mathrm{MgSO}_{4}$ to a final concentration of $2 \mathrm{mM}$ plus addition of pure DMSO to a final concentration of $10 \%(\mathrm{v} / \mathrm{v})$. Accordingly hfrag1 was amplified with primers 224-225, with 10\% DMSO: and $2.4 \mathrm{mM} \mathrm{MgCl}_{4}$. 
Once all the fragments were obtained the steps schematized in figure 6 were followed:

a. Frag1 was cloned directly in T-easy, then digested Sac I, and the Sac I digested frag2 was ligated in. This construct was called T-easy/frags $1+2$.

b. Frag2 was cloned in T-easy and digested Bstx I. Then Frag3 digested with Bstx I was ligated in. This construct was called T-easy/frags2+3.

c. Frag3 cloned into T-easy was digested Acc I, and the frag4 that was digested Acc I, introduced. This construct was called T-easy/frags3+4.

d. Construct T-easy/frags 2+3 was digested Nco I/Cla I and ligated into the T-easy/frag3+4 construct that was digested Nco I/Cla I.

e. Construct T-easy 1+2 was digested Nco I/Xho I was introduced into the 2+3+4 construct digested Nco I/Xho I.

f. pSGem vector was digested with Not I and then blunt ended with Polymerase I (Klenow) large fragment. pSGem 5'blunt end was digested with EcoR I.

g. Then the T-easy /hKv1.7 construct was digested Nco I and blunt ended with Polymerase I (Klenow) large fragment. KCNA7 5' blunt end was digested with EcoR I

h. Finally for joining hKv1.7 and the expression vector pSGem a ligation 5' blunt/3' EcoR I with ligase 4 was performed. 


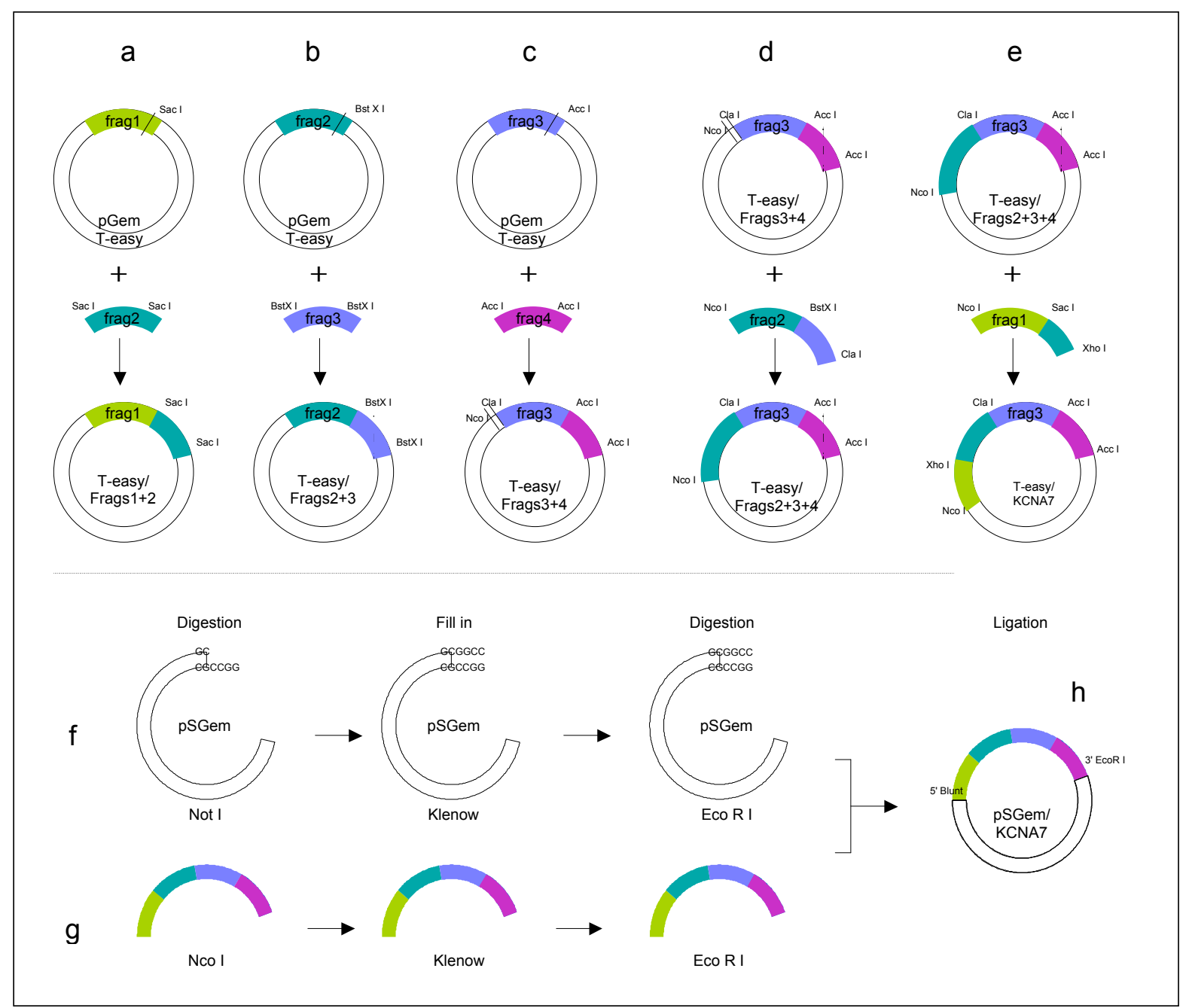

Figure 8. Cloning strategy for KCNA7

\subsection{Promoter Analysis}

The mouse kcna7 cDNA full-length sequence was analyzed with the Dragon Promoter Finder tool (Singapore, Bajic et al., 2003), and the GenomatixSuite promoter analysis software (Genomatix Software $\mathrm{GmbH}$, München, Germany).

\subsection{Protein Sequence Analysis}

The predicted amino acid sequences were analyzed with the help of the PSORT software (Nakai and Horton, 1999). This software employs the Klein et al.'s method to detect potential transmembrane segments (Klein et al., 1985; modified by 
Nakai and Kanehisa, 1992); as well the prediction of membrane topology method developed by Hartmann et al. (Hartmann et al., 1989).

\subsection{Electrophysiology methods}

\subsubsection{Oocyte extraction}

The Xenopus laevis oocyte heterologous expression system was used to study the electrophysiological characteristics of mouse and human Kv1.7 channels. The capacity to efficiently translate and express exogenous genetic material (Gurdon et al., 1971) coupled with the amenability of the giant oocyte cell for application of electrophysiology, provides compelling advantages for using Xenopus oocytes as an expression system to study protein function. The large size and small number of endogenous ion channels makes Xenopus oocytes an extremely useful model system for the study of ion channel activity using the two-electrode voltage clamp technique (TEVC). However, the size of the cell and the presence of invaginations in the membrane imply a big capacitance that makes the temporal resolution of TEVC limited in some kinetic studies. Accordingly, patch clamp experiments were performed.

Oocytes were surgically removed from anesthetized female Xenopus laevis specimens (20-30 min in $1.25 \mathrm{~g} / \mathrm{l}$ Tricaine solution, Fig. 7). Ovarian tissue contains immature and mature oocytes, as well as connective tissue from which the oocytes must be released. Defoliculation was performed by partial enzymatic digestion with Collagenase type $2(440 \mu / \mathrm{ml}$, Worthington Biochemical Corporation, Lakewood, USA), under shaking incubation at $17^{\circ} \mathrm{C}$ in a water bath (GFL 1083, Burgwedel, Germany) during $90 \mathrm{~min}$. Excessive collagenase exposure was avoided, therefore wash in Barth medium was preformed in order to stop the enzymatic reaction and oocytes from stages IV-VI were selected and allowed for restabilization for $24 \mathrm{~h}$ at $17^{\circ} \mathrm{C}$ in a standing incubator (Memmert ICP400, Schwabach, Germany). 

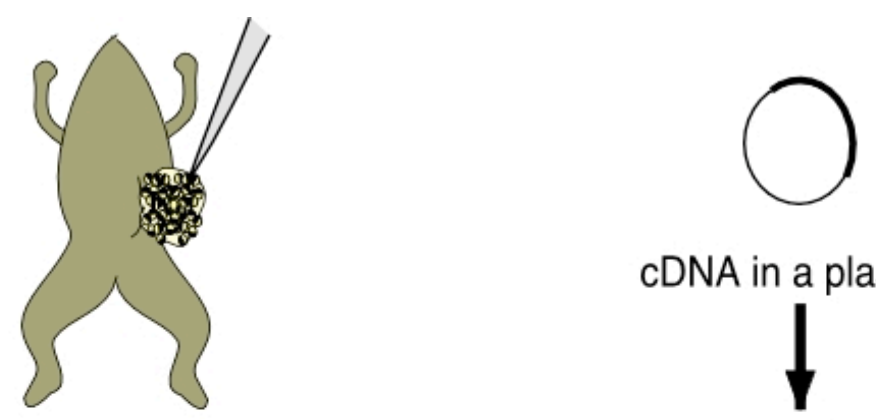

cDNA in a plasmid

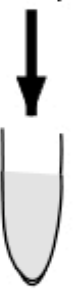

Remove ovaries from

Xenopus laevis

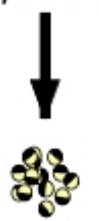

cRNA in vitro transcription

Separate oocytes and remove follicular layer with collagenase
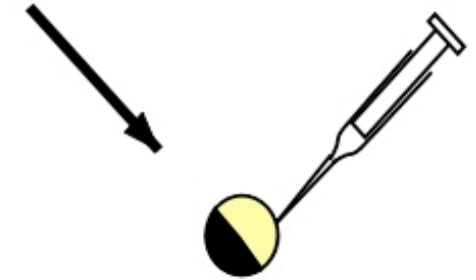

cRNA Microinjection
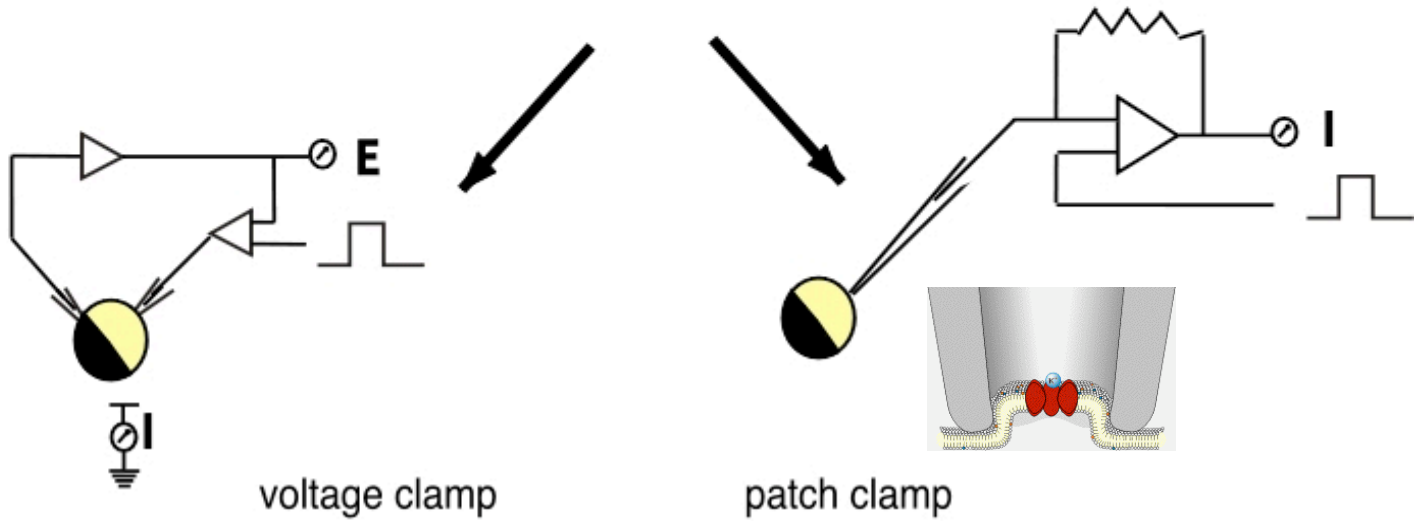

Figure 9. Xenopus laevis oocyte heterologous expression system. Ovarectomy, preparation of the oocytes and microinjection for electrophysiological recording.

\subsubsection{Microinjection}

cRNAs were generated by in vitro transcription as described in the molecular biology procedures section. Approximately, 750pgr cRNA (in 50nl) was injected using an automatic Drummond injector (Nanoject II, Broomal, USA) (Fig. 9). 
Microinjection needles were pulled from glass capillaries (Drummond 3-000203-G/X, Broomal, USA) using a standard vertical pipette puller PIP5 (HEKA, Lambrecht/Pfalz, Germany). Ideally the injection process should not distort the oocytes; needles tips were fired polished to avoid damage of the membrane of the oocytes and facilitate proper healing. To allow expression of the protein, injected oocytes were then incubated at $17.4^{\circ} \mathrm{C}$ in antibiotic supplemented Barth medium between 1 to 10 days prior to electrophysiological analysis. The antibiotics used were Cefuroxim/Zinacef750 (4mg/l, Aventis, Strasbourg, France) and Penicillin/Streptomycin (100 U/ml, Gibco, Carlsbad, USA). Attention was paid to alternate antibiotics in order to avoid the development of resistance.

\subsubsection{Two-electrode voltage clamp (TEVC)}

Whole cell currents were recorded with a two-electrode voltage clamp (TEVC) set up. During TEVC experiments one intracellular electrode measures the membrane potential (voltage electrode), and the second "current electrode" injects sufficient current to maintain the voltage clamped to the desired value (command potential) using a feedback circuit (Fig. 9). The amount of current that passes through the current electrode is the measured parameter and it is determined by the discrepancy between the membrane potential and the command potential.

The amplifier used for the voltage clamp recordings was a Turbo TEC-10 (Turbo Tec, npi electronics, Tamm, Germany) with electronic built-in series resistance compensation (Rs-comp). The electrical stimulation and registration of the current was performed through the EPC9 built-in ITC-16 AD/DA converter, controlled by a Macintosh G4 computer (Apple computer, Cupertino, $\mathrm{Ca}$, USA). The acquisition of data was made using Pulse software (HEKA, Lambrecht/Pfalz, Germany).

In TEVC experiments there is an extra resistance in "series" with that of the membrane recognized as series resistance (Rs). The Rs is due to the nature of all the elements that the injected current has to traverse within the oocyte: i.e. the bath, the cytoplasm, and the electrodes. When a current flows across the membrane, the 
Rs leads to a discrepancy between the measured membrane potential (controlled by the amplifier) and the "real" potential difference across the membrane. Artifacts caused by the series resistance deteriorate TEVC measurements considerably. For that reason, a proper Rs compensation is an important parameter in order to ensure good control over the potential. An electronic instrument used in conjunction with a standard TEVC amplifier was implemented to measure and display Rs automatically (for details see Polder et al., 2003).

\subsubsection{Patch clamp}

The patch clamp technique focuses in keeping the membrane potential across a small area of the membrane controlled. The technique performs this task by estimating the amount of current that is needed in order to maintain the membrane potential in "the patch" equal to that of the command circuit. The patch clamp amplifier is constituted mainly by a current-voltage converter (Fig. 9) that is capable of rapidly follow the variations of the command potential. By definition the injected current is proportional to the potential drop at the edges of the feedback resistance Rf.

The clean and smooth tip of a glass pipette is approached close to the surface of the cell membrane, usually applying negative pressure; this procedure frequently results in a very tight seal between the pipette and the membrane, isolating the patch of membrane confined to the inner diameter of the pipette tip. This first configuration is known as cell attached, and the currents flowing through the ion channels enclosed within the pipette tip are those measured by the patch-clamp amplifier. The "seal" obtained can reach resistances bigger than $10 \mathrm{G} \square$ where the tightness of the "gigaseal" prevents leak currents to flow between the pipette and the reference electrode.

After the cell attached configuration is obtained, the pipette can be pulledaway and the patch of membrane within the pipette tip gets excised from the entire cell, presenting the intracellular side of the membrane to the bath medium. This situation is recognized as the inside-out configuration. 
Once a cell attached patch is obtained, the patch of membrane within the tip of the pipette can be "broken" either by the application of negative pressure or by a brief voltage shock (zap), then the complete cell membrane is sealed to the edges of the pipette and the pipette solution is in direct contact with the cytosole generating the whole cell configuration. This configuration has the advantage that it gives control of the internal composition of the cell. Subsequently from "whole-cell" it is possible to achieve outside out that consists of a excised patch configuration where the extracellular side of the membrane is exposed to the bath solution. To achieve this the pipette should be withdrawn slowly from the cell allowing the patch of membrane at the tip to reseal with the internal surface facing the pipette solution. It is to mention that in the outside out configuration the pipette potential turns to be the same that the membrane potential therefore negative currents should be interpreted as cationic currents flowing into the cell, whereas positive currents result from outward cationic currents (Neher et al., 1978; Hamill et al., 1981; Moran, 1996).

In order to obtain high current density measurements we performed macro patches from large portions of oocytes membrane, using pipettes with a resistance between 0.7 and 1.5 $\mathrm{M} \square$, corresponding to a diameter tip of about 3-1.5 $\mu \mathrm{m}$.

\subsection{Instrumentation}

\subsubsection{Mechanical set-up and electrical recordings}

Mechanical stability and electrical shielding are crucial for a patch clamp setup. For that purpose the support system of the cell, the micromanipulators and the microscope (inverted microscope Zeiss Axiovert S100, magnification objectives 5X and 10X) were located on an antivibration table. This complete section of the set up is mechanically separated from the Faraday cage by a second table. Each individual component was grounded individually in order to obtain optimal shielding and at the same time avoiding current ("ground") loops between the instruments. 
For the control of the movements of the electrodes during TEVC experiments hydraulic micromanipulators MM33 (Märzhäuser-Wetzlar Gmbh, Germany) were used. The control of the patch clamp pipette was achieved with a stepper motor driven manipulator (Luigs and Neumann, Ratingen, Germany).

The amplifier used for the voltage clamp recordings was a Turbo TEC 10CD (Turbo Tec, npi electronics, Tamm, Germany) with an electronic built-in Rseriescompensation and for patch clamp experiments an EPC9 amplifier (HEKA Electronik, Lambrecht, Germany). In both techniques the electrical stimulation and registration of the current was performed through the EPC9 built-in ITC-16 AD/DA converter, controlled by a Macintosh, G3 microcomputer (Apple computer, Cupertino, Ca, USA).

For the exchange of bath solutions a gravity perfusion system with a mechanical valve (Hamilton Deutschland, Darmstadt, Germany) was used. This permitted the relative rapid exchange of up to 6 different solutions. The solution level of the recording chamber was maintained constant through a pressure driven level sensor system (Lorenz, Lindau, Germany).

\subsubsection{Micropipettes and electrodes}

TEVC microelectrodes were made from borosilicate filament glass capillaries (Hilgenberg, Malsfeld, Germany). After having been cut to the appropriate length, the edges of the glass capillary were fire rounded in order to improve the junction between the pipette and the pipette holder in the head-stage. This procedure also helps to protect the $\mathrm{Ag} / \mathrm{AgCl}$ coated electrode-wire from scratches produced by the rim of the glass. Capillaries for TEVC microelectrodes were pulled in two steps to the appropriate length and size in a vertical temperature controlled pipette puller PIP5 (HEKA, Lambrecht/Pfalz, Germany).

Patch clamp pipettes were made from borosilicate Kimax-51 glass capillaries (Kimble products, USA). The glass capillary pulled in two steps to the appropriate length and size in a vertical temperature controlled pipette puller LM3PA (List medicals, Germany). After pulling and right before use, pipettes were fire polished in 
order to smooth and clean the tips therefore improving the seal formation probability. Polishing was achieved by approaching the pipette tip close to a hot platinum wire installed in the microforge set/up.

To reduce noise characteristics and changes in the capacity of the pipette tips during solution exchanges, pipettes were coated with the polymer RTV (GE Bayer Silicones, Bergen, The Netherlands) that has low dielectric characteristics and it is highly hydrophobic. The fluid silicone layer was applied about $1 \mathrm{~mm}$ from the tip of the TEVC microelectrodes and $\sim 30 \mu \mathrm{m}$ away from the tip for patch pipettes. The polymer was hardened under a hot air stream avoiding any contact of the polymer with the cell membrane since it can leads to increases in the holding current during the experiments.

TEVC pipettes were filled with $2 \mathrm{M} \mathrm{KCl}$ and stored in a closed container under moist environment. Before use, the tip was broken to resistances between $500 \mathrm{KW}$ and $1 \mathrm{MW}$, corresponding to a tip diameter of 2-10 $\mu \mathrm{m}$. As long as the electrodes had low resistances they were reused for various experiments. The back end of each pipette was waxed to prevent creeping of the filling solution from the pipette to the electrode holder. Patch pipettes tip diameters ranged between 1 and 4MW. The patch pipette filling solution depended on the configuration that needed to be achieved therefore it changed according to the experiment.

\subsection{Stimulation Protocols and Data Analysis}

\subsubsection{IV-protocol}

Steady state activation curves and $\mathrm{RT}_{75}$, rise time to $75 \%$ of the peak current amplitude, were constructed with peak currents from IV protocols consisting of 500 ms depolarizing test potentials ranging from -60 to $+80 \mathrm{mV}$ in $10 \mathrm{mV}$ or $20 \mathrm{mV}$ increments (indicated in the corresponding experiments), holding potential $V_{h}=-100 m V$. The interpulse interval was15 sec. 
Inactivation kinetics for mKv1.7-T0 and hKv1.7-T0 was evaluated from IVs with $2.5 \mathrm{sec}$ pulse length.

Patch clamp IVs were as TEVC but of $100 \mathrm{~ms}$ and $150 \mathrm{~ms}$ duration pulses.

\subsubsection{Tail protocol}

The tail protocol consisted of 20 or $30 \mathrm{~ms}$ (mKv1.7 wt and mKv1.7-T0/hKv1.7, respectively) pulses to $+40 \mathrm{mV}$ and followed by $100 \mathrm{~ms}$ repolarizing pulses from -140 to $+40 \mathrm{mV}$ in $20 \mathrm{mV}, \mathrm{V}_{\mathrm{h}}=-100 \mathrm{mV}$. Interpulse interval of $15 \mathrm{sec}$.

\subsubsection{Repetitive stimulation protocols}

\subsubsection{Continuous pulse protocol}

Series of $12 \times 500 \mathrm{~ms}$ pulses to $+40 \mathrm{mV}$ from $\mathrm{V}_{\mathrm{h}}=-100 \mathrm{mV}$ were applied. The interpulse interval was $10 \mathrm{sec}$.

\subsubsection{Cumulative Inactivation protocol}

To evaluate cumulative inactivation trains of stimuli consisting of $8 \times 5 \mathrm{sec}$ depolarizations to $+40 \mathrm{mV}$ separated by $1 \mathrm{sec}$ periods at $V_{\mathrm{h}}=-100 \mathrm{mV}$, no leak subtraction $(p / n)$ protocol was implemented for this type of experiments.

\subsubsection{Double pulse protocols}

\subsubsection{Steady state inactivation}

Steady state inactivation curves were constructed from double pulse stimulation protocols consisting of a first $25 \mathrm{~ms}(\mathrm{mKv} 1.7 \mathrm{wt})$ or $40 \mathrm{~ms}$ (mKv1.7-T0 
and $h K v 1.7)$ depolarization to $+40\left(P_{1}\right)$ followed by hyperpolarizations from -120 to $+20 \mathrm{mV}$ in $10 \mathrm{mV}$ steps, and a second 25 or $40 \mathrm{~ms}$ pulse to $+40 \mathrm{mV}\left(\mathrm{P}_{2}\right)$. Intervals between $P_{1}$ and $P_{2}$ were 200, 250, 300, 500, 1000 and 1500 ms long.

The ratio between peak currents $P_{2} / P_{1}$ was plotted against the interpulse potential and resulting fitted to a Boltszmann function (see below).

\subsubsection{Recovery from inactivation}

Depolarizing pulses $P_{1}(4.5 \mathrm{sec})$ and $P_{2}(500)$ to $+40 \mathrm{mV}$ were applied, with repolarization to $-100 \mathrm{mV}$, the interpulse interval ranged from $2 \mathrm{~ms}$ to $8 \mathrm{sec}$. Each set of stimulation consisting of $P_{1}$, interpulse interval and $P_{2}$ was spaced from next one by $1 \mathrm{~min}$ at $-100 \mathrm{mV} . \mathrm{V}_{\mathrm{h}}=-100 \mathrm{mV}$.

\subsection{Leakage and capacitive current correction}

The currents measured during TEVC experiments were subtracted online with a standard P/n protocol (Heinemann et al., 1995). In a voltage range where voltage dependent channels are assumed not active, a scaled down version of the pulse protocol was applied $\mathrm{n}$ times and the resulting current was averaged, scaled and subtracted from that elicited by the main test pulse. A scaling factor of 0.25 was used and four leak responses were averaged to decrease the noise.

Current signals were sampled at 250-100 $\mu$ s (sampling rate: $4-10 \mathrm{kHz}$ ) for voltage clamp experiments and at $50 \mu \mathrm{s}$ (sampling rate: $20 \mathrm{kHz}$ ) for outside-out patches. The signals where low pass filtered with a Bessel filter at a frequency 4 times lower than the inverse of the sampling time, $1-2.5 \mathrm{kHz}$ for voltage clamp recordings and $5 \mathrm{kHz}$ for patch clamp recordings.

The cell membrane was kept at a holding potential $\left(\mathrm{V}_{\mathrm{h}}\right)$ of $-100 \mathrm{mV}$, where the probability for the channels under investigation to be in the closed state is assumed to be 1 . The pulse interval between two stimulations was always kept long enough to 
ensure re-equilibration of the channel states and recovery from any type of inactivation. In all TEVC experiments current signals were sampled at 250-100 $\mu$ s (sampling rate: 4-10 kHz), while patch clam experiments sampling was taken every 50 us (sampling rate: $20 \mathrm{kHz}$ ). Signals where low pass filtered with a Bessel filter at a frequency 4 times lower than the inverse of the sampling time, $1-2.5 \mathrm{KHz}$.

\subsection{Data analysis}

Off-line analysis was performed with a Macintosh G4 microcomputer (Apple, Cupertino, CA, U.S.A.) with Pulse Fit (HEKA, Germany) and Igor (Wavemetrics, Lake Oswego, USA) softwares.

\subsubsection{Macroscopic conductance}

The maximal current from test pulses from -60 to $+80 \mathrm{mV}$ were converted to conductances from:

$$
G_{K}=I_{K}^{*}\left(E_{m}-E_{K}\right)
$$

Where $G_{K}$ is the total potassium conductance; $I_{K}$ is the total current at the steady state; $E_{m}$ is the command potential and $E_{K}$ is the calculated reversal potential for each potassium concentration. $g$ values were normalized to maximal conductance and plotted against the potential, $g / V$ curves.

Normalized macroscopic conductance: $G_{K} / G_{\max }$

\subsubsection{Reverse potential}

$$
\mathrm{I}_{\mathrm{K}}=\mathrm{G}_{\mathrm{K}} /\left(\mathrm{E}_{\mathrm{m}}-\mathrm{E}_{\mathrm{K}}\right)
$$

Where $I_{K}$ was the peak current from the macroscopic tails. 


\subsubsection{Equilibrium potentials}

Equilibrium potential were calculated according to the Nernst equation

$$
E_{K}=R T / F * \operatorname{Ln}\left[K^{+}\right]_{0} /\left[K^{+}\right]_{i}
$$

With $R T / F=58.17 \mathrm{mV}\left(\right.$ at $20^{\circ} \mathrm{C}$ ). The same equation was used for the fit.

\subsubsection{Calculation of the $\mathrm{IC}_{50}$}

$\mathrm{IC}_{50}$ for each condition was calculated using peak current values at $+40 \mathrm{mV}$ according to the formula:

$$
I_{50}=\{\mathrm{fC} /(1-\mathrm{fc})\} *[\mathrm{Tx}] ; \quad \mathrm{fC}=\mathrm{I}_{\mathrm{Tx}} / \mathrm{I}_{\mathrm{Ctr}}
$$

Where: $f c$ is the fractional current; $[T x]$ the toxin concentration; $I_{T X}$, peak current under toxin; and $\mathrm{I}_{\mathrm{Ctr}}$, the peak current under control condition.

\subsubsection{Fits}

*Boltzmann fits were calculated according to the function:

$$
\mathrm{P}_{0} / \mathrm{P}_{\text {omax }} \text { or } \mathrm{G} / \mathrm{G}_{\max } \text { or } \mathrm{P}_{2} / \mathrm{P}_{1}=\text { Offset }+1 /\left(1+\exp ^{\left(\left(\mathrm{V}_{1 / 2}-\mathrm{V}_{\mathrm{m}}\right) / a\right)}\right)
$$

Where $V_{1 / 2}$ is the half activation or inactivation potential and $a$ the slope parameter that in the cases used in this work defines the voltage dependence.

*Mono exponential fits where calculated according to the function:

$$
y=y 0+A \exp \left(\operatorname{tau}^{-1 *} x\right)
$$

Where tau corresponds to $t a u_{\text {Inac }}$ or $\operatorname{tau}_{\mathrm{Cl}}$ 
${ }^{*}$ Double exponential fits were calculated according to the function:

$$
\mathrm{y}=\mathrm{A}_{0}+\mathrm{A}_{1}\left(\tan ^{-1}\right)+\mathrm{A}_{2}\left(\tan ^{-1}\right)
$$

Where $\operatorname{tau}_{1}$ and $\operatorname{tau}_{2}$ are the recovery from inactivation constants; and $A_{1}$ and $\mathrm{A}_{2}$ are the relative contribution parameters of each time constant.

The relative amplitudes were determined by:

$$
A_{1} /\left(A_{1}+A_{2}\right) ; A_{2} / A_{1}+A_{2}
$$

\subsection{Statistics}

Data is expressed as mean \pm standard error. Two tailed t-tests were used to evaluate the significance of the difference between means $(P<0.05)$ (Gossett, 1958). 


\section{RESULTS}

\subsection{Cloning of kena7}

Reverse transcription followed by polymerase chain reaction (RT-PCR) is a common strategy used to amplify specific cDNA products by PCR. RT from different mouse tissues was performed in order to clone the murine gene kcna7 corresponding to the mouse Kv1.7 voltage gated potassium channel protein. Primers were designed according to the sequence published in 1998 by Kalman et al. under the accession number AF032099 (Kalman et al., 1998). Total RNA is generally acceptable, though the use of polyA ${ }^{+}$-RNA enhances the ability to amplify rare messages. Accordingly, skeletal muscle, heart muscle and total brain tissues were used for total RNA and Poly ${ }^{+}-R_{N A}$ extraction. Self prepared RNAs as well as BD ${ }^{\mathrm{TM}}$ Premium Total mouse heart RNA (Clontech) were used as template for the first strand synthesis with Superscript II and III Reverse Transcriptases (Invitrogen). Synthesis was performed at standard conditions $\left(42^{\circ} \mathrm{C}\right)$ and at high temperature $\left(50^{\circ} \mathrm{C}\right)$. Either oligo $(\mathrm{dT})_{12-18}$, random hexamers and gene specific primers are customarily used to prime the reverse transcription reaction. We started using random hexamers and oligo(dT)12-18 however the PCRs performed with the generated cDNAs were not successful. The use of gene-specific primers is known to eliminate unwanted endogenous random priming that occurs during standard cDNA synthesis reactions; thus gene-specific priming typically maximizes the yield of target cDNA while limiting the complexity of the cDNA "library" synthesized. RT with primer P27 (P27-RT) was implemented after which a nested PCR (strategy shown in the inset of Fig.10) with primers P23-P27 (for the first round of amplification), and P20P21 (for the second amplification cycle) were performed. Such attempt offered good results in the amplification of a $\sim 1 \mathrm{Kbp}$ fragment from all three tissues assayed. The fragment of 1188bp was obtained from the first PCR and a further fragment of 999bp from the second (Fig.10). Isolation and cloning of the F2-25 fragment allowed sequencing of the cDNA. F2-25 proved to be $99 \%$ homologous to the $3^{\prime}$ end of the kcna7 cDNA (AccNum AF032099) corresponding to a protein fragment that spans from the transmembrane segment $S 2$ to the end of the C-terminus (Fig. 11). 


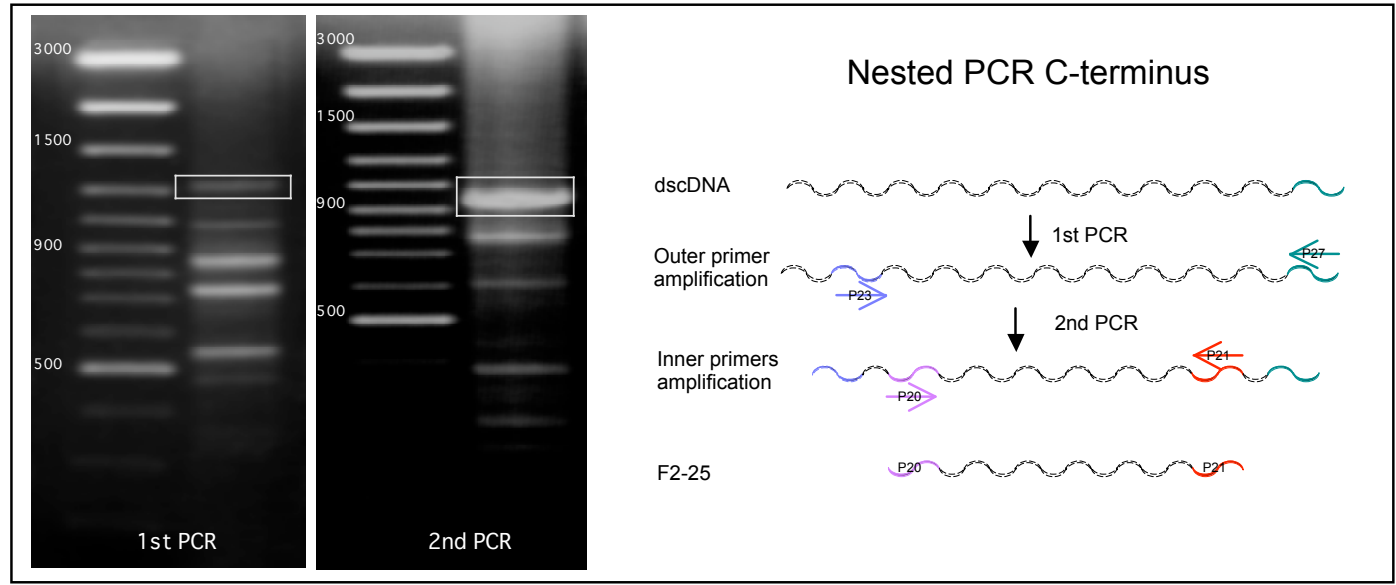

Figure 10. Nested PCR for C-terminus of mouse kcna7. Agarose gel electrophoresis of the nested PCR products. 1st PCR: gel containing the PCR product generated with primers P23-P27. 2nd PCR displays PCR products obtained with primers P20-P21. The positive fragment is within the box. The inset shows strategy used for the nested PCR.

Disappointingly the amplification of the $\sim 700 \mathrm{bp}$ corresponding to the $5^{\prime}$ end of the kcna7 gene turned out to be elusive to the RT-PCR strategies undertaken for the 3' end. Neither primer specific RT (with primers P14, P27, P38, P50) nor high temperature-RT offered good results. Addition of secondary structure releasing agents (co-solvents) like glycerol (up to $10 \%$ ), DMSO (2.5-10\%), as well as $1-2 \%$ formamide, or mixtures of these, was not helpful in the reverse transcription of the $5^{\prime}$ end of kcna7. A systematic combination of each condition was tried with no positive result. We performed analysis of the three-dimensional configuration of the whole mRNA molecule and specifically of the region of RNA that we could not reverse transcribe using GeneBee-Net v.2.0 software (1994-2003). Figure 12 shows the predicted secondary structure of the $488 \mathrm{bp}$ of mRNA corresponding to the 5 ' end of the kcna gene (from +1 to 488). As it can be observed the calculated free energy for the loop formed by the stretches between nucleotides 394-403 and 471-462 (Stem1) reaches $-26.4 \mathrm{Kcal} / \mathrm{mol}$ (area inside the circle in Fig.12A). This is the highest value observed over the fragment, moreover the highest $\square G^{\circ}$ calculated on the whole mRNA molecule (Fig.12B). The plot of the calculated $\square G^{\circ}$ at $37^{\circ} \mathrm{C}$ includes the free energy of the pairing between different stretches of the mRNA (Fig.12B). Actually it is evident that the regions that pair most stably lay in the $5^{\prime}$ end of the molecule (trapezoid) therefore making it very difficult for the polymerases to pass through. 
GATGACTACAAGGGAAAGTCAAGAGATCCACGGAAAAGCGCCGGGTGGCAGTGTTTCCACAGGTGTGGGAACGGCAGAGGGCGCCCCTAGCCCCGCGGG -42 P186

GGTAACACCGCCCCCTCCCCCGCGCCCTGGCCGGACTTTCCATGCTATTTTTACCCGCCGACACCGGACACCCGACTGGGTGGCTGCGGCGTCGGGGCC 059

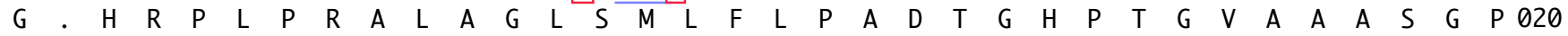
P99

ACACGTCCGTTCACCGGTCGCCCGGGCTGTGCGCGCCATGGAGCCACGGTGCCCGCCGCCCTGCGGCTGCTGCGAGCGGCTGGTGCTCAACGTGGCCGGG 159

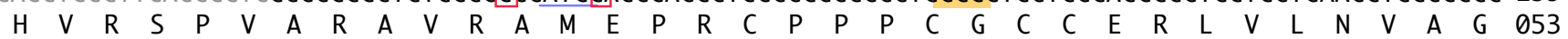
TTGCGCTTCGAGACCCGCGCGCGCACGCTCGGCCGCTTCCCGGACACGCTGCTGGGGGACCCGGTGCGCCGCAGCCGCTTCTACGACGGCGCGCGCCGCG 259

$\begin{array}{lllllllllllllllllllllllllllllllllllll}L & R & F & E & T & R & A & R & T & L & G & R & F & P & D & T & L & L & G & D & P & V & R & R & S & R & F & Y & D & G & A & R & R & 086\end{array}$ AGTATTTCTTCGACCGACACCGGCCCAGCTTCGATGCGGTGCTCTACTACTACCAGTCGGGCGGCCGGCTGAGACGGCCGGCGCACCTGCCCCTCGACGT 359 $\begin{array}{llllllllllllllllllllllllllllllllllllllllll}E & Y & F & F & D & R & H & R & P & S & F & D & A & V & L & Y & Y & Y & Q & S & G & G & R & L & R & R & P & A & H & L & P & L & D & V & 120\end{array}$

$$
+\quad P 142
$$

CTTCCTGGAGGAGGTGTCCTTCTACGGGCTGGGCGCGGCGGCGCTGGCGCGGCTGCGGGAGGACGAGGGCTGCGCGGTGCCGCCCGAGCGGCCGCTGCCC 459

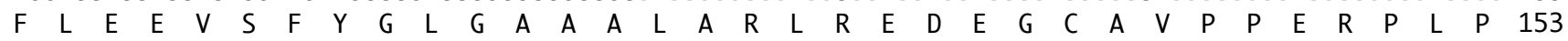
P106 >

$$
<\stackrel{\mathrm{P} 50 \mathrm{P} 23}{\longrightarrow}
$$

CGCCGCGCCTTTGCGCGTCAGCTCTGGCTGCTCTTCGAATTTCCTGAGAGCTCGCAGGCTGCGCGCGTGCTCGCCGTGGTCTCCGTACTCGTCATCCTGG 559

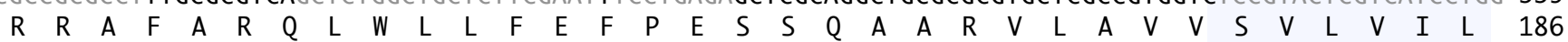

$\mathrm{P} 20>$ P14

TCTCCATCGTGGTCTTTTGCCTCGAGACACTGCCAGACTTCCGCGACGACCGCGATGACCCGGGGCTCGCGCCCGTAGCCGCTGCTACTGGCCCGTTCCT 659 $\begin{array}{lllllllllllllllllllllllllllllllllll}V & S & I & V & V & F & C & L & E & T & L & P & D & F & R & D & D & R & D & D & P & G & L & A & P & V & A & A & A & T & G & P & F & L & 220\end{array}$ P38

CGCCCGACTGAATGGCTCCAGTCCCATGCCAGGAGCCCCTCCCCGACAGCCCTTCAACGATCCATTCTTTGTGGTGGAGACCCTGTGTATCTGCTGGTTC 759 $\begin{array}{llllllllllllllllllllllllllllllllllllllllll}A & R & L & N & G & S & S & P & M & P & G & A & P & P & R & Q & P & F & N & D & P & F & F & V & V & E & T & L & C & I & C & W & F & 253\end{array}$ TCCTTTGAGCTGCTGGTGCGTCTGGTGGCCTGCCCTAGCAAAGCTGTGTTCTTCAAGAATGTGATGAACCTAATTGACTTCGTGGCCATCCTGCCTTACT0859

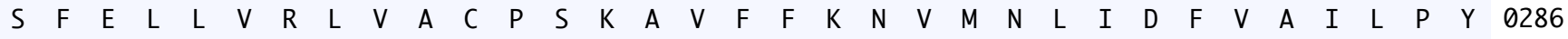
TCGTGGCCCTGGGCACGGAGTTAGCCCGGCAGCGGGGTGTGGGCCAGCCGGCTATGTCCCTGGCCATCCTAAGGGTCATCCGATTGGTGCGTGTCTTCCG0959 $\begin{array}{llllllllllllllllllllllllllllllllllll}F & V & A & L & G & T & E & L & A & R & Q & R & G & V & G & Q & P & A & M & S & L & A & I & L & R & V & I & R & L & V & R & V & F & R 0320\end{array}$ CATCTTCAAGCTCTCCAGGCATTCGAAGGGTCTACAGATCTTGGGTCAGACACTGCGGGCTTCCATGCGTGAGCTAGGTCTCCTCATCTTCTTCCTCTTC1159

$\begin{array}{lllllllllllllllllllllllllllllllllll} & \text { I } & F & K & L & S & R & H & S & K & G & L & Q & I & L & G & Q & T & L & R & A & S & M & R & E & L & G & L & L & I & F & F & L & F & 0353\end{array}$ ATTGGCGTGGTCCTCTTTTCCAGCGCAGTCTACTTTGCTGAAGTGGACCGGGTGGACACCCATTTCACCAGCATCCCGGAGTCCTTTTGGTGGGCAGTGG1259 $\begin{array}{lllllllllllllllllllllllllllllllllllllllll}I & G & V & V & L & F & S & S & A & V & Y & F & A & E & V & D & R & V & D & T & H & F & T & S & I & P & E & S & F & W & W & A & V & 0386\end{array}$ TCACCATGACCACGGTTGGCTATGGGGACATGGCACCCGTCACCGTGGGTGGCAAGATCGTGGGCTCTCTGTGTGCCATTGCAGGTGTGCTCACCATCTC1359

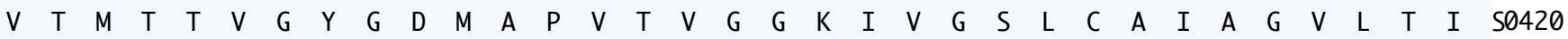
TCTGCCTGTGCCTGTCATTGTCTCTAACTTTAGCTACTTTTACCACCGGGAGACAGAGGGCGAAGAGGCAGGGATGTACAGCCATGTGGACACACAGCCC1459

$\begin{array}{llllllllllllllllllllllllllllllllll}L & P & V & P & V & I & V & S & N & F & S & Y & F & Y & H & R & E & T & E & G & E & E & A & G & M & Y & S & H & V & D & T & Q & P & 0453\end{array}$ $<P 21$

TGCGGTACCCTGGAGGGCAAGGCTAATGGGGGGCTGGTGGACCCTGAGGTGCCTGAACTCCTCCCACCACTCTGGCCCCCTGCAGGGAAACACATGGTGA1559

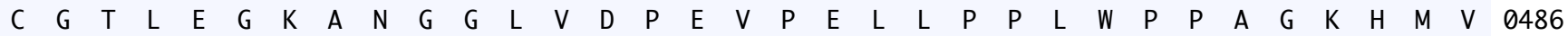
$<\quad$ P21

CTGAGGTGTGAGGGTCAACTGGGGTCTCCAGGGAGCAGTGGGGTGGGAGGGAGGAGGGAAGGCAGCTTATGTGACAGGAATTGCCAGGATTTGGGTTGTG1659 $\mathrm{T} \mathrm{E} V \bullet \quad 0489$ TCCAGGGCTCCCCCCATTGATTGCGCAATGTGGTAGAGCTGTGCAAATGTCCAGGGGTCAGGTGCTGGGTTAAGGACTAAGATGGTAACAAAAGGGTACA1759 P27

GATTCTTAATCTGAAGGCATGTCACATGGTGGCTCCTAGGGGGACGGCTCTATGGGTAGCACTGTAAGAGACTTGGCCATAGATTGTGAGTTTCCTAGGT1859 GTTCGTGGGGTCCCACTGGGGGCAAGTGTGGTCCCGTTAACACTTGATAGGTACTAATAGAACTTTGGGTCCTCTAGGGCCAAGTCGGCTCCTGTAGGTC1959 TCTGGAGCACTGTTAAAGAGTGTGAGCTTGTGGCTACATGGTCACACACAGAGGTTATGTGGTGATACATAGCAGATTGTGTGGATTCATGTTTCTTCTA2159

Figure 11. cDNA and amino acid sequences of kcna7. The primers used are indicated with the arrows and corresponding light gray font. Start codons are underlined in blue. Positions -3 and +4 are within red boxes. The orange solid box marks the area of conflict with the sequence of Kalman et al. 1998. In the gray box is shown the fragment corresponding to F2-25. 
Free Energy of Structure $=-180.7 \mathrm{kkal} / \mathrm{mol}$

A.

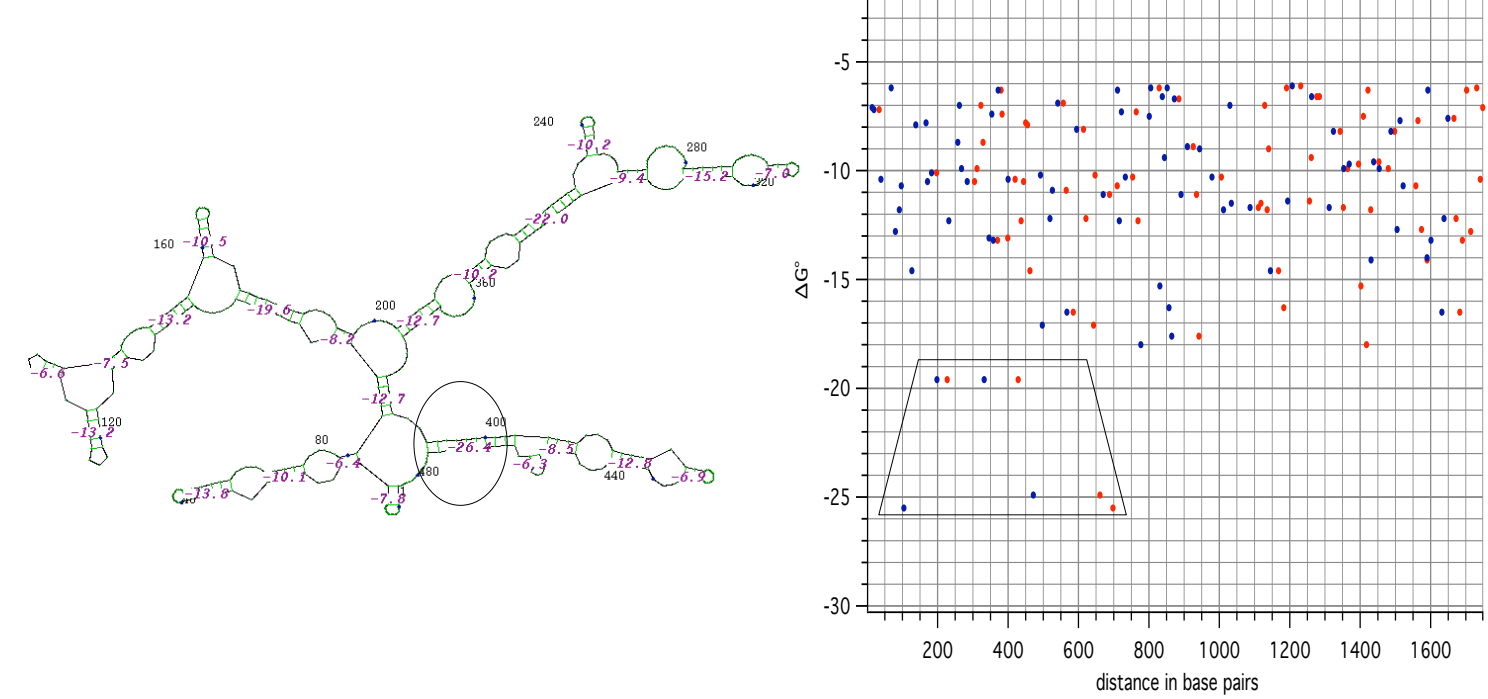

Figure 12. Secondary structure of the kcna7 RNA. A. Predicted secondary structure from calculations of free energy at $37^{\circ} \mathrm{C}$. The circle remarks the area of higher stability (lower $\square \mathrm{G}$ ). $\mathrm{B}$. Plot of the free energy values for the complete RNA molecule, predicted hybridizing pairs are shown as red and blue dot pairs located at the same $\square G^{\circ}$ level ( $Y$ axis).

In situations in which the ends of the genes are unknown or hard to obtain it is customary to attempt rapid amplification of CDNA ends (RACE). Indeed, standard 5' RACE-PCR and high temperature/DMSO RACE-PCR were designed and performed. The RACE principle and strategy followed is given in the methods section. Figure 13 shows the picture of agarose gel electrophoresis from the products obtained when the adaptor primers AP1 and P14 were used (Fig.13). No cDNA fragment of the expected length $-745 \mathrm{bp}$ - could be visualized in any of the RACEs performed. Nevertheless after several attempts were made each fragment obtained was sequenced in order to try to identify the source of the problem. Most of the RT-PCR products aligned within a stretch of 164bp that had in the 3 ' end the sequence of primer P14 corresponding to kcna7. This fragment was termed 82233 but upstream of this region the sequences of the amplified products were not consistent with the target gene. This suggested that the reverse transcription and/or the PCR polymerases were failing to proceed upstream of the 82233 region that corresponds to nucleotide 460 in Fig. 10. 


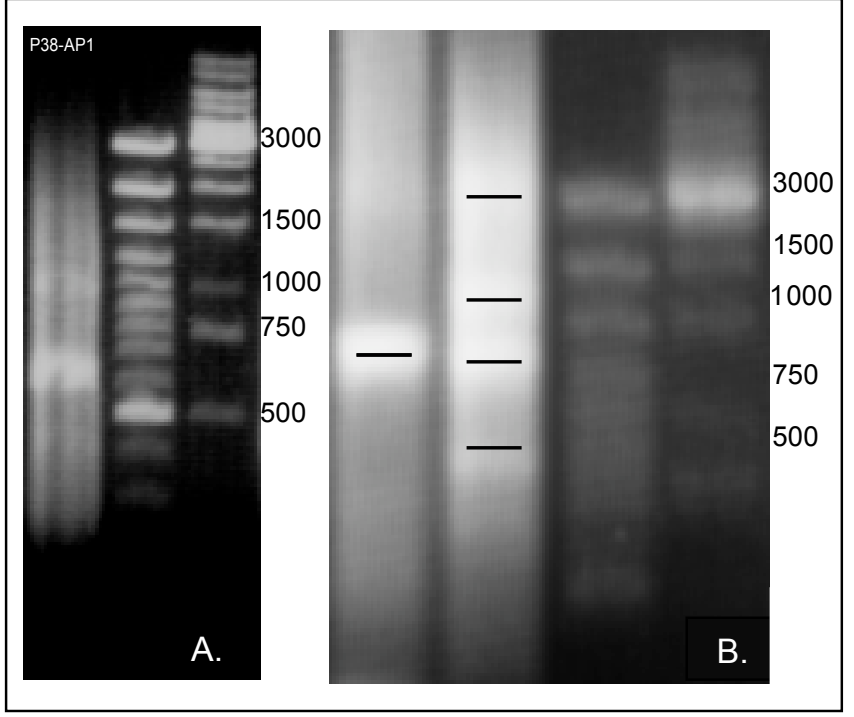

Figure 13. Agarose gel electrophoresis of the RACE products. A. Product from the first PCR. B. Product from the second PCR.

Since the PCR amplified cDNA did not contain the full open reading frame (ORF) of the mKv1.7 protein and in order to complete our clone we acquired the mouse EST corresponding to the 5' part of the kcna7 gene (available in the public databases; RZPD IMAGp998P111081Q3). Even with the EST fragment a stretch of 18bp was still missing so the ligation scheme represented in Fig.6 (in the methods section) was performed; thus the first full-length cDNA construct of kcna7 was completed.

Once obtained, the full-length cDNA construct was fully sequenced. The nucleotide alignments of the AF032099 cDNA clone (Kalman et al., 1998); AJ409348 mouse cDNA fragment and human cDNA clone AJ310479 (Kashuba et al., 2001); EST genomic fragment IMAGp998P111081Q3; the AC073711 (a working draft sequence of the mouse chromosome 7); and our sequence revealed puzzling difference in the putative ORFs of the mouse kcna7 gene. According to our prediction ORF prediction software there is a premature AUG codon in frame with the rest of the sequence. This premature AUG is present in sequences AJ409348 mouse cDNA fragment, EST genomic fragment IMAGp998P111081Q3, genomic draft of chromosome 7 AC073711, and our clone.

In order to clarify the location of the ORF of the mouse kcna7 gene we performed one step RT-PCR (Superscript III OneStep RT-PCR, Invitrogen) in the presence of different $\left[\mathrm{Mg}^{2+}\right]$ and maximal concentrations of DMSO from mouse 


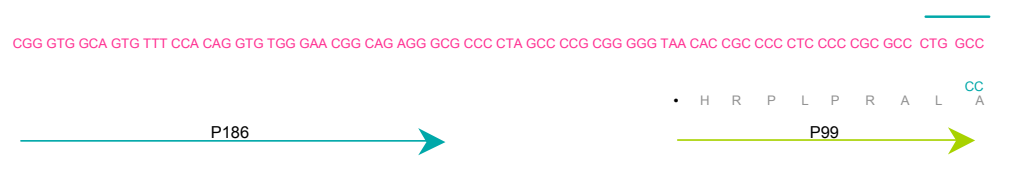

GGA CTT TCC ATG CTA TTT TTA CCC GCC GAC ACC GGA CAC CCG ACT GGG GTG GCT GCG GCG TOG GGG CCA CAC GTC CGT TCA CCG GTC GCC GGA CTT TCC ATG CTA TTT TTA CCC GCC GACACC GGA CAC CCG ACT GGG GTG GCT GCG GCG TCG GGG CCA CAC GTC CGT TCA CCG GTC GCC

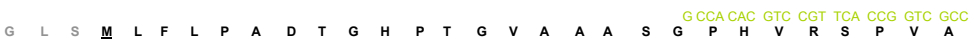

CGG GCT GTG CGC GCC ATG GAG CCA CGG TGC CCG CCG CCC TGC GGC TOC TGC GAG CGG CTG GTG CTC AAC GTG GCC GGG TTG CGC TTC GAC CGG GCT GTG CGC GC

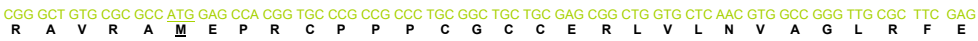

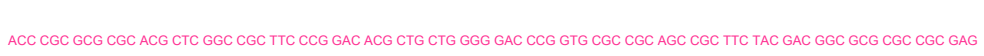
ACC CGC GCG CGC ACG OTC GGC CGC TTC CCG GAC ACG CTO CTG GGG GAC CCG GTG CGC CGC AGC CGC TTC TAC GAC GGC GCG CGC COC GAC

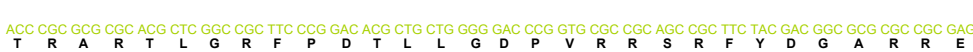

TAT TTC TTC GAC CGA CAC $\triangle G G$ CCC AGC TTC GAT GCG GTG CTC TAC TAC TAC CAG TCG GGC GGC CGG CTtG AGA CGG CCG GCG CAC GTG CCC TAT TTC TTC GAC CGA CAC CGG CCC AGC TTC GAT GCG GTG CTC TAC TAC TAC CAG TCG GGC GGC CGG CTG AGA CGG CCG GCG CAC GTG CCC

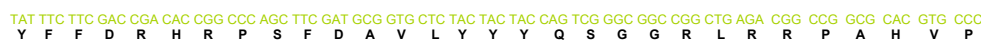

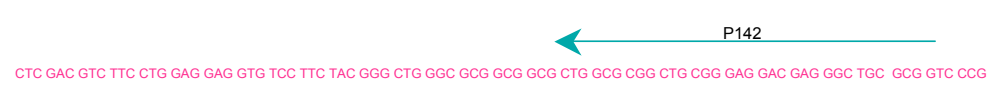
CTC GAC GTC TTC CTG GAG GAG GTG TCC TTC TAC GGG CTG GGC GCG GCG GCG CTG GCG CGG CTG CGG GAG GAC GAG GGC TGC GCG GT

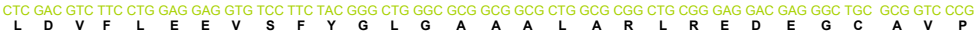

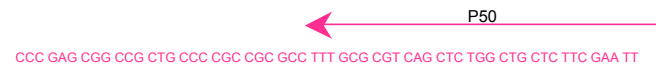

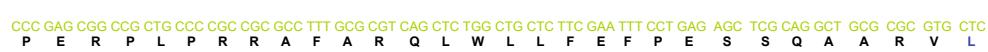

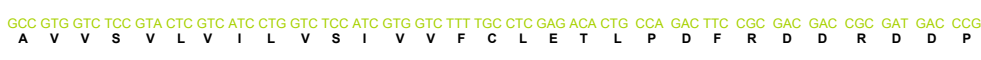
$<$ P38

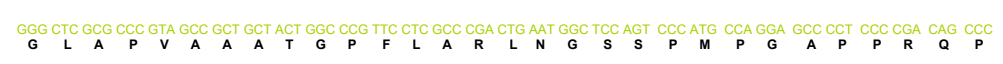
TTC AAC GAT CCA TTC

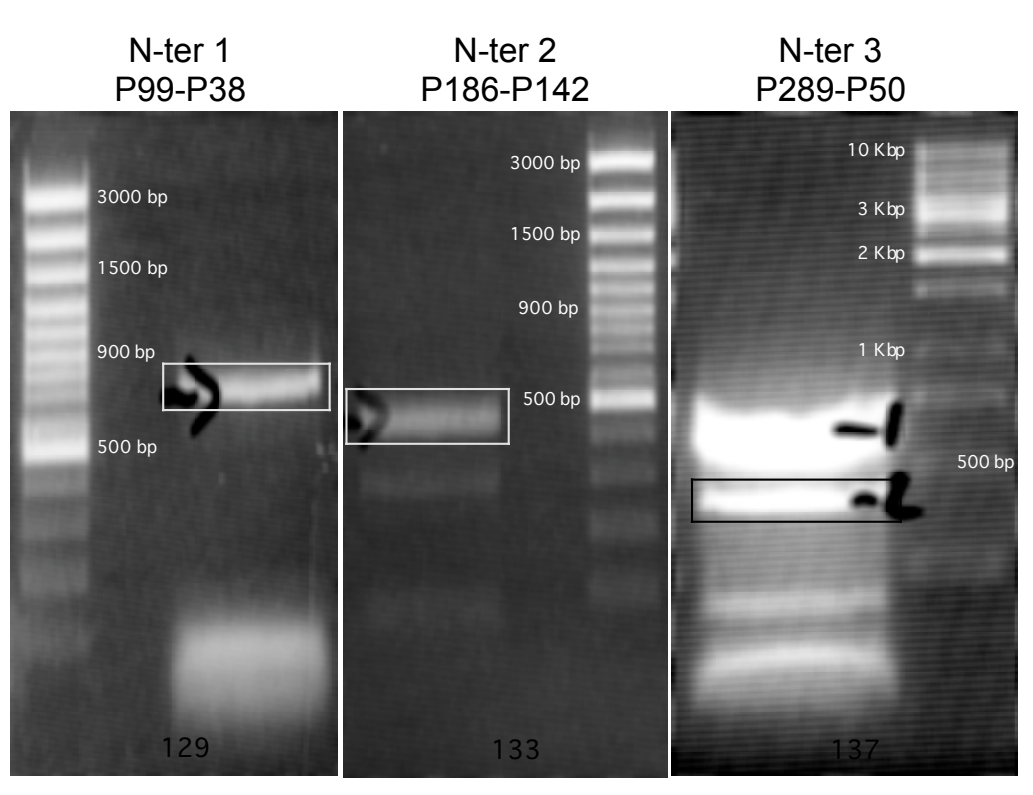

Figure 14. 5' region of the kcna7 gene. Alignment of the three fragments amplified with it's corresponding primer pairs. Translation initiation codons (ATG) are underlined. B. Agarose gel electrophoresis of PCR products from primer pairs P99-P38, $\mathrm{P} 186-\mathrm{P} 142$, and P289-P50 (respectively green, turquoise and pink in A). 
skeletal muscle polyA ${ }^{+}$-RNA. Figure 14 shows the picture of three different gels in which three sets of primer pairs were used to obtain overlapping cDNA fragments from the $5^{\prime}$ end of the kcna7 gene. A fragment of 670bp was obtained with primers

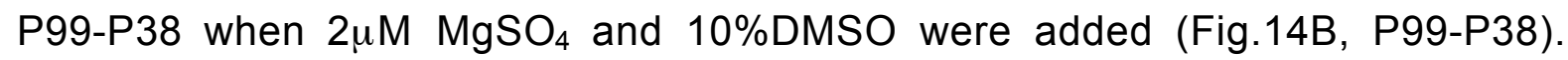
Likewise, primers P186-P142 where useful in the amplification of a fragment of $447 \mathrm{bp}$ after supplementation of the RT-PCR reaction mix with 2-2.4 $\square \mathrm{M} \mathrm{MgSO}_{4}$ and 10\%DMSO (Fig.14B, P186-P142). Finally, we were able to amplify a 634bp fragment with primers P289-P50 in the presence of $2 \square \mathrm{M} \mathrm{MgSO}$ and 10\% DMSO (Fig.14B P289-P50). For all the reactions 5\% DMSO was assayed without good results proving that the secondary structure of the RNA was very strong and therefore only possible to destabilize under very harsh conditions. All the three different products were sequenced completely and resulted identical among themselves and consistent with the EST, Kashuba, and Corfield sequences. Unexpectedly, we observed the occurrence in the same ORF of a first initiation codon "ATG" at position bp 280 that generates the longest translatable sequence. This ORF starts $32 \mathrm{bp}$ downstream from a "stop codon" in frame therefore we estimate that the resulting protein must have 489 amino acids in length (Fig.11) in contrast to the 456 amino acids reported for the human Kv1.7 protein and later for the corrected sequence of the mouse (Kashuba and Bardien, respectively). Due to the differences in the predicted ORF length from the previous reports and our results, we searched for signals important for the early steps of translation, thus we looked for Kozak sequences "AxxATGG". The most highly conserved position within this consensus sequence is the purine base at position -3 that usually is an Adenine (Kozak, 1999). This first ATG lacks the consensus Kozak sequence however functional data shown in the electrophysiological characterization of this work strongly suggest that this fragment of 32 amino acids is functionally relevant hence we have evidence that this part of the protein should be translated (see Functional characterization). Puzzling enough the second ATG does contain a strong Kozak sequence. In order to clarify the translation initiation point further we generated a "mutant" channel that only included the shorter ORF, which is a corresponding sequence to the human channel Kv1.7 that is forced to start the translation at the second ATG.

Additionally the predicted protein from our cDNA clone has two different amino acids in comparison to the sequence reported by Bardien et al. in 2002 (Bardien et 
al., 2002). We observe a leucine in position 117 instead of a valine (equivalent position 84), and a serine in position 468 in replacement of a proline (436 in the Bardien sequence).

\subsection{Cloning of KCNA7}

With the precedent experience of the cloning of the murine cDNA, "One step" RT-PCR was performed using human heart total RNA (Clontech) as template. The cloning strategy consisted in the individual amplification of four fragments corresponding to hfrag1, hfrag-2, hfrag3, and hfrag4 (Fig.15 includes the location of the primers used). hfrag 3 and 4 were successfully amplified with primers P222$\mathrm{P} 223$, and P272-273, respectively using the standard conditions suggested by the manufacturer (Fig.16C and D). Primers P270-P271 were used to amplify hfrag-2 but the PCR reaction resulted successful only upon the supplementation of the reaction mix with $2 \mathrm{mM} \mathrm{MgSO}_{4}$, plus addition of pure DMSO to a final concentration of $10 \%$ (v/v) (Fig.16B). Accordingly, fragment 1 could be amplified with primers 224-225, 10\% DMSO and 2.4\M MgCl 4 (Fig.16A).

The sequence of the human cDNA clone that we amplified corresponded almost identically (>99\%) to the revised sequence NM031886. It is to be noticed that in the last revised sequence from the GeneBank (Accession No. NM031866) the occurrence of "variants" of the KCNA7 gene corresponds to the changes that we observed in our clone. Our human "wild type" sequence contains the single nucleotide polymorfisms (SNPs) resulting in amino acid changes $\mathrm{Y} 71 \mathrm{H}, \mathrm{A} 180 \mathrm{~V}$, and P189R that have been reported as variants in the public database. Additionally in some of the clones other SNPs reported in the GeneBank were found. Table number 1 summarizes the changes we observed in the sequence, the frequency with which they were found and the corresponding SNP identification number. 
GCAGAAGGACGGAGAGAAAGGGGACCCCGGGACGGGAAAGGCGCAGAGCAGGCGCGGGCGGCGGCGGCGGCGGGGCAGGGCAGGGCGGGCGTCCCGGCAGA -222 GGGCGCGCGGTCGCCCTGTCGCCCTCCGCCCCGCCGGGTCACAGTGCCCCCTCCCTCGCGCCCTAGCCGCCCTGCCGGGCTATTTTTACGCGCGGACACC -122

$\mathrm{P} 224 \rightarrow$

GGACACCGGACACCGGGCTGGGGCGGCGGCGGCGGCGGCCGAGGCGGCCGAGGCGGGGCCGCACCGGGGCCGGGCGTCGGGGCCACACGTCGGTTCGCGG -22

GTCGCCGGGGCTGCGCGCGCCCATGGAGCCGCGGTGCCCGCCGCCGTGCGGCTGCTGCGAGCGGCTGGTGCTCAACGTGGCCGGGCTGCGCTTCGAGACGC 079

$\begin{array}{lllllllllllllllllllllllllll}M & E & P & R & C & P & P & P & C & G & C & C & E & R & L & V & L & N & V & A & G & L & R & F & E & T & 026\end{array}$

GGGCGCGCACGCTGGGCCGCTTCCCGGACACTCTGCTAGGGGACCCAGCGCGCCGCGGCCGCTTCTACGACGACGCGCGCCGCGAGTATTTCTTCGACCG 179

$\begin{array}{lllllllllllllllllllllllllllllllllll}R & A & R & T & L & G & R & F & P & D & T & L & L & G & D & P & A & R & R & G & R & F & Y & D & D & A & R & R & E & Y & F & F & D & R & 060\end{array}$

GCACCGGCCCAGCTTCGACGCCGTGCTCTACCACTACCAGTCCGGCGGGCGGCTGCGGCGGCCGGCGCACGTGCCGCTCGACGTCTTCCTGGAAGAGGTG 279

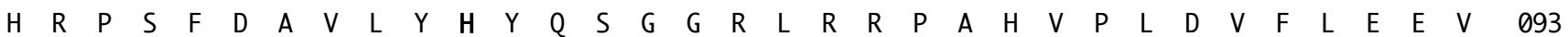
$\mathrm{P} 270 \longrightarrow$

GCCTTCTACGGGCTGGGCGCGGCGGCCCTGGCACGCCTGCGCGAGGACGAGGGCTGCCCGGTGCCGCCCGAGCGCCCCCTGCCCCGCCGCGCCTTCGCCC 379

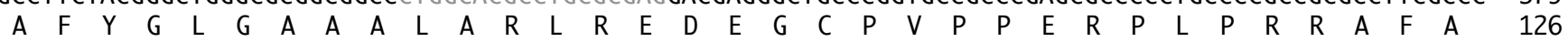
$\leftarrow \quad \mathrm{P} 225$

GCCAGCTGTGGCTGCTTTTCGAGTTTCCCGAGAGCTCTCAGGCCGCGCGCGTGCTCGCCGTAGTCTCCGTGCTGGTCATCCTCGTCTCCATCGTCGTCTT 479

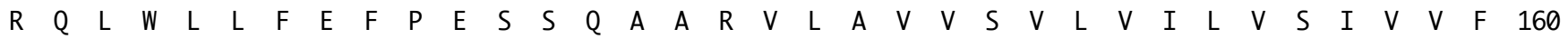
P222

CTGCCTCGAGACGCTGCCTGACTTCCGCGACGACCGCGACGGCACGGGGCTTGCTGCTGTAGCCGCAGCCGGCCCGTTCCCCGCTCGGCTGAATGGCTCC 579

$\begin{array}{lllllllllllllllllllllllllllllllllll}C & L & E & T & L & P & D & F & R & D & D & R & D & G & T & G & L & A & A & V & A & A & A & G & P & F & P & A & R & L & N & G & S & 193\end{array}$ $\leftarrow \quad \mathrm{P} 271$

AGCCAAATGCCTGGAAATCCACCCCGCCTGCCCTTCAATGACCCGTTCTTCGTGGTGGAGACGCTGTGTATTTGTTGGTTCTCCTTTGAGCTGCTGGTAC 679 $\begin{array}{lllllllllllllllllllllllllllllllllll}S & Q & M & P & G & N & P & P & R & L & P & F & N & D & P & F & F & V & V & E & T & L & C & I & C & W & F & S & F & E & L & L & V & 226\end{array}$ GCCTCCTGGTCTGTCCAAGCAAGGCTATCTTCTTCAAGAACGTGATGAACCTCATCGATTTTGGTGGCTATCCTTCCCTACTTTGTGGCACTGGGCACCGA 0779 $\begin{array}{llllllllllllllllllllllllllllllllllll}R & L & L & V & C & P & S & K & A & I & F & F & K & N & V & M & N & L & I & D & F & V & A & I & L & P & Y & F & V & A & L & G & T & E & 0260\end{array}$ GCTGGCCCGGCAGCGAGGGGTGGGCCAGCAGGCCATGTCACTGGCCATCCTGAGAGTCATCCGATTGGTGCGTGTCTTCCGCATCTTCAAGCTGTCCCGG 0879

$\begin{array}{lllllllllllllllllllllllllllllllllll}L & A & R & Q & R & G & V & G & Q & Q & A & M & S & L & A & I & L & R & V & I & R & L & V & R & V & F & R & I & F & K & L & S & R & 0293\end{array}$ $<\mathrm{P} 223$

CACTCAAAGGGCCTGCAAATCTTGGGCCAGACGCTTCGGGCCTCCATGCGTGAGCTGGGCCTCCTCATCTTTTTCCTCTTCATCGGTGTGGTCCTCTTTT 0979 $\begin{array}{llllllllllllllllllllllllllllllllllll}H & S & K & G & L & Q & I & L & G & Q & T & L & R & A & S & M & R & E & L & G & L & L & I & F & F & L & F & I & G & V & V & L & F & 0326\end{array}$ $\mathrm{P} 223$

CCAGCGCCGTCTACTTTGCCGAAGTTGACCGGGTGGACTCCCATTTCACTAGCATCCCTGAGTCCTTCTGGTGGGCGGTAGTCACCATGACTACAGTTGG 1179 $\begin{array}{llllllllllllllllllllllllllllllllllll}S & S & A & V & Y & F & A & E & V & D & R & V & D & S & H & F & T & S & I & P & E & S & F & W & W & A & V & V & T & M & T & T & V & G & 0360\end{array}$ CTATGGAGACATGGCACCCGTCACTGTGGGTGGCAAGATAGTGGGCTCTCTGTGTGCCATTGCGGGCGTGCTGACTATTTCCCTGCCAGTGCCCGTCATT 1279

$\begin{array}{lllllllllllllllllllllllllllllllllll}Y & G & D & M & A & P & V & T & V & G & G & K & I & V & G & S & L & C & A & I & A & G & V & L & T & I & S & L & P & V & P & V & I & 0393\end{array}$ GTCTCCAATTTCAGCTACTTTTATCACCGGGAGACAGAGGGCGAAGAGGCTGGGATGTTCAGCCATGTGGACACGCAGCCTTGTGGCCCACTGGAGGGCA 1379

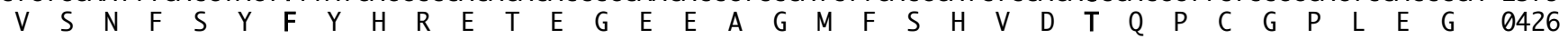
$\leftarrow \quad P 273$

AGGCCAATGGGGGGCTGGTGGACGGGGAGGTACCTGAGCTACCACCTCСACTCTGGGCACCCCCAGGGAAACACCTGGTCACCGAAGTGTGAGGAACAGT 1479

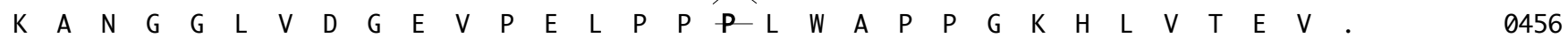
$<P 273$

TGAGGTCTGCAGAATCACTAGTGAATTCGCGGCCGCCTGCAGGTCGACCTCGAGGGGGGCCCCAGCTATCTGG

Figure 15. cDNA and amino acid sequences of KCNA7. The primers used are indicated with the arrows and corresponding light gray font. Translation initiation codon ATG is underlined. The Kozac consensus sequence marked with the position numbers. SNPs are marked in bold. 


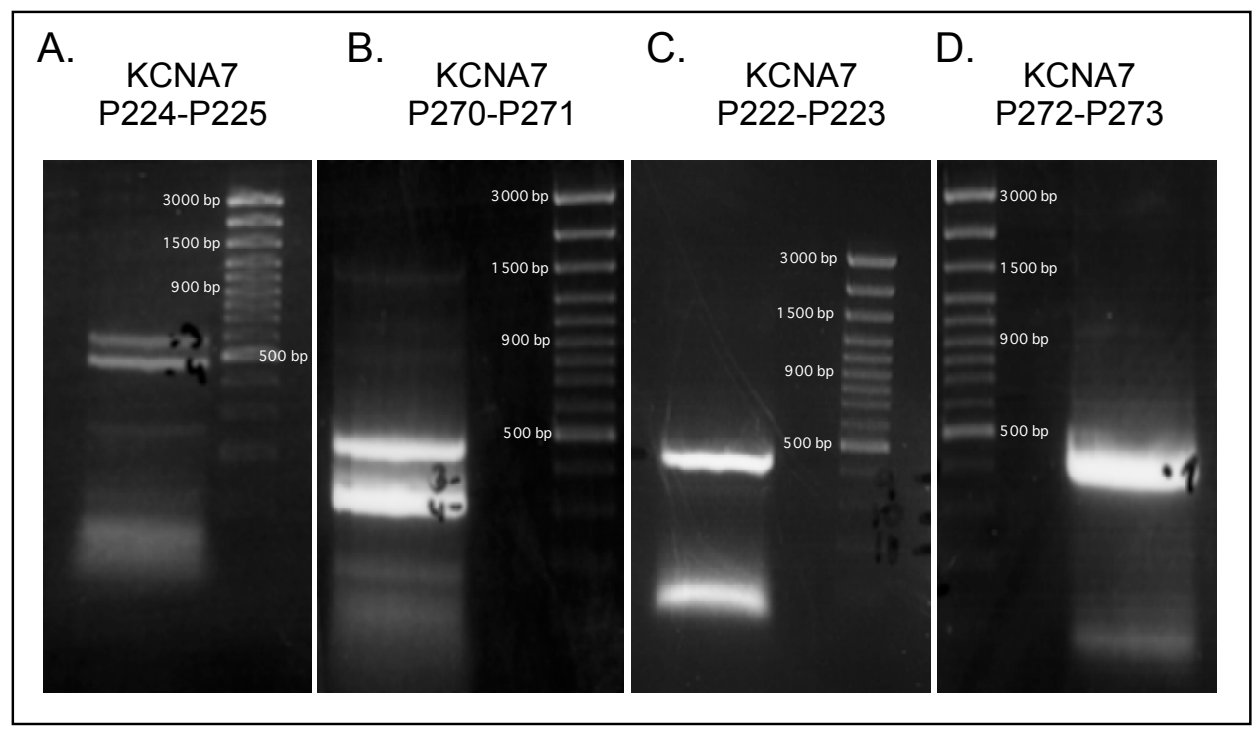

Figure 16. Agarose gel electrophoresis of the One step RT-PCR products corresponding to the human KCNA7 cDNA products. From $A$ to $D$ the amplification products from the most 5 ' terminal fragment to the 3 ' end.

Table 1.

Single Nucleotide Polymorphisms

\begin{tabular}{|c|c|c|c|c|c|}
\hline DbSNP & $\begin{array}{l}\text { Codon } \\
\text { Position }\end{array}$ & Translation & Occurrence & $\begin{array}{c}\text { Frequency } \\
\%\end{array}$ & Ref \\
\hline & & Kashuba et al., 2001 & $1 / 19$ & 5.3 & NM_031866 \\
\hline $\mathrm{T} / \mathrm{C}$ & 1 & $\mathrm{Y} 71 \mathrm{H}$ & $19 / 19$ & 100 & the present work \\
\hline $\mathrm{T} / \mathrm{C}$ & 2 & A180V & $19 / 19$ & 100 & the present work \\
\hline \multirow[t]{2}{*}{$\mathrm{G} / \mathrm{C}$} & \multirow[t]{2}{*}{2} & P189R & $19 / 19$ & 100 & rs1611775 \& our \\
\hline & & $\begin{array}{c}Y 71 \mathrm{H}+\mathrm{A} 180 \mathrm{~V}+ \\
\mathrm{P} 189 \mathrm{R}\end{array}$ & $19 / 19$ & 100 & the present work \\
\hline $\mathrm{C} / \mathrm{T}$ & 1 & S340P & $13 / 19$ & 68.4 & the present work \\
\hline $\mathrm{C} / \mathrm{T}$ & 2 & F400S & $19 / 19$ & 100 & the present work \\
\hline $\mathrm{T} / \mathrm{C}+\mathrm{CCT} /--$ & 2 & $\mathrm{M} 418 \mathrm{~T}+\Delta 441$ & $13 / 19$ & 10.6 & rs1017219 \\
\hline \multirow[t]{2}{*}{-} & & & & & rs3840913 \\
\hline & & Wild type & $1 / 19$ & 5.3 & NM_031866 \\
\hline
\end{tabular}




\subsection{Promoter analysis}

The nucleotide sequence of the 5 ' end of the mouse clone was analyzed further with the Dragon Promoter Finder tool (Singapore, Bajic et al., 2003) and the GenomatixSuite promoter analysis software (Genomatix Software $\mathrm{GmbH}$, München, Germany). The most $5^{\prime}$ terminal fragment includes a stretch of $\sim 150$ nucleotides in the mouse sequence and $\sim 250$ for the human, upstream of the OFR of the kcna7/KCNA7 sequence cloned in this work.

Mouse kcna7 promoter

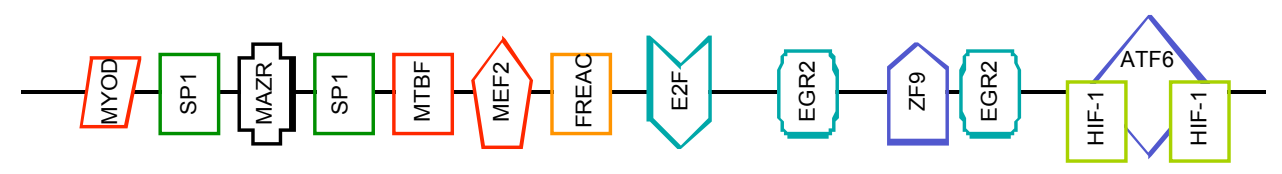

Human KCNA7 promoter

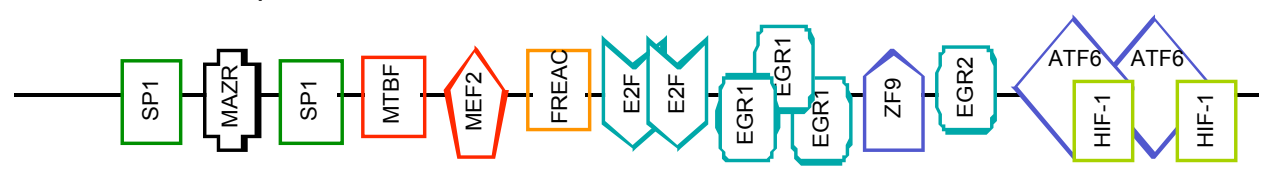

Figure 17. Putative promoter sequence organization and transcription factor binding sites. Red framed boxes belong to the myogenic cluster. The stress related TFs are in dark blue and green. All the other colors are cell cycle related TFs.

The identified putative regulatory regions resulted congruent between themselves, that is both potential promoters, corresponding to the mouse and human genes cloned in this work. Several transcription factor binding site sequences (TFs) are present in a $164 \mathrm{bp}$ stretch of the 5 ' end. Figure 17 shows a comparison of the putative mouse and human promoters. The polymerase II dependent promotor sequences were identified using the PromoterInspector software (Genomatix Software $\mathrm{GmbH}$, Germany) and the TF determination was performed with the help of the GEMS Launcher (Genomatix Software GmbH, Germany). As it can be observed the similarity in the organization of both promoter regions is evident. It is remarkable that the organization between the mouse and human putative promoter regions is very similar. At first glance, the TFs can be distinguished into three clusters of binding sites: 1) "myogenic", including mostly muscle-specific transcriptional activators; 2) "cell cycle related" and 3) "stress" related clusters of transcription factor binding sites. In the myogenic cluster of mouse are found the TFs of: MYOD, MTBF 
and MEF2. The cell cycle related TFs cluster includes FREAC2, SP1, E2F, EGR2 and 3 and ZF9. And the stress related cluster includes ATF6 and HIF1.

\subsection{Protein analysis}

The proteins encoded by the kcna7 and KCNA7 cDNA clones share amino acid sequence conservation over 93\%, therefore they are regarded as orthologous (Fig.18). The predicted Kv1.7 wt protein is composed of 489 amino acids with an estimated molecular weigh of $\sim 54 \mathrm{kDa}$, isolelectric point of 7.98 and a total charge of 4.13 at pH 7. Accordingly the estimated molecular weighs of mKv1.7-T0 (457aa) and hKv1.7 (456aa) are about 51kDa; the isoelectric points are 7.54 and 7.56 for the mouse mutant and the human protein, and the net charge 1.8 and 1.96 (at $\mathrm{pH} 7$ ) respectively. Observe that the presence of the $\mathrm{N}$-terminal 32 amino acids present in the mKv1.7 wt protein has a strong effect on the net charge of the protein adding approximately 2 charges that are concentrated in the $\mathrm{NH}_{2}$ domain. Analysis of the protein sequences confirmed the identity of $\mathrm{Kv}$ channels with all the structural hallmarks of potassium selective voltage gated related ion channels (Fig.18).

The potential transmembrane segments were detected with the help of the PSORT software (Nakai \& Horton, 1999) that employs the method developed by Klein et al.'s (Klein et al, 1985; modified by Nakai \& Kanehisa, 1992). It identifies the most probable transmembrane segment from the average Kyte-Doolittle hydrophobicity value over a window of 17-residue segments (minimum length of an $\square$-helical membrane spanning segment). Figure 19 depicts the hydrophobicity plot of mouse (19A) and human (19B) Kv1.7 proteins. In figure 20 six hydrophobic membrane-spanning segments are clearly distinguishable and designated according to the standard nomenclature S1 to S6 (Kyte \& Doolittle, 1982). Between S5 and S6 is the "signature sequence" that includes amino acids TVGYG. The GXG motif is present within the pore of all known potassium selective channels. S4 is believed to form an important part of the voltage sensor of $\mathrm{Kv}$ channels since it contains a positive charge every 3rd amino acids $\left({ }^{+} X X^{+} X X^{+} X X^{+}\right.$, mostly arginines) (Noda et al., 1984). This feature has been positively correlated with voltage sensitivity of the channel. 


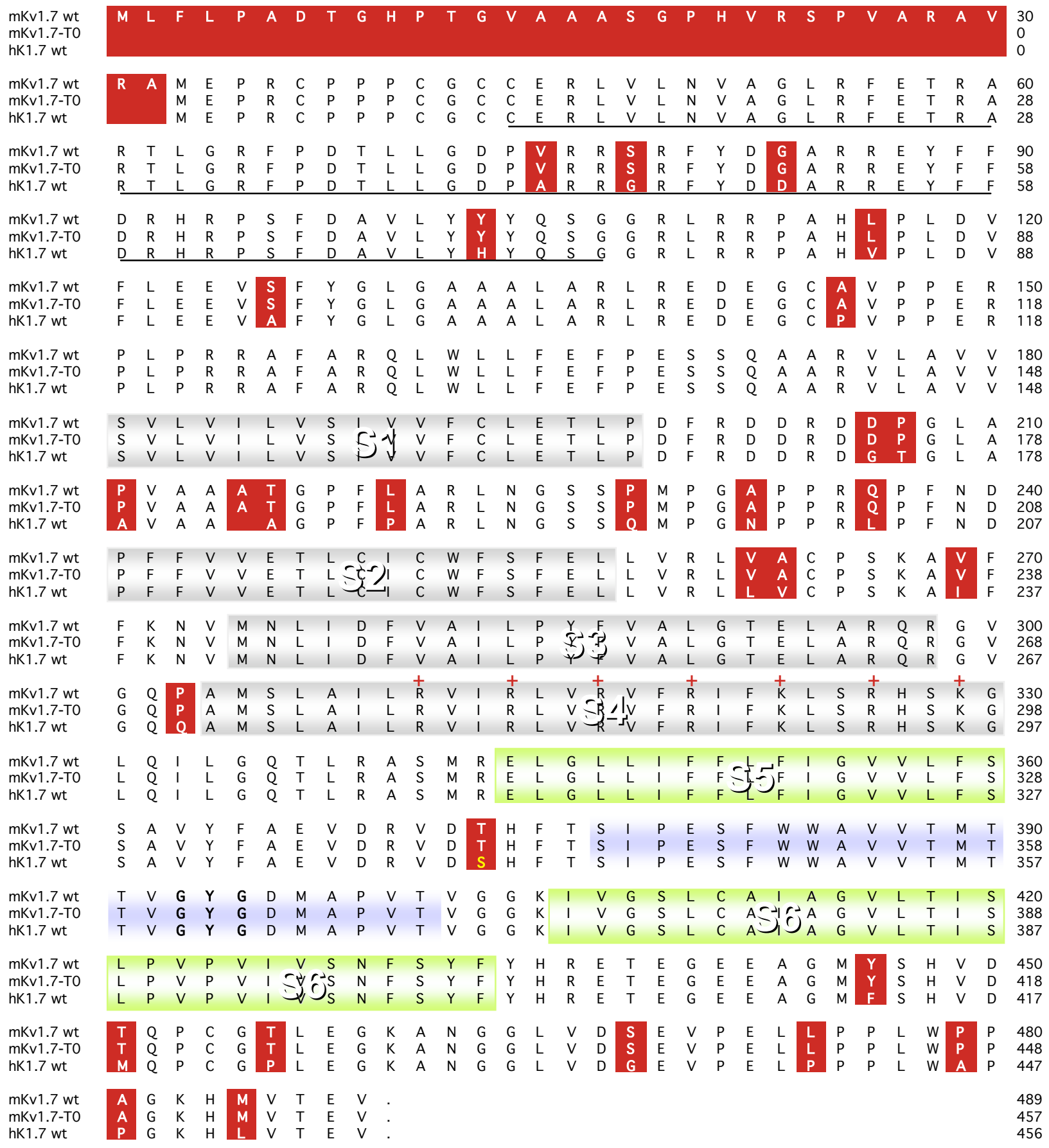

Figure 18. Alignment of the Kv1.7 channels. The amino acid sequence of the mouse Kv1.7 wt, mKv1.7-T0 and hKv1.7 wt. N-terminal region and sequence disagreements are highlighted in red. The T1 domain is underlined. The voltage sensor region segments (S1-S4) are in gray shaded boxes. The pore region S5-S6 is in green shaded boxes. The H5 (S5-S6 linker) is shaded and the signature sequence GYG is in bold. + Positive charges of S4. 


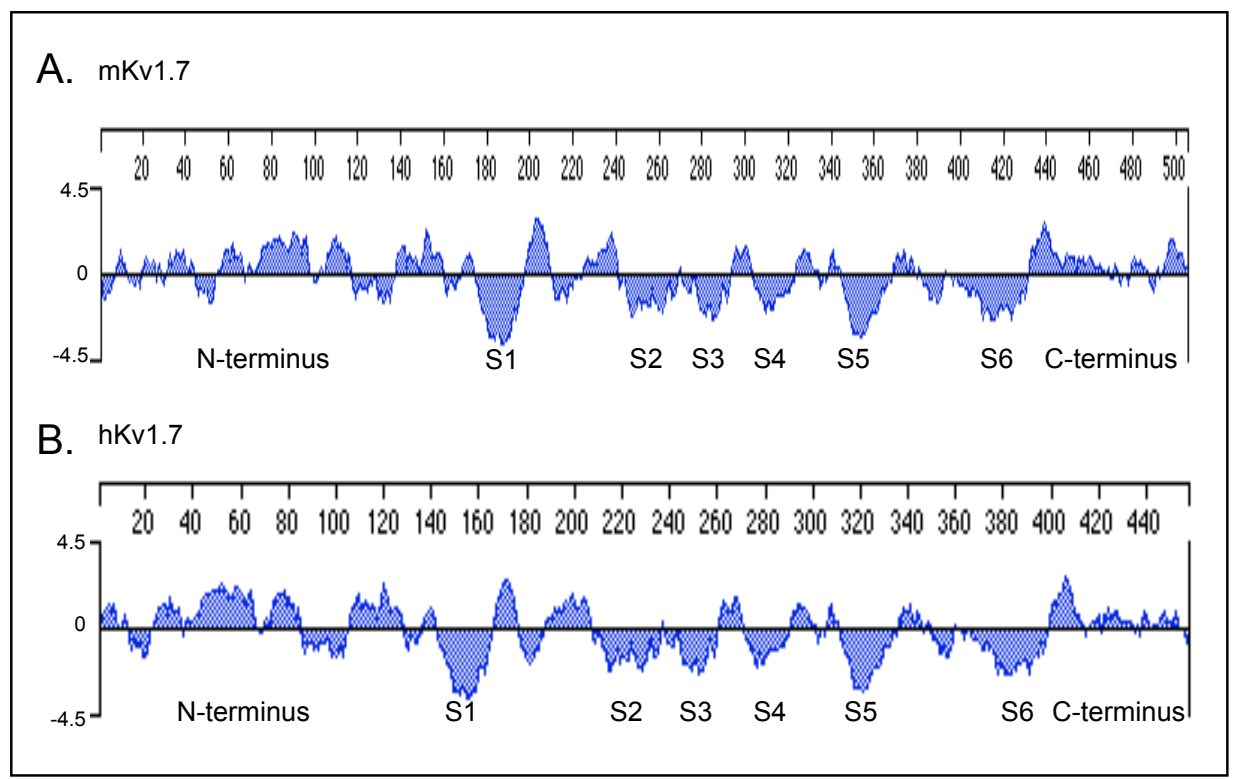

Figure 19. Kyte-Doolitle hydrophobicity plots. A, mouse Kv1.7 channel; B, human Kv1.7 channel.

The proteins were next analyzed according to the prediction of membrane topology method developed by Hartmann et al. (Hartmann et al., 1989). This method assumes that the overall topology of eukaryotic membrane proteins is determined by the net charge difference of 15 residues flanking the most $\mathrm{N}$-terminal transmembrane segment on both sides. According to this analysis the $\mathrm{N}$-terminal region of the protein is believed to reside at the cytoplasmic side of the membrane. In the cytoplasmic Nterminus a stretch of 100 amino acids shares $70 \%$ homology with the tetramerization domain motif observed in members of the Kv subfamily. The $\mathrm{N}$-terminal, cytoplasmic tetramerization domain (T1) of voltage-gated $\mathrm{K}^{+}$channels encodes molecular determinants for subfamily-specific assembly of alpha-subunits into functional tetrameric channels and it is a member of the BTB/POZ domain (pfam00651) (poxvirus and zinc finger/broad-complex, tramtrack and bric a' brac, the genes where the domain homology was originally identified) one of the largest families of protein-protein interaction domains (Zollman et al., 1994; Choe et al., 1999).

Furthermore, there is a putative $\mathrm{N}$-linked glycosylation site in the extracellular loop between S1 and S2 that is conserved within mouse, human and Drosophila (positions 224, 191 and 263, respectively). There is a putative consensus PKC 
QLQRNSLDGYGSLPKLSSQDEEGGAGHGFGGGPQHFEPIPHDHDFCERVV 100 hKv1.7 - MLFLPADTGHPTGVAAASGPHVRSPVARAVRAMEPRCPPPCGCCERLV 48

Shaker

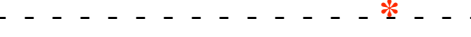
mKv1.7 LN VA G LRFETRARTLGRFP D T L G D P VRRSRF Y D GARREYFFDRHRP $S F D 98$

IN VSGLRFETQLRTLNQFPDTLLGDPARRLRYFDPLRNEYFFDRSRPSFD 150 hKV1.7 LNVAGLRFETRARTLGRFPDTLLGDPARRGRFYDDARREYFFDRHRPSFD 66

Shaker A ILYYYQSGGRLRRPVNVPLDVFSEEIKFYELGDQAINKFREDEGFIKEE 200 mKv1.7 AVLYYYQSGGRLRRPAHLPLDVFLEEVSFYGL GैA A L ARLREDEGCAVPP 148 hKv1.7 Shaker mKv1.7 A VLYHYQSGGRLRRPAHVPLDVFLEEVAFYGLGAAALARLREDEGCPVPP 116 S1

hKv1.7

ERPLPDNEKQRKVWLLFEYPESSQAARVVAIISVFVILLSIVIFCLETLP 250 ERPLPRRAFARQLWLLFEFPESSQAARVLAVVSVLVILVSIVVFCLETLP 198 ERPLPRRAFARQLWLLFEFPESSQAARVLAVVSVLVILVSIVVFCLETLP 166

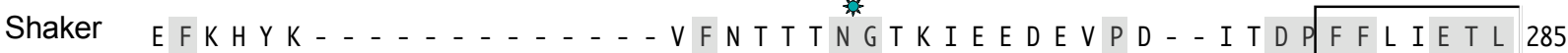
mKV1.7 DFRDDRDDPGLAPVAAATGPFLARLNGSSPMPGAPPRQPFNDPFFVVETL 248 hKv1.7 DFRDDRDGTGLA A VAAA-GPFPARLNGSSQMPGNPPRLPFNDPFFVVETL 215 Shaker C I I W F T FE L TVRFLACPNKLN F C R D V M N V I D I I A I I P Y F I T L A T V V A E E E 335 mKV1.7 C I C W F S F E L L VRLVACPS K A V F F K N V M N L I D F V A I L P Y F V A L G*T E L A R Q R 298 hKV1.7 C I C W F S FELLVRLLVC P S K A I F F K N VMNLI D F VA I L P Y F V A L G T E L A R Q R 265

Shaker DTLNLPKAPVSPQDKS SNQAMSLA ML R I R L V R VF R IFKLS RHSKGLQIL 385 mKv1.7 GV ............ G Q P A M S L A I LRVIRLVRVFRIFKLSRHSKGLQIL 334

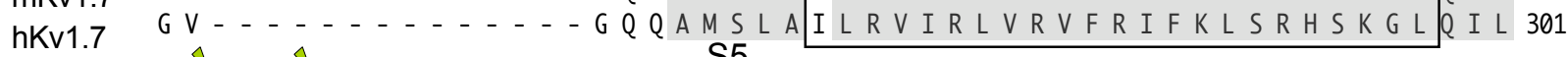

Shaker

mKv1.7

$\mathrm{hK} v 1.7$

GRTLKASMRELGLLIFFLFIGVVLFSSAVYFAEAGSENSFFKSIPDAFWW 435 S5 GQTLRASMRELGLLIFFLFIGVVLFS SAVYFAEVDRVDTHFTS IPESFWW 384 GQTLRASMRELGLLIFFLFIGVVLFS SAVYFAEVDRVDSHFT:SIPESFWW 351 S6

Shaker A V V TMTTVGYGDMTPVGVWGKIVGSLCA I A GVLTIALPVPVIVSNFNYFY 485

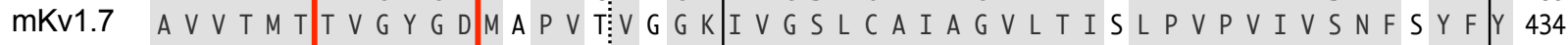
hKV1.7 AVVTMTTVGYGDMAPVTVGG KIVGSLCA I A G VL T I S L P V P V I V S N F S Y F Y 401

Shaker HRETDQEEMQSQNFNHVTSCPYLPG̈TLVGQHMKKSSLSESSSDMMDLDDG 535 mKV1.7 HRETEGEEAGMYSHVDTQPCGTLEGKANGGLVDPEVPELLPPLWPPAGKH 484 hKV1.7 HRETEGEEAGMFSHVDMQPCGPLEGKANGGLVDGEVPELPPPLWAPPGKH 451

Shaker VESTPGLTETHPGRSAVAPFLGAQQQQQQQPVASSLSMSIDKQLQHPLQQ585 mKV1.7 M V T E V.

hKV1.7 L V T E V .

Shaker LTQTQLYQQQQQQQQQQQNGFKQQQQQTQQQLQQQQSHTINASAAAATS 635

Figure 20. Alignment of the Kv1.7 and Shaker. The amino acid sequence of mouse and human Kv1.7 channels are compared to the Drosophila Shaker channel. Conserved amino acids are in light gray. Transmembrane spanning segments (S1-S6) are within boxes. The T1 domain is highlighted with striped background, underlined the deleted segment for the mKv1.7- $\square \mathrm{T} 1$. The pore region has a doted line box and the signature sequence is within the red frame. S1-S4 form the voltage sensor and S5-S6 form the pore domain. Positive charges of S4 are marked with red plus signs. Indicates a putative Tyrosine kinase phosphorilation site. N-glycosylation site. shows the PKC sites. The red asterisk * indicates putative myristoylation sites. Alignment was made with ClustalW followed by manual adjustment and exclusion of loops. Shaker Drosophila melanogaster (AccessionNo. P08510). 
phosphorylation site in the cytoplasmic loop between S4 and S5 in mouse, human, and Drosophila (positions 337, 304, and 388, respectively). Additionally, there is a conserved putative tyrosine phosphorylation site in the N-terminus (T1 domain) of mouse, human and Drosophila (positions 94, 62, and 146, respectively). Finally several myristoylation signals are present within the whole protein (Fig.20). 


\subsection{Functional characterization of Kv1.7 channels}

\subsection{1 mKv1.7}

The nucleotide sequence analysis of the mKv.7 wt cDNA indicated (kcna7), to our surprise, the presence of an AUG (initiation of transcription codon) in frame $\sim 100$ nucleotides before the AUG reported as the start of transcription of Kv1.7 channels (Kashuba et al., 2001; Bardien et al., 2002). The presence of 32 more amino acids in the $\mathrm{N}$-terminal region of our construct suggested the possibility of kinetic differences between our wild type mouse Kv1.7 channels and those reported in the literature. To clarify if those 32 amino acids were indeed transcribed and if they could have a functional relevance on the channel behavior, the mKv1.7-T0 mutant construct was generated from which the whole 100 nucleotides fragment bearing the first AUG in frame were deleted and the electrophysiological characteristics of both constructs investigated.

The Xenopus laevis oocytes heterologous expression system was used to study mouse Kv1.7 wild type (mKv1.7 wt), and two N-terminal mKv1.7 mutants that included mKv1.7-T0 ( $\Delta 1-32)$, the tetramerization domain (T1) mutant mKv1.7 $\Delta T 1$ ( $\Delta 34-72)$; as well as the human wild type Kv1.7 (hKv1.7 wt) channels. Oocytes from stages $\mathrm{V}$ and $\mathrm{VI}$ were injected with the corresponding cRNAs and two electrodes voltage clamp (TEVC) as well as patch clamp experiments were carried out in order to perform the electrophysiological characterization of Kv1.7 channels.

The amino acidic sequence of Kv channels includes two intracellular domains comprised by the amino and carboxy termini of the protein, and six hydrophobic segments that are believed to span the plasma membrane and have been related to serve defined functions within the protein (Fig.18; Fig.20). A fragment of about 100 amino acids in the $\mathrm{N}$-terminal region, the tetramerization domain T1 of Kv channels, has been reported to constitute the coordinating domain for the tetrameric assembly of functional channels through the presence of a POZ/BTB protein-protein interaction region (Zollman et al., 1994; Choe et al., 1999). 
A.

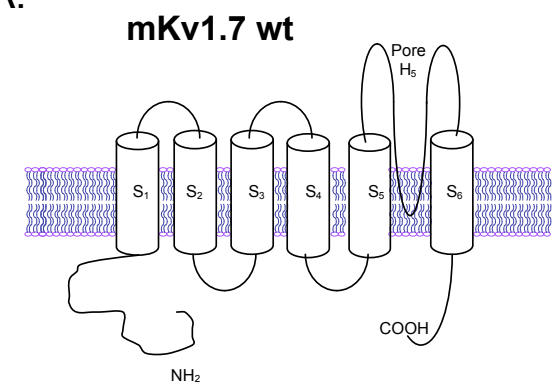

C.

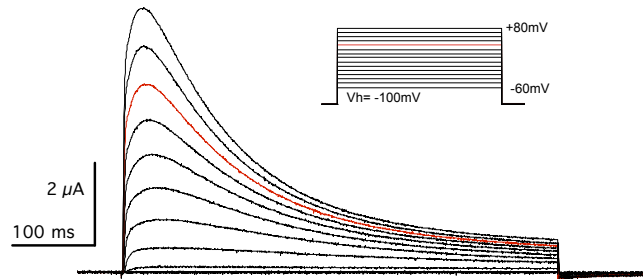

E.

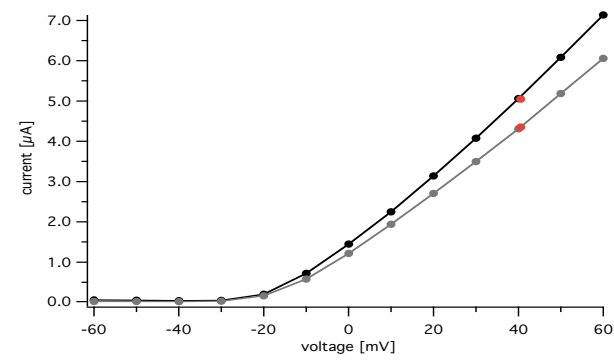

B.

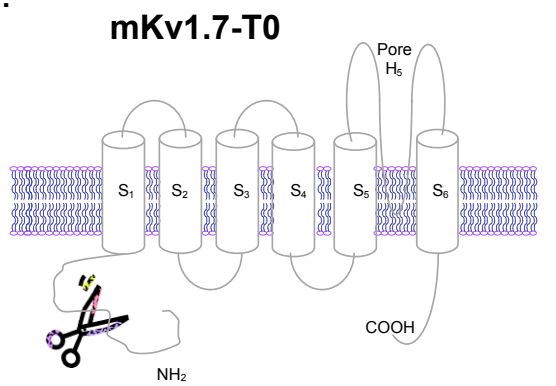

D.

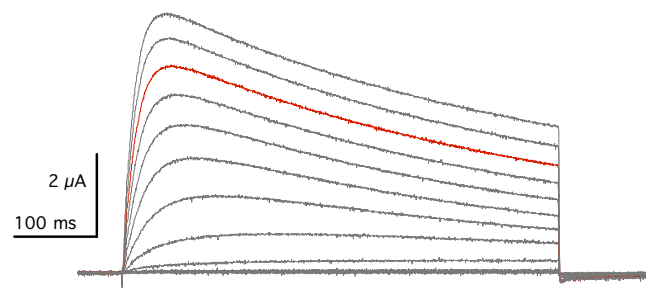

$\mathrm{F}$.

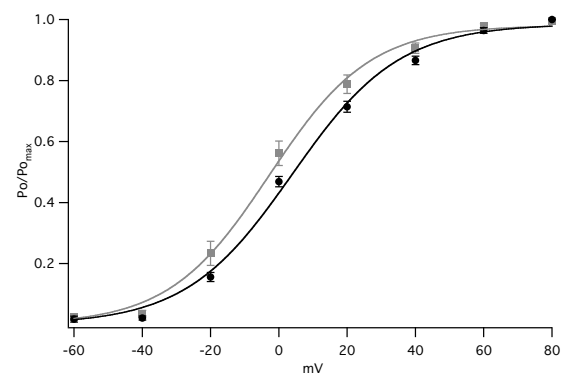

Figure 21. Representative currents from $X$. laevis oocytes expressing mKv1.7 wt and mKv1.7-T0 channels. A. and B. Schematic representation of the mKv1.7 wt and mKv1.7-T0 constructs. C. and D. Outward currents in response to $500 \mathrm{~ms}$ depolarizing test potentials ranging from -60 to $+80 \mathrm{mV}$ in $10 \mathrm{mV}$ increments $(15 \mathrm{secec}$ between pulses), $V_{h}=-100 \mathrm{mV}$. Mouse Kv1.7 wt is shown in black (C.) and mKv1.7T0 in gray (D.) the red trace is current at $+40 \mathrm{mV}$. E. Current voltage relationships (IV) from the experiments shown in A and B. Peak currents are plotted as a function of membrane potential $\left(V_{m}\right)$. F. Steady state activation curves for mKv1.7 wt $(n=20)$ and mKv1.7-T0 $(n=15)$ were constructed from the normalized macroscopic conductance. Mean \pm sem relative open probabilities were plotted against the potential and were fitted to a Boltzmann function (see methods).

In order to verify the importance of the T1 domain (Fig18, Fig.20) of mKv1.7 we generated the mKv1.7- $\mathrm{TT} 1$ consisting of a deletion mutant mKv1.7 missing a fragment that encoded for 42 amino acids from the alleged T1 domain (Fig.7). Oocytes tested during up to 7 days after injection with cRNAs encoding for the T1 domain mutant, mKv1.7 $\Delta \mathrm{T} 1$ failed to elicit voltage dependent currents (distinguishable from leak current of 100 to $400 \mathrm{nA}$ present in the oocytes) upon ramp protocols in the range between -150 to $+150 \mathrm{mV}$. Thus we conclude that the 
resembling T1 region (or at least some of the amino acids in the deleted 42 amino acids stretch) present in mKv1.7, and by homology in hKv1.7 channels, is crucial for functional expression. The relevance of $\mathrm{T} 1$ could be relative to the assembling, trafficking or operation of the channels. Reports on the literature offer vast information on the influences of the T1 region in channel assembly and tetramerization.

The N-termini of $\mathrm{Kv}$ channels have been extensively investigated for its inherence in the development of fast inactivation. Furthermore, segments S1 to S4 are involved in the voltage sensitivity of the channel, more specifically the S4 segment contains positively charged amino acids and has been associated with the steep voltage dependence of the opening and closing, gating, of the permeation pathway of ion channels, thus S4 is regarded as one of the main components of the "voltage sensor". The sequence of the Kv1.7 channels cloned in this work includes a characteristic S4 segment (Fig.18, Fig.20), consequently the gating of this channel was expected to be responsive to changes in the membrane potential and therefore voltage dependent. The voltage dependence of currents through ion channels can be evaluated by determining the influence that different membrane command potentials $\left(V_{m}\right)$ can exert on the currents flowing through ion channel proteins. Voltage steps to different depolarizing potentials were used to characterize the properties of $\mathrm{mKv} 1.7$ wild type and mKv1.7-T0 currents in control conditions, defined by the presence of $2.5 \mathrm{mM} \mathrm{K}^{+}\left(115 \mathrm{mM} \mathrm{Na}{ }^{+}\right.$, Normal Frog Ringer solution, NFR) as the bath solution. Figure 21 shows two families of current traces corresponding to mKv1.7 wt (panel C.) and mKv1.7-T0 (panel D.) channels in response to the "IV" stimulation protocol shown in the inset (IV stands for current-voltage). Cells were kept at a holding potential $\left(V_{h}\right)$ of $-100 \mathrm{mV}$, where the open probability $\left(P_{0}\right)$ for $\mathrm{mKv} 1.7$ channels is expected to be minimal. A series of depolarizing pulses from $-60 \mathrm{mV}$ to $+80 \mathrm{mV}$ with duration of 500 milliseconds were applied, with 15 seconds inter-pulse intervals to allow equilibration of the channels after each pulse. Depolarizing voltage steps in Xenopus oocytes injected with mKv1.7 wt and mKv1.7-T0 cRNAs elicited voltage dependent outward currents with increasingly positive values that started to activate at potentials more positive than $-30 \mathrm{mV}$ (Fig.21C, D, and E.). Currents from mKv1.7 channels activated rapidly (in the millisecond range) and were voltage dependent as 
observed from the IV relationship plots that were constructed with the peak currents (maximal current amplitude during each pulse). The relative open probabilities $\left(P_{\mathrm{o}} / \mathrm{P}_{\text {omax }}\right)$ derived from the initial currents were plotted against the voltages of the depolarizations and fit with a Boltzmann function, $\mathrm{P}_{0} / \mathrm{P}_{\text {omax }}$ or

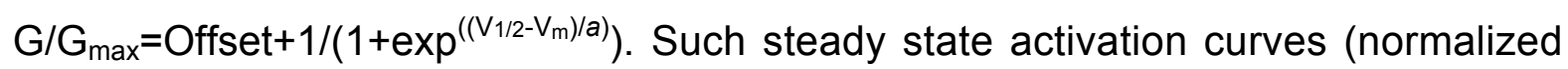
macroscopic conductance, $G / G_{\max }$ versus the potential, $V$ ) are shown in figure $21 \mathrm{~F}$, data points are given as mean \pm sem and the smooth line corresponds to the Boltzmann fit. From such plots the half activation potential $\mathrm{V}_{1 / 2}$, and the slope factor a, were calculated.

Table 2.

Steady state activation parameters of mKv1.7 channels.

\begin{tabular}{cccc}
\hline & $\begin{array}{c}\text { mKv1.7 wt } \\
\text { mean } \pm \text { sem }\end{array}$ & $\begin{array}{c}\text { mKv1.7-T0 } \\
\text { mean } \pm \text { sem }\end{array}$ & $\mathrm{p}$ \\
\hline & & & \\
$\mathrm{V}_{1 / 2}$ & $4.34 \pm 1.36$ & $-4.33 \pm 2.9$ & 0.0068 \\
Slope & $15.4 \pm 0.5$ & $13.3 \pm 0.6$ & 0.0107 \\
\hline $\mathrm{n}$ & 20 & 15 & \\
\hline
\end{tabular}

The murine wild type channel presented half activation potential $\left(\mathrm{V}_{1 / 2}\right)$ of about $5 \mathrm{mV}$ and a voltage dependence of $15 \mathrm{mV}$ per e-fold change in relative $P_{0}$. Surprisingly, the absence of the 32 amino acids in the $\mathrm{N}$-terminal part of mKv1.7 caused a negative shift of the voltage range for activation, the $\mathrm{N}$-terminus deletion mutant mKv1.7-T0 presented a $V_{1 / 2}$ of about $-4 \mathrm{mV}$. Which is slightly but significantly shifted to more hyperpolarized potentials (Table 2, Fig 21D). These results suggest some influence of the $\mathrm{N}$-terminal region on the voltage sensing of this channels. The amino terminal region of $\mathrm{mKv} 1.7$ wt channels has a net charge of 6.45 in comparison to 4.12 from T0 (at pH 7). Consequently, figure 21 confirms that mKv1.7 channels share qualitative and quantitative properties with the voltage dependent potassium channel family as expected from the protein sequence analysis. 


\subsubsection{1 lonic selectivity}

The presence of the Kv channel signature sequence GYG in the pore region of mKv1.7 channels is a robust indication of mKv1.7 being strongly selective for $\mathrm{K}^{+}$ versus other monovalent ions and more specifically to other cations. To examine the selectivity of mKv1.7 channels for $\mathrm{K}^{+}$ions the reversal potential, $\mathrm{V}_{\text {rev, }}$, of macroscopic tail currents was analyzed in response to hyperpolarizations from -140 to $+40 \mathrm{mV}$ after a brief depolarization to $40 \mathrm{mV}$ (to achieve maximal channel opening), in the presence of different extracellular potassium concentrations $\left(\left[\mathrm{K}^{+}\right]_{\mathrm{o}}\right)$.

A.

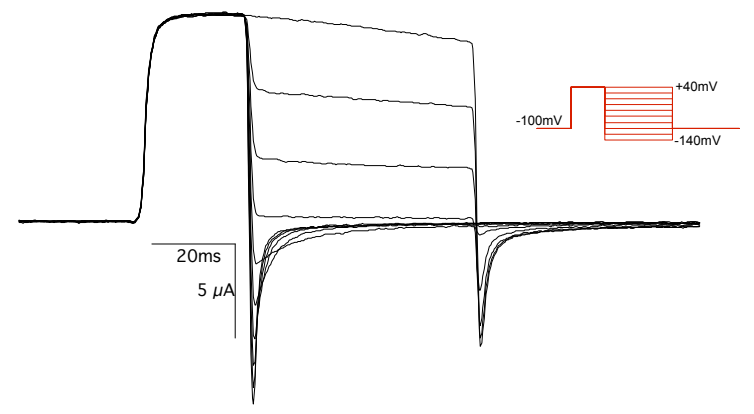

B.

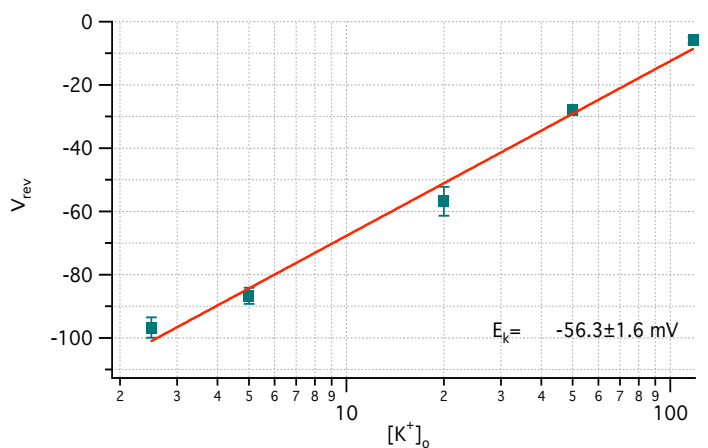

Figure 22. Potassium concentration dependence of the reversal potential of outward mKv1.7 currents. A. Macroscopic tail currents recorded from an oocyte injected with Kcna7 cRNA and bathed with $50 \mathrm{mM} \mathrm{K}^{+}$-Ringer. Following a depolarizing pulse to $+40 \mathrm{mV}$, pulses were applied from -140 to $+40 \mathrm{mV}$ in $20 \mathrm{mV}$ steps at $15 \mathrm{sec}$ inter-pulse intervals. B. Reversal potentials obtained from tail current analysis. Current reversal potentials plotted as a function of $\left[K^{+}\right]_{0}(n=$ 6). The $\mathrm{K}^{+}$concentration was raised replacing $\mathrm{Na}^{+}$with isoosmolar amounts of $\mathrm{K}^{+}$.

$\left[\mathrm{K}^{+}\right]$o were attained by substituting $115 \mathrm{mM} \mathrm{NaCl}$ for the equivalent amount of potassium chloride $(\mathrm{KCl})$ in the bath solution keeping the sum between $\left[\mathrm{K}^{+}\right]$and $\left[\mathrm{Na}^{+}\right]$ at $117.5 \mathrm{mM}$. Figure $22 \mathrm{~A}$ shows representative tail currents recorded from oocytes expressing mKv1.7 wt channels. Macroscopic tail currents from wild type and mKv1.7-T0 channels were indistinguishable and decayed after a depolarizing pulse to $+40 \mathrm{mV}$ that ensured maximum channel opening (tail pulse protocol given in the inset). The external potassium concentration $\left[\mathrm{K}^{+}\right]_{0}$ was $50 \mathrm{mM}\left(67.5 \mathrm{mM} \mathrm{Na}^{+}\right)$, and tail currents from this experiment reversed direction at about $-30 \mathrm{mV}$. The value of the reversal potential changed following the external potassium concentration and was the same between wild type and mutant channels as predicted from identical pore sequences. The dependence of the $\mathrm{V}_{\text {rev }}$ on $\left[\mathrm{K}^{+}\right]_{0}$ was plotted on semi-logarithmic scale for $\left[\mathrm{K}^{+}\right]_{\circ}$ of $2.5,5,20,50$ and $117.5 \mathrm{mM} \mathrm{K}^{+}$. The data were well fitted by the 
Nernst equation function giving a slope of $-55.11 \mathrm{mV}$ in the experiment given in $A$, and an average measured reversal potential of $-56.3 \pm 1.6 \mathrm{mV}$ for a 10 -fold change in $\left[\mathrm{K}^{+}\right]_{\mathrm{o}}$ for 6 different oocytes (Fig.22B). Both values are in good agreement with the Nernst equilibrium potential for a perfectly $\mathrm{K}^{+}$selective pore at room temperature (18$21^{\circ} \mathrm{C}$ ). Thus indicating that the mKv1.7 channels have a higher selectivity for $\mathrm{K}^{+}$over $\mathrm{Na}^{+}$and $\mathrm{Cl}^{-}$, the other nominal monovalent ions present in the bath solution.

\subsubsection{Influence of the extracellular ions}

During TEVC experiments it is virtually impossible to achieve zero $\left[\mathrm{K}^{+}\right]_{\mathrm{o}}$ due to leak of $\mathrm{K}^{+}$from the oocyte and accumulation of the ions in the microvilli on the oocyte surface therefore we refer to the minimal $\left[\mathrm{K}^{+}\right]_{0}$ where no exogenous $\mathrm{K}^{+}$is present in the bath solution as nominal $0 \mathrm{mM} \mathrm{K}^{+}$. When $117.5 \mathrm{mM} \mathrm{Cs}^{+}, \mathrm{Li}^{+}, \mathrm{Na}^{+}, \mathrm{NH}_{4}{ }^{+}$or $\mathrm{Tris}^{+}$ was applied to the extracellular medium (nominal $\left[\mathrm{K}^{+}\right]_{0}=0 \mathrm{mM}$ ) no evident effect on the amplitude or kinetics of the evoked currents mediated by $\mathrm{mKv} 1.7$ channels, both wild type and T0 was observed (data not shown). Accordingly, the addition of increasing concentrations of $\mathrm{K}^{+}$or $\mathrm{Rb}^{+}$to the bath generated an evident decrease in the current that could be rescued by perfusing the recording chamber with $117.5 \mathrm{mM}$ $\mathrm{Na}^{+}$or NFR. Figure 23A and $\mathrm{B}$ show representative mKv1.7 wt and mKv1.7-T0 currents of single experiments where the bath solution was exchanged consecutively with $2.5,5,20,50$ and $117.5 \mathrm{mM} \mathrm{K}^{+}$(all solutions had $\mathrm{Na}^{+}$in order to keep $\left.\left[\mathrm{K}^{+}\right]+\left[\mathrm{Na}^{+}\right]=117.5 \mathrm{mM}\right)$. From the current traces it is evident that high concentrations of potassium ions exerted a marked effect on mKv1.7 currents that only partially can be explained by the reduction on the driving force for $\mathrm{K}^{+}$ions. The dose dependent modulation of peak current amplitude of Kv1.7 channels is given in figure 23C.

Analysis of the fractional current at $+40 \mathrm{mV}$, determined by the ratio between the peak current in different external potassium concentrations and the peak current under control conditions $\left(\mathrm{I}_{\mathrm{K}^{+}} / \mathrm{I}_{\mathrm{NFR}}\right)$ plotted against the $\left[\mathrm{K}^{+}\right]_{\mathrm{o}}$, made evident that most likely there is some particular effect of $\mathrm{K}^{+}$on the cloned $\mathrm{Kv} 1.7$ channels since at intermediate concentrations it deviates from the theoretical expected behavior (Fig.23C). Furthermore, when the relative open probabilities for each potassium 
A.

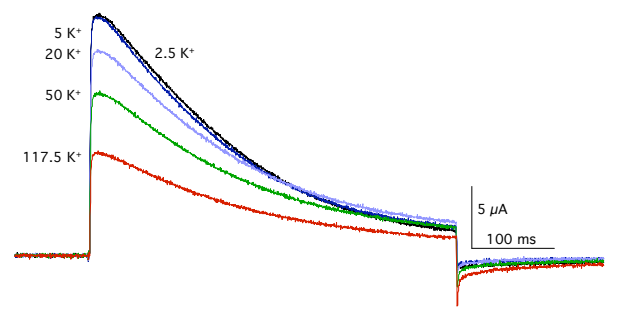

C.

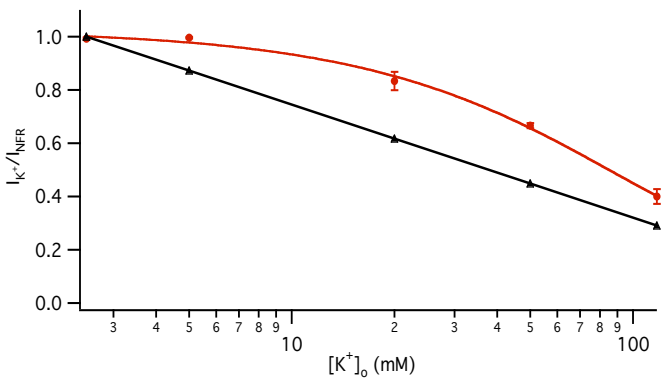

E.

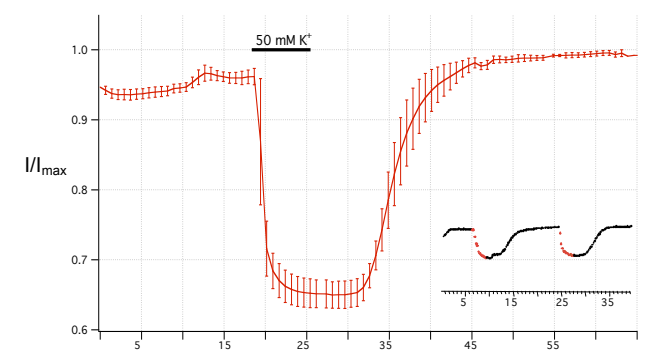

B.
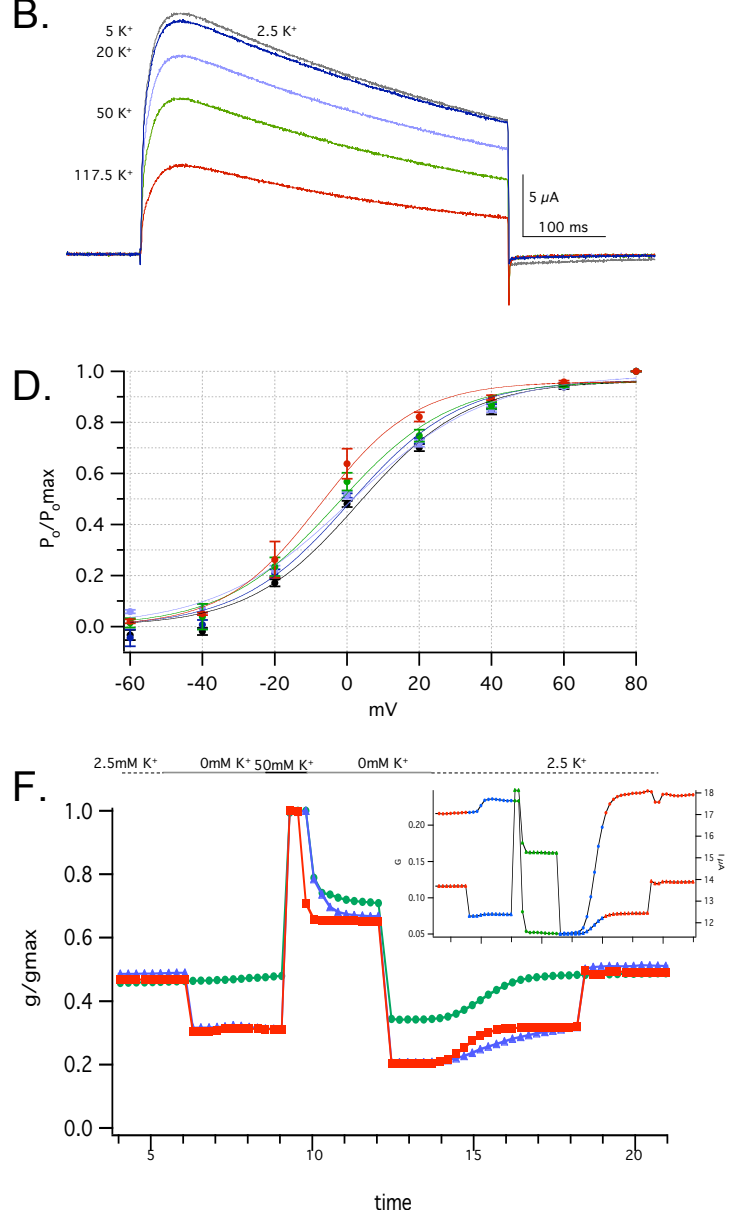

Figure 23. Representative current traces at $+40 \mathrm{mV}$ from $\mathrm{mKv} 1.7 \mathrm{wt}$ and $\mathrm{mKv} 1.7-\mathrm{TO}$ exposed to extracellular increasing potassium concentrations of $2.5 \mathrm{mM}\left(115 \mathrm{mM} \mathrm{Na}^{+}, \mathrm{NFR}\right.$, black), $5 \mathrm{mM}\left(112.5 \mathrm{Na}^{+}\right.$, dark blue), $20 \mathrm{mM}\left(97.5 \mathrm{Na}^{+}\right.$, light blue), $50 \mathrm{mM}\left(67.5 \mathrm{mM} \mathrm{Na}^{+}\right.$, green), and $117.5 \mathrm{mM}\left(0 \mathrm{mM} \mathrm{Na}^{+}\right.$, red). A. mKv1.7 wt. B. mKv1.7-T0. C. Current decrease as a function of the potassium concentration. Peak currents under different potassium concentrations, $\mathrm{I}_{\mathrm{K}}^{+}$, were divided by peak currents under control conditions, $\mathrm{I}_{\mathrm{NFR}}$, and plotted against the $\left[\mathrm{K}^{+}\right]_{0}$ (red squares, $\mathrm{n}=5$ ). The theoretical $\mathrm{I}_{\mathrm{NFR}} / \mathrm{I}_{\mathrm{K}}^{+}$was calculated as stated in methods (black triangles). D. Steady state activation curves from each $\mathrm{K}^{+}$concentration tested, colors as in $A$ and $B$. The plot shows mean values from 6 cells where $\left[\mathrm{K}^{+}\right]_{\mathrm{o}}$ was increased sequentially during each experiment. E. Time course of the application of a $50 \mathrm{mM}$ $\mathrm{K}^{+}\left(67.5 \mathrm{mM} \mathrm{Na}^{+}\right)$to the bath. Values are mean \pm sem from 4 oocytes. Continuous perfusion of NFR was briefly changed to $50 \mathrm{mM} \mathrm{K+}$ in the time indicated with the bar. F. Time course of the conductance during the exchange of solutions. Three experiments are shown where $\left[\mathrm{K}^{+}\right]_{\mathrm{o}}$ was changed. In green circles (cell 1 ) is represented one experiment where perfusion of $2.5 \mathrm{~K}^{+}$with a short pulse of $50 \mathrm{mM} \mathrm{K}$ and wash with $2.5^{+} \mathrm{K}$ was tested. Red squares (cell 2) and blue triangles (cell 3 ) conductance changes of two different cells were exposed to $2.5 \mathrm{mM} \mathrm{K}^{+}, 0 \mathrm{mM} \mathrm{K}^{+}, 50 \mathrm{mM} \mathrm{K}^{+}, 0 \mathrm{mM} \mathrm{K}^{+}$and $2.5 \mathrm{mM} \mathrm{K}^{+}$. In the inset an overlay of the conductance (left axis) and the current (right axis) for cell 3 is shown.

concentration used was determined, no significant change in the $V_{1 / 2}$ or voltage dependence in the concentration range of potassium tested could be observed (Fig.23D). Figure 23E shows the time course of the "wash in" of a solution containing $50 \mathrm{mM} \mathrm{K}^{+}$. To achieve this we implemented a protocol where a train of $500 \mathrm{~ms}$ pulses 
to $+40 \mathrm{mV}$, from a $\mathrm{V}_{\mathrm{h}}$ of $-100 \mathrm{mV}$ every 15 seconds (frequency of $0.25 \mathrm{~Hz}$ ) was applied while perfusing continuously the different solutions. The perfusion speed was kept constant over the whole experiment. The resulting trace in Fig.23E is the average of experiments from 4 different oocytes expressing mKv1.7 channels. The black bar indicates the time where $50 \mathrm{mM} \mathrm{K}^{+}$solution was perfused, in the inset are given two identical pulses of $50 \mathrm{mM} \mathrm{K}^{+}$and its correspondent washes (in red and black, respectively) showing that the effect was reproducible and washable over the same time lapse. Plots of the conductance calculated for this train of pulses from three different cells are shown in the panel $\mathrm{F}$ of figure 23 . In green circles the experiment from $E$ is illustrated, whereas the blue triangles and red squares represent two other cells that were exposed to zero $\mathrm{K}^{+}$before and after the $50 \mathrm{mM} \mathrm{K}^{+}$pulse (consecutively: $2.5,0,50,0$, and $2.5 \mathrm{mM}$ potassium as shown). In the inset of this panel an overlay of the time course of the current and conductance changes is given for easier comparison. Interestingly $\mathrm{K}^{+}$and $\mathrm{Rb}^{+}$concentrations higher than $50 \mathrm{mM}$ led to an irreversible reduction of the currents.

\subsubsection{Activation of mKv1.7 channels}

$\mathrm{Kv}$ channels undergo conformational rearrangements that lead to channel opening evidenced by the flow of ionic currents, a process recognized as activation. The primary response to graded depolarization of mKv1.7 channels was indeed opening or activation that conceptually could be related to two aspects, voltage sensing and gating, that in voltage gated ion channels are known to be energetically coupled in a way that changes in the voltage alter the open probability $\left(P_{o}\right)$ of the channels. Activation kinetics were evaluated by determining the time required to reach $75 \%$ of the peak current $\left(\mathrm{RT}_{75}\right)$. Figure $24 \mathrm{~A}$ and $\mathrm{C}$ present the initial times $(\sim 50 \mathrm{~ms})$ of envelopes of current traces elicited by the IV protocol explained before. In A superimposed traces of TEVC recordings of mKv1.7 wt and mKv1.7-T0 channels are displayed while in $\mathrm{C}$ on cell patch clamp recordings from oocytes expressing the same channels are shown. The quantification of the RT $T_{75}$ from TEVC experiments is comprised within the plots of the voltage dependence of the $\mathrm{RT}_{75}$ of mKv1.7 wt channels and mKv1.7-T0 versus the command potential in panel B and table 3 . 
A

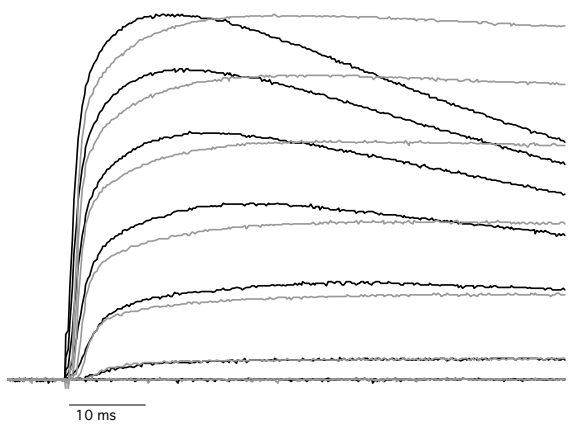

C.

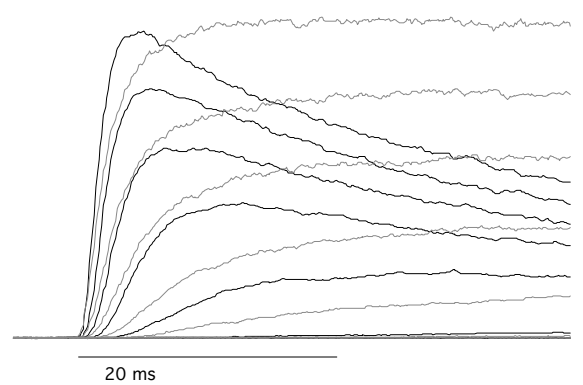

B.

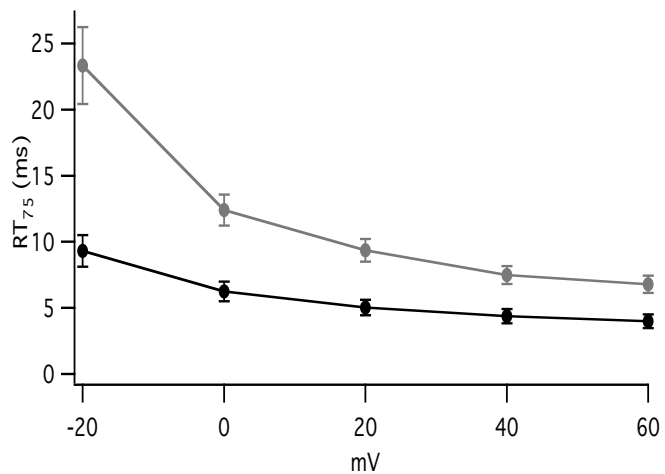

D.

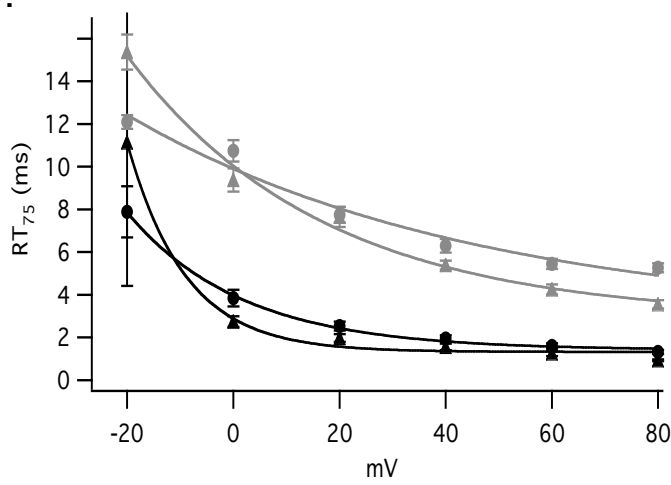

Figure 24. Activation kinetics of mKv1.7 wt and mKv1.7-T0. A. The first 50ms of normalized and superimposed current traces measured with TEVC from oocytes expressing mKv1.7 wt and mKv1.7-T0 channels are shown in black and gray, respectively. C. On cell patch clamp records of mKv1.7 wt and mKv1.7-T0, first 40ms are shown with colors as in A. Test pulses between -60 and +80 in $20 \mathrm{mV}$ steps for TEVC and patch experiments. B. Rise time plot of mKv1.7 wt $(n=13)$ and mKv1.7-T0 $(n=20)$ currents measured as the time in ms required to reach $75 \%$ of $I_{\max }, \mathrm{RT}_{75}$ from TEVC experiments. D. Rise time plot (determined as in B.) of mKv1.7 wt and mKv1.7-T0 currents from patch clamp experiments. Circles represent $\mathrm{RT}_{75}$ for on cell measurement and triangles inside out experiments (mKv1.7 wt on cell $\mathrm{n}=8$, inside out $\mathrm{n}=6 ; \mathrm{mKv} 1.7-\mathrm{T0}$ on cell $\mathrm{n}=5$, inside out $\mathrm{n}=4$ ). Colors as in $\mathrm{A}$.

From patch clamp experiments another feature was investigated comparing the mean values obtained for measurements performed in the on cell and inside out configurations (attached and excised patches, respectively). The plot of the patch data is summarized in the panel $\mathrm{D}$ (Fig.24) and table 3. The mean value of $\mathrm{RT}_{75}$ for the $\mathrm{mKv} 1.7$ wt was $10 \mathrm{~ms}$ at $-20 \mathrm{mV}$ and $4 \mathrm{~ms}$ at $+60 \mathrm{mV}$ while for mKv1.7-T0 currents reached $75 \%$ of the peak within $23 \mathrm{~ms}$ during TEVC experiments. As it can be seen the rise times to $75 \%$ of the peak current are significantly different between wild type and $\mathrm{N}$-terminus mutants having the last ones $\mathrm{RT}_{75}$ more than 1.5 times the values observed for mKv1.7 wt within the range of potentials evaluated (-20 to $+60 \mathrm{mV})$.

From patch clamp experiments performed to evaluate the $\mathrm{RT}_{75}$ the same significant difference between mKv1.7 wt and mKv1.7-T0 currents was observed. 
Moreover, no significant differences could be detected in $\mathrm{RT}_{75}$ from experiments in the on cell and inside out configurations of the patch clamp technique. Nevertheless, rise times observed from patch clamp experiments were slightly smaller for all the potentials tested than the equivalent data obtained from TEVC. Two-tailed $p$ value for the comparison between the TEVC data and the corresponding patch data sets for each channel is bigger than 0.05 indicating that this is not statistically different.

Table 3.

Rise times to $75 \%$ of the peak currents determined by TEVC measurements

\begin{tabular}{cccc}
\hline \multicolumn{4}{c}{$\mathrm{RT}_{75}(\mathrm{~ms})$} \\
\hline & mKv1.7 wt & mKv1.7-T0 & $\mathrm{p}^{*}$ \\
$\mathrm{~V}_{\mathrm{m}}$ & Mean \pm sem & mean \pm sem & \\
\hline$-20 \mathrm{mV}$ & $9.31 \pm 1.2$ & $23.3 \pm 2.9$ & 0.0002 \\
$0 \mathrm{mV}$ & $6.25 \pm 0.8$ & $12.4 \pm 1.2$ & 0.0002 \\
$20 \mathrm{mV}$ & $5.02 \pm 0.6$ & $9.36 \pm 0.9$ & 0.0004 \\
$40 \mathrm{mV}$ & $4.37 \pm 0.5$ & $7.47 \pm 0.7$ & 0.0015 \\
$60 \mathrm{mV}$ & $4.00 \pm 0.5$ & $6.78 \pm 0.7$ & 0.0072 \\
\hline $\mathrm{n}$ & 12 & 12 & \\
\hline p corresponds to the comparison between Kv1.7 wt and Kv1.7- \\
T0 channels.
\end{tabular}

Table 4.

Rise times to $75 \%$ of the peak currents determined

by Patch clamp measurements

\begin{tabular}{|c|c|c|c|c|c|}
\hline & & $\mathrm{RT}_{75}(\mathrm{~ms})$ & & & \\
\hline & $\begin{array}{c}\mathrm{mKv} 1.7 \text { wt } \\
\text { On cell }\end{array}$ & $\begin{array}{l}\mathrm{mKv} 1.7 \mathrm{wt} \\
\text { Inside out }\end{array}$ & $\begin{array}{c}\text { mKv1.7-T0 } \\
\text { On cell }\end{array}$ & $\begin{array}{l}\text { mKv1.7-T0 } \\
\text { Inside out }\end{array}$ & $\mathrm{p}$ \\
\hline $\mathrm{V}_{\mathrm{m}}$ & mean \pm sem & Mean \pm sem & mean \pm sem & Mean \pm sem & \\
\hline$-20 m V$ & $7.88 \pm 1.2$ & $11.10 \pm 6.7^{\mathrm{ns}}$ & $12.10 \pm 0.3$ & $15.40 \pm 0.8^{\mathrm{ns}}$ & 0.0051 \\
\hline $0 \mathrm{mV}$ & $3.85 \pm 0.4$ & $2.75 \pm 0.3^{\text {ns }}$ & $10.70 \pm 0.5$ & $9.37 \pm 0.5^{\mathrm{ns}}$ & 0.0001 \\
\hline $20 \mathrm{mV}$ & $2.53 \pm 0.2$ & $1.97 \pm 0.2^{\mathrm{ns}}$ & $7.75 \pm 0.4$ & $7.66 \pm 0.5^{\mathrm{ns}}$ & 0.0001 \\
\hline $40 \mathrm{mV}$ & $1.95 \pm 0.2$ & $1.56 \pm 0.2^{\mathrm{ns}}$ & $6.30 \pm 0.3$ & $5.40 \pm 0.2^{\mathrm{ns}}$ & 0.0001 \\
\hline $60 \mathrm{mV}$ & $1.61 \pm 0.1$ & $1.26 \pm 0.1^{\mathrm{ns}}$ & $5.45 \pm 0.2$ & $4.25 \pm 0.2^{\mathrm{ns}}$ & 0.0001 \\
\hline $\mathrm{n}$ & 15 & 9 & 12 & 4 & \\
\hline
\end{tabular}




\subsubsection{Inactivation of $\mathrm{mKv} 1.7$ channels}

After activation and in response to an extended depolarization, ion channels can enter in a state where no ionic flow is further possible before the channels enter a recovery period that is highly dependent on the time and the potential. This process is recognized as inactivation and it can occur in very different time frames being extremely fast in the order of milliseconds (N-type) or very slow (C-type) in the seconds range (and up to tens of seconds). The inactivation development is regarded as a characteristic feature particular of each Kv channel class. The protein sequences of wild type and T0 channels are virtually identical with the exception that mKv1.7-T0 channels lack the first 32 amino acids in its $\mathrm{N}$-terminus. The $\mathrm{N}$-terminal domain of mKv1.7 channels is assumed to reside in the cytoplasmic side of the membrane from protein domain analysis. Such feature suggested the possibility of observing a "N-type inactivation like" process for mKv1.t wt that could be disrupted or altered in the $\mathrm{N}$-terminus mutant mKv1.7-T0. Differences in the current kinetics, fundamentally in the inactivation process, between mKv1.7 wt and mKv1.7-T0 channels were expected. That indeed was the case when we corroborated that the main dissimilarity between mKv1.7 wt and mKv1.7-T0 channels was a marked difference in the time course of the inactivation process. Figure 25 offers a good example of such dissemblances by overlaying current traces in response to long depolarization of the mouse wild type and N-terminus mutant channels. In Fig.25A current traces in response to an IV protocol of 2.5 seconds of both channel types are scaled and superimposed. The mKv1.7 wt channels inactivated fast and completely (less than $5 \%$ steady state current left) at the end of a $2.5 \mathrm{sec}$ depolarization to $+40 \mathrm{mV}$, whereas mKv1.7-T0 channels after $2.5 \mathrm{sec}$ exhibited $27 \%$ of current that was resistant to inactivation (Fig.25, inset panel $A$ ).

The quantification of the inactivation process under control conditions (normal frog ringer, NFR, $2.5 \mathrm{mM} \mathrm{K}^{+} / 115 \mathrm{mM} \mathrm{Na}^{+}$) was assessed by fitting to a monoexponential function the decay of outward currents observed in response to prolonged IV stimulation protocol. The current decay that was used for the fit comprised between 50 and 500ms for mKv1.7 wt and 50 and 2500ms for mKv1.7-T0. For both channel types a function of a single exponential was sufficient to describe the inactivation process. 
A.

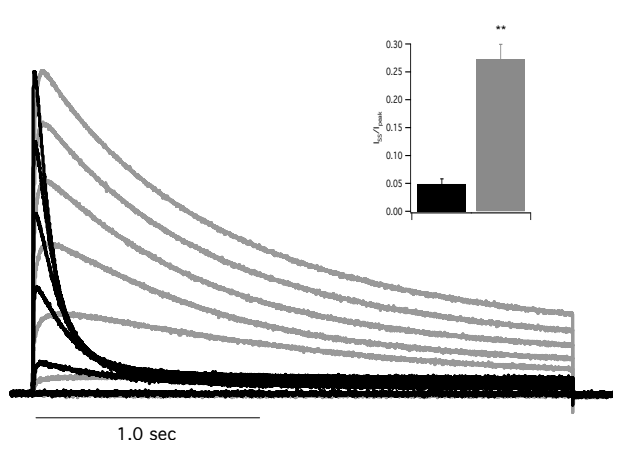

B.

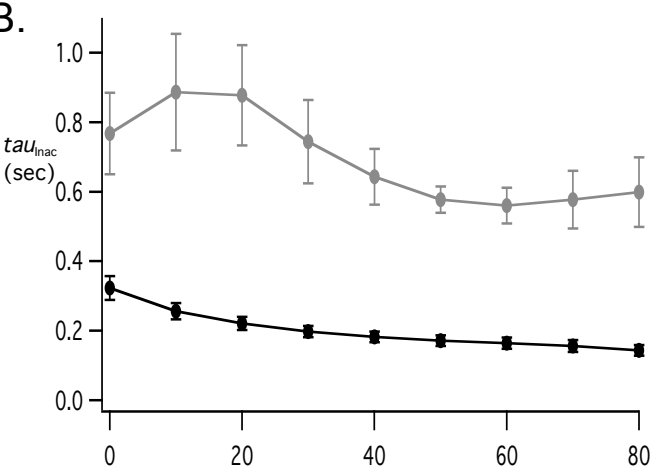

$\mathrm{mV}$

Figure 25. Inactivation kinetics of mKv1.7 wt and mKv1.7-T0. A. Normalized and superimposed mKv1.7 wt and mKv1.7-T0 current traces elicited by a $2.5 \mathrm{sec}$ pulse to pulses between -60 and $+80 \mathrm{mV}$ in $20 \mathrm{mV}$ steps are shown in black for mKv1.7 wt and gray for mKv1.7-T0. The bar diagram in the inset summarizes the plot of the fraction steady state current amplitude and peak current amplitude $I_{s s} / I_{\text {peak }}$ of wild type (black) and T0 mutants (gray). B. Inactivation time constants derived from mono-exponential function fits to the inactivation processes; mKv1.7 wt $(n=15)$ and mKv1.7-T0 $(n=13)$. Inset shows the part of the current decay that was fitted, colors as in $A$.

The time constants derived from the analysis, $t a u_{\text {Inac }}$, were plotted versus the membrane potential as represented in Fig.25B. Statistical analysis of the $\operatorname{tau}_{\text {Inac }}$ of Kv1.7 wt currents over all the potentials evaluated ( 0 to $80 \mathrm{mV}$ ) showed to be significantly faster than the time constants obtained from currents elicited by mKv1.7T0 channels ( $\operatorname{tau}_{\text {Inac }}$ at $+40 \mathrm{mV}$ of $182 \pm 16$ and $643 \pm 80 \mathrm{~ms}$, respectively). Moreover, time constants of inactivation were monotonic and poorly voltage dependent, being slightly larger at threshold potentials. Due to the shallow voltage dependence and the rather large variability, shifts in the voltage dependence of the time constants could not be determined.

Table 5.

Inactivation time constants $\operatorname{ta}_{\mathrm{Inac}}$ of $\mathrm{mKv} 1.7 \mathrm{wt}$ and $\mathrm{mKv} 1.7-\mathrm{T} 0$ determined by TEVC

\begin{tabular}{cccc}
\hline \multicolumn{3}{c}{$\operatorname{tau}_{\text {Inac }}(\mathrm{ms})$} \\
\hline & $\begin{array}{c}\text { mKv1.7 wt } \\
\text { mKv1.7-T0 }\end{array}$ & $\mathrm{P}$ \\
$\mathrm{V}_{\mathrm{m}}$ & Mean \pm sem & mean \pm sem & \\
\hline $0 \mathrm{mV}$ & $322.8 \pm 34.6$ & $767.5 \pm 116.9$ & 0.0072 \\
$20 \mathrm{mV}$ & $221.1 \pm 18.7$ & $877.4 \pm 144.0$ & 0.0015 \\
$40 \mathrm{mV}$ & $181.8 \pm 15.4$ & $643.2 \pm 80.1$ & 0.0001 \\
$60 \mathrm{mV}$ & $164.5 \pm 15.8$ & $560.1 \pm 51.5$ & 0.0001 \\
\hline $\mathrm{n}$ & 15 & 13 & \\
\hline
\end{tabular}


A.

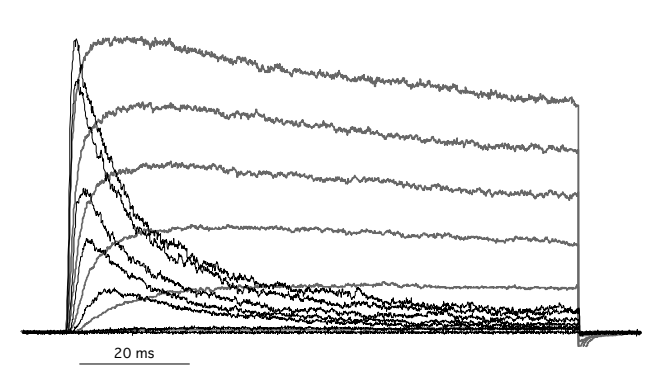

C.

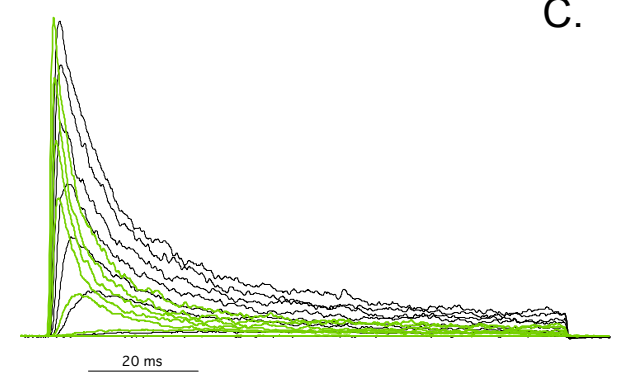

B.

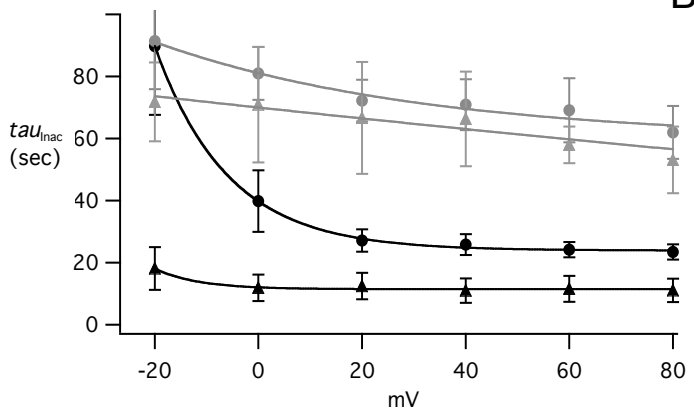

D.

Figure 26. Inactivation kinetics from patch clamp experiments of mKv1.7 wt and mKv1.7T0. A. On cell patch clamp current traces from oocytes expressing mKv1.7 wt and mKv1.7-T0 channels are shown in black and gray, respectively. Test pulses between -60 and $+80 \mathrm{mV}$ in $20 \mathrm{mV}$ steps; $\mathrm{V}_{\mathrm{h}}=-100 \mathrm{mV}$. B. Inactivation time constants derived from mono-exponential fit functions to the inactivation processes, $\operatorname{tau}_{\text {inac }}$, plot of mKv1.7 wt in black (circles represent on cell, $\mathrm{n}=8$, and triangles inside out, $\mathrm{n}=6$, patches); and mKv1.7T0 currents in gray (circles denote on cell $n=5$, and triangles inside out, $n=4$, current records). C and D. Superimposed on cell and inside out patch clamp records of the same patch containing mKv1.7 wt and mKv1.7-T0 channels (black and gray are on cell, green is inside out).

The currents measured during patch clamp experiments seem to be faster than the TEVC equivalents (compare Fig.25 and Fig.26A and B.), a feature previously noticed during this work (see $\mathrm{RT}_{75}$ determination). This is often observed and explained by the fact that with the patch technique the quality and speed of the clamp is highly improved. Although faster, $\operatorname{tau}_{\text {Inac }}$ were again in good correspondence with the TEVC data. This is further evidence that wild type channels mediated currents are in fact about 2.5 times faster than the T0 mutants (mKv1.7 wt tau Inac $=$ $26 \pm 4 \mathrm{~ms}$ vs mKv1.7-T0 $\operatorname{tau}_{\mathrm{Inac}}=66 \pm 3 \mathrm{~ms}$ respectively for experiments in the on cell configuration at $+40 \mathrm{mV}$, Fig. $26 \mathrm{~A}$ and B.). The $\operatorname{tau}_{\mathrm{Inac}}$ data obtained with the patch clamp technique is contained in Fig. $26 \mathrm{C}$ and $\mathrm{D}$, as well as in Table 6. There it can be observed that when the patches were excised, that is, changed from the on cell to the inside out excised patch configuration, mKv1.7 wt channels inactivated faster than mKv1.7-T0. For instance, from pulses to $+40 \mathrm{mV}$ wild type currents from inside out patches decayed with an average time constant of $11 \pm 4 \mathrm{~ms}$, less than half the on 
cell tau $u_{\text {Inac }}$ from the same potential (significance, $p=0.03$ ). Although currents from mKv1.7-T0 inside out patches appeared a bit faster than the currents from on cell measurements, no significant difference could be found through the statistic analysis $\left(\right.$ tau $_{\text {Inac }}$ on cell $71 \pm 8 \mathrm{~ms}$, tau $u_{\text {Inac }}$ inside out $\left.66 \pm 3 \mathrm{~ms}, \mathrm{p}=0.731\right)$. On this basis, we suggest that the presence of the 32 amino acids in the $\mathrm{N}$-terminus of mKv1.7 wt has an enhancing effect on the onset of the inactivation.

Interestingly, oocytes expressing mKv1.7-T0 channels were somehow more difficult to patch than mKv1.7 wt expressing oocytes. Furthermore, the patches from the mutant channels were often unstable making it harder to perform measurements of the same patch in both configurations (not investigated further).

Table 6.

Inactivation time constants $\operatorname{tau}_{\mathrm{Inac}}$ of $\mathrm{mKv} 1.7 \mathrm{wt}$ and mKv1.7-T0 determined by patch clamp

\begin{tabular}{|c|c|c|c|c|c|}
\hline & & $\operatorname{tau}_{\text {Inac }}(\mathrm{ms})$ & & & \\
\hline & $\begin{array}{c}\mathrm{mKv} 1.7 \mathrm{wt} \\
\text { On cell }\end{array}$ & $\begin{array}{c}\mathrm{mKv} 1.7 \mathrm{wt} \\
\text { Inside out }\end{array}$ & $\begin{array}{c}\mathrm{mKv} 1.7-\mathrm{T0} \\
\text { On cell }\end{array}$ & $\begin{array}{c}\mathrm{mKv} 1.7-\mathrm{T0} \\
\text { Inside out }\end{array}$ & $p$ \\
\hline $\mathrm{V}_{\mathrm{m}}$ & Mean \pm sem & Mean \pm sem & Mean \pm sem & Mean \pm sem & \\
\hline $0 \mathrm{mV}$ & $39.9 \pm 9.95$ & $11.9 \pm 4.3^{*}$ & $81.0 \pm 8.5$ & $70.9 \pm 18.6^{\text {ns }}$ & 0.0054 \\
\hline $20 \mathrm{mV}$ & $27.1 \pm 3.6$ & $12.5 \pm 4.3^{*}$ & $72.2 \pm 6.7$ & $66.6 \pm 18.1^{\text {ns }}$ & 0.0001 \\
\hline $40 \mathrm{mV}$ & $25.8 \pm 3.4$ & $11.0 \pm 3.9^{*}$ & $70.9 \pm 8.2$ & $66.3 \pm 15.3^{\text {ns }}$ & 0.0001 \\
\hline $60 \mathrm{mV}$ & $24.2 \pm 2.5$ & $11.6 \pm 4.2^{*}$ & $69.1 \pm 10.3$ & $58.0 \pm 5.6^{\text {ns }}$ & 0.0001 \\
\hline $\mathrm{N}$ & 15 & 9 & 12 & 4 & \\
\hline
\end{tabular}

\subsection{Effect of extracellular potassium on the inactivation}

Several observations suggest that the inactivation of mKv1.7 channels seems to be an interesting and highly regulated process. Therefore we decided to 
A.
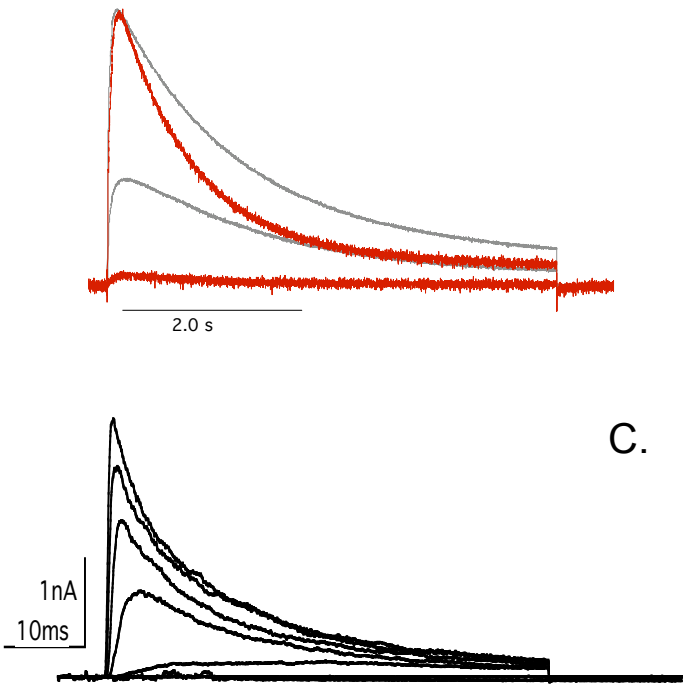

E.

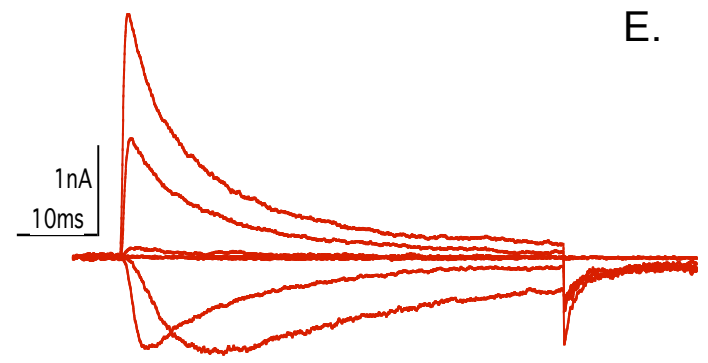

B.
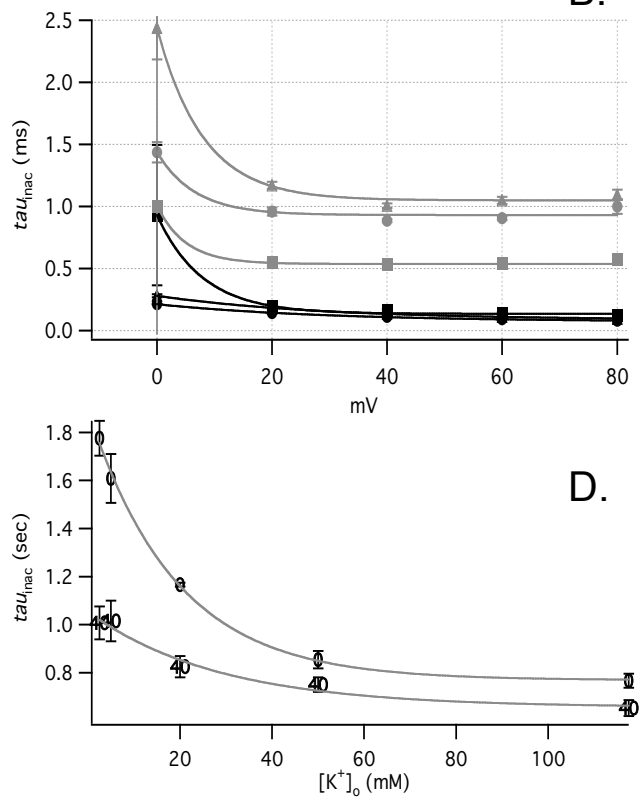

F.

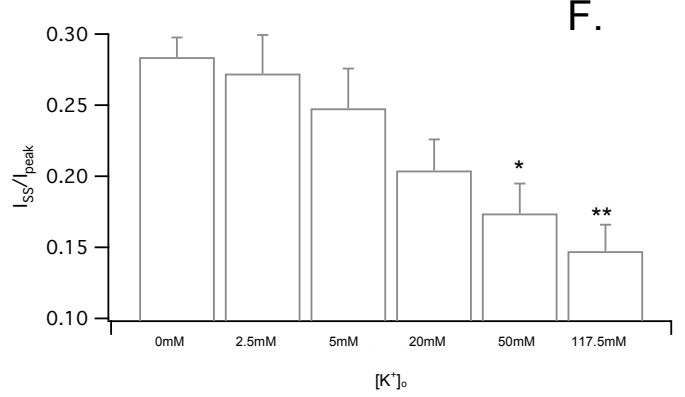

Figure 27. Inactivation kinetics of mKv1.7 wt and mKv1.7-T0. A. Shows normalized mKv1.7TO current traces at 0 and $+40 \mathrm{mV}$ of $2.5 \mathrm{mM} \mathrm{K}^{+}\left(115 \mathrm{mM} \mathrm{Na}^{+}\right)$in gray, and $117.5 \mathrm{mM} \mathrm{K}^{+}(0 \mathrm{mM}$ $\mathrm{Na}^{+}$) in red. B. Inactivation time constants derived from mono-exponential fits to the inactivation processes, $t_{a} u_{\text {inac. }}$ Plot of mKv1.7 wt (black) and mKv1.7-T0 (gray) currents. Recordings under $0 \mathrm{mM} \mathrm{K}^{+}\left(117.5 \mathrm{mM} \mathrm{Na}^{+}\right)$are designated with triangles, $2.5 \mathrm{mM} \mathrm{K}^{+}(115 \mathrm{mM}$ $\mathrm{Na}^{+}$) with circles, and $117.5 \mathrm{mM} \mathrm{K}^{+}\left(0 \mathrm{mM} \mathrm{Na}^{+}\right.$, with squares) ( $n=5$ per channel type and condition). C. and E. On cell patch clamp recordings of two different oocytes expressing mKv1.7 wt channels, in $\mathrm{C}$. the patch pipette was filled with $2.5 \mathrm{mM} \mathrm{K}^{+}$, while in $\mathrm{E}$. the patch pipette contained $117.5 \mathrm{mM} \mathrm{K}^{+}$, the IV protocol consisted of $100 \mathrm{~ms}$ pulses from -60 to $+60 \mathrm{mV}$ in $20 \mathrm{mV}$ steps from a $V_{h}=-100 \mathrm{mV}$. D. Plot of the $\left[\mathrm{K}^{+}\right]_{0}$ dependence of the $\tan _{\text {inac }}$ of $\mathrm{mKv} 1.7-$ T0 currents elicited at 0 and $+40 \mathrm{mV}$. F. Residual current after $4.5 \mathrm{sec}$ depolarizing pulse to $+40 \mathrm{mV}$. Plot of the ratio between steady state current amplitude and peak current amplitude $\mathrm{I}_{\mathrm{ss}} / \mathrm{I}_{\text {peak }}$ for 4 oocytes expressing mKv1.7-T0 channels exposed to different potassium concentrations.

investigate how inactivation in this channel might be regulated and as a consequence the influence of the presence of different concentrations of extracellular potassium was assesed. The panel B in Fig.27 shows the plot of the $\operatorname{ta}_{\text {Inac }}$ of experiments where extracellular $\mathrm{K}^{+}$concentrations of $0\left(0\right.$ nominal $\left.\mathrm{K}^{+} / 117.5 \mathrm{mM} \mathrm{Na}^{+}\right), 2.5$ (NFR with $\left.115 \mathrm{mM} \mathrm{Na}^{+}\right)$, and $117.5 \mathrm{mM}\left(\mathrm{K}^{+}\right.$-Ringer with $\left.0 \mathrm{mM} \mathrm{Na}^{+}\right)$were assayed (triangles, 
circles and squares, respectively). The black symbols and traces correspond to the experiments performed with oocytes expressing mKv1.7 wt channels and the gray traces correspond to mKv1.7-T0. As it can be seen, extreme variations in the $\left[\mathrm{K}^{+}\right]_{\mathrm{o}}$ did not affect inactivation kinetics of wild type Kv1.7 channels. Also from the patch clamp experiments the presence in the pipette of $117.5 \mathrm{mM} \mathrm{K}^{+}$did not alter in a significant manner the $\operatorname{tau}_{\text {Inac }}$ under control conditions of $\mathrm{mKv1.7}$ currents as it can be seen in Figure 27 panel $D$ for an on cell recording of an oocyte expressing mKv1.7 wt channels (patch pipette filled with $2.5 \mathrm{mM} \mathrm{K}^{+}$) and in panel $\mathrm{E}$ for another oocyte patch with a pipette loaded with $117.5 \mathrm{~K}^{+}$(both recordings were made with $117.5 \mathrm{mM} \mathrm{K}^{+}$in the extracellular medium). The mean inactivation time constants for on cell recordings were $\operatorname{ta}_{\text {Inac: }} 26 \pm 4 \mathrm{~ms}$ and $35 \pm 6 \mathrm{~ms}$ for $2.5 \mathrm{mM} \mathrm{K}^{+}$in the pipette (control) and $117.5 \mathrm{mM} \mathrm{K}^{+}$respectively $(\mathrm{p}=0.2170)$. In the presence of $117.5 \mathrm{mM} \mathrm{K}^{+}$the inactivation process of mKv1.7-T0 was about two fold faster, significantly affecting the mutant channel kinetics (panel D, Fig.27). Figure 27B shows the ta $u_{\text {Inac }}$ determined for currents elicited by 0 and $+40 \mathrm{mV}$ pulses against the potassium concentration in which they were determined. The inactivation time constant was decreased by high extracellular potassium at all potentials studied, likewise the effect at $0 \mathrm{mV}$ was particularly more noticeable than at more depolarized potentials (1.9 fold change in $\operatorname{tau}_{\text {Inac }}$ vs 1.4 fold change between 5 and $50 \mathrm{mM} \mathrm{K}^{+}$). Accordingly, the bar diagram in the part $\mathrm{C}$ of Fig.27 shows that the residual current after a depolarization to $+40 \mathrm{mV}$ was significantly decreased from $27 \%$ in NFR $\left(2.5 \mathrm{mM} \mathrm{K}^{+}\right)$to less than $15 \%$ in $\mathrm{K}^{+}$-Ringer $\left(117.5 \mathrm{mM} \mathrm{K}^{+}\right)$.

\subsection{Steady state Inactivation}

The inactivation properties from wild type and mutant channels were investigated by means of double pulse inactivation protocols. An example of one such a protocol is given in figure's $28 \mathrm{~A}$ inset. There, channels were challenged to a brief first activating pulse to $+40 \mathrm{mV}\left(\mathrm{P}_{1}\right)$ after which repolarizations to voltage ranges between -120 and $+20 \mathrm{mV}$ (interpulse interval potential) in $10 \mathrm{mV}$ steps of variable lengths $(200,250,300,500,1000$, and $1500 \mathrm{~ms})$ preceeded a second pulse to $+40 \mathrm{mV}\left(\mathrm{P}_{2}\right)$. The fraction of channels available for activation, thus non-inactivated channels, was obtained from the ratio between $P_{2}$ and $P_{1}\left(P_{2} / P_{1}\right) . P_{2} / P_{1}$ plotted 
A.

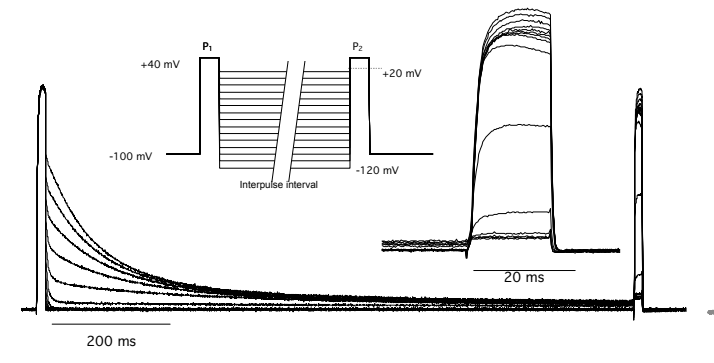

C.

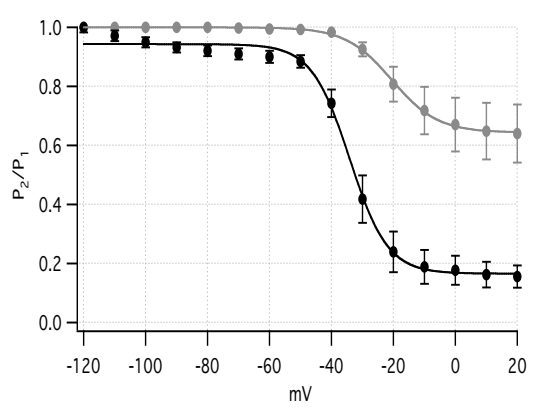

B.

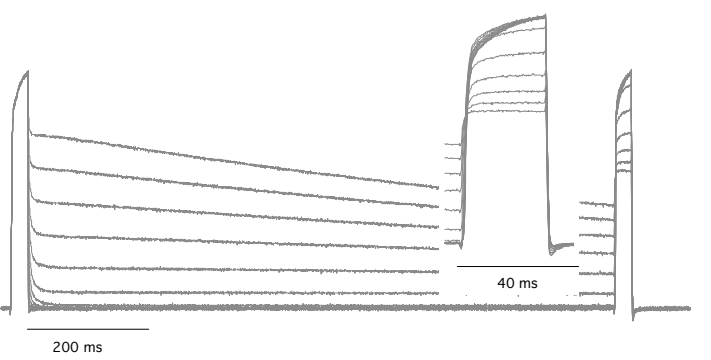

D.

E.

Figure 28. Voltage dependence of the onset of the inactivation of mKv1.7 wt and mKv1.7-T0 channels currents on $X$. laevis oocytes. Inactivation onset was studied by the pulse protocol from the inset: $20 \mathrm{~ms}$ depolarization to $+40 \mathrm{mV}\left(P_{1}\right.$ and $\left.P_{2}\right)$ with interpulse voltages from -120 to $+40 \mathrm{mV}$ in $10 \mathrm{mV}$ for $0.2,0.25,0.3,0.5,1$ and $1.5 \mathrm{sec}$ (interpulse intervals). A and B. Currents recorded using a two-electrode voltage clamp of mKv1.7 wt (black) and mKv1.7-T0 (gray) channels, respectively. $C$. Inactivation estimated as the ratio between pulses to $+40 \mathrm{mV}, \mathrm{P}_{2} / \mathrm{P}_{1}$, indicating the fraction of channels available for activation in $P_{2}$ after the interpulse $(n=6$ and 5 for mKv1.7 wt and mKv1.7-T0, respectively). D. and E. Half inactivation potential and relative rates derived from Boltzmann fits to the $P_{2} / P_{1}$ vs the interpulse interval potential curves, respectively. $P_{2} / P_{1}$ vs potential, $V$, curves were constructed from experiments of different interpulse interval lengths and the resulting of $V_{1 / 2}$ and relative rates plotted against the duration of the interpulse. Black traces represent mKv1.7 wt values and gray corresponds to mKv1.7-T0 (mean \pm sem values are given, $n=6$ for $m K v 1.7$ wt and $n=5$ for mKv1.7-T0).

against the interpulse potential gives rise to curves of the type shown in panel $C$ from Figure 28 that evidence the influence that the prepulse voltage exerts in the onset of the inactivation phenomenon. Prepulse inactivation curves were constructed for both mouse channels for each set of interpulse length data using the two pulses protocol explained before and fitted with a Boltzmann function given by $I_{2} / l_{1}$ or $P_{2} / P_{1}=$ Offset+1/(1+exp $\left.{ }^{((\mathrm{V} 1 / 2-\mathrm{Vm}) / a)}\right)$; where $\mathrm{V}_{1 / 2}$ represents the half inactivation potential and $a$ is the slope parameter that determines the voltage dependence of the process. Figure $28 \mathrm{C}$ includes the mean \pm sem data of experiments from mKv1.7 wt and mKv1.7-T0 mediated currents ( $\mathrm{n}=5$ for each channels, interpulse interval of 1.5 seconds). The average midpoint potential for inactivation of mKv1.7 wt channels was $-40 \pm 0.66 \mathrm{mV}$ with a slope parameter of $4 \pm 0.3$, where after $1.5 \mathrm{sec}$ of interpulse interval $\sim 15 \%$ of the current was resistant to inactivation. Inactivation of T0 was less complete, less voltage dependent and presented a more depolarized $V_{1 / 2}$ for the 
onset of inactivation. Hence, it presented $\sim 65 \%$ non-inactivating component after $1.5 \mathrm{sec}$ interpulse interval; slope equal to $7 \pm 0.7(p=0.0033)$ and midpoint potential of inactivation of $-21 \pm 2 \mathrm{mV}$. Half inactivation potential and slope relative rates derived from Boltzmann fits to the $P_{2} / P_{1}$ vs voltage, were plotted as a function of the interpulse interval time (Figure $28 \mathrm{D}$ and $\mathrm{C}$ ). The resulting $\mathrm{V}_{1 / 2}$ and relative rates turned out to be significantly different for mouse wild type channels at interpulse intervals over $300 \mathrm{~ms}$ (Fig.28 panels $\mathrm{C}$ and D). Along with this results, no particular time dependence of these constants was underlined for Kv1.7-T0 mutant channels but an expectable decrease in the voltage dependence given by the relative rate at interpulse intervals $\geq 1.5 \mathrm{sec}$ (Fig.28 panel E). Both observations are in congruence

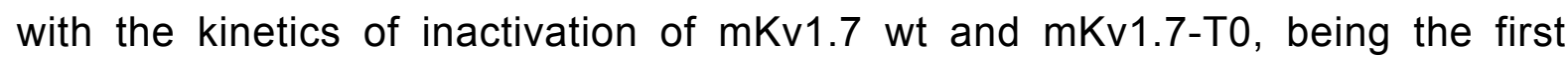
significantly faster than the second. This data indicates that an important effect of the deletion of the N-terminal fragment on the mutant mKv1.7-T0 was to shift to more positive potentials the range of potentials in which inactivation occurred.

\subsection{Effect of extracellular potassium on the steady state inactivation}

For several $\mathrm{K}^{+}$channels it is known that extracellular $\mathrm{K}^{+}$leads to different inactivation kinetics. To study the effects of $\mathrm{K}^{+}$on $\mathrm{mKv} 1.7$ channels prepulse inactivation curves were constructed under control conditions and in the presence of high potassium concentrations $\left(\left[\mathrm{K}^{+}\right]_{0}=50 \mathrm{mM}\right)$ with interpulse intervals of 250,500 and 1500 ms for the murine wild type and mutant channels. From the analysis of half inactivation potentials and voltage dependence changes due to the presence of high $\mathrm{K}^{+}$we could observe that the inactivation process of mKv1.7 channels resulted unaffected by the external potassium concentration (data not shown).

\subsubsection{Cumulative Inactivation of mKv1.7 channels}

During a train of repetitive depolarizing test pulses it has been shown that some $\mathrm{K}^{+}$channels evidence a progressive decline in the amplitude of test pulse currents, a phenomenon called cumulative inactivation. Accordingly, the time course of cumulative inactivation of wild type and mutant mKv1.7-T0 channels was 

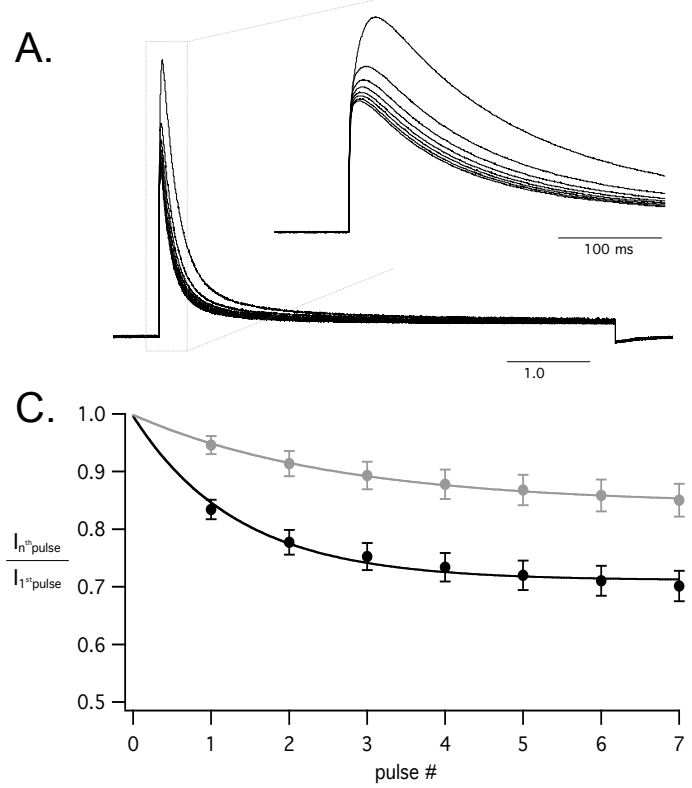

B.
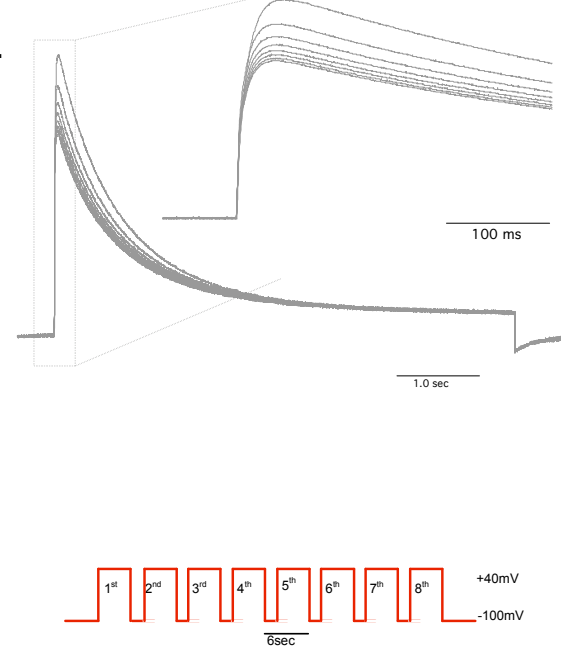

Figure 29. Cumulative inactivation of mKv1.7 and mKv1.7-T0. Currents were elicited by a train of 8 depolarizing pulses with a duration of $5 \mathrm{sec}$ to $+40 \mathrm{mV}$ with an interpulse interval of $1 \mathrm{sec}$ from a $V_{\mathrm{h}}$ of $-100 \mathrm{mV}$ (Inset). The current amplitude decreases significantly during this train of pulses from the first pulse (the largest) to the 8th (the smallest). No p/n protocol was applied. Panels A. and B. show eight current traces from mKv1.7 and mKv1.7-T0, respectively. Insets contain first $500 \mathrm{~ms}$ of the consecutive pulses (mKv1.7 wt in black; mKv1.7-T0 in gray) C. Cumulative inactivation time course plotted as the fraction of the current left after each consecutive pulse. Evaluation of cumulative inactivation was made by fitting the fractional current $\left(I_{1 \text { st }}\right.$ pulse $/ I_{n}$ th pulse $)$ vs pulse number plots to a mono-exponential function. mKv1.7 is black $(n=15)$ and $m K v 1.7-T 0$ is gray $(n=12)$.

determined. A rapid decline in the amplitude of the peak currents elicited by successive test pulses from both channels investigated is shown (Fig.29A and B). The currents decreased during trains of stimuli consisting of 8 depolarizations to $+40 \mathrm{mV}$ separated by $1 \mathrm{sec}$ periods at a holding potential of $-100 \mathrm{mV}$ (inset), no $\mathrm{p} / \mathrm{n}$ protocol was implemented for this type of experiments. For this, pulses were long enough (5sec) to achieve a comparable level of inactivation from both, fast and slow inactivating channels (Fig.29A and B). Under these conditions, mKv1.7-T0 showed much slower cumulative inactivation kinetics than mKv1.7 wt. Plots of normalized test pulse current vs pulse number were accurately fit with a mono-exponential decay functions that render values for the quantification of the kinetics of the process. Cumulative inactivation mean time constants $t a u_{\mathrm{Cl}}$ of $22.5 \pm 4.4 \mathrm{sec}$ and $8.14 \pm 0.3 \mathrm{sec}$ for mKv1.7-T0 $(n=20)$ and mKv1.7 wt $(n=23)$ were obtained respectively (Table 7). Accordingly, comparing the time course of cumulative inactivation of wild type and 

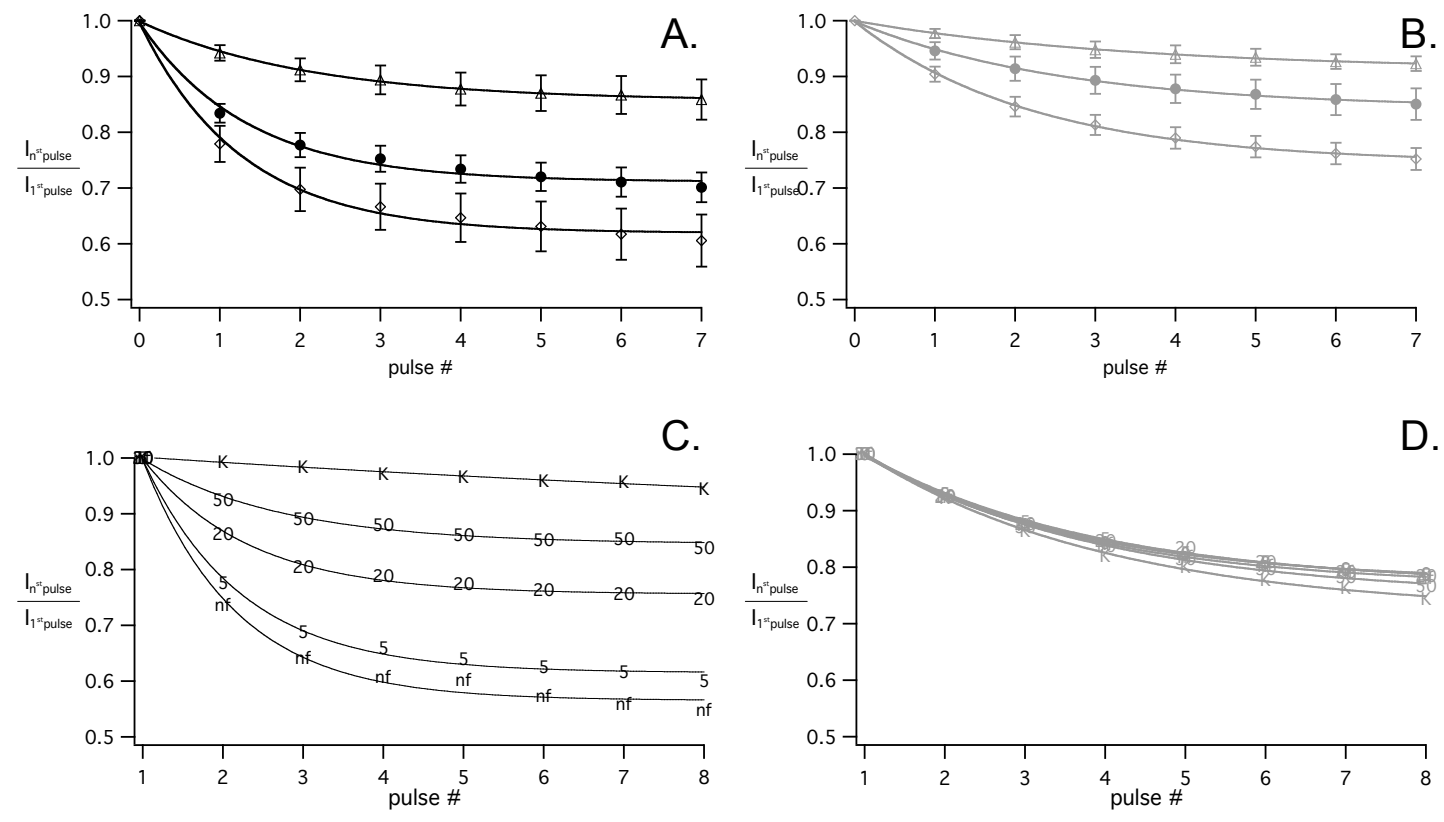

Figure 30. Potassium dependence of cumulative inactivation of mKv1.7 wt and mKv1.7T0. Current were elicited by a train of 8 depolarizing pulses of $5 \mathrm{sec}$ to $+40 \mathrm{mV}$ once every second from a $V_{h}$ of $-100 m V$. Cumulative inactivation time course plotted as the fraction of the current left after each consecutive pulse. Evaluation of cumulative inactivation was made by fitting the fractional current $\left(I_{1 s t}\right.$ pulse $/ I_{n^{\text {th }}}$ pulse) vs pulse number plots to a mono-exponential function. A. mKv1.7 wt fractional currents vs pulse number. Solid symbols are NFR $\left(2.5 \mathrm{mM} \mathrm{K}^{+} / 115 \mathrm{mM} \mathrm{Na}^{+}, \mathrm{n}=12\right)$, triangles are $117.5 \mathrm{mM}$ $\mathrm{K}^{+}\left(0 \mathrm{mM} \mathrm{Na}^{+}, \mathrm{n}=6\right)$, and open rhombi are $117.5 \mathrm{mM} \mathrm{Na}^{+}\left(0 \mathrm{mM} \mathrm{K}^{+}, \mathrm{n}=5\right)$. B. mKv1.7-T0 symbols as in $A(n=12,6$, and 4 , respectively ). C and D. Effect of different concentrations of potassium over cumulative inactivation of mKv1.7 and mKv1.7-T0, respectively. Average values of 4 oocytes per $\mathrm{K}^{+}$concentration $\left(\mathrm{nf}=2.5 \mathrm{mM} \mathrm{K}^{+}, 5,20\right.$, 50 and $\mathrm{K}=117.5$, are $\left[\mathrm{K}^{+}\right]_{0}$ in $\left.\mathrm{mM}\right)$.

mutant mKv1.7-T0 channels it can be seen that mKv1.7 wt inactivates faster than the mutant homolog (Fig.29C).

\subsection{Influence of extracellular potassium on the cumulative inactivation}

The effect of different concentrations of $\mathrm{K}^{+}$on the cumulative inactivation of mKv1.7 wt and mKv1.7-T0 channels was investigated. The cumulative inactivation events observed in control conditions using the pulse trains described previously were used in the absence of $\mathrm{K}^{+}\left(117.5 \mathrm{mM} \mathrm{Na}^{+}\right)$and in presence of increasing concentrations of the ion $\left(2.5\right.$ to $117.5 \mathrm{mM} \mathrm{K}^{+}, \mathrm{Na}^{+}$was added to final $\left[\mathrm{K}^{+}+\mathrm{Na}^{+}\right]$ $=117.5 \mathrm{mM}$ ). Panels $A$ and $B$ of figure 30 show mono-exponential fit plots of the 
cumulative inactivation process under nominal zero $\mathrm{K}^{+}$(empty triangles), control (solid circles), and $\mathrm{K}^{+}$-Ringer (empty rhombi) conditions of Kv1.7 wt and Kv1.7-T0, respectively. The cumulative inactivation in both channel types resulted to some extent sensitive to extreme concentrations of $\mathrm{K}^{+}$(Figures 30A and $\mathrm{B}$ and table 7). However mKv1.7 wt shows a nice graded, and progressive slowing of the cumulative inactivation process with increasing concentrations of potassium, whereas the mutant channels $\operatorname{tau}_{\mathrm{Cl}}$ seemed to be not "potassium dose" sensitive (Figure $30 \mathrm{C}$ and D).

Table 7.

Cumulative Inactivation time constants of mKv1.7 wt and mKv1.7-T0

\begin{tabular}{|c|c|c|c|}
\hline & \multicolumn{3}{|c|}{$\operatorname{tau}_{\mathrm{Cl}}(\mathrm{sec})$} \\
\hline & $0 \mathrm{mM} \mathrm{K}^{+}$ & $2.5 \mathrm{mM} \mathrm{K}^{+}$ & $117.5 \mathrm{mM} \mathrm{K}^{+}$ \\
\hline $\begin{array}{l}\mathrm{mKv} 1.7 \mathrm{wt} \\
\text { mean } \pm \mathrm{sem}\end{array}$ & $\begin{array}{c}7.3 \pm 0.3^{\text {ns }} \\
n=6\end{array}$ & $\begin{array}{c}8.14 \pm 0.3 \\
n=20\end{array}$ & $\begin{array}{c}14.11 \pm 4.8^{*} \\
n=6\end{array}$ \\
\hline \multirow[t]{2}{*}{$\begin{array}{c}\text { mKv1.7-T0 } \\
\text { mean } \pm \text { sem }\end{array}$} & $\begin{array}{c}14.6 \pm 2.1^{\mathrm{ns}} \\
\mathrm{n}=3\end{array}$ & $\begin{array}{c}22.5 \pm 4.4 \\
n=23\end{array}$ & $\begin{array}{c}38.0 \pm 14.6^{\mathrm{ns}} \\
\mathrm{n}=5\end{array}$ \\
\hline & \multicolumn{3}{|c|}{$p=0.0042$} \\
\hline
\end{tabular}

\subsubsection{Recovery from inactivation of mKv1.7 channels}

After inactivation voltage gated ion channels have to undergo a conformational change to be activatable again, this process is regarded as recovery from inactivation. Because wild type and T0 channels had a marked difference in the development of inactivation we decided to determine if the recovery from inactivation was also affected. To study the kinetics parameters a double pulse protocol was used where two depolarizing pulses to $+40 \mathrm{mV}\left(\mathrm{P}_{1}\right.$ and $\left.\mathrm{P}_{2}\right)$ were spaced by increasingly long interpulse intervals at $-100 \mathrm{mV}$. Such stimulation sequence is presented in the inset of Fig.31A, observe that the first pulse, $P_{1}$, was made long enough in order to elicit a profound inactivation from which channels were allowed to recover at $-100 \mathrm{mV}$ where the probability for the channels to be open is extremely low. Scaled and superimposed current traces from mKv1.7 and mKv1.7-T0 (black and gray traces, respectively) channels in response to the double pulse protocol described are shown in the panel $\mathrm{A}$ 
A.

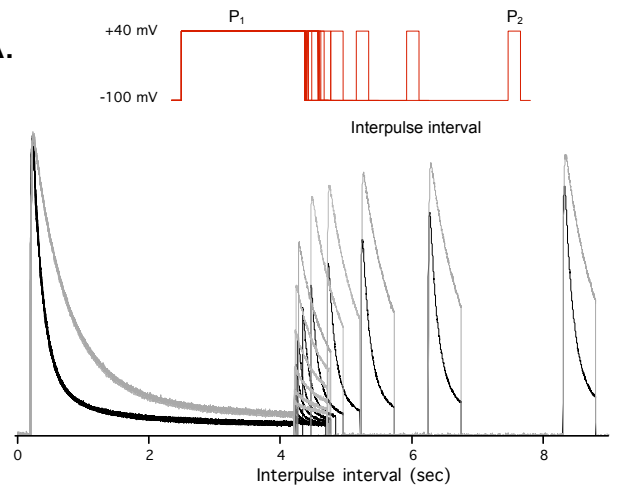

C.

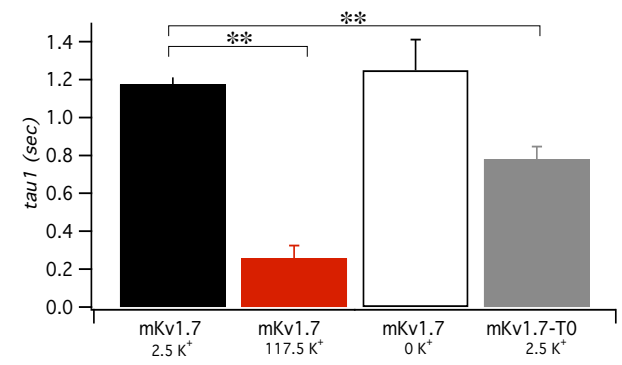

B.

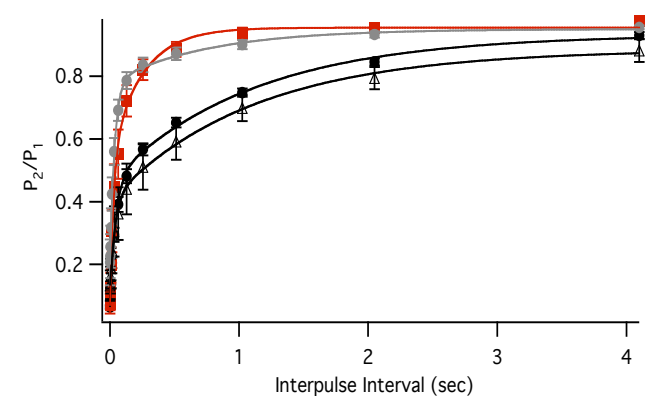

D.

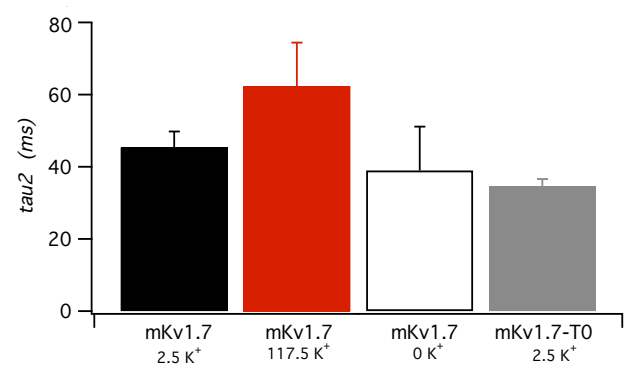

Figure 31. Recovery from inactivation of mKv1.7 wt and mKv1.7-T0 channels. A. Superimposed currents traces from mKv1.7 wt and mKv1.7-T0 channels. The protocol used to examine recovery from inactivation is shown in the inset. Depolarizing pulses $P_{1}$ (4.5sec) and $\mathrm{P}_{2}(500 \mathrm{~ms})$ were to $+40 \mathrm{mV}$ were applied, with repolarization to $-100 \mathrm{mV}$ during the interpulse interval. Each set of stimulation consisting of $P_{1}$, interpulse interval and $P_{2}$ was spaced from next one by $1 \mathrm{~min}$ at $-100 \mathrm{mV}$. B. The time course of recovery from inactivation. The $P_{1} / P_{2}$ ratio is shown as a function of time. Black circles, red squares and empty triangles correspond to $\mathrm{mKv} 1.7$ wt with $0,2.5$, and $117 \mathrm{mM} \mathrm{K}^{+}$in the bath $(n=15,6$, and 6 , respectively). Gray circles show the recovery from inactivation time course of mKv1.7-T0 under control conditions $\left(2.5 \mathrm{mM} \mathrm{K}^{+}\right)$. Time constants for recovery from inactivation were determined by fitting $P_{2} / P_{1}$ ratios vs interpulse interval times to a double exponential function for all the cases presented (smooth lines in B). C and D. Bar diagrams of the calculated time constants tau1 and tau2, obtained from the experiments in $\mathrm{B}$ (mean \pm sem).

of figure $31 \mathrm{E}$. The ratios from $\mathrm{P}_{2} / \mathrm{P}_{1}$ are a measure of the fraction of channels that recovered from inactivation during the interpulse interval at $-100 \mathrm{mV}$, what is presented in the graph offered in the panel B. The time course of the recovery from inactivation studied in this work had a bi-exponential behavior as observed in the $P_{2} / P_{1}$ vs interpulse time of mKv1.t wt and mutant elicited currents suggesting the presence of two distinct processes involved in the inactivation. In agreement to the observed inactivation features of both channels, the recovery resulted faster for the slow inactivating mKv1.7-T0 than for the faster inactivating wild type channel $(0.8 \pm 0.1 \mathrm{sec} v \mathrm{~s}$ $1.2 \pm 0.1 \mathrm{sec}$, respectively, $p=0.002$ ). More striking is the different contributions of the slow and fast components of both channels. Accordingly, the relative amplitude of the slow component in the wild type mKv1.7 was about $54 \%$ while in the T0 mutant the 
contribution of the slow component of the recovery from inactivation was only $20 \%$. In addition, mKv1.7 wt recovers significantly faster in the presence of high $\left[\mathrm{K}^{+}\right]_{0}$. A comparison of the two time constants obtained from fits to the $P_{2} / P_{1}$ ratios is given in panels $\mathrm{C}$ and $\mathrm{D}$ of Fig.31. In $\mathrm{C}$, the first and slower recovery time constant tau1 from mKv1.7 wt channels (black bar) is compared with the slow time constant (tau1) obtained for mKv1.7-T0 currents (gray bar). Furthermore, mKv1.7 wt tau1 for the recovery from inactivation not only got significantly faster with a tau1 $=0.7 \pm 0.2 \mathrm{sec}$ $(p=0.03)$ but also contributed to the overall recovery of the wild type channels by increasing the fast component about $62 \%$ when the channels were exposed to $117.5 \mathrm{mM} \mathrm{K}^{+}$(red bar). The faster time constant obtained for the recovery from inactivation was comparable between the channel types and conditions tested as shown in Fig.31D although in mKv1.7-T0 channels the relative amplitude of the fast component is smaller. Consistently, the absence of the 32 amino-terminal amino acids and the faster recovery kinetics in presence of high extracellular potassium are related to faster recovery from inactivation in mKv1.7 channels. All this results suggesting that the inactivation of mKv1.7 channels is the result of two different mechanisms that can be distinguished by its relevance in the wild type and mutant channels inactivation process.

\subsection{2 hKv1.7}

Sequence homology between the complete sequence of the mouse Kv1.7 channels and the human ortholog reaches $93.2 \%$. Therefore, it was expected to observe the same characteristics in the currents elicited by the human and mouse constructs. It is proper to remind that the start codon AUG, that we believe is the transcription initiation point of the mKv1.7 wt clone, could not be identified in the human sequences neither on the cDNAs cloned by ourselves nor in the ones published in the public data bases. As a consequence we inferred that the highest homology must be observed between the mKv1.7-T0 and the human clones. The characterization of the ortholog Kv1.7 was performed using the two electrodes voltage clamp technique (TEVC). Accordingly, oocytes injected with cRNA encoding for hKv1.7 presented outward currents upon stimulation with the standard IV protocol we used for the characterization of the mouse clones (inset in Fig.21C). Figure 32A shows 
A.

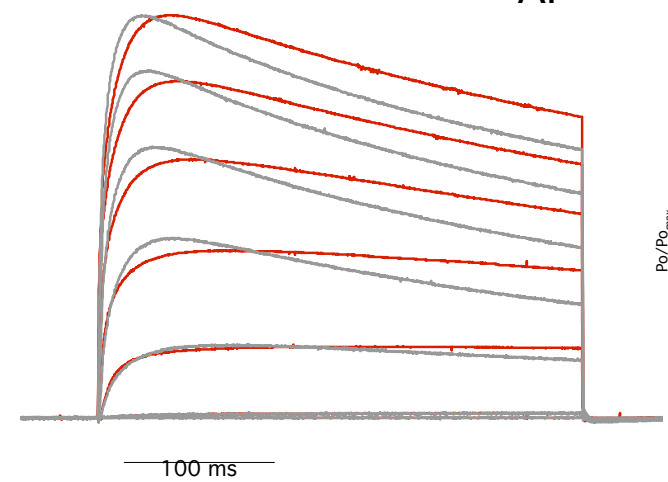

B.

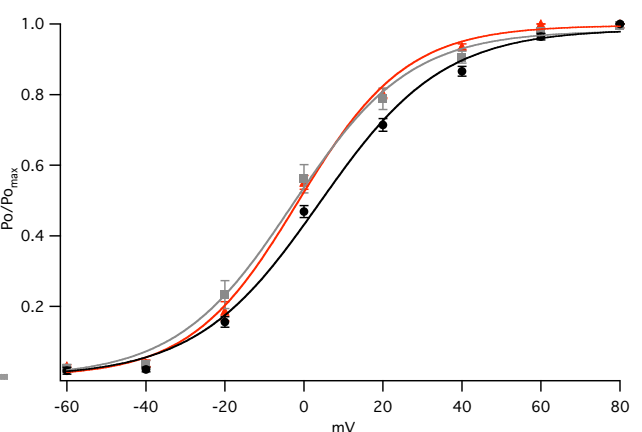

Figure 32. Representative voltage dependent currents from $X$. laevis oocytes expressing hKv1.7 wt and mKv1.7-T0 channels. A. Outward currents in response to $500 \mathrm{~ms}$ depolarizing test potentials ranging from -60 to $+80 \mathrm{mV}$ in $20 \mathrm{mV}$ increments $(15 \mathrm{sec}$ between pulses), $V_{h}=-100 \mathrm{mV}$. hKv1.7 wt is shown in red and mKv1.7-T0 in gray. B. Steady state activation curves for hKv17wt and mKv1.7-T0. The relative open probabilities were plotted against the potential and were fitted to a Boltzmann function (see methods).

representative hKv1.7 currents in an overlay with mKv1.7-T0 current traces. From the currents obtained from hKv1.7 several aspects were investigated. The conductance and relative open probabilities for potentials ranging from -60 to $+80 \mathrm{mV}$ were calculated as detailed in the methods section of this work. The steady state activation curves derived from the collected data on hKv1.7 channels matched satisfactorily the average values obtained for mKv1.7-T0; this is evidenced by the plot in panel B from figure 32. The mean \pm sem values of half activation potential and slope parameters are contained in Table 8 with the corresponding significances resulting from the statistical analysis.

Table 8.

Steady state activation parameters of mKv1.7-T0 and hKv1.7 channels.

mKv1.7-T0 hKv1.7

mean \pm sem mean \pm sem $\quad p$

\begin{tabular}{cccc}
$\mathrm{V}_{1 / 2}$ & $-4.33 \pm 2.9$ & $-2.0 \pm 1.58$ & $0.45^{\mathrm{ns}}$ \\
slope & $13.3 \pm 0.6$ & $12.3 \pm 0.4$ & $0.22^{\text {ns }}$ \\
\hline $\mathrm{n}$ & 15 & 28 & \\
\hline ns: not significant & &
\end{tabular}


A.

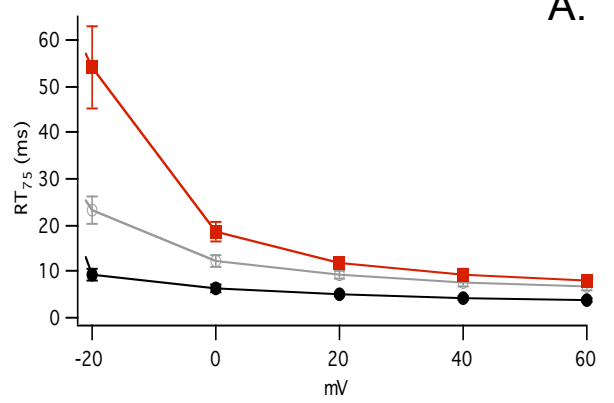

B.

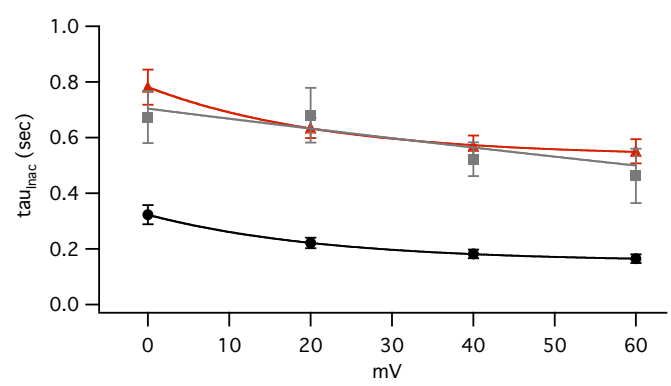

Figure 33. A. Activation kinetics of hKv1.7 and mKv1.7-T0 from TEVC experiments. Rise time determined as the time in ms required to reach $75 \%$ of $I_{\max }(n=20)$. B. Inactivation time constants derived from mono-exponential functions fit to the current decay, tau $u_{\text {Inac }}$, plot of hKv1.7 and mKv1.7-T0 currents ( $\mathrm{n}=20$ and 13, respectively). mKv1.7 wt curves are shown in black for comparison.

\subsubsection{Activation of hKv1.7 channels}

Figure $33 \mathrm{~A}$ contains the plot of the rise times to $75 \%$ peak current of the three channels studied in this work. Table 8 summarizes the $\mathrm{RT}_{75}$ values for mKv1.7-T0 and hKv1.7 activating currents. Currents measured from Xenopus oocytes injected with $\mathrm{hKv1.7}$ wt cRNA reached $75 \%$ of the maximal current at a command potential of $+40 \mathrm{mV}$ in about $9.4 \mathrm{~ms}$, very similar to the $\mathrm{RT}_{75}$ value obtained for mKv1.7-T0 at the same potential $(7.5 \mathrm{~ms}, p=0.23)$; hence evidencing the high similarity in the activation kinetics of these two channels.

Table 9.

Rise times to $75 \%$ of the peak currents of mKv1.7-T0 and hKv1.7 channels.

\begin{tabular}{cccc}
\hline & \multicolumn{3}{c}{$\mathrm{RT}_{75}(\mathrm{~ms})$} \\
\hline & mKv1.7-T0 & $\mathrm{hKv1.7}$ & $\mathrm{p}$ \\
$\mathrm{V}_{\mathrm{m}}$ & mean \pm sem & mean \pm sem & \\
\hline$-20 \mathrm{mV}$ & $23.3 \pm 2.9$ & $54.16 \pm 8.8$ & 0.0135 \\
$0 \mathrm{mV}$ & $12.4 \pm 1.2$ & $18.59 \pm 2.3$ & 0.0529 \\
$20 \mathrm{mV}$ & $9.36 \pm 0.9$ & $11.85 \pm 1.3$ & 0.1929 \\
$40 \mathrm{mV}$ & $7.47 \pm 0.7$ & $9.38 \pm 1.12$ & 0.2269 \\
$60 \mathrm{mV}$ & $6.78 \pm 0.7$ & $7.99 \pm 0.9$ & 0.3811 \\
\hline $\mathrm{n}$ & 12 & 20 &
\end{tabular}




\subsubsection{Inactivation of hKv1.7 channels}

Apparently the currents elicited trough human Kv1.7 channels were slightly slower than the mouse T0 currents, and certainly slower than mKv1.7 wt. However it is proper to remind that we found big variability in the inactivation time constants of all the clones characterized in this work. Therefore we emphasize that the inactivation process in Kv1.7 bears two tendencies, a fast mode exemplified by mKv1.7 wt channels and the slow mode evidenced in mKv1.7-T0 and hKv1.7 wt channels. The inactivation of hKv1.7 currents followed a shallow and monotonic pattern throughout the range of voltages to which the channels were challenged (Fig.33B). Average inactivation time constants $t_{\text {Inac }}$ peaked $(781.2 \pm 63 \mathrm{~ms})$ at mild depolarizing pulses $(0 \mathrm{mV})$, and found a minimum at strong depolarizations to $+60 \mathrm{mV}$ (550.6 $\pm 44 \mathrm{~ms})$ (Table 10, Fig.33). Furthermore, no significant differences were detected through the statistical comparison of the quantitative data concerning the inactivation process of the human channel elicited currents and mKv1.7-T0. The human Kv1.7 channel revealed an evident parallelism with mouse T0 channels as stated in Table 10 (Figure 33B).

Table 10.

Inactivation time constants tau $u_{\text {Inac }}$ of mKv1.7-T0 and hKv1.7.

\begin{tabular}{cccc}
\hline \multicolumn{3}{c}{$\operatorname{tau}_{\text {Inac }}(\mathrm{ms})$} \\
\hline & mKv1.7-T0 & hKv1.7 & $\mathrm{p}$ \\
$\mathrm{V}_{\mathrm{m}}$ & mean \pm sem & mean \pm sem & \\
\hline $0 \mathrm{mV}$ & $767.5 \pm 116.9$ & $781.2 \pm 62.9$ & $0.9113^{\mathrm{ns}}$ \\
$20 \mathrm{mV}$ & $877.4 \pm 144.0$ & $634.8 \pm 36.2$ & $0.0587^{\mathrm{ns}}$ \\
$40 \mathrm{mV}$ & $643.2 \pm 80.1$ & $569.2 \pm 38.3$ & $0.3614^{\mathrm{ns}}$ \\
$60 \mathrm{mV}$ & $560.1 \pm 51.5$ & $550.6 \pm 43.5$ & $0.890^{\mathrm{ns}}$ \\
\hline $\mathrm{n}$ & 13 & 20 & \\
\hline
\end{tabular}

ns: not significant 
E.

F.
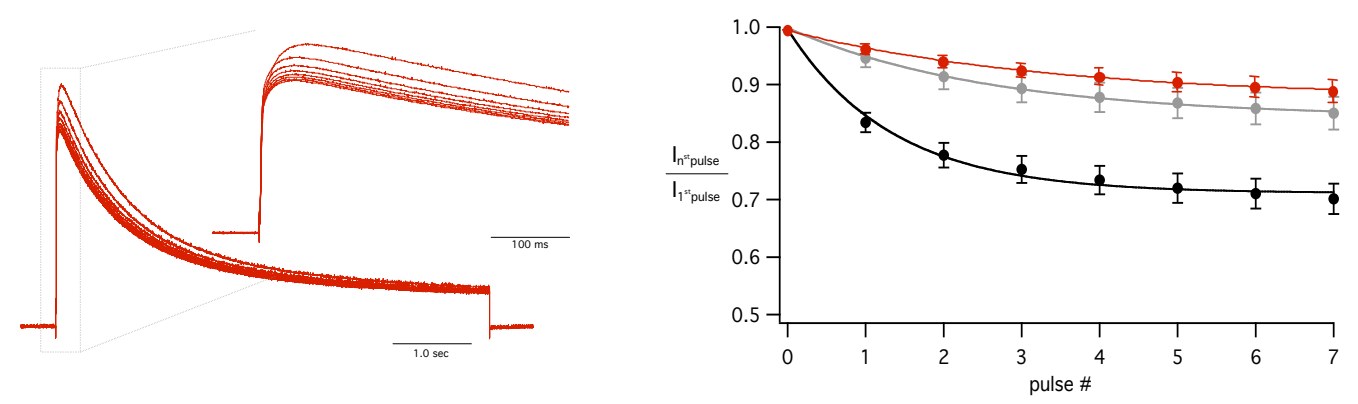

Figure 34. Cumulative inactivation of hKv1.7, similarities to mKv1.7-T0. A. Current traces from oocytes expressing hKv1.7 channels subjected to a train of 8 pulses of $5 \mathrm{sec}$ at $0.16 \mathrm{~Hz}$ are shown in red, no $\mathrm{p} / \mathrm{n}$ protocol was applied. B. Cumulative inactivation time course plotted as percentage of the current left after each consecutive pulse. Evaluation of cumulative inactivation was made by fitting the fractional current $\left(I_{1}^{\text {st }}\right.$ pulse $/ l_{n}^{\text {th }}$ pulse) versus pulse number plots, to a mono-exponential function $(n=5)$.

\subsubsection{Cumulative inactivation of hKv1.7 channels}

Due to the fact that, so far, all the characteristics of mKv1.7-T0 channels were paralleled by the human Kv1.7 currents, cumulative inactivation was also assessed. The train of pulse used to uncover the cumulative inactivation phenomenon of the mouse channels (legend and inset in Fig.29) was used to prove that, likewise the mouse channels, the human Kv1.7 channels observed cumulative inactivation as shown in figure $34 \mathrm{~A}$, with a time constant of $20.5 \pm 2.2 \mathrm{sec}$, quite similar to the $\operatorname{tau}_{\mathrm{Cl}}$ of 22.5 $\pm 4.4 \mathrm{sec}$ obtained for the mKv1.7-T0 channels (Fig.34B).

\subsubsection{Recovery from inactivation of $\mathrm{hKv} 1.7$ channels}

Human Kv1.7 currents were challenged to the double pulse protocol designed to study the recovery from inactivation kinetics of $\mathrm{mKv} 1.7$ channels. The plot in the panel B from Fig.35 contains superimposed current traces of experiments in response to the double pulse protocol designed to evaluate the recovery from inactivation. The blue traces correspond to recordings under control, and $50 \mathrm{mM} \mathrm{K}^{+}$ external concentration are represented in red. Although not quantified, from those plots it is obvious that potassium speeds the process of inactivation as has been reported before in this work for mKv1.7-T0 channels. 
A.

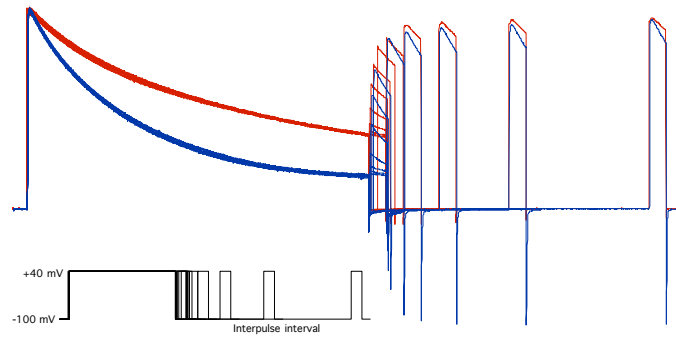

B.

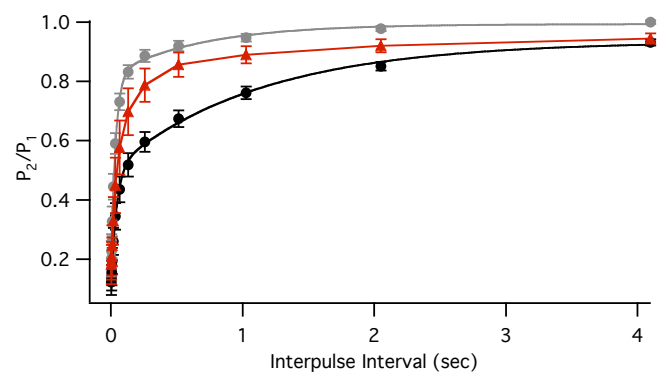

Figure 35. Recovery from inactivation of hKv1.7, similarities to mKv1.7-T0. A. Recovery from inactivation stimulation protocol elicited currents. Scaled and superimposed current traces from $\mathrm{hKv} 1.7$ channels exposed to control conditions $\left(2.5 \mathrm{mM} \mathrm{K}^{+} / 115 \mathrm{mM} \mathrm{Na}^{+}\right.$, red traces) and $50 \mathrm{mM} \mathrm{K}^{+}\left(67.5 \mathrm{mM} \mathrm{Na}^{+}\right.$, blue traces). The protocol used to examine recovery from inactivation is given in the inset. $P_{1}(4.5 \mathrm{sec})$ and $P_{2}(500 \mathrm{~ms})$ were to $+40 \mathrm{mV}$, with repolarization to $-100 \mathrm{mV}$ during the interpulse interval, each set of stimulation consisting of $P_{1}$, interpulse interval, and $P_{2}$ was spaced from the next one by $1 \mathrm{~min}$ at $-100 \mathrm{mV}$. B. The recovery from inactivation time course fitted to a biexponential function. The red smooth line and symbols correspond to average data from 4 oocytes expressing hKv1.7 channels. $\mathrm{mKv1.7} \mathrm{wt}$ and mKv1.7-T0 are shown for comparison in black and gray, respectively.

Finally, the recovery from inactivation followed, as expected, a bi-exponential time course with recovery from inactivation time constants for control conditions $\left(2.5 \mathrm{mK}^{+}\right)$of tau1 $\sim 600 \mathrm{~ms}$ and tau2 of $60 \mathrm{~ms}$, indistinguishable and in perfect agreement with the observations of the mKv1.7-T0 currents (the red trace in figure 35B corresponds to hKv1.7).

\subsection{Pharmacology of Kv1.7 channels}

To establish the pharmacological profile of Kv1.7 cannels the Xenopus laevis oocyte heterologous expression system and TEVC recordings were used to assay the effects of substances known to interact with $\mathrm{K}^{+}$channels. Specially conus snail toxins were assayed on the Kv1.7 channels cloned in this work. This work focused mainly on the reduction of the amplitudes of peak currents referred as current block. However, some kinetic modifications induced by the blockers were addressed. 
A.

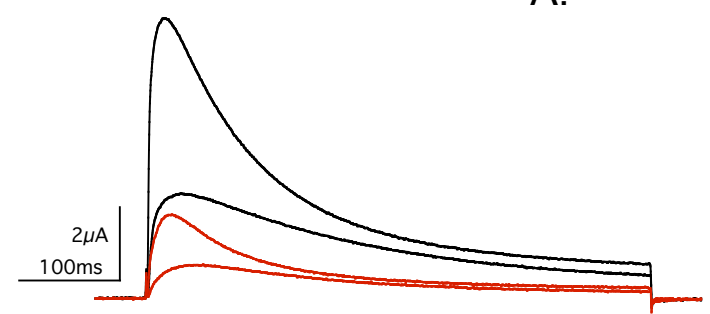

B.

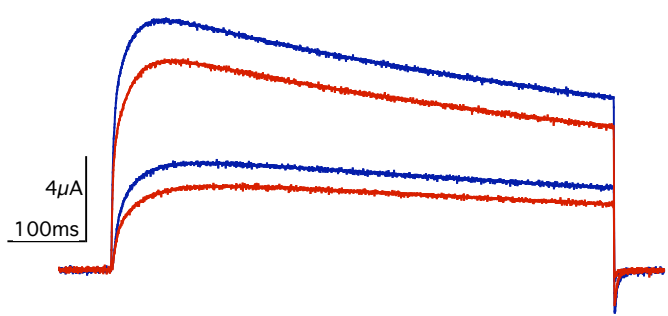

Figure 36. TEA block of Kv1.7 currents. A. and B. mKv1.7 wt and hKv1.7 wt current traces in response to stimulation pulses to 0 and $+40 \mathrm{mV}$. Control conditions (NFR, $2.5 \mathrm{mM} \mathrm{K}^{+}$, $115 \mathrm{mM} \mathrm{Na}^{+}$) are shown in black and blue respectively. Red traces represent currents at the same potential upon application of $0.5 \mathrm{mM}$ TEA to the external bath $\left(2.5 \mathrm{mM} \mathrm{K}^{+}, 114.5 \mathrm{mM}\right.$ $\mathrm{Na}^{+}$).

\subsubsection{Sensitivity to TEA}

The quaternary ammonium ion tetraethyl ammonium (TEA) is a classical $\mathrm{K}^{+}$ channel blocker known to interact with the ion channel pore by occluding the ion pathway with very fast block/unblock kinetics. Application to the bath of TEA resulted in a decrease of the current amplitude. Figure 36A and B show current traces from mKv1.7 wt channels and hKv1.7 channels (respectively) in control conditions and upon application of TEA to the external medium. As it can be appreciated from the figure, TEA showed different affinities for mouse and human channels. The mean $\mathrm{IC}_{50}$ calculated for mKv1.7 wt at $+40 \mathrm{mV}$ was $124 \pm 2 \mu \mathrm{M}$ while for the human channel $\mathrm{IC}_{50}$ for the TEA block was $2.4 \pm 1.4 \mathrm{mM}$ in the same potential; resulting in about 20 times higher affinity of the blocker for the mouse than for the human channels. Furthermore, it has been widely documented that the presence of TEA in the extracellular mouth of the permeation pathway of potassium channels not only blocks the outward currents but also impedes the slow inactivation of the channels (Choi et al., 1991; Labarca \& MacKinnon, 1992).

Mouse and human Kv1.7 wild type mediated currents were exposed to $0.5 \mathrm{mM}$ TEA $\left(2.5 \mathrm{mM} \mathrm{K}^{+}, 115 \mathrm{mM} \mathrm{Na}^{+}\right)$from the extracellular side and the influence of the blocker on the inactivation kinetics was studied. Figure 37 comprises scaled current traces of mKv1.7 wt in panel $A$ and hKv1.7 channels in panel $C$, under control and in the presence of TEA. TEA exerted and inhibitory effect on the inactivation of mKv1.7 wt, while not having any evident effect on the current kinetics of the human homolog (Figure 37, and inset). 
A.

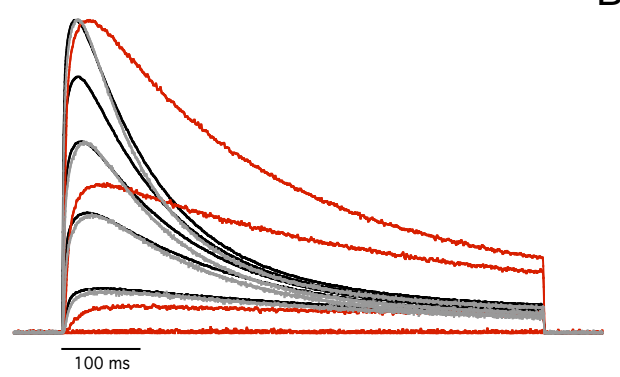

C.

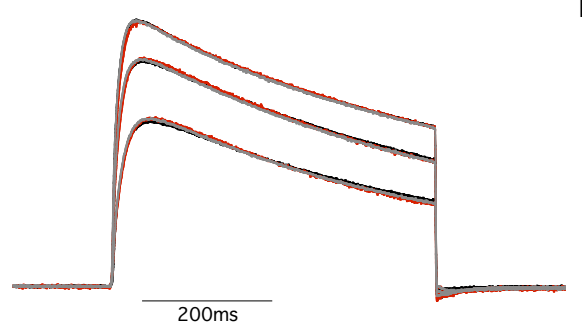

B.

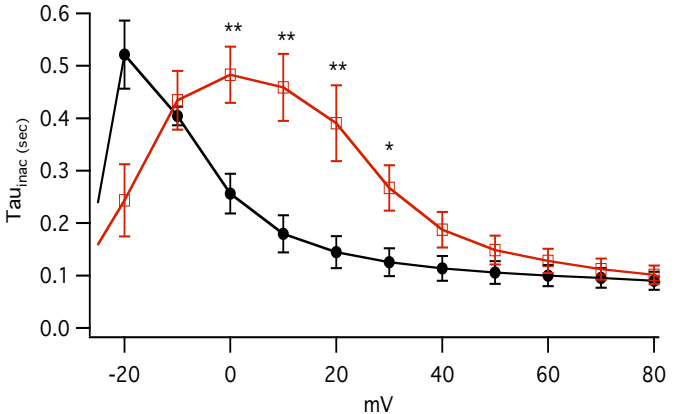

D.

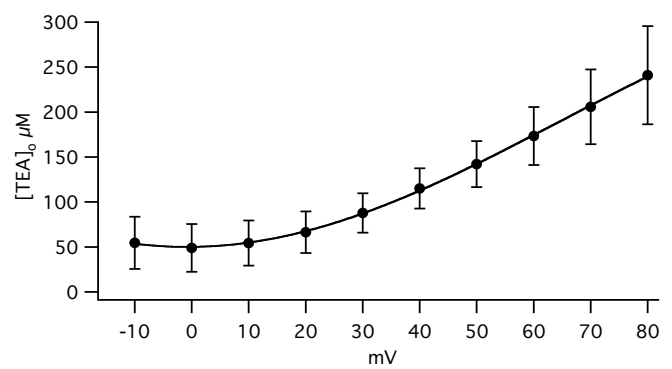

Figure 37. Influence of externally applied TEA on the inactivation kinetics of mKv1.7 wt. A. Scaled mKv1.7 wt currents under control conditions $\left(2.5 \mathrm{mM} \mathrm{K}^{+} / 115 \mathrm{mM} \mathrm{Na}^{+}\right.$, black) and $0.5 \mathrm{mM} \mathrm{TEA}_{\circ}(2.5 \mathrm{mM}$ $\mathrm{K}^{+} / 114.5 \mathrm{mM} \mathrm{Na}^{+}$, red traces), wash with $2.5 \mathrm{mM} \mathrm{K}^{+}$is shown in gray. In the inset same type of experiment but with hKv1.7 mediated currents B. Inactivation time constants derived from monoexponential fits to the current decay during the inactivation process, $\operatorname{ta}_{\text {inac }}$. Values correspond to mean \pm sem of 6 cells. D. Plot of the voltage dependence of the TEA affinity to mKv1.7 wt. IC $\mathrm{C}_{50}$ were calculated as stated in methods, mean \pm sem of 8 experiments are in the ordinate, and the command potential in the abscissa.

The panel $B$ in figure 37 represents the quantification of the effect of TEA in the mouse wild type channel Kv1.7. When exposed to TEA, inactivation of mKv1.7 wt channels was significantly retarded within intermediate potentials, i.e. between 0 to $+30 \mathrm{mV}$. The panel $\mathrm{D}$ contains the plot of the voltage dependence of the affinity of TEA for mKv1.7 wt channels, expressed as $\mathrm{IC}_{50}$ vs command potential. With the $500 \mathrm{~ms}$ pulses used, currents at the voltages tested did not reach steady state, consequently, the later inactivation faces were not estimated, more so in the presence of TEA. It is evident that the affinity of the blocker decreases with progressively stronger depolarizations, suggesting that the blocker is released from it's binding site by the flux of ions or that there most be state dependence of the block where TEA has higher affinity for partially activated states than for the fully activated.

The quaternary ammonium compound 4-aminopyridine is often used in the pharmacological characterization of Kv channels. We could not observe a significant block with a solution of $20 \mathrm{mM} 4-\mathrm{AP}$ in any on the Kv1.7 channels studied. 


\subsubsection{Zinc}

Zinc is known to selectively block delayed rectifier potassium channels and calcium activated potassium channels at the frog motor nerve endings (Miralles et al., 1994). Block of cardiac ATP-sensitive $\mathrm{K}^{+}$channels in the micromolar range has been reported. Accordingly, the $\mathrm{Zn}^{2+}$ block of the mammalian $\mathrm{Kv1.7}$ channels was studied. Figure 38 illustrates the effects of $\mathrm{Zn}^{2+}$ on Kv1.7 currents. Shown superimposed in panels $A, B$ and $C$ are typical control and blocked currents evoked from a holding potential of $-100 \mathrm{mV}$. The voltage protocol consisted of two of $500 \mathrm{~ms}$ depolarizing pulses to 0 and $+40 \mathrm{mV}$. After switching to the $\mathrm{Zn}^{2+}$-containing medium (red traces in the three panels), the pulse currents showed a prominent decrease in the amplitude. In figure 38A the control and treated responses from mKv1.7 wt evoked currents are superimposed before (black traces) and after treatment with $1 \mathrm{mM} \mathrm{Zn}^{2+}$ (red traces). Complete reversal of all of the effects of $\mathrm{Zn}^{2+}$ was not always observed after returning to $\mathrm{Zn}^{2+}$-free medium (not shown). The effect of $2 \mathrm{mM}$ Zinc on mKv1.7-T0 currents is

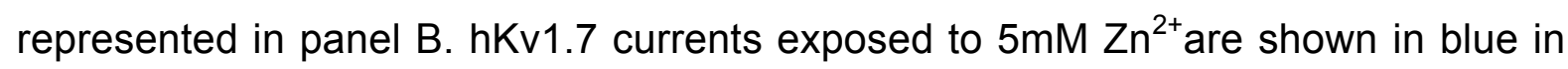
panel C.

A

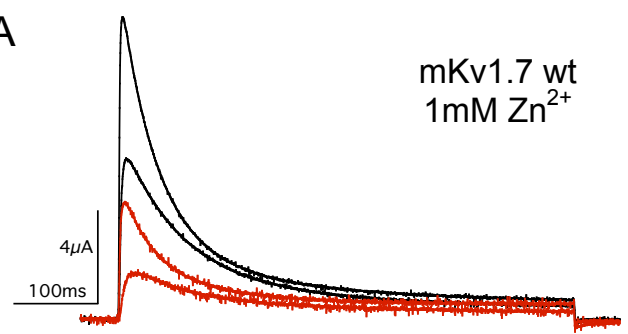

B.

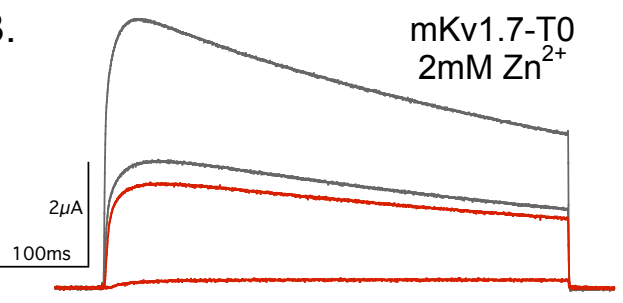

C.

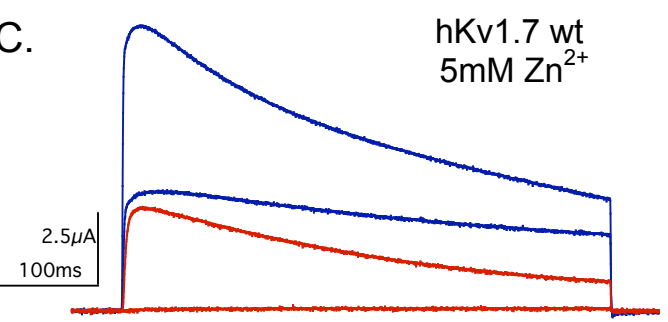

Figure 38. Block of Kv1.7 channels by extracellularly applied Zinc. Current traces in response to depolarizing $500 \mathrm{~ms}$ pulses to 0 and $+40 \mathrm{mV}$. A. mKv1.7 wt currents in control (black) and in the presence of $1 \mathrm{mM} \mathrm{Zn}^{2+}$ (red). B. $\mathrm{mKv} 1.7-\mathrm{T0}$ currents in control are gray and $2 \mathrm{mM}$ $\mathrm{Zn}^{2+}$ are red. C. hKv1.7 currents under control are designated in blue; currents with $\mathrm{Zn}^{+2}$ at a final concentration of $5 \mathrm{mM}$ are red. 


\subsection{3 [M-Conotoxin RIIIK}

$\square \mathrm{M}-\mathrm{R} I \mathrm{IIK}$ is the first peptide cloned from a new family of conotoxins interacting with $\mathrm{K}^{+}$channel. It was cloned from the venom of the marine cone snail Conus radiatus $\square \mathrm{M}-\mathrm{R}$ IIIK; it is known to block Shaker channels with a steady state affinity of about 1 $\square \mathrm{M}$ and with higher potency a trout homolog of Shaker TSha1 ( 20nM). The only mammalian channel that has been reported sensitive to $\square \mathrm{M}-\mathrm{R}$ IIIK is $\mathrm{Kv1.2}\left(\mathrm{K}_{\mathrm{D}}\right.$ 230nM, closed state; Ferber et al. 2004). Figure 39 shows three panels of current traces corresponding to currents elicited by depolarizing pulses to 0 and $+40 \mathrm{mV}$ of channels mKv1.7 wt, mKv1.7-T0, and hKv1.7. All three channels were exposed to different concentrations of $\square \mathrm{M}-\mathrm{R} I I \mathrm{KK}$ and the reduction on the peak current used for the calculation of the $\mathrm{IC}_{50}$. Panel $\mathrm{A}$ in figure 39 shows example recordings of $\mathrm{mKv} 1.7$ wt currents under control and after addition of $167 \mathrm{nM} \square \mathrm{M}-\mathrm{R} I I \mathrm{KK}$ to the bath. The mean $I C_{50}$ calculated for 8 experiments returned a value of $146 \pm 24 n M \square M-R I I I K$. Panel B shows a similar experiment performed with mKv1.7-T0 channels. In this experiment $100 \mathrm{nM}$ of the conotoxin was applied to the extracellular medium. The block of both mouse channels return similar values as expected from identical pore

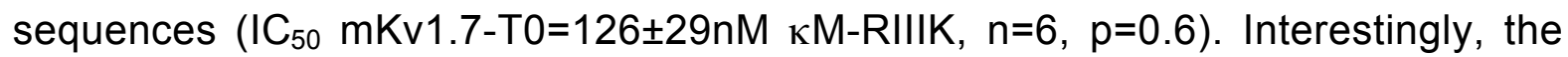
affinity for the human $\mathrm{Kv1.7}$ channels resulted slightly lower with a mean $\mathrm{IC}_{50}$ of $822 \pm 162 n M$ for the block of $\square$ M-RIIIK.

A.

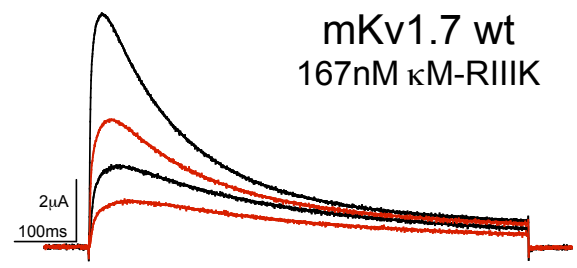

B.

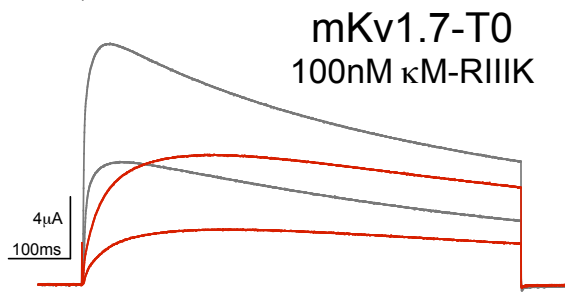

hKv1.7 wt

C.

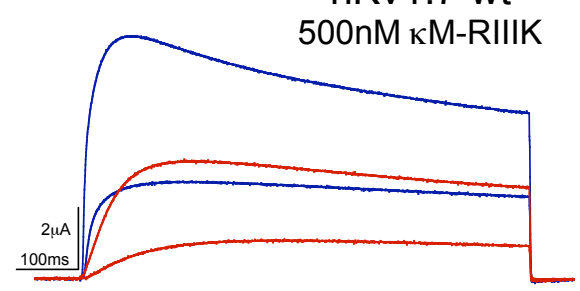

Figure 39. Block of Kv1.7 channels by $\square \mathrm{M}$ conotoxin RIIIK. Current traces in response to depolarizing $500 \mathrm{~ms}$ pulses to 0 and $+40 \mathrm{mV}$. A. mKv1.7 wt currents in control (black) and in the presence of 167nM पM-RIIIK (red). B. mKv1.7-T0 currents in control are gray and $100 \mathrm{nM}$ toxin are red. C. hKv1.7 currents under control are designated in blue; currents with $\square \mathrm{M}-\mathrm{R} I I \mathrm{KK}$ to a final concentration of $500 \mathrm{nM}$ are red. 


\subsubsection{Conkunitzin-S}

An unusually long peptide (more than 60 amino acids) isolated from the venom of Conus striatus that has been proven to block Shaker mediated currents (unpublished results) was tested against the mammalian Kv1.7 channels cloned in this work. Due to an ongoing patent application more details on the sequence and structure of the toxin can not be provided here. Figure 40 shows current traces elicited by 0 and $+40 \mathrm{mV}$ pulses under control (NFR) and upon exposure to 20nM Concunitzin-S (denoted with the red traces). Panel A corresponds to mouse wild type channels elicited currents, control is given in black. In this experiment 20nM Conkunitzin-S generated a decrease in the currents of around 55\%. Mouse mutant channels mKv1.7-T0 displayed currents in panel B showed an average peak current block with an affinity of 94nM. Furthermore, 20nM Concunitzin application to hKv1.7 channels can be seen in the panel C of the figure 40, control conditions are distinguished with blue colored current traces. The human ortholog channel hKv1.7 showed the highest affinity for the block of Conkunitzin-S with a mean affinity of $37 \pm 5 \mathrm{nM}$ given by the $\mathrm{IC}_{50}$ calculation over the diminishing effect of the toxin on the peak current amplitude.

A.

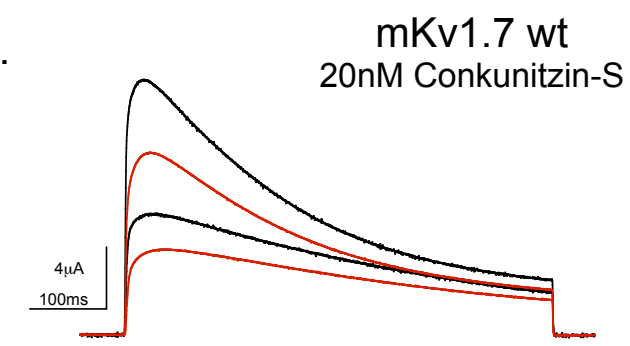

B.

mKv1.7-T0

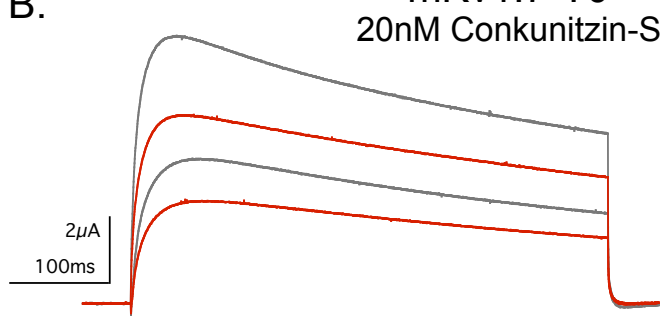

C.

hKv1.7 wt

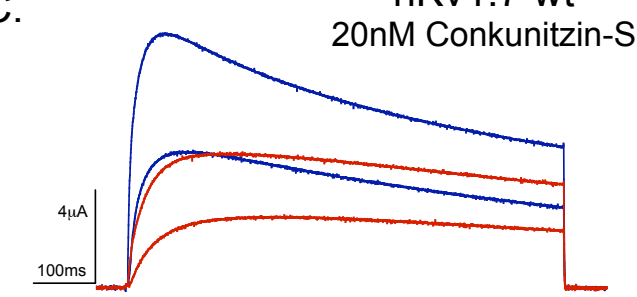

Figure 40. Block of Kv1.7 channels by extracellularly applied Conkunitzin-S. Current traces in response to depolarizing $500 \mathrm{~ms}$ pulses to 0 and $+40 \mathrm{mV}$. All three channels were exposed to 20nM Conkunitzin-S. A. mKv1.7 wt currents in control (black) and under toxin (red). B. mKv1.7-T0 currents in control are gray and Conkunitzin-S are red. C. hKv1.7 currents in control conditions are designated in blue; currents under Conkunitzin-S are red. 


\subsubsection{Redox sensitivity}

The finding that the inactivation of $\mathrm{mKv} 1.7 \mathrm{wt}$ currents varied from the different experiments and the observation that currents were faster in inside out patches prompted the question of the potential mechanism implicated. The evident hypothesis was based on the possible existence of an intracellular "modulator" that was washed away during patch excision. Such modulator could have almost any identity, however other ion channels have been proven to be sensitive to modulation by the oxidative state of the medium not only in an enhancing or suppressing of the current fashion but also modulating the kinetics of the currents. Inside out patches are exposed to the environmental $\mathrm{O}_{2}$ and $\mathrm{CO}_{2}$ concentration (in equilibrium with the bath solution) in contrast to the cytoplasmic physiologically controlled redox conditions that the on cell recordings imply. As a consequence we decided to use the TEVC approach in the evaluation of the influence that the reducing and oxidizing agents respectively, dithiotreithol (DTT) and dithiodipyridine (DTDP) had on Kv1.7 currents. Exposure of oocytes expressing mKv1.7 wt channels to $2 \mathrm{mM}$ DTT led to suppression of the currents. This reduction in current would correspond to a mean $\mathrm{IC}_{50}$ value of $544 \mu \mathrm{M}$ but it did not have significant effects on the currents kinetics (data not shown). However, addition of $200 \mu \mathrm{M}$ of the oxidative chemical DTDP, not only decreased the peak current amplitude with an $\mathrm{IC}_{50}$ of $229 \mu \mathrm{M}$ but it speeded the process of inactivation about 2 fold.

Figure 41 compiles the results of the experiments performed with the redox modifying chemicals DTT and DTDP over Kv1.7 channels; during the whole recording the chamber was continuously perfused with fresh medium (all solutions tested). In panel A currents from mKv1.7 wt under control conditions are shown in black (stimulation pulse to $+40 \mathrm{mV}, \mathrm{V}_{\mathrm{h}}=-100 \mathrm{mV}$ ). The addition to the bath of $200 \mu \mathrm{M}$ DTDP is designated by the red trace, washes with $2 \mathrm{mM}$ DTT and NFR are depicted in gray and green, respectively. The scaled and superimposed traces presented in Fig.41B correspond to the experiment in A. There it is evident the acceleration of the inactivation effect that DTDP produced over mKv1.7 wt currents. Wash with DTT results in partial recovery of the inactivation kinetics observed under control conditions. Further wash with NFR completely rescued the inactivation kinetics although the peak current amplitude could not return to the control level. Panel $\mathrm{C}$ from figure 41 shows experiments with mKv1.7-T0 and hKv1.7 exposed to DTDP in 
A.

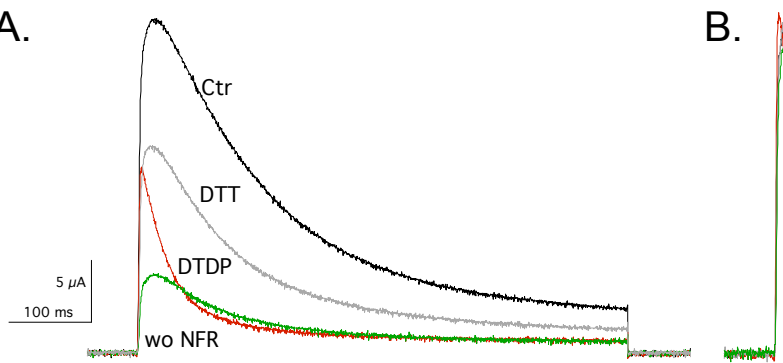

B.

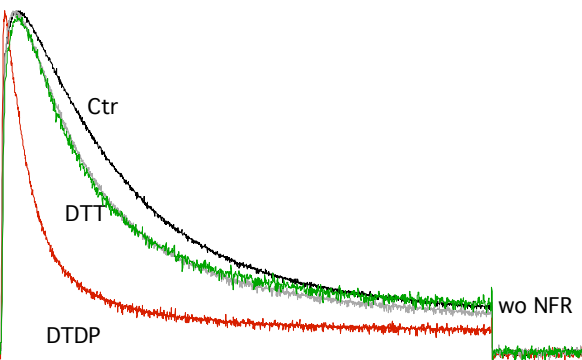

C.
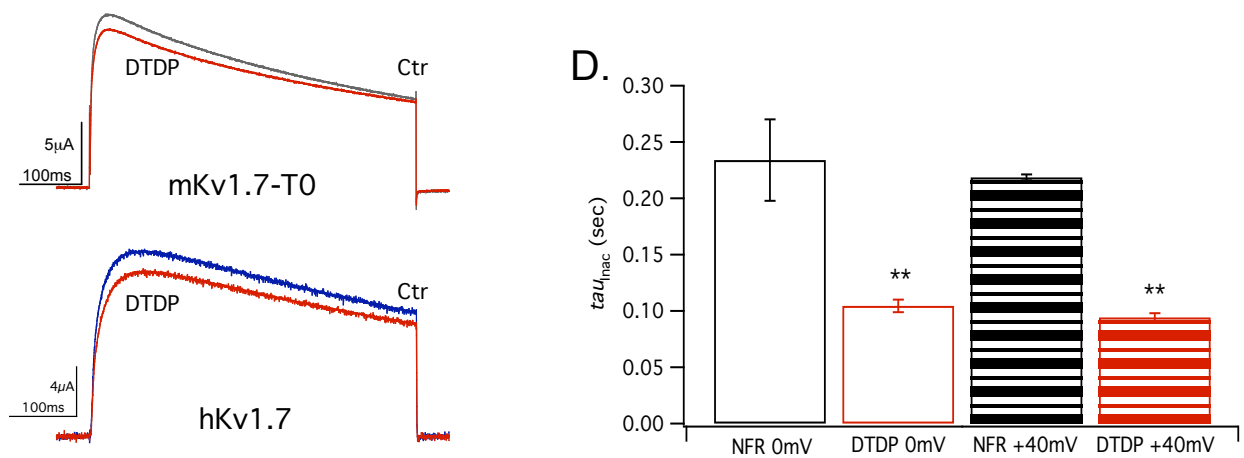

Figure 41. Effect of the oxidizing agent DTDP over Kv1.7 currents. A. Current traces from a Xenopus oocyte injected with cRNA encoding for murine Kv1.7 wild type channels. In black is shown a current trace recorded upon a depolarizing pulse to $+40 \mathrm{mV}, \mathrm{V}_{\mathrm{h}}=-100 \mathrm{mV}$. The

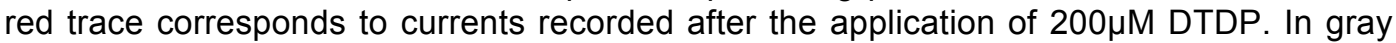
and green the washes with $2 \mathrm{mM}$ DTT and NFR, respectively. B. Scaled and superimposed traces for the experiment in A (colors as in A.). C. mKv1.7-T0 and hKv1.7 currents from $+40 \mathrm{mV}$ pulses in control conditions are gray and blue. Currents with 200 $\mathrm{M}$ DTDP in the bath medium are shown in red. D. Bar diagram of the inactivation time constants obtained from fits to the current decay from pulses to 0 and $+40 \mathrm{mV}$ (black is control, red is $200 \mu \mathrm{M}$ DTDP; $n=6)$.

the same conditions as in A. As shown in Fig.41C the presence of DTDP in the bath resulted in a mild decrease of the mKv1.7-T0 and hKv1.7 channel currents. Complete wash of DTT and DTDP effects were not achieved due to the fact that both chemicals are membrane permeable and therefore not reachable in TEVC experiments.

Accordingly, the quantification of the acceleration of the inactivation process of the mKv1.7 wt currents was assessed as described before by fitting the current decay to a single exponential function. The $t a u_{\text {Inac }}$ obtained are compared in figure 41D. The results are given for the evaluation of currents elicited by pulses to 0 and $+40 \mathrm{mV}$ in order to address both partially and fully activated states. From this bar diagram the highly significant reduction of the inactivation time constants in the presence of DTDP can be seen directly $\left(\operatorname{tau}_{\text {Inac }} 218 \pm 3 \mathrm{~ms}\right.$ vs $94 \pm 4 \mathrm{~ms}$ at $+40 \mathrm{mV}$; in control and DTDP, respectively). 
A summary of the pharmacological data is shown in table 11 .

Table 11.

Pharmacology of Kv1.7 channels

\begin{tabular}{|c|c|c|c|c|}
\hline \multicolumn{5}{|c|}{ Pharmacology } \\
\hline & $\begin{array}{l}\mathrm{mKv} 1.7 \mathrm{wt} \\
\text { mean } \pm \text { sem }\end{array}$ & $\begin{array}{l}\mathrm{mKv} 1.7-\mathrm{T0} \\
\text { mean } \pm \text { sem }\end{array}$ & $\begin{array}{l}\mathrm{hKv} 1.7 \mathrm{wt} \\
\text { mean } \pm \text { sem } \\
\end{array}$ & $p$ \\
\hline TEA & $0.124 \pm 0.2$ & 2.74 & $2.4 \pm 1.4$ & 0.0325 \\
\hline $\mathrm{mM}$ & $n=9$ & $n=1$ & $n=4$ & \\
\hline Zinc & $0.43 \pm 0.002$ & $1.9 \pm 0.6$ & 2.32 & \\
\hline $\mathrm{mM}$ & $n=3$ & $n=2$ & $n=1$ & \\
\hline पM-RIIIK & $146 \pm 24$ & $126 \pm 29^{\text {ns }}$ & $822 \pm 162$ & 0.0003 \\
\hline $\mathrm{nM}$ & $\mathrm{n}=8$ & $\mathrm{n}=6$ & $n=5$ & \\
\hline Concunitzin & $115 \pm 66$ & $94 \pm 43$ & $37 \pm 5$ & 0.0175 \\
\hline $\mathrm{nM}$ & $\mathrm{n}=2$ & $n=3$ & $n=6$ & \\
\hline DTDP & $229 \pm 52$ & ND & $1280 \pm 152$ & 0.0001 \\
\hline$\square \mathrm{M}$ & $n=6$ & & $\mathrm{n}=6$ & \\
\hline DTT & $0.54 \pm 0.1$ & ND & ND & \\
\hline $\mathrm{mM}$ & $\mathrm{n}=6$ & & & \\
\hline
\end{tabular}

$\mathrm{IC}_{50}$ values calculated from peak currents at $+40 \mathrm{mV}$.

Significance is given for the comparison between shaded cells.

ns: not significant when compared to mKv1.7 wt. 


\section{DISCUSSION}

There has been confusing or incomplete data regarding the sequence, the biophysical characteristics and the pharmacology of Kv1.7 channels. The data presented in this work differs from the reports of Kalman et al. (1998) and Bardien et al. (2002); which are the only published information to date on Kv1.7 channels. We will focus first on the analysis of the data generated by us to set the premises for the discussion of the findings of other authors.

\subsection{Cloning of Kv1.7 channels}

In the present study we have cloned, expressed and characterized both mouse and human cDNAs encoding for the proteins mKv1.7 and hKv1.7 voltage activated $\mathrm{K}^{+}$channels.

The sequence of the mouse kcna7 gene has $70 \%$ GC content in the 5 ' end as well as a high sequence complementarity as depicted in the RNA secondary structure plot in figure 12. The high GC content implies the existence of regions of high homology that not only burden the design of appropriate primers but also are the source of RNA self-pairing, hence secondary structure formation, with the additional "tightness" of the triple bond formed between GC pairs. The stability of the complex secondary structure in the RNA expressed as the free energy of the RNA molecule in the case of kcna7 ranged from $-274 \mathrm{Kcal} / \mathrm{mol}$ at $37^{\circ} \mathrm{C}$ to $-142 \mathrm{Kcal} / \mathrm{mol}$ at $65^{\circ} \mathrm{C}$. Therefore, because RNA reverse transcription requires prossessive DNA synthesis by reverse transcriptase, secondary structures frequently interrupt the RNA templates, causing early termination during the synthesis of cDNA and therefrom difficulties in full-length cDNA synthesis. As a consequence the amplification of kcna7 cDNA resulted laborious and difficult, being only possible by addition of saturating concentrations of co-solvents to destabilize the secondary structure of the mRNA for the reverse transcription to proceed. 
Additionally, the $410 \mathrm{bp}$ in front of the AUG (positions -410 to -1 ), presumably corresponding to the promoter of kcna7 have $\square \mathrm{G}$ of $-94.7 \mathrm{Kcal} / \mathrm{mol}$. It has been established that sequences with free energies bigger than $-61 \mathrm{Kcal} / \mathrm{mol}$ in the $5^{\prime}$ untranslated region of eukaryotic RNAs could inhibit efficiently the translation by blocking the formation of the initiation complex (Pelletier \& Sonenberg, 1985; Kozak, 1989). Suggesting that the transcription of kcna7 could be regulated by the presence of secondary structures in the promoter as it has been shown for basonuclin gene products (Tang \& Tseng, 1999).

\subsection{Regulation of the Transcription of kcna7/KCNA7}

The first report of the cloning of the human KCNA7 gene included a computational analysis of the human genomic sequence adjacent to the first exon (Kashuba et al., 2001). Regulatory regions directing skeletal muscle-specific transcription were located $\sim-1100 \mathrm{bp}$ relative to the $5^{\prime}$ end of the gene. Potential binding sites for transcription factors (TFs) were identified within this region (Kashuba et al., 2001).

Our promoter analysis encounter high modular homology between of the mouse and human kcna7/KCNA7 gene putative promoters, and very particularly the identification of three clusters of transcription factors binding sites (TFs) resulted of special interest to us. We decided to group those three sets of TFs in clusters related to muscle-specific transcription factors binding sites that we designated "myogenic", a "cell cycle related", and a cluster named "stress related " because it contained TFs for ATF6 and HIF1, both transcription factors activated in response to cell insults.

Since kcna7 and KCNA7 transcripts have been cloned from muscle tissue (skeletal and cardiac) the identification of binding sites for muscle-specific TFs gained importance. Myogenesis is characterized by the transcriptional activation of musclespecific genes encoding for contractile proteins, metabolic enzymes, ion channels, and neurotransmitter receptors (Olson, 1990; Arnold \& Braun, 2000; Brand-Sabery \& Christ, 2000). Transcription of muscle-specific genes is regulated by a set of basic helix-loop-helix muscle regulatory factors (bHLH-MRFs) that includes MYOD (Davis 
et al., 1987). Other more general factors such as the myocyte enhancer factor 2 (MEF2, Gossett et al., 1989) are essential for muscle differentiation. During skeletal muscle development MYOD (and Myf-5) are responsible for the specification and maintenance of myoblast identity. MTBF (muscle specific Mt binding site) is a $5^{\prime}$ muscle-specific enhancer that partially controls the expression of the muscle-specific intermediate filament, desmin gene, in skeletal muscle (Gao et al., 1998). This enhancer activity can be divided into myoblast-specific and myotube-specific activation domains. The myotube-specific region contains a MyoD and MEF2 sites, whereas the myoblast-specific region contains Sp1, Krox, and Mb sites. Functional synergism among the MYOD, MEF2, and Mt sites in myotubes has been demonstrated. With the exception of MYOD (that was found only in the putative mouse promoter) all other factors, MTBF, SP1 and MEF2 are present and organized in the same way in the proposed promoter sequences of both mouse and human genes pointing out to the idea that indeed the transcription of Kv1.7 channels forms constitute part of the repertoire of genes necessary during muscle development.

A very interesting finding is the localization of two binding sites for transcription factors responsive to physiological stress. In one hand, ATF6 is a member of the basic-leucine zipper family of transcription factors. It contains a transmembrane domain and is located in membranes of the endoplasmic reticulum (ER). ATF6 has been implicated in the ER stress response pathway since it can activate expression of GRP78 (a $78 \mathrm{kDa}$ glucose-regulated protein which functions as a ER molecular chaperone and a calcium-binding protein) and other genes induced by the ER stress response. ER stress appears to activate ATF6 by cleavage from the ER membrane and translocation to the nucleus. Moreover, the complex GAL4-ATF6 can be activated by ER stress. Therefore, ATF6 is a direct target of the ER stress response thus a proximal sensor of the ER stress response. And on the other hand, the cellular redox states are related to hypoxia by changes in the expression of redox-regulated genes and the generation of reactive oxygen intermediates. HIF-1 (Hypoxia induced factor-1) is part of a widespread oxygen sensing mechanism. HIF-1 DNA binding activity is only detectable in nuclear extracts of cells cultivated in a low oxygen atmosphere, being the oxygen-regulated subunit responsible for HIF activity (Wang \& Semenza, 1995). It has been shown that HIF-1 regulates the expression of AM, ET-1, iNOS and vascular endothelial growth factor in cardiac myocytes (Cormier-Regard et 
al., 1998; Jung et al., 2000; Kim et al., 2002; Kakinuma et al., 2002). Thus, the presence of a hypoxia activated TF on the proposed promoter of Kv1.7 channels could correlate with the control of the transcription of a redox sensitive protein like Kv1.7 channels, inferred from the sensitivity of mKv1.7 wt channels to the oxidizing conditions such as patch excision or addition to the bath of DTDP. Expressed in a very dynamically redox-controlled tissue like the muscle, Kv1.7 channels might serve an important role in the regulation of the muscle membrane potential especially in response to ischemia. Furthermore, the hypoxia-inducible factor (HIF)-1alpha levels are elevated in normoxic cells undergoing physiological processes involving large scale microtubule reorganization, such as embryonic development, wound healing, and tumor cell metastasis. This last piece of evidence closes somehow a circle between the cell cycle related TF cluster and the stress related one present in the suggested promoters of kcna7/KCNA7 in rodents and hominids.

Nevertheless, promoters tell relatively little about gene regulation if looked at in isolation. The actual behavior of a promoter is determined by the nature and quantities of transcription factors within the cell and cannot be determined solely from the promoter sequence. As in case of the amino acid sequence, the promoter sequence just determines, the potential kind of regulation involved in the transcription of the kcna7/KCNA7 gene, but not what is really happening in a given cell and situation. Therefore more investigation is required to clarify such interesting possibilities.

\subsection{Sequence analysis of kcna7/KCNA7}

In the case of the mouse kcna7 cDNA cloned by us the nucleotide sequence contains CGGC at the positions 123-126, which corresponds to CGC in the first murine sequence published (positions 523-525, Accession No. NM010596.1). The subsequent human and mouse "corrected" sequences, as well as the murine ESTs for Kcna7 (Accession Nos. Al322534.1 and Al324179), the working draft sequence of the mouse chromosome 7 (Accession No. AC073711), and a murine cDNA fragment corresponding to the $5^{\prime}$ end of the kcna7 gene (Accession No. AJ 409348), all coincide in the sequence CGGC in the corresponding positions. Therefore, this data 
confirm that the first kcna7 sequence published by Kalman et al. (1998) had a frame shift induced by a sequencing error. This frame shift returned an "extended" cDNA and as a consequence an "extended" amino terminal domain in the protein characterized by the Kalman group.

The analysis of the cDNA amplified by us identified an AUG start codon "in frame" with the confirmed nucleotide sequence of kcna7. It is preceded by a stop codon also "in frame", and it is located about 100 nucleotides upstream of the putative open reading frame proposed for the published human and the corrected mouse kcna7/KCNA7 sequences that encode the hKv1.7 and mKv1.7 channels (Kashuba et al., 2001; Bardien et al., 2002, respectively). Therefore, the wild type channel cloned by us contained a "premature" start codon that occurs in the $\sim 100 \mathrm{bp}$ in front of the one described for the human sequence, resulting in an extended $\mathrm{N}$ terminus. Kcna7/KCNA7 are the only mammalian Kv1 channels that are organized in 2 exons and 1 intron that differ in length between the mouse and the human (1.9 and $1.3 \mathrm{~Kb}$, respectively). The finding of a weak start codon in the mouse sequence upstream of the strong AUG codon that is conserved between mouse and human, as well as the presence of single nucleotide polymorphisms in the vicinity of the intron suggest the possibility of posttranscriptional modification that could lead to expression of different proteins from the same gene.

We amplified repeatedly the same fragment from mouse RNAs from skeletal and heart muscle using various primer pairs to prime different PCR reactions suggesting that indeed this $A \cup G$ was not the result of a polymerase error. Furthermore, the genomic sequence of the murine chromosome 7 (Accession No. AC073711, Mouse Genome Project) does contain the AUG start codon we refer to in the position $74720-74722$ (reverse complementary strand) between stop codons "in frame" that result in and ORF and translated sequence in perfect agreement with our cloning results.

One way to distinguish coding regions from non-coding regions, is to examine the frequencies of stop codons. In general a coding region has only one stop codon that terminates the region. Therefore, one strategy to detect the coding region or ORF is to look for long sequences of codons, without any stop codon. Most of the 
algorithms designed to determine ORFs use the above idea scanning the DNA sequences, looking for long ORFs in all three reading frames. Upon detecting a stop codon, the algorithm scans backward, searching for a start codon. From this point of view the AUG that we propose for the initiation of the translation of the mouse Kv1.7 channel protein represents the longest ORF within two well defined stop codons (see figure 11).

Protein translation initiation sites in eukaryotic mRNAs are reached via a scanning mechanism that predicts that translation should start at the AUG codon nearest the $5^{\prime}$ end of the mRNA (Kozak, 1999). As a result the first amino acid incorporated into the protein is a methionine. However, not all AUG (ATG in DNA sequences) codons are the beginning of a protein. The identity of the AUG flanking nucleotides seems to be crucial in determining which AUG would be translated. The most conserved and determinant position within the consensus sequences in vertebrate RNAs is a purine, usually adenine, in position -3, 3nt before the AUG codon (Kozak, 1991). Position +4 occupied by a guanine has been reported to contribute to the strength of the start site. Thus a purine in position -3 and a guanine in position +4 are used to classify the start site as a weak or strong Kozak sequence (Kozak, 1999). The potential initiation of the translation points are depicted in the mouse and the human sequences in figures 11 and 15, respectively. The AUG we propose does not have a strong Kozak sequence flanking it while the AUG reported for the human clone does. However, the absence of a good context around the first AUG codon has been predicted as the cause of "leaky scanning" which implies that some 40 S ribosomal subunits bypass the first AUG codon and initiate instead at the second (rarely the third) AUG (Kozak, 1999). Several eukaryotic mRNAs have been described in which the $5^{\prime}$ proximal AUG codon occurs in a suboptimal context and in consequence the ribosomes initiate at both AUG codons producing two proteins from one mRNA (Kozak, 1991), underscoring somehow the relevance of the $A^{-3} / G^{+4}$ context in determined natural situations. Leaky scanning could be related to different causes that include that complex secondary structures (as the one observed in the sequence of kcna7) compensate for the absence of a good context (Kozak, 1990). Furthermore, although the use of "leaky scanning" is frequently used as a means to produce two functional proteins, occasionally the presence of an upstream initiation codon has been related to regulatory reasons. Leaky scanning seems to be 
modulated by growth phase or temperature underlying interesting examples of translational regulation (Kozak, 1999). Studies performed on Kv3.3 channels revealed that the inconsistencies in the inactivation rates obtained from expression in different cell types were due to the fact that the mRNA of Kv3.3 channels had a weak Kozak consensus sequence at the first methionine start site and translation could start at a second methionine start site downstream of the $\mathrm{NH}_{2}$-terminal coding region. Mutating the nucleotide sequence surrounding the first methionine start site to one more closely resembling a Kozak consensus sequence produced currents that inactivated with a fast and voltage-dependent rate of inactivation in both $\mathrm{CHO}$ and HEK cells (Fernandez et al., 2003). These results indicate that for Kv channels this mechanism might be a "strategy" to generate more diversity of a given channel, most likely in response to a particular physiological stimulus. Other mechanism that bypass the first AUG rule in eukaryotes like "reiniciation" or "internal initiation" of the translation have been reported to occur and could be related to the differences in the start sites and current kinetics reported in the literature and observed in this work on Kv1.7 channels. More research would be needed to clarify the processes involved in the control at the transcriptional level of the kcna7/KCNA7 gene.

The electrophysiological characterization revealed that the $\mathrm{NH}_{2}$-terminal region of several $\mathrm{Kv}$ channels is important in determining the channel properties related to inactivation. This is suggesting that the first AUG codon is indeed used as the initiation of the translation of the mKv1.7 wt protein despite of a "weak" start site.

\section{4 hKv1.7}

The comparison between the published KCNA7 sequences by Kashuba and Bardien, and the human cDNA amplified by us reveals some differences that include amino acid changes $\mathrm{Y} 71 \mathrm{H}, \mathrm{A} 181 \mathrm{~V}, \mathrm{P} 189 \mathrm{R}, \mathrm{M} 418 \mathrm{~T}$ and the absence of proline in position 441. The clone obtained within this study bears the changes $\mathrm{Y} 71 \mathrm{H}+\mathrm{A} 181 \mathrm{Z}+\mathrm{P} 189 \mathrm{R}$ since it was present in $100 \%$ of the clones isolated (table 1 ). All this changes have been annotated as single nucleotide polymorphisms (SNP) in the gene card of KCNA7 in the gene bank (refer to table 1 for details and references). Accordingly, the changes S340P and F400S found in our clones are also the result of 
$\mathrm{C} / \mathrm{T}$ changes therefore we believe those nucleotide exchanges are natural SNPs not reported so far.

The occurrence of the variants mentioned above, are the result of changes of $\mathrm{C} / \mathrm{T}$ (or $\mathrm{T} / \mathrm{C}$ ) in the first or second position of the codon. In mammals RNA editing is mainly represented by deamination of cytidine-to-uridine (C-to-U) and adenosine-toinosine (that functions as guanosine) (Maas \& Rich, 2000). Accordingly, the finding of several variants, snp where $\mathrm{C} / \mathrm{T}$ or $\mathrm{T} / \mathrm{C}$ have changed in an important position in the codon, of the KCNA7 sequences is a strong suggestion of considerable RNA editing. Interestingly, the changes A181V, P189R found in all the clones isolated in the present work are encoded in the immediacy of the $1.1 \mathrm{~Kb}$ intron of KCNA7, in concordance with the close proximity of characterized editing sites to the exon/intron splice junction (Maas \& Rich, 2002). A well documented example of RNA editing is the voltage dependent potassium channel sqKv2 from squid where in a segment of 360 nucleotides up to 17 adenosines are modified in a single transcript, generating edits with altered rates of channel closure and slow inactivation (Patton et al., 1997). The extent of the editing at different sites varies depending on the activity of the editing enzymes. Thus resulting in more transcript diversity as observed by Seeburg and colleagues studying RNA editing of brain glutamate receptor channels (Seeburg et al., 1998). This line of evidence opens the possibility that KCNA7 transcripts with different functional properties might originate through RNA editing. Curiously, the transcripts of the KCNA7 gene have been found in several tissues but more importantly in heart and skeletal muscle where a cytidine deaminase termed APOBEC-2 was cloned and proven to exclusively express in those tissues but its substrate has not been identified to date (Liao et al., 1999). Further investigations have to be directed to the study of the effects that the single nucleotide polymorphisms have in the gene product of the KCNA7 gene to answer the question if there are post-transcriptional modifications associated to them. 


\subsection{Functional properties of Kv1.7 channels}

Analysis of the protein sequence encoded by the kcna7 and KCNA7 constructs confirmed the identity of voltage gated potassium channels with all the structural hallmarks of Shaker related ion channels. The absence of expression interpreted from the evidence that no current was detectable in oocytes injected with the T1 domain mutant mKv1.7- $\mathrm{TT} 1$ indicates that, the interaction of the T1 domain is indispensable for the functional expression of Kv1.7 currents either by enabling the assembly and transport to the plasma membrane of functional proteins and/or by establishing the permissive conformational states related to gating states of the channel.

Resulting from the different initiation of the translation sites the kcna7 cRNA translation rendered two proteins with different $\mathrm{N}$-terminal sequences, the one we referred as mKv1.7 wt of 489 amino acids and the second, mKv1.7-T0 with 457 amino acids. The 32 amino acids difference are circumscribed to the $\mathrm{NH}_{2}$-terminal part of the protein that is predicted to reside in the cytoplasmic side of the membrane. The cloned cDNAs encoding for the mouse and human Kv1.7 channels share 93\% homology at the amino acid level up to the area between the two start of the translation sites. Thus, mKv1.7-T0 and hKv1.7 are highly homologous.

The electrophysiological analysis of mKv1.7 wt, mKv1.7-T0 and hKv1.7 channels revealed currents with different inactivation kinetics. The steady state characteristics of these channels were almost unchanged whereas marked differences on the onset of inactivation were observed. mKv1.7 is a fast activating, fast inactivating, and potassium selective, outward rectifier channel. The main difference of mKv1.7-T0 and hKv1.7 is their very slow inactivation kinetics when compared to the mKv1.7 wild type.

The results obtained in the determination of the ionic selectivity, by measuring the reversal potential of the macroscopic tails in the presence of increasing concentrations of $\mathrm{K}^{+}$, of the wild type and mutant channels are in agreement with the fact that the primary functional effect of the deletion of the $\mathrm{N}$-terminus fragment in $\mathrm{mKv1.7-T0} \mathrm{is} \mathrm{restricted} \mathrm{to} \mathrm{channel} \mathrm{gating} \mathrm{through} \mathrm{changes} \mathrm{in} \mathrm{the} \mathrm{inactivation} \mathrm{kinetics.}$ 
Corresponding to a local action of the "distorted" inactivation domain without altering the conduction properties observed in the wild type. In general the mKv1.7-T0 characteristics are very similar to the human Kv1.7 channel. Thus, the ionic selectivity is common to all three channels investigated in agreement with the pore sequences being virtually identical.

\subsubsection{Steady state activation}

Our data demonstrate that the deletion on the $\mathrm{N}$-terminus of the mKv1.7 channel influences mildly but significantly the steady activation characteristics, thus shifting the half activation potential to more hyperpolarized voltages without altering the slope parameter of the channel. A similar effect has been reported for Kv2.1 channels (drk1) where the deletion of the most $\mathrm{NH}_{2}$-terminal 16 amino acid rendered a channel with $V_{1 / 2} \sim 5 \mathrm{mV}$ displaced to more negative potentials without changes in the slope of the activation curve (VanDongen et al., 1990). Studies by Smith-Maxwell et al. suggest that the presence of 5 positively charged amino acids in the $\mathrm{N}$-terminus from Shaker $B$ channels shifted the voltage dependence of activation to more positive potentials (Smith-Maxwell et al., 1998). The authors attributed the effect to steric interactions within the $\mathrm{S} 4$ region. Thus, the absence of 32 amino acids on the $\mathrm{N}$-terminus generated a shift of the activation threshold of mKv1.7-T0 channels to more negative potentials. Similarly the activation of mKv1.7-T0 channels resulted slowed in comparison to the wild type protein that bears the full $\mathrm{NH}_{2}$ domain. Both effects on channel gating have been reported for the rat eag channels, where a mutation in the $\mathrm{S} 4$ domain was able to reverse the effect of the $\mathrm{N}$-terminal deletion and for that reason a molecular interaction between the $\mathrm{N}$-terminus and the $\mathrm{S} 4$ segment was proposed (Terlau et al., 1997). The complete amino terminal part of mKv1.7 wt (174aa) has a net positive charge of 6.45 whereas the charge of the corresponding domain in mKv1.7-T0 (142aa) is 4.12, implying a higher potential for electrostatic interaction. We propose that, since the channel core remained essentially unaltered, as suggested for $\mathrm{r}$-eag channels interactions between the $\mathrm{N}$ terminus and the $\mathrm{S} 4$ segment could have some relevance in the effects observed by us between the wild type and mutant of mKv1.7 channels. 


\subsubsection{Inactivation}

Classically two types of inactivation are described for Kv channels. A fast or $\mathrm{N}$ type that occurs in the order of millisecond and involves the occlusion of the permeation pathway from the intracellular side; and a slow inactivation regarded as C-type inactivation due to the fact that was described for the first time in C-terminal deletion mutants of Shaker channels. The C-type inactivation can range from hundred of milliseconds to several seconds and is believed to engage the collapse of the extracellular mouth of the channel.

Exhaustive evidence from studies on the Drosophila Shaker channels, as well as other voltage activated fast inactivating ion channels, have been published on the function of the $\mathrm{N}$-terminal domain as the entity responsible for fast inactivation. The mechanism of such activity is known as the "ball and chain" model where a relatively flexible inactivation particle (ball) that is tethered (by the chain) to the channel protein gains access from the internal cavity and blocks the ion pathway from the intracellular side (Armstrong et al., 1973). Mutational analysis and crystallographic data have proven that the requirements for the inactivation particle to block the cavity of the targeted channel are not very stringent. A hydrophobic region of about ten amino acids has to precede a net positively charged stretch of about 10 amino acids (Murrel-Lagnado \& Aldrich, 1993). In the specific case of the mKv1.7 channel the "extended" N-terminus has a sequence that resembles the motives described for the inactivation peptides of Shaker channels. Deletion of specific segments of the amino terminus retarded inactivation as reported for Shaker and Kv1.4 channels (Hoshi et al., 1990; Lee et al., 1996). Likewise, the work of Furukawa on the Aplisia AKv1.1a channel shows that the deletion of the $\mathrm{N}$-terminal domain rendered a slow inactivating channel (Furukawa et al., 1995), as it is the case of the mKv1.7-T0 mutant. All this evidences are compatible with our observation of the mKv1.7-T0 mutant, and therefore the faster inactivation observed in the mouse wild type channels can be associated to a process similar to N-type inactivation.

An important consequence of the presence of 32 amino acids of the wild type channel was revealed further in the comparison of the time course of cumulative inactivation during repetitive stimulation $(0.2 \mathrm{~Hz})$. For $\mathrm{mKv} 1.7 \mathrm{wt}$ a stronger 
cumulative inactivation has been observed under the same conditions than either mKv1.7-T0 or hKv1.7. Under control conditions $\left(2.5 \mathrm{mM} \mathrm{K}^{+}\right)$, the wild type $\mathrm{mKv1.7}$ channels recovered from inactivation in a slow fashion with a time constant of about $1.2 \mathrm{sec}$, while the recovery of the human channels and Kv1.7-T0 mutants was faster $(\sim 0.8 \mathrm{sec})$. Therefore, it is expected to observe more cumulative inactivation on the mKv1.7 wt channels at this frequency of stimulation. Moreover, cumulative inactivation was modulated by extracellular potassium in all the channels studied, particularly evident in the mKv1.7 wt channels cause even small changes in the $\left[\mathrm{K}^{+}\right]_{\mathrm{o}}$ significantly speeded the cumulative inactivation rate. In agreement with our observations Baukrowitz and Yellen found that frequency-dependent cumulative inactivation of Shaker channels is sensitive to changes of $\left[\mathrm{K}^{+}\right]_{0}$ especially in the physiological range, where much more inactivation was observed at low $\left[\mathrm{K}^{+}\right]_{\mathrm{o}}$ (Baukrowitz \& Yellen, 1995). The authors conclude that such effect resulted from the interaction of $\mathrm{N}$ - and $\mathrm{C}$-type inactivation, proposing two mechanisms through which $\mathrm{N}$-type inactivation would enhance $\mathrm{C}$-type inactivation. First, $\mathrm{N}$-type inactivation might be inhibiting the outward flux of $\mathrm{K}^{+}$, which normally fills an external $\mathrm{K}^{+}$binding site that prevents C-type inactivation. Second, the presence of the inactivation particle would keep open the activation gate of the channel even after repolarization, allowing C-type inactivation to occur for a prolonged period (Baukrowitz \& Yellen, 1995). This report evidences an antagonistic effect of $\mathrm{K}^{+}$from the extracellular media over the onset of the cumulative inactivation, as we could see with Kv1.7 channels, indicating that indeed $\mathrm{N}$ - and $\mathrm{C}$ - type inactivation coexist in the channels studied. Furthermore, the graded effect observed between mKv1.7 wt and mKv1.7-T0/hKv1.7 is an indication of the extent at which of the processes occur in each of the channels characterized in this work.

Recovery from inactivation of mKv1.7 T0 as well as the human Kv1.7 channels was fast, while mKv1.7 wt recovery was speeded to the same level only in the presence of high $\mathrm{K}^{+}$. Not only the slow time constant, tau1, of mKv1.7 wt became faster in high $\mathrm{K}^{+}$but also the relative contribution of the slow component of the recovery from inactivation decreased in the presence of high $\mathrm{K}^{+}$. In parallel the fast time constant, tau2, seemed to be independent of the extracellular concentration of potassium. In accordance to these results, during the recovery from inactivation of 
Shaker $B$ channels the slow time constant tau1 resulted $\mathrm{K}^{+}$o sensitive while tau 2 was not (Gomez-Lagunas \& Armstrong, 1994). Therefore, the association between tau1 must be directly related to the N-type inactivation from which recovery is slow, while tau2 should be related to a second inactivation mechanism with slower kinetics but faster recovery. Recovery from these inactivation mechanism is facilitated by hyperpolarization. Labarca and MacKinnon proposed that high $\mathrm{K}^{+}$was able to displace the channels from an inactivated state to another from which they could be opened (Labarca \& MacKinnon, 1992). This is in agreement with the recovery from inactivation data presented in this study for the wild type mouse Kv1.7 channel. Our results demonstrate that the onset of macroscopic fast inactivation of Kv1.7 channels is independent of extracellular $\mathrm{K}^{+}$but the recovery from inactivation is $\mathrm{K}^{+}$dependent. In contrast, for T0 and human channels the slow onset of inactivation is $\mathrm{K}^{+}$ dependent but the recovery is $\mathrm{K}^{+}$independent. Furthermore, the deviation observed for the changes in the conductance induced by the decrease in the driving force of Kv1.7 channels from the theoretical values could be related to early onset of the inactivation in high $\mathrm{K}^{+}$as it will be discussed further.

In a consistent way, the recovery from inactivation of mKv1.7-T0 channels was

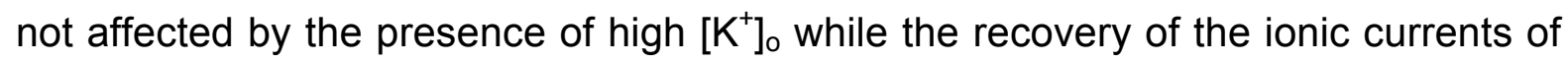
$\mathrm{mKv1.7} \mathrm{wt}$ is faster in isotonic potassium than in low external potassium (Demo \& Yellen, 1991). This supports the view that external $\mathrm{K}^{+}$destabilizes the binding of the "ball peptide" to the inner cavity of the channel (Gomez-Laguna \& Armstrong, 1994), which is additional evidence supporting the idea of mKv1.7 wt channels undergoing predominantly $\mathrm{N}$-type inactivation.

Since in the presence of high extracellular $\mathrm{K}^{+}$the recovery of $\mathrm{mKv} 1.7 \mathrm{wt}$ channels is significantly faster, the more pronounced effect of high extracellular $\mathrm{K}^{+}$on the cumulative inactivation time course of the mouse wild type channel must have been linked to the sensitivity of the fast inactivation to the extracellular increase of the extracellular potassium concentration. All this evidence is in agreement with the assumption that mKv1.7 wt channels inactivate predominantly due to a $\mathrm{N}$-type related mechanism. Hence, the mKv1.7 wt channels can be distinguished from Kv1.7-T0 and human channel forms on the basis of the different contribution that each of the rates of recovery from inactivation have. Furthermore, our results might indicate that 
inactivation and activation processes are coupled. In voltage-gated sodium channels, the two processes have been shown coupled such that inactivation derives its voltage dependence through a tight coupling to activation. Tight coupling also can be detected in delayed recovery imposed by the necessity for channels to deactivate before recovery can proceed upon repolarization (Kramer et al., 1998).

One of the hallmarks of C-type inactivation is that this process is inhibited by extracellular permeant ions such as $\mathrm{K}^{+}$and $\mathrm{Rb}^{+}$(Lopez-Barneo et al., 1993; Baukrowitz \& Yellen, 1996), as well as extracellular pore blockers like TEA. Such phenomenon is recognized as "foot in the door", where the external ion alters the rate of inactivation without altering the activation kinetics (Swenson \& Armstrong, 1981). Other types of inactivation namely P- and U-type have been described for other $\mathrm{Kv}$ channels to account for differences with the inactivation process observed in Shaker channels mutants where amino acids in the permeation pathway have proven to be determinants in the inactivation features (De Biasi, 1993). Paradoxically, we observed that the fast inactivating-channel tau inac was insensitive to $\mathrm{K}^{+}{ }_{0}$ but when exposed to TEA the inactivation became slower. Furthermore, the slow-inactivating channel was sensitive to extracellular $\left[\mathrm{K}^{+}\right]$but in an opposite way than what has been reported for classic C-type inactivation in Shaker and Kv2.1 channels (MacKinnon \& Yellen, 1990; Heginbotham \& MacKinnon, 1992).

Since, mKv1.7-T0 and hKv1.7 inactivate in a time frame of about $1 \mathrm{sec}$ we refer to this inactivation as slow inactivation that somehow must be related to C-type like process masked by the permanent fast inactivation that is apparently not evident on T0 and human channels but is rather evident in mKv1.7 wt channels. The slow inactivation observed in mKv1.7-T0 and hKv1.7 channels has mixed features that include speeding of the inactivation by high extracellular $\mathrm{K}^{+}$and insensitivity of the inactivation time constant to the block by extracellular TEA. Thus, the slow inactivation of mKv1.7-T0 and hKv1.7 channels cannot be accounted solely to the presence of C-type inactivation since our results did not evidence the most classical features associated with it. In turn mKv1.7 wt channels in the presence of TEA do inactivate slower as if the presence of the TEA could uncover the sensitivity of the Ctype inactivation that seems to be present as the fast component in the recovery from inactivation kinetics. Due to the fact that the recovery from inactivation of mouse and 
human Kv1.7 channels has two exponentials, the contribution that the slow component and the fast components, that sum up the kinetics of recovery, are used as a reporter of the extent of operation of the each of two inactivation mechanism. Accordingly, mKv1.7 wt channels have roughly the same contribution from both components during the process of recovery, but in the presence of high extracellular $\mathrm{K}^{+}$the slow component gets smaller. Conversely, the same slow time constant only contributes in $20 \%$ to the recovery of human and mKv-1.7-T0 channels that recover significantly faster, although in the presence of high $\mathrm{K}^{+}$both channels show faster onset of inactivation kinetics. According to that, the interaction of two forms of inactivation in the channels studied, hinders a clear dissection of the mechanisms. Nevertheless, it is likely that the slow component of recovery must be represented by $\mathrm{N}$-type inactivation process due to the incomplete removal of the inactivation domain. In contrast, for the molecular mechanism of the slower inactivating channels mKv1.7T0 and hKv1.7 the presence of a slow recovery component needs to be clarified.

One potential mechanism to account for the fast inactivating component of mKv1.7 wt channels would come from the mutagenesis work of Lopez-Barneo where changing amino acid T449 from Shaker to an alanine, which is the natural occurring amino acid present in the homologous position substitution in Kv1.7, was correlated with the appearance of fast C-type inactivation in Shaker B $\square$ 6-46 channels (LopezBarneo et al., 1993). However, slowing of the inactivation in the presence of high $\mathrm{K}^{+}$ was still observed in the T449A mutant contrary to our observation, though the $\mathrm{N}$ particle influence cannot be separated from the effects seen with mKv1.7 wt channels.

\subsection{Pharmacology of Kv1.7 channels}

\subsubsection{TEA sensitivity}

Wild type mKv1.7 channels showed high affinity to TEA with an $\mathrm{IC}_{50}$ of $125 \mu \mathrm{M}$ while the human Kv1.7 channels and the mKv1.7-T0 currents were blocked with an $\mathrm{IC}_{50}$ of about $2.5 \mathrm{mM}$ TEA, being about 20 fold than the $\mathrm{IC}_{50}$ for wild type channels. The sequences of $\mathrm{mKv1.7}$ wt and T0 are virtually identical, with the only difference 
being the presence of the inactivation domain in the wild type channels. Since it is known that TEA interacts with the ion channel pore the question arises: How is the deletion of the $\mathrm{N}$-terminus part can cause the observed functional effects? It is known that the "voltage dependent gating" of $\mathrm{Kv}$ channels is produced by the effects of transmembrane electric potential on the positively charged fourth domain S4. The nature of the ionic species flowing through the permeation pathway is known to influence the binding of the quaternary ammonium compound TEA (Thompson \& Begenesich, 2003). The block of mKv1.7 wt channels by TEA is voltage dependent. It has been suggested that the voltage dependence of the block by TEA of Shaker channels is related to the movement of potassium ions between the two positions of the selectivity filter that are within the membrane electric field. Thus, extracellular TEA can block Shaker B channels deprived from the inactivation domain ( $\square 6-46)$ only when the ion in the selectivity filter is away from the most external site of the ion pathway. It has been extensively documented that discrete amino acids in the extracellular mouth of the ion pore influence dramatically the sensitivity for the block of TEA as well as other blocking agents (MacKinnon \& Yellen, 1990; MacKinnon \& Miller, 1989). One such amino acid critical for the interaction of TEA and charybdotoxin with Shaker channel is T449 located in the C-terminal end of the S5S6 linker. A conservative change in T449 to tyrosine generated a 50fold increase in the sensitivity of the toxin for the channel (from 27 to $0.5 \mathrm{mM}$; MacKinnon \& Yellen, 1990). The equivalent position of T449 is occupied by a hydrophobic amino acid in mKv1.7 channels (alanine 398). Another critical amino acid for the binding of charybdotoxin to Shaker channels is in position 425, in the S5-S6 linker, where a mutation of the natural occurring $\mathrm{F}$ to an $\mathrm{H}$ generated a 8fold increase in the affinity (Perez-Cornejo et al., 1998). Kv1.7 channels naturally contain a histidine in the homologous position (mKv1.7 wt H374, hKv1.7 H341) and therefore it is expected to observe a high affinity to extracellular TEA. Moreover the amino acid residue 373 from mKv1.7 is a serine instead of threonine in the conserved positions 424 and 340 of Shaker and hKv1.7 channels, respectively.

Interestingly, the affinity of mKv1.7 wt for TEA is $\sim 125 \square \mathrm{M}$. However, an important influence of TEA on the fast inactivation could only be observed in mKv1.7 wt channels since both the human and the T0 mutants lack this fragment in the $\mathrm{NH}_{2}$ domain. Accordingly, the affinity of these two channels was 20 times lower for hKv1.7 
and mouse mutant both bearing an alanine $\left(\mathrm{IC}_{50} \sim 2.5 \mathrm{mM}\right)$ at the homologous position of the Shaker binding site for extracellular TEA. A possible explanation of this observation could be that the observed functional effects are due to conformational changes occurring in the intracellular domains that are linked to channel gating. The amino terminal deletion could either change the equilibrium between intrinsic conformational states of the channel protein, or cause a more focalized effect such as electrostatic interactions within the permeation pathway. For the inactivation ball to gain access to the transmembrane pore region through a lateral opening to gain access to the pore it must move through the T1 central cavity, suggesting that the cavity could influence or be influenced by the presence of the inactivating particle (Zhou et al., 2001). One possible interpretation is that the presence of the $\mathrm{N}$-terminus from mKv1.7 wt could favor a high affinity configuration for TEA. As shown in figure 37 there is an apparent voltage dependence of the block of mKv1.7 wt currents by TEA, but considering the model from Thomson and Begenisich it is hard to imagine how a positively charged particle like the inactivation domain of mKv1.7 channels occluding the pore from the intracellular side could induce the proposed high affinity $\mathrm{K}^{+}$ions configuration in the selectivity filter with a $\mathrm{K}^{+}$ ion occupying the innermost location in the filter. In the later case a contrary effect would be expected due to steric and/or electrostatic interaction between the inactivation particle from the cavity of the channel, the $\mathrm{K}^{+}$ions on the selectivity filter and the TEA from the pore mouth. However, the report of Thompson and Begenisich is based on the interactions of TEA with Shaker channels that contain a threonine in position 449 (Thompson \& Begenisich, 2003). The Kv1.7 channels contain an alanine instead, which is less bulky and hydrophilic than a T. Another possible explanation of the observed differences in the TEA sensitivity could be related to higher affinity of TEA to the $\mathrm{N}$-inactivated $\mathrm{mKv1.7}$ wt channels where the voltage dependence would come from unblock upon the channel opening induced by depolarization.

\subsubsection{Zinc block of Kv1.7 currents}

The interaction of zinc with Kv1.7 channels resulted in profound block of the current. This block is voltage dependent being about 2 fold more sensitive at $0 \mathrm{mV}$ than at $+40 \mathrm{mV}$ for $\mathrm{Kv1.7} \mathrm{wt}$ and 3fold different in the mKv1.7-T0 despite that the later 
is less sensitive to zinc (mKv1.7 wt $I C_{50}$ at $0 \mathrm{mV}=0.23 \pm 0.01 \mathrm{mM} ; I_{50}$ at $40 \mathrm{mV}$ $=0.43 \pm 0.002 \mathrm{mM} ; \mathrm{mKv} 1.7-\mathrm{TO} I \mathrm{IC}_{50}$ at $0 \mathrm{mV}=0.67 \pm 0.1 \mathrm{mM} ; \mathrm{IC}_{50}$ at $\left.40 \mathrm{mV}=1.9 \pm 0.6 \mathrm{mM}\right)$. The voltage dependence of the block by $\mathrm{Zn}^{2+}$ of $\mathrm{mKv1.7}$ currents suggests, as the block by TEA, that the blocker has more affinity for predominantly $\mathrm{N}$-type inactivated channel than for the slower inactivating one. The potential site for action of $\mathrm{Zn}^{2+}$ block has been suggested to depend on binding of $\mathrm{Zn}^{2+}$ to imidazole rings of histidine or to sulphur atoms in cysteines. Lower affinity has been reported to occur at the side chains of acidic amino groups over glutamate or aspartame residues (Vallee \& Auld, 1990). It has been reported that a histidine residue located on the channel turret region in position 463 in the human Kv1.5 channels is a high affinity binding site for $\mathrm{Zn}^{2+}$. Also residues E456A, D469 and D485 coordinate $\mathrm{Zn}^{2+}$ for block of hKv1.5 currents. Kv1.7 channels contain all those residues at the homologous positions (E367, H374, E379 and D396) suggesting that block should occur by a similar mechanism. However, the conformational modification associated to the $\mathrm{N}$-type inactivation of mKv1.7 wt channels must have an influence on the current sensitivity to the block as evidenced by a smaller $\mathrm{IC}_{50}$ at the potentials tested.

\subsection{3 $] \mathrm{M}$-conotoxin RIIIK}

In this study we showed that $\square \mathrm{M}$-conotoxin RIIIK blocks the mouse and human Kv1.7 channels with an affinity of about $150 \mathrm{nM}$ and $800 \mathrm{nM}$, respectively. From the other mammalian members of the Kv1 family only Kv1.2 shows affinity on the nanomolar range while all the other tested so far were insensitive to concentrations between 5-10 $\square \mathrm{M}$ of the peptide (Ferber et al., 2004). Interestingly, \-conotoxin PVIIA with pharmacological properties similar to $\square$ M-conotoxin RIIIK does not block Kv1.7 channels $\left(\mathrm{IC}_{50}>50 \square \mathrm{M}\right)$. Affinities for $\square \mathrm{M}-\mathrm{R}$ IIIK were calculated from single concentration experiments where only reduction on the peak currents from single pulses was taken into account. Further experiments with dose response curve determinations have suggested that the block is different for the different conformations of the channel (state dependence). State dependence has been previously shown for the interaction of $\square$-conotoxin PVIIA and $\square$ M-conotoxin RIIIK with Shaker channels (Terlau et al., 1999; Ferber et al., 2003) and human Kv1.2 channels (Ferber et al., 2004). Furthermore there seems to be different cooperativity 
in the block of $\square$ M-RIIIK over Kv1.7 channels (data not shown).

\subsubsection{Conkunitzin-S}

The mouse and human Kv1.7 channels are the only members of the Kv1 family of mammalian voltage activated potassium channels that are sensitive to Conkunitzin-S with an affinity in the low nanomolar range. Although the $\mathrm{IC}_{50}$ of the mouse channels to the peptide toxin are about 2.5fold higher than the human $\mathrm{IC}_{50}$. Hence, Conkunitzin-S is the only channel blocker that showed a different pattern having the highest affinity for the human channel instead of the mouse mKv1.7 wild type channel. Further studies on the kinetics parameters of the block of Conkunitzin$S$ might contribute to the clarification of the factors determining the higher affinity that the other blockers assayed had to the fast inactivating channel Kv1.7 from mouse in this work. The specificity of Conkunitzin-S indicated that this peptide might be a good tool for studying Kv1.7 channels in vitro and in vivo.

\subsubsection{Oxidation sensitivity of Kv1.7 channels}

It is known that fast inactivating Shaker $B$ channels exposed to oxidizing agents recover from inactivation faster than reduced ones. The underlying mechanism has been suggested to be a direct enzymatic oxidation of the third methionine in the $\mathrm{N}$-terminal inactivation domain of the channel that destabilizes the properties of the inactivation particle eliminating fast inactivation (Ciorba et al., 1997, 1999). Kv1.4, a mammalian channel present in cardiac tissue, is also regulated by oxidation causing slower inactivating currents which are due to patch excision or oxidation of cysteine13 on the $\mathrm{N}$-terminus, resulting in loss of fast inactivation (Ruppersberg et al., 1991). Several other examples of modulation of potassium currents by oxidation have been documented in the literature (Duprat et al., 1995; Szabo et al., 1997, Vega-Saenz de Miera \& Rudy, 1992). Accordingly, oxidation of key amino acids in the pore domain of Shaker channels (lacking the $\mathrm{N}$-inactivation domain) has been implicated in the acceleration of P-type inactivation in those channels (Chen et al., 2000). We have observed that mKv1.7 wt channel inactivates 
significantly faster upon patch excision and in presence of the oxidizing agent dithiodipyridine while washing lead to reestablishment of slower inactivation kinetics. The oxidative modulation of the Kv1.7 current does not only affect the inactivation time constant but causes a decrease in the current amplitude. We could not observe a faster inactivation of mKv1.7-T0 currents upon patch excision nor speeding of the current decay in the presence of DTDP in the human Kv1.7 channels. That indicates the relevance of the $\mathrm{N}$-terminal domain in the expression of the phenomenon. The analysis of the $\mathrm{N}$-terminus of mKv1.7 wt channels revealed the presence of several features that strongly suggest its involvement as an inactivation particle with characteristics closely resembling the ball domain from Shaker. More over, the investigation of the structural aspects of the inactivation domain of Kv1.7 revealed the presence of a particular pattern of an iron-sulfur cluster (FeS) coordinated by a

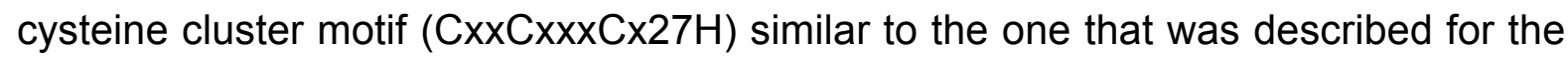
nqo3 subunit of the proton translocating NADH-Quinone oxidoreductase (NDH-1) from Thermus thermophilus HB-8 (Nakamura-Ogiso et al., 2002). Iron-sulfur proteins are ubiquitous in nature containing sites with one to eight iron atoms, sometimes repeated within the same protein (Beinert et al., 1997). Iron-sulfur clusters have a remarkable facility for conversion and interconversion in the free and protein bound conditions supporting the concept that they are modular structures that can undergo ligand exchange reactions and oxidative degradation, both of high biological significance. Enzymes bearing FeS clusters like aconitase are modulated by changes in the $\mathrm{pH}$ or thiol attack (Kennedy et al., 1984). From the structure/function relation point of view it is particularly interesting the finding of an iron-sulfur coordination motif in the N-terminus of mKv1.7 wt channels. Such motives are known to bind cysteine ligands from different subunits where iron sulfur clusters effect dimer formation as in the Fe protein nitrogenase (Howard \& Rees, 1996). Even more exciting is the fact that iron-sulfur clusters serve as sensors of iron, dioxigen, superoxide ion $\left(\mathrm{O}_{2}{ }^{-}\right)$and possibly nitric oxide (Rouault et al., 1992; Hentze \& Kühn, 1996; Beinert \& Kiley, 1996; Gaudu \& Weiss, 1996; Hidalgo et al., 1995). There have been at least two modes of sensing described so far (for a review of the mechanisms see Beinert et al., 1997). Therefore, supported on the results that neither mKv1.7-T0 nor the human Kv1.7 showed sensitivity to redox modulation, it seems plausible that the oxidation sensitive region (or an important component of it) of the Kv1.7 channel must lie in the amino terminal part of the protein. A similar arrangement $\mathrm{HX}_{5} \mathrm{CX}_{20} \mathrm{CC}$ has been 
suggested to coordinate $\mathrm{Zn}^{2+}$ in the $\mathrm{N}$-terminal, cytoplasmic tetramerization domain (T1) of Shab, Shaw and Shal subfamily members, but is not found in Shaker subfamily members (Bixbi et al., 1999). The presence of a motif used by many other proteins for sensing the redox state of the cell might indicate that at least for mKv1.7 wt channels the formation of an iron-sulfur cluster in the inactivation domain or between the $\mathrm{N}$-terminus of different subunits could function as the redox sensor that triggers different responses including closure in response to determined stimulus. Furthermore, such biochemical structures are known to drive rearrangement of the protein structure by pure electrochemical coordination as a mean for fast and sensitive mechanism of functioning.

\subsection{Comparison of the results obtained in this work to the available data}

Table 12 compiles the main characteristics of mKv1.7 channels from the reports of Kalman et al. (1998), Bardien et al. (2002), and this work. It can be seen that in the report from Kalman et al. the murine Kv1.7 channel currents evoked in response to an IV stimulation protocol exhibited fast activating and very fast inactivating kinetics. In contrast the mKv1.7 channels from the Bardien et al. study were very slow inactivating. From our results we can affirm that the reason for such a difference lays in the different $\mathrm{N}$-terminal sequences that each of the channels had, the Kalman sequence has 76 amino terminal amino acids more than the Bardien clone. The currents from Kalman's work were recorded in the whole cell configuration of the patch clamp technique, therefore no oxidation effect upon patch excision can account for the speed of the inactivation. Thus, the 76 extra $\mathrm{N}$-terminal amino acids might be acting as an inactivation peptide and produce $\mathrm{N}$-type inactivation and fast kinetics as reported by Kalman et al.. Comparison of the pharmacology reveals several inconsistencies between the two reports of the same group.

When compared the data from the reports of Kalman et al. with our results on mKv1.7 channels it can be observed that our mKv1.7 wt channels activate at more depolarized potentials and inactivates rather slower than the mKv1.7 channels from Kalman. Additionally, the mKv1.7 wt channels characterized in this work were 
Table 12.

Comparison of the characteristics of mKv1.7 channels

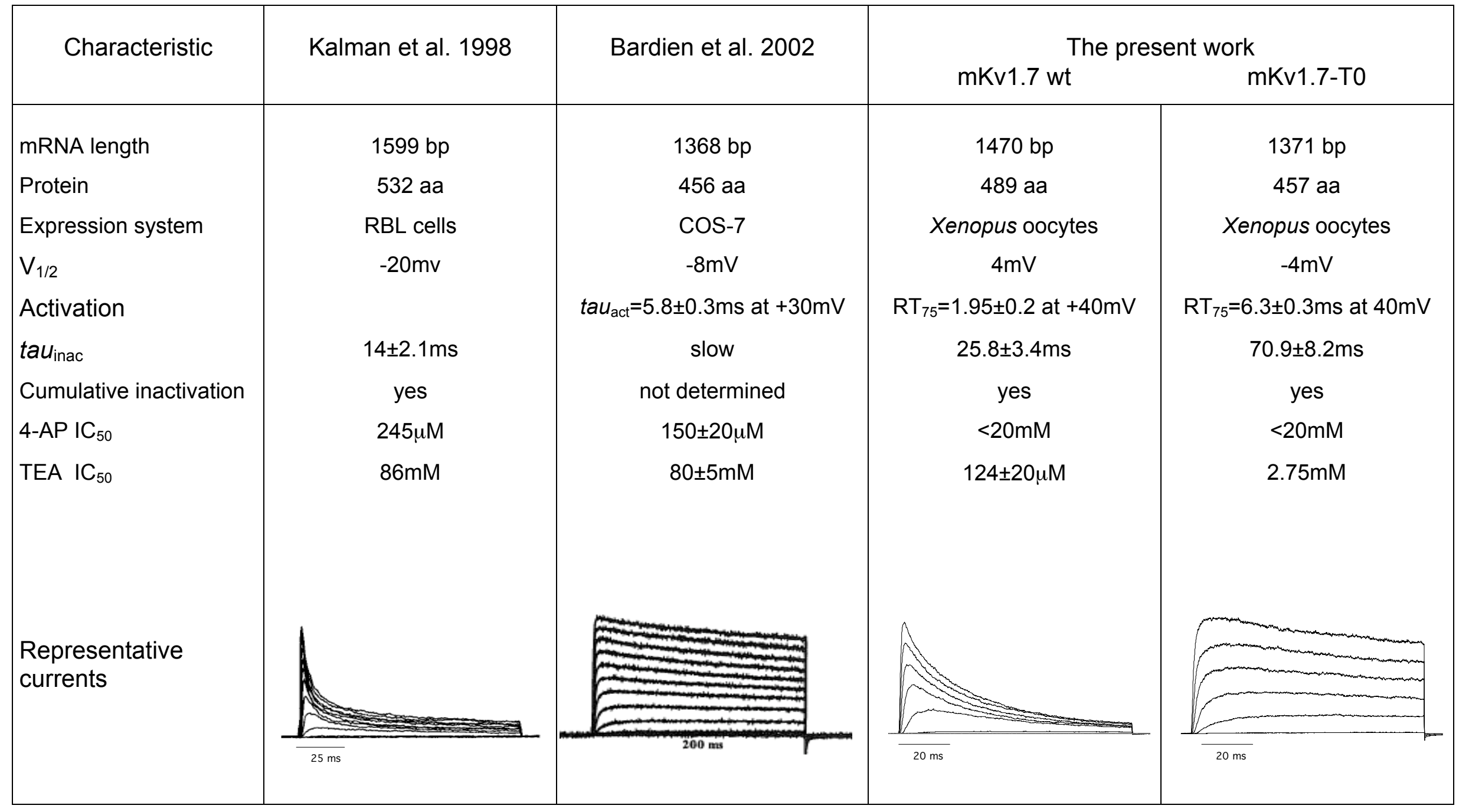


sensitive to TEA in the micromolar range and insensitive to 4-AP in contrast to the mentioned report. However, it is consistent with our results the presence of cumulative inactivation observed in the study of Kalman. Evidently, there must be an explanation to the differences observed between both studies. The core channel sequence remained almost identical excluding the divergent $\mathrm{N}$-termini. The different kinetics could be accounted for the different expression systems used, we used the Xenopus laevis expression system and they expressed the channels in mammalian cells. However, the authors state in their report that they had similar result with RBL cells and Xenopus oocytes, unfortunately this data was not presented in the article.

Due to the marked difference observed between the start codons of our mouse clone and the human clone, we generated the mKv1.7-T0 mutant that has virtually identical sequence to the clone of Bardien. When compared to the mouse Kv1.7 currents studied by Bardien et al. the values reported by them, although different to what we observed, were closer to the description of the kinetics of mKv1.7-T0 (Table 12), nevertheless the pharmacology remained puzzling since the Bardien mKv1.7 channels were blocked by 4-AP and insensitive to TEA whereas $\mathrm{mKv1.7-T0}$ in our hands is insensitive to 4-AP $\left(\mathrm{IC}_{50}>68 \mathrm{mM}\right)$ and sensitive to TEA.

As remarked previously the striking dissimilarities between the reports on mKv1.7 and our results suggest different issues. We proposed previously that the differences observed between the start of the transcription sites of mouse and human might indicate some kind of regulation at the transcriptional level. If this turns out to be true it is possible that the differences in the kinetics observed between each mKv1.7 channel characterized so far are due to different "regulation states" depending on the cell type and the conditions in which the cloning and the characterization where performed. Still the differences in the affinities to TEA and 4AP remain a mystery since both quaternary compounds have been used and are currently used extensive and very reliably in the characterization of almost every potassium channel under investigation in the world. Finally, it is relevant to point out that since the date of publication of both papers no other report has appeared to confirm or justify the marked differences reported by different authors about the mouse Kv1.7, while this work represents the first functional characterization of the human Kv1.7 channels. 
1. Kv1.7 channels are voltage sensitive and highly selective for potassium. They activate between -4 and $+4 \mathrm{mV}$ and have a voltage dependence of $15 \mathrm{mV}$ per e fold change in open probability.

2. mKv1.7 wt channels inactivate faster than mKv1.7-T0 and human channels. And this faster inactivation kinetics are positively correlated to the presence of 32 amino acids in the $\mathrm{N}$-terminus that have the characteristics of the inactivation peptides described for $\mathrm{Kv}$ channels. Thus, the fast component of the inactivation of $\mathrm{mKv1.7} \mathrm{wt}$ is predominantly due to a $\mathrm{N}$-type inactivation process.

3. Kv1.7 channels undergo cumulative inactivation.

4. Kv1.7 channels are blocked by TEA and $\mathrm{Zn}^{2+}$ in the low millimolar range and by conotoxins $\square \mathrm{M}-\mathrm{R} I I \mathrm{~K}$ and Conkunitzin-S with affinities in the low nanomolar range.

5. Fast inactivating mKv1.7 channels are sensitive to redox modulation by experiencing significant decrease in the current amplitude and a substantial enhancement of the inactivation kinetics upon oxidative conditions.

\section{Perspectives}

The activity of voltage-gated potassium (Kv) channels is crucial in the control of the membrane potential, which subsequently regulates cytoplasmic free calcium concentration, [Ca $\left.2^{+}\right]_{\text {cyt }}$ (Salvaterra \& Goldman, 1993). For instance, in cells of the pulmonary artery smooth muscle (PASMCs) acute hypoxia is known to inhibit $\mathrm{Kv}$ channel function, inducing membrane depolarization and the rise in $\left[\mathrm{Ca}^{2+}\right]_{\mathrm{cyt}}$ that triggers vasoconstriction (Gelband \& Gelband, 1997). Prolonged hypoxia is directly involved in the inhibition of the expression of $\mathrm{Kv}$ channels and the reduction of $\mathrm{Kv}$ channel currents in pulmonary arterial smooth muscle (PASMCs). Acute hypoxia decreases $I_{K^{+}}$by inhibiting $\mathrm{Kv}$ channel activity in PASMCs, with the resultant membrane depolarization attenuating Kv channel expression (Levitan et al., 1995). Therefore, $\mathrm{Kv}$ channel activity is regulated directly on the channel protein by local modulation and on the transcriptional level. Kv1.7 transcripts have been thoroughly found in muscle tissue and pulmonary rat arteries (Kalman et al., 1998; Kashuba et 
al., 2001; Davies \& Kozlowski, 2001). Our results offer good evidences to believe that Kv1.7 channels might be relevant in the muscle physiology since it seems to be regulated by stress responsive TFs and it's kinetics are directly influenced by the redox state. Finally, Kv1.7 channels seem to be the natural targets of at least two highly specific conotoxins, implying that these pharmacological tools can be used to define the function of Kv1.7 channels on health and diseased settings. 


\section{SUMMARY}

Ion channels are specialized membrane proteins that let ions pass through at a high rate when they are in the open conformation. Voltage gated $\mathrm{K}^{+}$channels are a heterogeneous group of proteins known to modulate synaptic transmission and secretion from endocrine cells, such as insulin from pancreatic islet cells. As an example, $\mathrm{K}^{+}$currents $\left(\mathrm{I}_{\mathrm{K}^{+}}\right)$play critical roles in determining cardiac electrical activities. Besides stabilizing resting potential, $\mathrm{I}_{K_{+}}$in cardiac cells also plays an important role in modulating cellular excitability and regulating membrane repolarization. Moreover, dysfunction of $\mathrm{Kv}$ channels is associated to multiple pathophysiological conditions. Hence, $\mathrm{K}^{+}$channels have important functions at the cellular level and much interest has been focused on the study of ion channels.

In the present study the murine and human orthologs of the Kv1.7 channels (kcna7 and KCNA7 genes, respectively) have been cloned using PCR and PCR related techniques. Transcripts of the kcna7/KCNA7 genes are reported to be present in several tissues including strong expression in the heart and skeletal muscle (Kalman et al., 1998; Kashuba et al., 2001). For the mouse gene two potential initiation of the translation sites have been identified which might be of functional importance. Therefore two constructs were generated one with the longest resulting open reading frame named mKv1.7 wild type (mKv1.7 wt) and a second clone called mKv1.7-T0 with a product 32 amino acid shorter as a result of the initiation at the second AUG. The human sequence only presented one initiation site corresponding to the second AUG identified in the mouse resulting in a construct, hKv1.7, homologous to Kv1.7-T0. The analysis of the sequence of the kcna7/KCNA7 genes suggests several interesting aspects that include highly controlled transcription and possibly postrancriptional modifications. The data gathered in this work differed in various aspects to what was previously reported about the mouse Kv1.7 channels. The main differences were due to single nucleotide changes in the DNA sequences that led to protein products with different amino termini.

To date only little is known about the biophysical and pharmacological characteristics of the murine Kv1.7 channels and some data are even controversial. 
In addition there is no biophysical or pharmacological information available on the human ortholog channel. In order to identify the source of the differences observed between our results and the published data, a mouse Kv1.7 construct equivalent to the clone reported by Bardien et al. in 2002 (mKv1.7-T0) was characterized in parallel to mKv1.7 wt channels. Our results include the first biophysical and pharmacological characterization of the human Kv1.7 channel.

The electrophysiological characterization of the mouse and human Kv1.7 channels was performed using two electrodes voltage clamp and patch clamp techniques by using the Xenopus laevis oocyte heterologous expression system. $\mathrm{Kv} 1.7$ channels activate between -4 and $+4 \mathrm{mV}$ with relatively fast activation kinetics. Interestingly, the wild type mouse channels inactivate about 3fold faster than mKv1.7-T0 and expectedly the hKv1.7. The faster inactivation kinetics of mKv1.7 wt channels is positively correlated to the presence of 32 amino acids in the N-terminus that are absent in the mKv1.7-T0 and hKv1.7. The N-terminal 32aa from mKv1.7 wt channels include a hydrophobic and a positively charged region as reported for the inactivation peptides described for other Kv channels including Shaker. Thus the fast component of the inactivation of $\mathrm{mKv1.7} \mathrm{wt}$ is predominantly due to $\mathrm{N}$-type inactivation that can be modulated by increasing concentrations of $\left[\mathrm{K}^{+}\right]_{0}$. Kv1.7 channels undergo cumulative inactivation that is also sensitive to $\left[\mathrm{K}^{+}\right]_{0}$.

The pharmacological profile of the mouse and human Kv1.7 channels includes sensitivity to extracellularly applied TEA and $\mathrm{Zn}^{2+}$ in the low millimolar range. Kv1.7 channels are blocked by the conotoxins $\square \mathrm{M}-\mathrm{RIIIK}$ and Conkunitzin-S with affinities in the low nanomolar range, identifying Kv1.7 channels as a target for these new families of conus peptides. Furthermore, mKv17 wt was sensitive to redox modulation with a concomitant decrease in the current amplitude and a substantial enhancement of the inactivation rate under oxidative conditions.

The findings of the present study demonstrate the functional properties of the mammalian voltage activated $\mathrm{K}^{+}$channels $\mathrm{mKv} 1.7$ and $\mathrm{hKv} 1.7$ amplified from skeletal and cardiac muscle from mouse and human by means of reverse transcription and polymerase chain reaction. The expression profile, putative promoter analysis and redox modulation of the kinetics of Kv1.7 channels suggested 
that this channels might be a potential molecular component of hypoxia-induced membrane depolarization responses in muscle cells. Therefore, many interesting open questions on the physiological role of Kv1.7 channels await further investigation. 


\section{REFERENCES}

Aldrich, R.W. 2001. Fifty years of inactivation. Nature 411:643-644.

Antz, C., Geyer, M., Fakler, B., Schott, M.K., Guy, H.R., Frank, R., Ruppersberg, J.P. and Kalbitzer, H.R. 1997. NMR structure of inactivation gates from mammalian voltage-dependent potassium channels. Nature 385:272-275.

Armstrong, C.M. 1971. Interaction of tetraethylammonium ion derivatives with the potassium channels of giant axons. J. Gen. Physiol. 58:413-437.

Armstrong, C.M., Bezanilla, F. and Rojas, E. 1973. Destruction of sodium conductance inactivation in squid axons perfused with pronase. J. Gen. Physiol. 62:375-391.

Armstrong, C.M. 1975. Ionic pores, gates, and gating currents. Q. Rev. Biophys. 7:179-210.

Armstrong, C.M. and Taylor, S.R. 1980. Interaction of barium ions with potassium channels in squid giant axons. Biophys. J. 30:473-488.

Armstrong, C.M. and Hille, B. 1998. Voltage-gated ion channels and electrical excitability. Neuron 20:371-380.

Armstrong, C.M. and Loboda, A. 2001. A model for 4-aminopyridine action on $\mathrm{K}^{+}$ channels: similarities to tetraethylammonium ion action. Biophys. J. 81:895-904.

Arnold, H.H. and Braun, T. 2000. Genetics of muscle determination and development. Curr. Top. Dev. Biol. 48:129-164.

Bajic, V.B., Chong , A., Seah, S.H. and Brusic, V. 2002. Intelligent System for Vertebrate Promotor Recognition. IEEE Intelligent Systems 17:64-70.

Bajic, V.B., Seah S.H., Chong A., Zhang G., Koh J.L. and Brusic V. 2002. Dragon Promoter Finder: recognition of vertebrate RNA polymerase II promoters. Bioinformatics 18:198-199.

Bajic V.B. and Brusic V. 2003. Computational detection of vertebrate RNA polymerase II promoters. Methods Enzymol. 370:237-250.

Bajic, V.B., Seah, S.H., Chong, A. , Krishnan, S.P.T., Koh, J.L.Y. and Brusic, V. 2003. Computer model for recognition of functional transcription start sites in polymerase II promoters of vertebrates. Journal of Molecular Graphics \& Modeling 21:323-332.

Baukrowitz, T. and Yellen, G. 1995. Modulation of $\mathrm{K}^{+}$current by frequency and external $\left[\mathrm{K}^{+}\right]$: a tale of two inactivation mechanism. Neuron 15:951-960. 
Baukrowitz, T. and Yellen, G. 1996. Use-dependent blockers and exit rate of the last ion from the multi-ion pore of a $\mathrm{K}^{+}$channel. Science 271:653-656.

Bardien, S., Wulff, H., Arieff, Z., Brink, P., Chandy, K.G. and Corfield, V. 2002. Characterisation of the human voltage-gated potassium channel gene, KCNA7, a candidate gene for inherited cardiac disorders, and its exclusion as cause of progressive familial heart block I (PFHBI). Eur. J. Hum. Genet. 10:36-43.

Beinert, H. and Kiley, P. 1996. Redox control of gene expression involving iron-sulfur proteins. Change of oxidation-state or assembly/disassembly of Fe-S clusters? FEBS Lett. 382:218-221.

Beinert, H., Holm, R.H and Munck, E. 1997. Iron-sulfur clusters: nature's modular, multipurpose structures. Science 277:653-659.

Bezanilla, F., Perozo, E., Papazian, D.M. and Stefani, E. 1991. Molecular basis of gating charge immobilization in Shaker potassium channels. Science 254:679-683.

Bezanilla, F. 2000. The voltage sensor in voltage-dependent ion channels Physiol. Rev. 80:555-592. Erratum in: Physiol Rev. 80.

Bixby, K.A., Nanao, M.H., Shen, N.V., Kreusch, A., Bellamy, H., Pfaffinger, P.J. and Choe, S. 1999. $\mathrm{Zn}^{2+}$-binding and molecular determinants of tetramerization in voltage-gated $\mathrm{K}^{+}$channels. Nat. Struct. Biol. 6:38-43.

Blaustein, R.O. and Miller, C. 2004. Ion channels: shake, rattle or roll? Nature 2004 427:499-500.

Brand-Saberi, B. and Christ, B. 2000. Evolution and development of distinct cell lineages derived from somites. Curr. Top. Dev. Biol. 48:1-42.

Brodsky, L.I., Vasiliev, A.V., Kalaidzidis, Ya, L., Osipov, Yu, S., Tatuzov, R.L. and Feranchuk, S.I. 1992. GeneBee: the program package for biopolymer structure analysis. Dimacs 8:127-139.

Brodsky, L.I., Ivanov, V.V., Kalaidzidis, Ya., L., Leontovich, A.M., Nikolaev, V.K., Feranchuk, S.I. and Drachev, V.A. 1995. GeneBee-NET: Internet-based server for analyzing biopolymers structure. Biochemistry 60:923-928.

Bretschneider, F., Wrisch, A., Lehmann-Horn, F. and Grissmer, S. 1999. External tetraethylammonium as a molecular caliper for sensing the shape of the outer vestibule of potassium channels. Biophys. J. 76:2351-2360.

Bunn, H.F. and Poyton, R.O. 1996. Oxygen sensing and molecular adaptation to hypoxia. Physiol. Rev. 76:839-885.

Carmeliet, E. 1999. Cardiac ionic currents and acute ischemia: from channels to arrhythmias. Physiol Rev. 79:917-1017.

Cha, A. and Bezanilla, F. 1997. Characterizing voltage-dependent conformational changes in the Shaker $\mathrm{K}^{+}$channel with fluorescence. Neuron 19:1127-1140. 
Chandy, K.G. and Gutman, G.A. 1993. Nomenclature for mammalian potassium channel genes. Trends Pharmacol. Sci. 14:434.

Chen, J., Avdonin, V., Ciorba, M.A., Heinemann, S.H. and Hoshi, T. 2000. Acceleration of P/C-type inactivation in voltage-gated $\mathrm{K}(+)$ channels by methionine oxidation. Biophys. J. 78:174-187.

Choe, S., Kreusch, A. and Pfaffinger, P.J. 1999. Towards the three-dimensional structure of voltage-gated potassium channels. Trends Biochem. Sci. 24:345-349.

Choi, K.L., Aldrich, R.W. and Yellen, G. 1991. Tetraethylammonium blockade distinguishes two inactivation mechanisms in voltage-activated $\mathrm{K}^{+}$channels. Proc. Natl. Acad. Sci. USA 88:5092-5095.

Choi, K.L., Mossman, C., Aube, J. and Yellen, G. 1993. The internal quaternary ammonium receptor site of Shaker potassium channels. Neuron 10:533-541.

Ciorba, M.A., Heinemann, S.H., Weissbach, H., Brot, N. and Hoshi T. 1997. Modulation of potassium channel function by methionine oxidation and reduction. Proc. Natl. Acad. Sci. USA 94:9932-9937.

Ciorba, M.A., Heinemann, S.H., Weissbach, H., Brot, N. and Hoshi T. 1999. Regulation of voltage-dependent $\mathrm{K}+$ channels by methionine oxidation: effect of nitric oxide and vitamin C. FEBS Lett. 442:48-52.

CLONTECH Marathon cDNA Amplification Kit User Manual, CLONTECH PT1115-1 (PR8Y870) Catalog \# : K1802-1

Conforti, L., Bodi, I., Nisbet, J.W. and Millhorn, D.E. 2000. O2-sensitive $\mathrm{K}^{+}$channels: role of the Kv1.2 $\square$-subunit in mediating the hypoxic response. J. Physiol. 524:783-793.

Cormier-Regard, S., Nguyen, S.V. and Claycomb, W.C. 1998. Adrenomedullin gene expression is developmentally regulated and induced by hypoxia in rat ventricular cardiac myocytes. J. Biol. Chem. 273:17787-17792.

Cortes, D.M., Cuello, L.G. and Perozo, E. 2001. Molecular architecture of full-length KcsA: role of cytoplasmic domains in ion permeation and activation gating. J. Gen. Physiol. 117:165-180.

Cushman, S.J., Nanao, M.H., Jahng, A.W., DeRubeis, D., Choe, S. and Pfaffinger, P.J. 2000. Voltage dependent activation of potassium channels is coupled to T1 domain structure. Nature Struct. Biol. 7:403-407.

Davis, R.L., Weintraub, H. and Lassar, A.B. 1987. Expression of a single transfected cDNA converts fibroblasts to myoblasts. Cell 51:987-1000.

Davies, A.R. and Kozlowski, R.Z. $2001 \mathrm{Kv}$ channel subunit expression in rat pulmonary arteries. Lung 179:147-161. 
De Biasi, M., Hartmann, H.A., Drewe, J.A., Taglialatela, M., Brown, A.M. and Kirsch, G.E. 1993. Inactivation determined by a single site in $\mathrm{K}^{+}$. Pflügers Arch. 422:354-363.

Demo, S.D. and Yellen, G. 1991. The inactivation gate of the Shaker $\mathrm{K}^{+}$channel behaves like an open-channel blocker. Neuron 7:743-753.

Doyle, D. A., Morais Cabral, J., Pfuetzner, R.A., Kuo, A., Gulbis, J.M., Cohen, S.L., Chait, B.T. and MacKinnon, R. 1998. The structure of the potassium channel: molecular basis of $\mathrm{K}^{+}$conduction and selectivity. Science 280:69-77.

Duprat, F., Lesage, F., Guillemare, E., Fink, M., Hugnot, J.P., Bigay, J., Lazdunski, M., Romey, G. and Barhanin, J. 1995. Heterologous multimeric assembly is essential for K+ channel activity of neuronal and cardiac G-protein-activated inward rectifiers. Biochem. Biophys. Res. Commun. 212:657-663.

Ferber, M., Sporning, A., Jeserich, G., De La Cruz, R., Watkins, M., Olivera, B.M. and Terlau, H. 2003. A novel conus peptide ligand for $\mathrm{K}+$ channels. J. Biol. Chem. 278:2177-2183.

Ferber, M., Al-Sabi, A., Stocker, M., Olivera, B. and Terlau, H. 2004. Identification of a mammalian target of $\square \mathrm{M}$-contotoxin RIIIK. Toxicon (in press).

Fernandez, F.R., Morales, E., Rashid, A.J., Dunn, R.J. and Turner, R.W. 2003. Inactivation of Kv3.3 potassium channels in heterologous expression systems. J. Biol. Chem. 278:40890-40898.

Frech, G.C., VanDongen, A.M., Schuster, G., Brown, A.M. and Joho, R.H. 1989. A novel potassium channel with delayed rectifier properties isolated from rat brain by expression cloning. Nature 340:642-645.

Furukawa, Y. 1995. Accumulation of inactivation in a cloned transient $\mathrm{K}^{+}$channel (AKv1.1a) of Aplysia. J. Neurophysiol. 74:1248-1257.

Furukawa, Y., Kim, H.N. and Kubo, T. 1995. Up- and down-modulation of a cloned Aplysia $\mathrm{K}^{+}$channel (AKv1.1a) by the activators of protein kinase C. Zoolog Sci. 12:35-44.

Gao, J., Li, Z. and Paulin, D. 1998. A novel site, Mt, in the human desmin enhancer is necessary for maximal expression in skeletal muscle. J. Biol. Chem. 273:6402-6409.

Gaudu, P. and Weiss, B. 1996. SoxR, a [2Fe-2S] transcription factor, is active only in its oxidized form. Proc. Natl. Acad. Sci. USA 93:10094-10098.

Gelband, C.H. and Gelband, H. 1997. Ca $2^{+}$release from intracellular stores is an initial step in hypoxic pulmonary vasoconstriction of rat pulmonary artery resistance vessels. Circulation 96:3647-3654.

Gerard, G.F. 1995. 102 Inhibition of SUPERSCRIPT Tм II Reverse Transcriptase by Common Laboratory Chemicals FOCUS $® 16,102$.

Gomez-Lagunas, F. and Armstrong, C.M. 1994. The relation between ion 
permeation and recovery from inactivation of ShakerB $\mathrm{K}^{+}$channels. Biophys. J. 67:1806-1815.

Gomez-Lagunas, F. and Armstrong, C. M. 1995. Inactivation in ShakerB K ${ }^{+}$ channels: a test for the number of inactivating particles on each channel. Biophys. J. 68:89-95.

Gosset, W.S. 1958. "The Probable Error of a Mean." "Student's" Collected Papers. Ed. E.S. Pearson and John Wishart. Cambridge: University Press.

Gossett, L.A., Kelvin, D.J., Sternberg, E.A. and Olson, E.N. 1989. A new myocytespecific enhancer-binding factor that recognizes a conserved element associated with multiple muscle-specific genes. Mol. Cell. Biol. 9:5022-5033.

Gurdon, J.B., Lane, C.D., Woodland, H.R. and Marbaix, G. 1971. Use of frog eggs and oocytes for the study of messenger RNA and its translation in living cells. Nature 233:177-182.

Hamill, O.P., Marty, A., Neher, E., Sakmann, B. and Sigworth, F.J. 1981. Improved patch-clamp techniques for high-resolution current recording from cells and cell-free membrane patches. Pflügers Arch. 39:85-100.

Hartmann, E., Wiedmann, M. and Rapoport, T.A. 1989. A membrane component of the endoplasmic reticulum that may be essential for protein translocation. EMBO J. 8:2225-2229.

Heginbotham, L. and MacKinnon, R. 1992. The aromatic binding site for tetraethylammonium ion on potassium channels. Neuron 8:483-91.

Heinemann, S. H. 1995. Guide to data acquisition and analysis. In: Single-Channel Recording. B. Sakmann and E. Neher. New York and London, Plenum Press. Chapt. 3:53-91.

Heinemann, S.H., Rettig, J., Wunder, F. and Pongs, O. 1995. Molecular and functional characterization of a rat brain $\mathrm{Kv}$ beta 3 potassium channel subunit. FEBS Lett. 377:383-389.

Heinemann, S.H., Rettig, J., Graack, H.R. and Pongs, O. 1996. Functional characterization of Kv channel beta-subunits from rat brain. J. Physiol. 493:625-633.

Hentze, M.W. and Kühn, L.C. 1996. Molecular control of vertebrate iron metabolism: mRNA-based regulatory circuits operated by iron, nitric oxide, and oxidative stress. Proc. Natl. Acad. Sci. USA 93:8175-8182.

Hidalgo, E., Bollinger, J.M. Jr., Bradley, T.M., Walsh, C.T. and Demple, B. 1995. Binuclear [2Fe-2S] clusters in the Escherichia coli SoxR protein and role of the metal centers in transcription. J. Biol. Chem. 270:20908-20914.

Hille, B., Armstrong, C. M. and MacKinnon, R. 1999. Ion channels: from idea to reality. Nature Med . 5:1105-1109. 
Hille, B. 2001. Ionic channels of excitable membranes. Sinauer Associates, Inc. Sunderland, Massachusetts, Third Edition.

Hoshi, T., Zagotta, W.N. and Aldrich, R.W. 1990. Biophysical and molecular mechanisms of Shaker potassium channel inactivation. Science 250:533-538.

Hoshi, T., Zagotta, W.N. and Aldrich, R.W. 1991. Two types of inactivation in Shaker $\mathrm{K}^{+}$channels: effects of alterations in the carboxy-terminal region. Neuron 7:547-556.

Howard, J.B. and Rees, D.C. 1996. Structural Basis of Biological Nitrogen Fixation. Chem. Rev. 96:2965-2982.

Immke, D. and Korn, S.J. 2001. Ion-Ion interactions at the selectivity filter. Evidence from $\mathrm{K}^{+}$-dependent modulation of tetraethylammonium efficacy in Kv2.1 potassium channels. J. Gen. Physiol. 15:509-518.

Isacoff, E.Y., Jan, Y.N. and Jan, L.Y. 1990. Evidence for the formation of heteromultimeric potassium channels in Xenopus oocytes. Nature 345:531-534.

Jan, L.Y. and Jan, Y.N. 1997. Cloned potassium channels from eukaryotes and prokaryotes. Ann. Rev. Neurosci. 20:91-123.

Jiang, X., Bett, G.C., Li, X., Bondarenko, V.E. and Rasmusson, R.L. 2003. C-Type inactivation involves a significant decrease in the intracellular aqueous pore volume of Kv1.4 K+ channels expressed in Xenopus oocytes. J. Physiol. 549(Pt3):683-695.

Jerng, H.H., Shahidullah, M. and Covarrubias, M. 1999. Inactivation gating of Kv4 potassium channels: molecular interactions involving the inner vestibule of the pore. $J$ Gen. Physiol. 113:641-660.

Jung, F., Palmer, L.A., Zhou, N. and Johns, R.A. 2000. Hypoxic regulation of inducible nitric oxide synthase via hypoxia inducible factor-1 in cardiac myocytes. Circ. Res. 86:319-25.

Jung, F., Haendeler, J., Hoffmann, J., Reissner, A., Dernbach, E., Zeiher, A.M. and Dimmeler, S. 2002. Hypoxic induction of the hypoxia-inducible factor is mediated via the adaptor protein Shc in endothelial cells. Circ. Res. 91:38-45.

Kakinuma, Y., Miyauchi, T., Suzuki, T., Yuki, K., Murakoshi, N., Goto, K. and Yamaguchi I. 2002. Enhancement of glycolysis in cardiomyocytes elevates endothelin-1 expression through the transcriptional factor hypoxia-inducible factor-1 alpha. Clin. Sci. (Lond). 103 Suppl 48:210S-214S.

Kalman, K., Nguyen, A., Tseng-Crank, J., Dukes, I.D., Chandy, G., Hustad, C.M., Copeland, N.G., Jenkins, N.A., Mohrenweiser, H., Brandriff, B., Cahalan, M., Gutman, G.A. and Chandy, K.G. 1998. Genomic organization, chromosomal localization, tissue distribution, and biophysical characterization of a novel mammalian Shaker-related voltage-gated potassium channel, Kv1.7. J. Biol. Chem. 273:5851-5857. 
Kamb, A., Tseng-Crank, J. and Tanouye, M.A. 1986. Multiple products of the Drosophila Shaker gene may contribute to potassium channel diversity. Neuron 1:421-430.

Kamb, A, Iverson, L.E. and Tanouye, M.A. 1987. Molecular characterization of Shaker, a Drosophila gene that encodes a potassium channel. Cell 50:405-413.

Kamb, A., Tseng-Crank, J. and Tanouye, M.A. 1988. Multiple products of the Drosophila Shaker gene may contribute to potassium channel diversity. Neuron 1:421-430.

Kashuba, V.I., Kvasha, S.M., Protopopov, A.I., Gizatullin, R.Z., Rynditch, A.V., Wahlestedt, C., Wasserman, W.W. and Zabarovsky, E.R. 2001. Initial isolation and analysis of the human Kv1.7 (KCNA7) gene, a member of the voltage-gated potassium channel gene family. Gene 268:115-122.

Kaufmann, E. and Knochel, W. 1996. Five years on the wings of fork head. Mech. Dev. 57:3-20.

Kennedy, M.C., Emptage, M.H. and Beinert, H. 1984. Incorporation of [35S]sulfide into the Fe-S cluster of aconitase. J. Biol. Chem. 259:3145-3151.

Kim, K.R., Moon, H.E. and Kim, K.W. 2002. Hypoxia-induced angiogenesis in human hepatocellular carcinoma. J. Mol. Med. 80:703-714.

Kiss, L. and Korn, S.J. 1998. Modulation of C-type inactivation by $\mathrm{K}^{+}$at the potassium channel selectivity filter. Biophys. J . 74:1840-1849.

Klein, P., Kanehisa, M. and DeLisi, C. 1985. The detection and classification of membrane-spanning proteins. Biochim. Biophys. Acta 815:468-476.

Klemic, K.G., Shieh, C.C., Kirsch, G.E. and Jones, S.W. 1998. Inactivation of Kv2.1 potassium channels. Biophys. J. 74:1779-1789.

Kozak, M. 1989. Circumstances and mechanisms of inhibition of translation by secondary structure in eucaryotic mRNAs. Mol. Cell. Biol. 9:5134-5142.

Kozak, M. 1989. The scanning model for translation: an update. J. Cell. Biol. 108:229-241.

Kozak, M. 1990. Downstream secondary structure facilitates recognition of initiator codons by eukaryotic ribosomes. Proc. Natl. Acad. Sci. USA 87:8301-8305.

Kozak, M. 1991. Structural features in eukaryotic mRNAs that modulate the initiation of translation. J. Biol. Chem. 266:19867-19870.

Kozak, M. 1999. Initiation of translation in prokaryotes and eukaryotes. Gene 234:187-208. 
Kramer, J.W., Post, M.A., Brown, A.M. and Kirsch, G.E. 1998. Modulation of potassium channel gating by coexpression of Kv2.1 with regulatory Kv5.1 or Kv6.1 alpha-subunits. Am. J. Physiol. 274:C1501-1510.

Krick, S., Platoshyn, O., Sweeney, M., McDaniel, S.S., Zhang, S., Rubin, L.J. and Yuan, J.X. 2002. Nitric oxide induces apoptosis by activating $\mathrm{K}^{+}$channels in pulmonary vascular smooth muscle cells. Am. J. Physiol. Heart Circ. Physiol. 282:H184-193.

Kuo, C.C. and Bean, B.P. 1994. $\mathrm{Na}^{+}$channels must deactivate to recover from inactivation. Neuron 12:819-829.

Kyte, J. and Doolittle, R.F. 1982. A simple method for displaying the hydropathic character of a protein. J. Mol. Biol. 157:105-132.

Labarca, P. and Mackinnon, R. 1992. Permeant ions influence the rate of C-type inactivation in Shaker channels. Biophys. J. 61:A378.

Lee, T.E., Philipson, L.H. and Nelson, D.J. 1996. N-type inactivation in the mammalian Shaker K+ channel Kv1.4. J. Membr. Biol. 151:225-35.

Levitan, E.S., Gealy, R., Trimmer, J.S. and Takimoto, K. 1995. Membrane depolarization inhibits $\mathrm{Kv} 1.5$ voltage-gated $\mathrm{K}^{+}$channel gene transcription and protein expression in pituitary cells. J. Biol. Chem. 270:6036-6041.

Levy, D.I. and Deutsch, C. 1996a. Recovery from C-type inactivation is modulated by extracellular potassium. Biophys. J. 70:798-805.

Levy, D.I. and Deutsch, C. 1996b. A voltage-dependent role for $\mathrm{K}^{+}$in recovery from C-type inactivation. Biophys. J. 71:3157-3166.

Liao, W., Hong, S.H., Chan, B.H., Rudolph, F.B., Clark, S.C. and Chan, L. 1999. APOBEC-2, a cardiac- and skeletal muscle-specific member of the cytidine deaminase supergene family. Biochem. Biophys. Res. Commun. 260:398-404.

Liman, E.R., Tytgat, J., Hess, P. 1992. Subunit stoichiometry of a mammalian K+ channel determined by construction of multimeric cDNAs. Neuron. 9:861-871.

Liu, Y., Jurman, M.E. and Yellen G. 1996. Dynamic rearrangement of the outer mouth of a K+ channel during gating. Neuron 16:859-867.

Loboda, A. and Armstrong, C.M. 2001. Resolving the gating charge movement associated with late transitions in $\mathrm{K}^{+}$channel activation. Biophys. J . 81:905-916.

Loots, E. and Isacoff, E.Y. 1998. Protein rearrangements underlying slow inactivation of the Shaker K+ channel. J. Gen. Physiol. 112:377-389.

Lopez-Barneo, J., Hoshi, T., Heinemann, S.H. and Aldrich, R.W. 1993. Effects of external cations and mutations in the pore region on C-type inactivation of Shaker potassium channels. Receptors Channels 1:61-71. 
Maas, S. and Rich, A. 2000. Changing genetic information through RNA editing. Bioessays 22:790-802.

MacDonald, P. E., Wang, G., Tsuk, S., Dodo, C., Kang, Y., Tang, L., Wheeler, M. B., Cattral, M. S., Lakey, J. R., Salapatek, A. M., Lotan, I. and Gaisano, H. Y. 2001. Members of the $\mathrm{Kv} 1$ and $\mathrm{Kv} 2$ voltage-dependent $\left.\mathrm{K}^{+}\right)$channel families regulate insulin secretion. Mol. Endocrinol. 15:1423-1435.

MacKinnon, R. and Miller, C. 1989. Mutant potassium channels with altered binding of charybdotoxin, a pore-blocking peptide inhibitor. Science 245:1382-1385.

MacKinnon, R. and Yellen, G. 1990. Mutations affecting TEA blockade and ion permeation in voltage-activated $\mathrm{K}^{+}$channels. Science 250:276-279.

MacKinnon, R. 1991. Determination of the subunits stoichiometry of a voltageactivated potassium channel. Nature 350:232-235.

MacKinnon, R., Aldrich, R. W. and Lee, A.W. 1993. Functional stoichiometry of Shaker potassium channel inactivation. Science 262:757-759.

McCormack, K., Joiner, W.J. and Heinemann, S.H. 1994. A characterization of the activating structural rearrangements in voltage-dependent Shaker $\mathrm{K}^{+}$channels. Neuron 12:301-315. Erratum in: Neuron 12:706.

Miller, C. 2001. See potassium run. Nature 414:23-24.

Miralles, F., Canti, C., Marsal, J., Peres, J., and Solsona, C. 1994. Zinc ions block rectifier potassium channels and calcium activated potassium channels at the frog motor nerve endings. Brain Res. 641(2):279-84.

Morais-Cabral, J.H., Zhou, Y. and MacKinnon, R. 2001. Energetic optimization of ion conduction rate by the $\mathrm{K}^{+}$selectivity filter. Nature 414:37-42.

Moran, O. 1996. Patch clamp technique. In: Experimental Techniques in Bioelectrochemistry. V. Brabec, D. Walz and G. Milazzo. Basel, Switzerland, Birkhauser Verlag.

Morii, E., Oboki, K., Kataoka, T.R., Igarashi, K. and Kitamura, Y. 2002. Interaction and cooperation of mi transcription factor (MITF) and myc-associated zinc-finger protein-related factor (MAZR) for transcription of mouse mast cell protease 6 gene. $J$. Biol. Chem. 277:8566-8571.

Movahed, P., Hogestatt, E.D. and Petersson, J. 2003. Effect of hypoxia on vasodilator responses to S-nitroso-N-acetylpenicillamine and levcromakalim in guinea pig basilar artery. Naunyn Schmiedebergs Arch. Pharmacol. 367:532-537.

Murrell-Lagnado, R.D. and Aldrich, R.W. 1993. Interactions of amino terminal domains of Shaker $\mathrm{K}$ channels with a pore blocking site studied with synthetic peptides. J. Gen. Physiol. 102:949-975. 
Murrell-Lagnado, R.D. and Aldrich, R.W. 1993. Energetics of Shaker K channels block by inactivation peptides. J. Gen. Physiol. 102:977-1003.

Nakai, K. and Kanehisa M. 1992. A knowledge base for predicting protein localization sites in eukaryotic cells. Genomics 14:897-911.

Nakai, K. and Horton, P. 1999. PSORT: a program for detecting the sorting signals of proteins and predicting their subcellular localization, Trends Biochem. Sci. 24:3435.

Nakamaru-Ogiso, E., Yano, T., Ohnish,i T. and Yagi, T. 2002. Characterization of the iron-sulfur cluster coordinated by a cysteine cluster motif (CXXCXXXCX27C) in the Nqo3 subunit in the proton-translocating NADH-quinone oxidoreductase (NDH-1) of Thermus thermophilus HB-8. J. Biol. Chem. 277:1680-1688.

Neher, E., Sakmann, B. and Steinbach, J.H. 1978. The extracellular patch clamp: a method for resolving currents through individual open channels in biological membranes. Pflügers Arch. 375:219-228.

Noda, M., S. Shimizu, T. Tanabe, T. Takai, T. Kayano, T.Ikeda, H. Takahashi, H. Nakayama, Y. Kanaoka, and Minamino, N. 1984. Primary Structure Of Electrophorus Electricus Sodium Channel Deduced From Cdna Sequence. Nature 312:121-127.

Olson, N.E. 1990. The MyoD family: a paradigm for development? Genes Dev. 4:1454-1461.

Osipenko, O.N., Tate, R.J. and Gurney, A.M. 2000. Potential role for Kv3.1b channels as oxygen sensors. Circ. Res. 86:534-540.

Patel, A.J., Lazdunski, M. and Honore, E. 1997. Kv2.1/Kv9.3, a novel ATPdependent delayed-rectifier $\mathrm{K}^{+}$channel in oxygen-sensitive pulmonary artery myocytes. EMBO J. 16:6615-6625.

Patton, D.E., Silva, T. and Bezanilla, F. 1997. RNA editing generates a diverse array of transcripts encoding squid $\mathrm{Kv} 2 \mathrm{~K}^{+}$channels with altered functional properties. Neuron 19:711-722.

Pelletier, J. and Sonenberg, N. 1985. Insertion mutagenesis to increase secondary structure within the $5^{\prime}$ noncoding region of a eukaryotic mRNA reduces translational efficiency. Cell 40:515-526.

Perez-Cornejo, P., Stampe, P. and Begenisich, T. 1998. Proton probing of the charybdotoxin binding site of Shaker K ${ }^{+}$channels. J. Gen. Physiol. 111:441-50.

Perez-García, M.T., Lopez-Lopez, J.R. and Gonzalez, C. 1999. Kvb1.2 subunit coexpression in HEK293 cells confers O2 sensitivity to Kv4.2 but not to Shaker channels. J. Gen. Physiol. 113:897-907.

Perozo, E., Cortes, D.M. and Cuello, L.G. 1998. Three-dimensional architecture and gating mechanism of a $\mathrm{K}^{+}$channel studied by EPR spectroscopy. Na.t Struct. Biol. 5:459-469. 
Polder, H. R., Planck, J., Weskamp, M., Ferber, M. and Terlau, H. 2003. An electronic device that measures series resistance during TEVC recording in Xenopus oocytes. Poster at 29 ${ }^{\text {th }}$ Göttingen Neurobiology Conference 2003.

Pongs, O., Kecskemethy, N., Muller, R., Krah-Jentgens, I., Baumann, A., Kiltz, H. H., Canal, I., Llamazares, S. and Ferrus, A. 1988. Shaker encodes a family of putative potassium channel proteins in the nervous system of Drosophila. EMBO J. 7:10871096.

Rasmusson, R.L., Zhang, Y., Campbell, D.L., Comer, M.B., Castellino, R.C., Liu, S., and Strauss, H.C. 1995. Bi-stable block by 4 -aminopyridine of a transient $\mathrm{K}^{+}$channel (Kv1.4) cloned from ferret ventricle and expressed in Xenopus oocytes. J. Physiol. 485:59-71.

Rasmusson, R.L., Morales, M.J., Castellino, R.C., Zhang, Y., Campbell, D.L. and Strauss, H.C. 1995. C-type inactivation controls recovery in a fast inactivation cardiac $\mathrm{K}^{+}$channel (Kv1.4) expressed in Xenopus oocytes. J. Physiol. 489:709-721.

Rasmusson, R.L., Morales, M.J., Wang, S., Liu, S., Campbell, D.L., Brahmajothi, M.V. and Strauss, H.C. 1998. Inactivation of voltage-gated cardiac $\mathrm{K}^{+}$channels. Circ Res 82:739-750.

Rettig, J., Heinemann, S.H., Wunder, F., Lorra, C., Parcej, D.N., Dolly, J.O. and Pongs, O. 1994. Inactivation properties of voltage-gated $\mathrm{K}^{+}$channels altered by presence of beta-subunit. Nature 369:289-294.

Ruppersberg, J.P., Schroter, K.H., Sakmann, B., Stocker, M., Sewing, S. and Pongs, O. 1990. Heteromultimeric channels formed by rat brain potassium channel proteins. Nature 345:535-537.

Ruppersberg, J.P., Stocker, M., Pongs, O., Heinemann, S.H., Frank, R. and Koenen M. 1991. Regulation of fast inactivation of cloned mammalian IK(A) channels by cysteine oxidation. Nature 352:711-714.

Rouault, T.A., Haile, D.J., Downey, W.E., Philpott, C.C., Tang, C., Samaniego, F., Chin, J., Paul, I., Orloff, D. and Harford, J.B 1992. An iron-sulfur cluster plays a novel regulatory role in the iron-responsive element binding protein. Biometals 5:131-140.

Rouillard, J.M., Zuker, M. and Gulari, E. 2003. OligoArray 2.0: design of oligonucleotide probes for DNA microarrays using a thermodynamic approach. Nucleic Acids Res. 31:3057-3062.

Saiki, R.K., Gelfand, D.H., Stoffel, S., Scharf, S.J., Higuchi, R., Horn, G.T., Mullis, K.B. and Erlich, H.A. 1988. Primer-directed enzymatic amplification of DNA with a thermostable DNA polymerase. Science 239:487-91.

Salvaterra, C.G. and Goldman, W.F. 1993. Acute hypoxia increases cytosolic calcium in cultured pulmonary arterial myocytes. Am. J. Physiol. 264 :323-328. 
Sambrook, J., Fritsch, E.F. and Maniatis, T. 1989. Molecular Cloning. A Laboratory Manual. Cold Spring Harbor Laboratory Press, $2^{\text {nd }}$ Edition.

Schwarz, T.L., Tempel, B.L., Papazian, D.M., Jan, Y.N., and Jan, L.Y. 1988. Multiple potassium-channel components are produced by alternative splicing at the Shaker locus in Drosophila. Nature 331(6152):137-42. Erratum in: Nature 332:740.

Seeburg, P.H., Higuchi M. and Sprengel, R. 1998. RNA editing of brain glutamate receptor channels: mechanism and physiology. Brain Res. Rev. 1998, 26(2-3):21729. Review.

Shieh, C.C., Klemic, K.G. and Kirsch, G.E. 1997. Role of transmembrane segment S5 on gating of voltage-dependent K ${ }^{+}$channels. J. Gen. Physiol. 109(6):767-778.

Sigworth, F.J. 1994. Voltage gating of ion channels. Q. Rev. Biophys. 27:1-40.

Smith-Maxwell, C.J., Ledwell, J.L. and Aldrich, R.W. 1998. Uncharged S4 residues and cooperativity in voltage-dependent potassium channel activation. J. Gen. Physiol. 111:421-439.

Starace, D.M. and Bezanilla, F. 2004. A proton pore in a potassium channel voltage sensor reveals a focused electric field. Nature 427:548-553

Sweeney, M. and Yuan, J.X. 2000. Hypoxic pulmonary vasoconstriction: role of voltage-gated potassium channels. Respir. Res. 1:40-48.

Swenson, R.P. Jr. and Armstrong, C.M. 1981. $\mathrm{K}^{+}$channels close more slowly in the presence of external $\mathrm{K}^{+}$and $\mathrm{Rb}^{+}$. Nature 291:427-429.

Szabo, S.J., Glimcher, L.H. and Ho, I.C. 1997. Genes that regulate interleukin-4 expression in T cells. Curr Opin. Immunol. 9:776-781.

Tang, W. and Tseng, H. 1999. A GC-rich sequence within the 5' untranslated region of human basonuclin mRNA inhibits its translation. Gene 237:35-44.

Tempel, B., Papazian, D., Schwarz, T., Jan, Y. and Jan, L. 1987. Sequence of a probable potassium channel component encoded at Shaker locus of Drosophila. Science 237:770-775.

Terlau, H., Heinemann, S.H., Stühmer, W., Pongs, O. and Ludwig, J. 1997. Amino terminal-dependent gating of the potassium channel rat eag is compensated by a mutation in the S4 segment. J. Physiol. 502:537-543.

Terlau, H., Boccaccio, A., Olivera, B.M. and Conti F. 1999. The block of Shaker K+ channels by kappa-conotoxin PVIIA is state dependent. J. Gen. Physiol. 114:125140.

Thompson, J. and Begenisich, T. 2003. External TEA block of shaker $\mathrm{K}^{+}$channels is coupled to the movement of $\mathrm{K}^{+}$ions within the selectivity filter. J. Gen. Physiol. 122:239-246. 
Vallee, B.L. and Auld D.S. 1990. Zinc coordination, function, and structure of zinc enzymes and other proteins. Biochemistry 29:5647-5659.

VanDongen, A.M., Frech, G.C., Drewe, J.A., Joho, R.H. and Brown, A.M. 1990. Alteration and restoration of $\mathrm{K}^{+}$channel function by deletions at the $\mathrm{N}$ - and $\mathrm{C}$-termini. Neuron 5:433-443.

Vega-Saenz de Miera, E. and Rudy, B. 1992. Modulation of $\mathrm{K}^{+}$channels by hydrogen peroxide. Biochem. Biophys. Res. Commun. 186:1681-1687.

Wang, H., Kunkel, D.D., Martin, T.M., Schwartzkroin, P.A. and Tempel, B.L. 1993. Heteromultimeric potassium channels in the terminal and juxtaparanodal regions of neurons. Nature 365:75-79.

Wang, G.L., Jiang, B.H. and Semenza, G.L. 1995. Effect of altered redox states on expression and DNA-binding activity of hypoxia-inducible factor 1. Biochem. Biophys. Res. Commun. 212:550-556.

Wang, G.L. and Semenza, G.L. 1995. Purification and characterization of hypoxiainducible factor 1. J. Biol. Chem. 270:1230-1237.

Wang, Y., Shen, J., Arenzana, N., Tirasophon, W., Kaufman, R.J. and Prywes, R. 2000. Activation of ATF6 and an ATF6 DNA binding site by the endoplasmic reticulum stress response. J. Biol. Chem. 275:27013-27020.

White, M.M. and Bezanilla, F. 1985. Activation of squid axon $\mathrm{K}^{+}$channels. Ionic and gating current studies. J. Gen. Physiol. 85:839-554.

Wissmann, R., Baukrowitz, T., Kalbacher, H., Kalbitzer, H.R., Ruppersberg, J.P., Pongs, O., Antz, C. and Fakler, B. 1999. NMR structure and functional characteristics of the hydrophilic $\mathrm{N}$ terminus of the potassium channel beta-subunit Kvbeta1.1. J. Biol. Chem. 274:35521-35525.

Wormald, M.R. and Dwek, R.A. 1999. Glycoproteins: glycan presentation and protein-fold stability. Structure Fold Des. 7:R155-156.

Yellen, G., Sodickson, D., Chen,T.Y. and Jurman, M.E. 1994. An engineered cysteine in the external mouth of a $\mathrm{K}^{+}$channel allows inactivation to be modulated by metal binding. Biophys. J. 66:1068-1075.

Yellen, G. 1998. The moving parts of voltage-gated ion channels. Q. Rev. Biophys. 31:239-295.

Zagotta, W.N. and Aldrich, R.W. 1990. Voltage-dependent gating of Shaker A-type potassium channel in Drosophila muscle. J. Gen. Physiol. 95:29-60.

Zhou, Y., Morais-Cabral, J.H., Kaufman, A. and MacKinnon, R. 2001. Chemistry of ion coordination and hydration revealed by a $\mathrm{K}^{+}$channel-Fab complex at $2.0 \mathrm{~A}$ resolution. Nature 414:43-48. 
Zollman, S., Godt, D., Prive, G.G., Couderc, J.L. and Laski, F.A. 1994. The BTB domain, found primarily in zinc finger proteins, defines an evolutionarily conserved family that includes several developmentally regulated genes in Drosophila. Proc. Natl. Acad. Sci. USA 91:10717-10721. 


\section{Lebenslauf}

Name:

Geburtsdatum:

Geburtsort:

Nationalität:

Adresse:

\section{Bildungsweg}

$1977-1990$

$1990-1991$

$1992-1999$

$1994-1996$

$1995-1997$

$1995-1999$

seit 2000
Rocio Karin Finol-Urdaneta

17. Dezember 1971

Maracaibo, Venezuela

venezuelanisch

Theodor-Heuss-Str. 35

37075 Göttingen

Tel. 0551-3898 170
Grundschule und Gymnasium "Colegio San Vicente de Paul", Maracaibo, Venezuela mit Abschluss "Abitur"

Computerfachausbildung am "Colegio Universitario Rafael Belloso Chacin", Maracaibo, Venezuela

Studium der Biologie an der "La Universidad del Zulia", Maracaibo, Venezuela mit Abschluss "Diplom"

Wissenschaftliche Hilfskraft an der "La Universidad del Zulia" im Labor für Physiologie der Mikroorganismen, Maracaibo, Venezuela

Wissenschaftliche Hilfskraft an der "La Universidad del Zulia”, im Labor für Ökologie der Mikroorganismen und Biotechnologie, Maracaibo, Venezuela

Wissenschaftliche Hilfskraft an der "La Universidad del Zulia", im Institut für Klinische Forschung, Abt. Biochemie, Maracaibo, Venezuela

Dissertation im Max-Planck-Institut für Experimentelle Medizin, Göttingen, mit dem Thema "Investigation of the heterologous expression of the voltage activated potassium channel Kv1.7" bei PD Dr. Heinrich Terlau 